Portland State University

PDXScholar

Summer 7-6-2016

\title{
Wake Character in the Wind Turbine Array: (Dis- )Organization, Spatial and Dynamic Evolution and Low-dimensional Modeling
}

Nicholas Michael Hamilton

Portland State University

Follow this and additional works at: https://pdxscholar.library.pdx.edu/open_access_etds

Part of the Energy Systems Commons, and the Power and Energy Commons Let us know how access to this document benefits you.

\section{Recommended Citation}

Hamilton, Nicholas Michael, "Wake Character in the Wind Turbine Array: (Dis-)Organization, Spatial and Dynamic Evolution and Low-dimensional Modeling" (2016). Dissertations and Theses. Paper 3084. https://doi.org/10.15760/etd.3079

This Dissertation is brought to you for free and open access. It has been accepted for inclusion in Dissertations and Theses by an authorized administrator of PDXScholar. Please contact us if we can make this document more accessible: pdxscholar@pdx.edu. 
Wake Character in the Wind Turbine Array: (Dis-)Organization, Spatial and Dynamic Evolution and Low-dimensional Modeling

by

Nicholas Michael Hamilton

A dissertation submitted in partial fulfillment of the requirements for the degree of

\author{
Doctor of Philosophy \\ in \\ Mechanical Engineering
}
Dissertation Committee:
Raúl Bayoán Cal, Chair
Murat Tutkun
Mark Weislogel
Stefan Talke

Portland State University

2016 


\begin{abstract}
To maximize the effectiveness of the rapidly increasing capacity of installed wind energy resources, new models must be developed that are capable of more nuanced control of each wind turbine so that each device is more responsive to inflow events. Models used to plan wind turbine arrays and control behavior of devices within the farm currently make questionable estimates of the incoming atmospheric flow and update turbine configurations infrequently. As a result, wind turbines often operate at diminished capacities, especially in arrays where wind turbine wakes interact and inflow conditions are far from ideal. New turbine control and wake prediction models must be developed to tune individual devices and make accurate power predictions. To that end, wind tunnel experiments are conducted detailing the turbulent flow in the wake of a wind turbine in a model-scale array. The proper orthogonal decomposition (POD) is applied to characterize the spatial evolution of structures in the wake. Mode bases from distinct downstream locations are reconciled through a secondary decomposition, called double proper orthogonal decomposition (DPOD), indicating that modes of common rank in the wake share an ordered set of sub-modal projections whose organization delineates underlying wake structures and spatial evolution. The doubly truncated basis of sub-modal structures represents a reduction to $0.015 \%$ of the total degrees of freedom of the wind turbine wake. Low-order representations of the Reynolds stress tensor are made using only the most dominant DPOD modes, corrected to account for energy excluded from the truncated basis with a tensor of constant coefficients defined to rescale the low-order representation of the stresses
\end{abstract}


to match the original statistics. Data from the wind turbine wake are contrasted against simulation data from a fully-developed channel flow, illuminating a range of anisotropic states of turbulence. Complexity of flow descriptions resulting from truncated POD bases is suppressed in severe basis truncations, exaggerating anisotropy of the modeled flow and, in extreme cases, can lead to the loss of three dimensionality. Constant corrections to the low-order descriptions of the Reynolds stress tensor reduce the root-mean-square error between low-order descriptions of the flow and the full statistics as much as $40 \%$ and, in some cases, reintroduce three-dimensionality to severe truncations of POD bases. Low-dimensional models are constructed by coupling the evolution of the dynamic mode coefficients through their respective time derivatives and successfully account for non-linear mode interaction. Deviation between time derivatives of mode coefficients and their least-squares fit is amplified in numerical integration of the system, leading to unstable long-time solutions. Periodic recalibration of the dynamical system is undertaken by limiting the integration time and using a virtual sensor upstream of the wind turbine actuator disk in to read the effective inflow velocity. A series of open-loop transfer functions are designed to inform the low-order dynamical system of the flow incident to the wind turbine rotor. Validation data shows that the model tuned to the inflow reproduces dynamic mode coefficients with little to no error given a sufficiently small interval between instances of recalibration. The reduced-order model makes accurate predictions of the wake when informed of turbulent inflow events. The modeling scheme represents a viable path for continuous time feedback and control that may be used to selectively tune a wind turbine in the effort to maximize power output of large wind farms. 


\section{Acknowledgements}

In the course of my doctoral studies, I have benefited from incredible support from friends and family, colleagues and institutions. I must first thank the tireless persistence of my advisors and mentors Raúl Bayoán Cal and Murat Tutkun, who have spent countless hours helping me to develop ideas and guiding me forward. They have seen all of my work, often many times and in many incarnations as projects coalesce and evolve. They have been instrumental in keeping me focused on the tasks at hand, and introducing me to new concepts and tools necessary for work herein. My colleagues in the PhD program deserve special thanks, as well. Matt Melius, Drew Wollman, Naseem Ali, and Elizabeth Camp all have a part in this work, through offering insight and inspiration from their own studies and experiences. Gerard Cortina and Marc Calaf stepped in to provide me with eleventh-hour simulation data when I realized that what I needed was out of reach in my own data. My family and loved ones have offered their unwavering dedication to my many years of study, as well. They are excited to hear what I'm working on, and excited to ask me to explain again using coherent thoughts (and complete sentences). Thank you, Leela and Riley. I cannot express how important it is to have you with me, in years past and in those to come. 


\section{Table of Contents}

Abstract

Acknowledgements

$\begin{array}{ll}\text { List of Tables } & \text { v }\end{array}$

List of Figures

$\begin{array}{lll}\text { Chapter } 1 & \text { Introduction } & 1\end{array}$

$\begin{array}{lll}\text { Chapter } 2 & \text { Theory } & 15\end{array}$

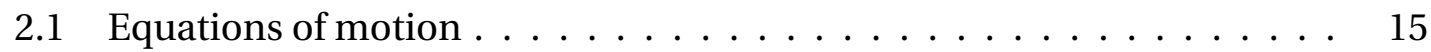

2.2 Phase dependent statistics . . . . . . . . . . . . . 17

2.3 Anisotropy of the turbulent stress tensor . . . . . . . . . . . . . . . 19

2.4 Snapshot proper orthogonal decomposition $\ldots \ldots \ldots 22$

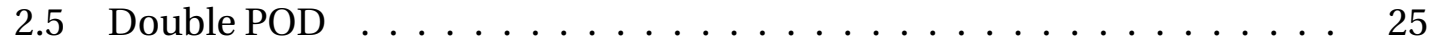

2.6 Galerkin Projection $\ldots \ldots \ldots \ldots \ldots \ldots$

2.7 Least squares polynomial fit $\ldots \ldots \ldots \ldots \ldots \ldots \ldots$

$\begin{array}{lll}\text { Chapter } 3 & \text { Experimental setup } & 37\end{array}$

3.1 Wind tunnel Experiment $\ldots \ldots \ldots \ldots \ldots \ldots$ 
3.2 Turbulent channel flow - direct numerical simulation . . . . . . . . . 41

3.3 Large eddy simulations of wind farms $\ldots \ldots \ldots \ldots$. . . . . . . 43

$\begin{array}{lll}\text { Chapter } 4 & \text { Results } & 48\end{array}$

4.1 Phase-locked wind turbine wake statistics $\ldots \ldots \ldots \ldots$

4.1 .1 Conclusions ......................... 59

4.2 Double proper orthogonal decomposition of a wind turbine wake . . . 63

4.2.1 Wake flow characterization .............. 63

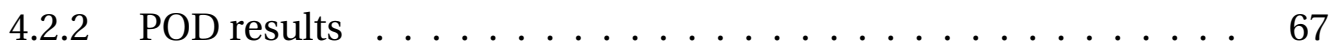

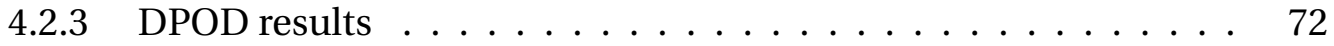

4.2.4 Corrected low-order description . . . . . . . . . . . 81

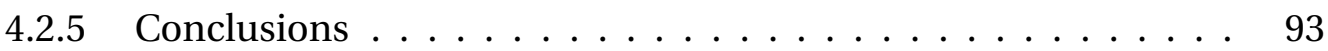

4.3 Turbulence anisotropy and the proper orthogonal decomposition _ . . 98

4.3 .1 Turbulence field . . . . . . . . . . . . . . . . . 98

4.3.2 Reynolds stress anisotropy . . . . . . . . . . . . . . . . . 104

4.3.3 Snapshot POD results . . . . . . . . . . . . . . 107

4.3.4 Error propagation via basis truncation . . . . . . . . . . . 117

4.3.5 Correction of the reduced order flow description . . . . . . . . 122

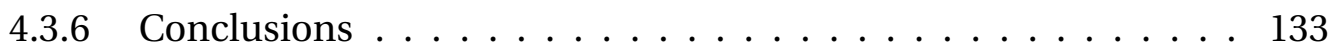

4.4 Dynamical systems of a fully developed turbulent channel flow . . . . . 137

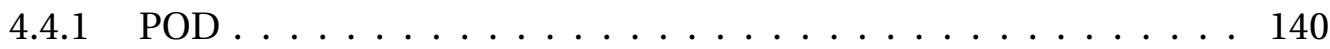

4.4 .2 Least-squares polynomial fit . . . . . . . . . . . . 143

4.4.3 Periodic LODS recalibration $\ldots \ldots \ldots$. . . . . . . . . 152

4.4 .4 Conclusions ........................ 160

4.5 Reduced-order model of a wind turbine wake . . . . . . . . . . . . . . . 164 
4.5 .1 Conclusions . . . . . . . . . . . . . . . 187

4.6 Reduced-order wind turbine array model . . . . . . . . . . . . . . 190

4.6 .1 Conclusion . . . . . . . . . . . . . . . . 199

$\begin{array}{lll}\text { Chapter } 5 & \text { Conclusions } & 203\end{array}$

5.1 Review of findings . . . . . . . . . . . . . . . . . 203

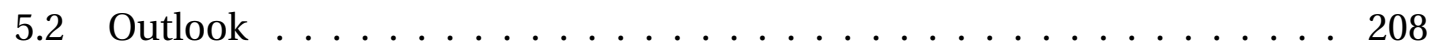

$\begin{array}{ll}\text { Bibliography } & 213\end{array}$

Appendix A Anisotropy of severely truncated bases 222

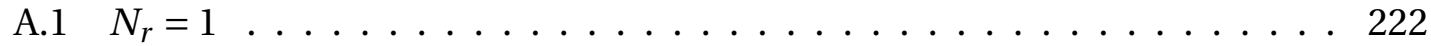

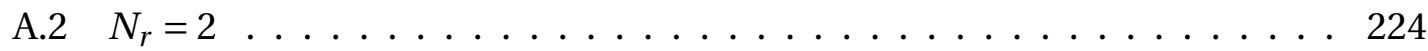

Appendix B Galerkin projection of channel flow plane data 227

Appendix C DPOD LODS

C.1 Least squares polynomial fit . . . . . . . . . . . . . 236

C.2 reconstructions and error calcs . . . . . . . . . . . . 240

Appendix D Turbulent channel flow documentation $\quad 248$

$\begin{array}{ll}\text { Appendix E Open-loop transfer functions } & 257\end{array}$ 


\section{List of Tables}

Table 2.1 Limiting cases of turbulence given on the Lumley triangle in terms of anisotropy tensor invariants. . . . . . . . . . 21

Table 3.1 Details of sampled volume data. . . . . . . . . . . . . . 43

Table 4.1 Normalized RMS error difference $d a_{i} / d t$ and $d \hat{a}_{i} / d t$ according to the number modes $N_{r}$ used in the LODS. . . . . . . . . . . 151

Table 4.2 Normalized RMS difference between $a_{i}$ and $\hat{a}_{i}$ according to the number modes $N_{r}$ used in the LODS. . . . . . . . . . . . . . 152

Table 4.3 Span error (\%) between $\overline{u_{i} u_{j}}$ between the sampled turbulence field and approximations from the POD or the LODS with periodic recalibration. . . . . . . . . . . . . . . . . . 159

Table 4.4 Number of poles, zeros, and the goodness of fit for transfer functions relating $U_{\text {eff }}$ to each mode coefficient. . . . . . . . . . . 179

Table 4.5 Comparison of goodness of fit values with and without application of constant correction. . . . . . . . . . . . . 187

Table 4.6 Details for wake IV transfer functions including number of poles, zeros, and the goodness of fit relating $U_{\mathrm{eff}, 4}$ to each mode coeffi-

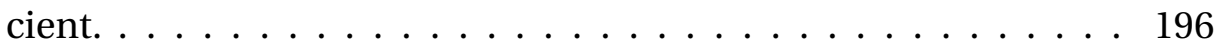


Table 4.7 Transfer function parameters for wake V. The first set of poles, zeros, and fit quality refer to the LES inflow signal. The second set refer to the LODS model from wake IV. . . . . . . . . . . . . . . . 199 


\section{List of Figures}

Figure 2.1 Lumley triangle showing limits of realizable turbulence according to the anisotropy tensor invariants $\eta$ and $\xi$. . . . . . . . . 2

Figure 2.2 Conceptual diagram showing the analysis procedure from velocity snapshots through applications of the POD and formation of a low-order description of the turbulent wind turbine wake. . . .

Figure 3.1 Schematic of the model-scale wind turbines used in the wind turbine array, figure 3.1(A). Model is also shown relative to the SPIV measurement window in the wakes with affixed coordinate system, figure $3.1(\mathrm{~B}) \ldots \ldots \ldots \ldots \ldots$

Figure 3.2 Model-scale array used in the current experiment relating turbine spacing and measurement locations behind the fourth row.

Figure 3.3 Schematic of the lower half of the channel flow DNS simulation space. Only a small region of the total channel is shown. Sampling window (white rectangle) was sized to span the inner layers. Location of the window in $x / H$ and $z / H$ was selected ran-

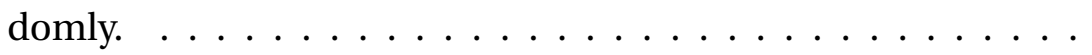

Figure 3.4 Half-channel velocity profile. Dashed lines correspond to the viscous sublayer and the log-layer. . . . . . . . . . . . . 
Figure 3.5 Simulation domain for wind turbine LES. Inner volume represents the subdomain analyzed in the present work. The light gray circle indicates the swept area of the rotor over which velocity is integrated to calculate $U_{\text {eff. }} \ldots \ldots \ldots \ldots$

Figure 4.1 Time averaged streamwise velocity, $U / U_{h u b}$. The contours above show the mean velocity averaged over all measurements and are independent of phase. . . . . . . . . . . . . . 49

Figure 4.2 Time averaged Reynolds stresses; independent of phase. . . . . . 50

Figure $4.3 \quad$ Phase-averaged Reynolds stresses in the near wake. . . . . . . . 51

Figure 4.4 Phase-averaged flux of kinetic energy $\langle u v U\rangle_{\alpha} \ldots \ldots \ldots$

Figure 4.5 Normal and shear deterministic stresses composed of ensemble averaged deviations between phase-averaged and total mean velocities at $x / D=0.5 \ldots \ldots \ldots \ldots \ldots \ldots$

Figure 4.6 Contours representing the contribution of the total flux by the streamwise-wall-normal deterministic stress $-\overline{u^{\prime \prime} v^{\prime \prime}} U$. . . . .

Figure 4.7 Tertiary stresses in the near wake. Shown in the figure are the streamwise contribution, analogous to deterministic turbulence intensity and the components that contribute to kinetic energy

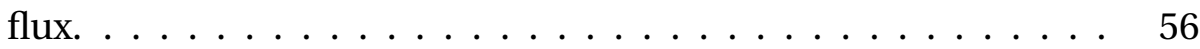

Figure 4.8 The entrainment process described in Cartesian and polarcylindrical coordinate systems. . . . . . . . . . . . . 58

Figure 4.9 Contours representing the radial flux of mean kinetic energy into the wake of a wind turbine, $F_{x r}=\overline{u_{x} u_{r}} U_{x} \ldots \ldots \ldots 59$

Figure 4.10 Flow statistics for wind turbine wake. Flow is from left to right. . 64 
Figure 4.11 Eigenvalues of the POD $\left(\lambda^{(n)}\right)$ according to streamwise coordinate, figure 4.33(A). The normalized cumulative summation of eigenvalues is shown in figure 4.11(B). Only the first 100 eigenvalues are included. . . . . . . . . . . . . . . 67

Figure 4.12 Cumulative fraction of $\lambda$ according to streamwise coordinate. The number of modes required to reach the listed thresholds are nearly constant in the wake. . . . . . . . . . . . . . 68

Figure 4.13 POD mode 1 in the wake. From top are the $\phi_{u}, \phi_{v}$, and $\phi_{w}$ com-

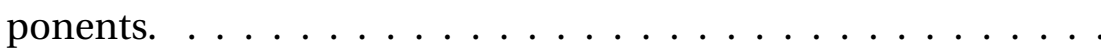

Figure 4.14 POD mode 2 in the wake. From top are the $\phi_{u}, \phi_{v}$, and $\phi_{w}$ com-

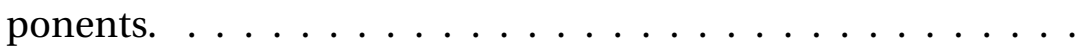

Figure 4.15 The 100th POD mode, $\boldsymbol{\Phi}^{(100)}$. Spatial organization into large structures is not evident in any but low mode numbers. From top are the $\phi_{u}, \phi_{v}$, and $\phi_{w}$ components. . . . . . . . . 72

Figure 4.16 Reconstructions of turbulence stresses based on 50\% energy delineated by the cumulative fraction of $\lambda \ldots \ldots \ldots 73$

Figure 4.17 Distribution of eigenvalues associated with each sub-modal structure in wind turbine wake. . . . . . . . . . . . . 75

Figure 4.18 Span of the first set of DPOD modes, $\Gamma^{(1, m)}$. From top are the $\gamma_{u}$, $\gamma_{v}$, and $\gamma_{w}$ components. . . . . . . . . . . . 77

Figure 4.19 Span of the second set of DPOD modes, $\Gamma^{(2, m)}$. From top are the $\gamma_{u}, \gamma_{v}$, and $\gamma_{w}$ components. $\ldots \ldots \ldots \ldots \ldots$

Figure 4.20 DPOD of intermediate POD mode, $\Gamma^{(100, m)}$. From top are the $\gamma_{u}$, $\gamma_{v}$, and $\gamma_{w}$ components. $\ldots \ldots \ldots \ldots \ldots \ldots$ 
Figure 4.21 Sub-modal amplitudes $b_{n, m}$ associated with (a) $\boldsymbol{\Gamma}^{(1, m)}$, (b) $\boldsymbol{\Gamma}^{(2, m)}$,

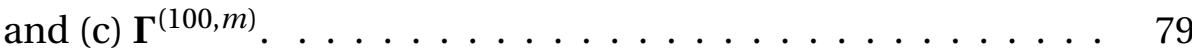

Figure 4.22 Comparisons of the shear stress $-\overline{u v}$ based on varying levels of sub-modal filtering. From left are the original statistical stress, the POD reduced order description, and the stress filtered with 1,2 , and 3 of the 13 sub-modes. . . . . . . . . .

Figure 4.23 Convergence of $\frac{\circ}{u u}$ and $-\frac{\circ}{u v}$ to original statistical profiles using progressively more modes in reconstruction. Low-order descriptions with a single POD mode are shown as solid dark gray lines; descriptions with two POD modes dark gray dashed lines. Higher order descriptions are shown in light gray lines and the original statistics are shown in black. Profiles correspond to the data at $x / D=1.5$, where the highest magnitudes of Reynolds stresses occur. . . . . . . . . . . . . .

Figure 4.24 Coefficients (left column) and error (right column) associated with correction of low-order descriptions of the streamwise normal stress $(\stackrel{\circ}{u u})$ and shear stresses responsible for entrainment $\left(-\frac{\circ}{u v}\right.$ and $\left.-\frac{\circ}{u w}\right)$ as a function of number of modes used in loworder representation of stresses $(n)$ and streamwise coordinate $(x / D) \ldots \ldots \ldots \ldots \ldots \ldots \ldots \ldots \ldots \ldots \ldots \ldots \ldots \ldots \ldots \ldots \ldots$

Figure 4.25 Correction coefficient and associate error for severe reduction (two modes, $\left.N_{r}=2\right) \ldots \ldots \ldots \ldots \ldots \ldots$ 
Figure 4.26 Comparison of $\overline{u u}$ to $\left.C_{11} \frac{\circ}{u u}\right|_{2} ^{2}$. The modeled normal stress diverges from the statistics most following the mast of the model wind turbine at $z / D=0 \ldots \ldots \ldots \ldots$. . . . . . . . 91

Figure 4.27 Comparison of $-\overline{u v}$ to $-\left.C_{12} \frac{\circ}{u v}\right|_{2} ^{2}$. Error associated with model correction is largely due to the overestimation of $-\overline{u v}$ in the near

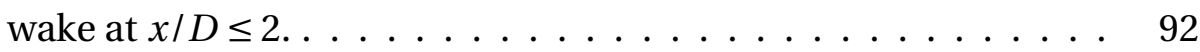

Figure 4.28 Comparison of $-\overline{u w}$ to $-\left.C_{12} \frac{\circ}{u w}\right|_{2} ^{2}$. Features in the near wake $(x / D=0.5)$ are simplified in the low-order description. $\ldots \ldots$

Figure 4.29 Reynolds stresses tensor and $k$ from the wake of a wind turbine at $x / D=0.5 \ldots \ldots \ldots \ldots \ldots \ldots \ldots \ldots \ldots \ldots \ldots \ldots \ldots \ldots \ldots$

Figure 4.30 Reynolds stresses tensor and $k$ from the wake of a wind turbine at $x / D=6 \ldots \ldots \ldots \ldots \ldots \ldots \ldots \ldots \ldots$

Figure 4.31 Reynolds stresses tensor and $k$ from the fully developed channel flow DNS. . . . . . . . . . . . . . . . . 103

Figure 4.32 Anisotropy tensor invariants and corresponding Lumley triangles for included data. . . . . . . . . . . . . . . 106

Figure 4.33 Eigenvalues from the snapshot POD for the wind turbine wake at $x / D=0.5$ (solid black lines), $x / D=6$ (dashed lines), and the channel flow DNS data (circles). . . . . . . . . . . . . . . . . . 109

Figure 4.34 Anisotropic (top) and isotropic (bottom) contributions to selected turbulence statistics. At $x / D=0.5,50 \%$ energy requires

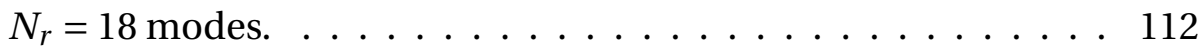


Figure 4.35 Anisotropic (top) and isotropic (bottom) contributions to selected turbulence statistics. At $x / D=6,50 \%$ energy requires $N_{r}=13$ modes. . . . . . . . . . . . . . . . 113

Figure 4.36 Anisotropic (top) and isotropic (bottom) contributions to selected turbulence statistics. For the channel flow, 50\% energy requires $N_{r}=8$ modes. . . . . . . . . . . . . . . . 114

Figure 4.37 Lumley triangle composed with invariants derived from $\overline{u_{i} u_{j}}$ (left), $\frac{\circ}{u_{i} u_{j}}$ (center), and $\hat{u_{i} u_{j}}$ (right). In each case, the $50 \%$ threshold on $N_{r}$ is used. . . . . . . . . . . . . . . . 115

Figure 4.38 RMS error associated with reconstructed Reynolds stress tensor as a function of number of basis modes. In all subfigures, $\overline{u u}$ solid black lines, $\overline{v v}$ dashed lines, $\overline{w w}$ dash-dot lines, $-\overline{u v}$ circles, $-\overline{u w}$ diamonds, $-\overline{v w}$ squares. . . . . . . . . . . . . . . 118

Figure 4.39 RMS error of anisotropy tensor invariants as a function of number of basis modes $\left(N_{r}\right)$. Mode numbers are normalized by the total number of modes, $N$. Lines are $x / D=0.5$ (solid black lines), $x / D=6$ (dashed lines), and the channel flow DNS data (circles). 119

Figure 4.40 Lumley triangle for channel flow represented with increasing number of POD modes. 4.40(A) $N_{r}=1$ (all data fall on a single point where $\eta=\xi=1 / 3$ ), 4.40(B) $N_{r}=2,4.40$ (C) $N_{r}=4,4.40$ (D) $N_{r}=8,4.40(\mathrm{E}) N_{r}=16,4.40(\mathrm{~F}) N_{r}=32,4.40(\mathrm{G})$ full data for reference. ....................... 120 
Figure 4.41 Correction coefficient $C_{i j}$ as a function of $N_{r}$. In all subfigures, $\overline{u u}$ solid black lines, $\overline{v v}$ dashed lines, $\overline{w w}$ dash-dot lines, $-\overline{u v}$ circles, $-\overline{u w}$ diamonds, $-\overline{v w}$ squares. . . . . . . . . . . . . . . 123

Figure 4.42 RMS error between $\overline{u_{i} u_{j}}$ and $C_{i j} \frac{\circ}{\overline{u_{i}} u_{j}}$. In all subfigures, $\overline{u u}$ solid black lines, $\overline{v v}$ dashed lines, $\overline{w w}$ dash-dot lines, $-\overline{u v}$ circles, $-\overline{u w}$ diamonds, $-\overline{v w}$ squares. . . . . . . . . . . . . . . . . 124

Figure 4.43 Lumley triangle for channel flow represented with increasing number of POD modes and correction factor $C_{i j} \cdot 4.43(\mathrm{~A}) N_{r}=1$, 4.43(B) $N_{r}=2,4.43(\mathrm{C}) N_{r}=4,4.43(\mathrm{D}) N_{r}=8,4.43$ (E) $N_{r}=16$, 4.43(F) $N_{r}=32,4.43(\mathrm{G})$ full data for reference. . . . . . . . . 127

Figure 4.44 RMS error between anisotropy invariants derived from corrected stress tensor and statistical values. . . . . . . . . . . . . . 128

Figure 4.45 Correction to selected stresses using the ratio of isotropic to anisotropic contributions. Due to extreme values, the logarithm of the correction tensor $\log \left(D_{i j}\right)$ is shown. . . . . . . . . 130

Figure 4.46 Lumley triangles with anisotropy invariants derived from decomposition of $D_{i j} \frac{o}{u_{i} u_{j}} \ldots \ldots \ldots \ldots \ldots \ldots \ldots$

Figure 4.47 Streamwise, wall-normal, and spanwise mean velocities in channel flow scaled by the bulk velocity. . . . . . . . . . . . 138

Figure 4.48 Turbulence kinetic energy and selected Reynolds shear stresses for sampled channel flow volume. . . . . . . . . . . . . . . . 139

Figure 4.49 Normalized POD eigenvalues (a) and their cumulative summation (b) along with energy thresholds. 5-modes are needed to reach the $50 \%$ energy threshold. . . . . . . . . . . . . . 140 
Figure 4.50 POD modes from channel flow volume. From left are modes 1 through $5 . \ldots \ldots \ldots \ldots \ldots \ldots \ldots \ldots$

Figure 4.51 Constant 4.51(A), linear 4.51(B), and quadratic 4.51(C) parameters from least-squares fit of $d \hat{a}_{i} / d t$. For the quadratic term shown, the index $k=1$ is fixed and other components are omitted. Cubic term is not shown for brevity. . . . . . . . . . . . . 145

Figure 4.52 Time derivatives of POD mode coefficients (black) and their respective LODS approximations (blue). . . . . . . . . . . . 147

Figure 4.53 Time derivatives of POD mode coefficients (black) and their respective LODS approximations (blue). . . . . . . . . . . . 149

Figure 4.54 POD mode coefficients (black) and their respective LODS approximations (blue) calculated from the time derivatives shown in Figure $4.53 . \ldots \ldots \ldots \ldots \ldots$

Figure 4.55 RMS difference of mode coefficients and their time derivatives (normalized by span) between the POD and the LODS. Error shown corresponds with the $N_{r}=14$ rows of tables 4.1 and $4.2 . \quad 152$

Figure 4.56 Histograms depicting 4.56(A) mode selected in periodic recalibration and 4.56(B) index selected by matching coefficients. . . 153

Figure 4.57 POD mode coefficients (black) and their respective LODS approximations (blue) calculated from the time derivatives shown in Figure $4.53 . \ldots \ldots \ldots \ldots \ldots$

Figure 4.58 Comparison of streamwise normal Reynolds stress from sample statistics, POD reconstruction, and LODS estimate, from left. . . 156 
Figure 4.59 Comparison of streamwise/wall-normal Reynolds shear stress from sample statistics, POD reconstruction, and LODS estimate, from left. . . . . . . . . . . . . . . . 156

Figure 4.60 Wall-normal profiles of Reynolds stresses comparing statistics of sampled data (black), POD reconstructions (orange), and LODS with periodic recalibration (blue), and the spatially averaged profile from the full DNS (black,dashed). . . . . . . . . . . 158

Figure 4.61 Frequency spectra of the fluctuating velocity field at $y^{+}=29.89$. In each subfigure are spectra of streamwise velocity $\left(E_{u u}\right.$, blue), wall-normal velocity ( $E_{v v}$, orange), and spanwise velocity $\left(E_{w w}\right.$,

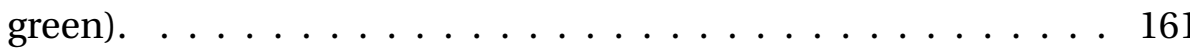

Figure 4.62 Frequency spectra of the fluctuating velocity field at $y^{+}=371.6$. In each subfigure are spectra of streamwise velocity $\left(E_{u u}\right.$, blue), wall-normal velocity ( $E_{v v}$, orange), and spanwise velocity ( $E_{w w}$,

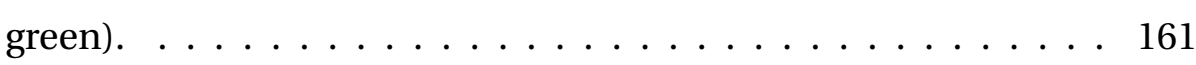

Figure 4.63 Mean velocity in the wake of an isolated wind turbine. . . . . . . 165

Figure 4.64 Reynolds normal stresses. . . . . . . . . . . . . . . . . . . . 167

Figure 4.65 Reynolds shear stresses. . . . . . . . . . . . . . . . . . 168

Figure 4.66 Distribution of energy in the POD mode basis according to eigenvalues $\lambda_{n}$. Normalized values appear in (a); the normalized cumulative summation of eigenvalues is shown in (b) with thresholds corresponding to 50, 75 and $95 \%$ of the integrated turbulence kinetic energy in the domain. . . . . . . . . . . . . . . 169 
Figure 4.67 Example POD modes from wind turbine wake used in low-order model. . . . . . . . . . . . . . . . 170

Figure 4.68 Time evolution of POD coefficients (black) and least-squares fit (blue). ..................... 171

Figure 4.69 Constant 4.69(A), linear 4.69(B), and quadratic parameters 4.69(C) and 4.69(D) coupling the mode coefficients in the LODS. 173

Figure 4.70 POD coefficients (black) and predictions from the coupled ODEs in the low-order dynamical system (blue). . . . . . . . . . . . 175

Figure 4.71 Work flow demonstrating the input/output map of the dynamical system with periodic recalibration from the effective inflow velocity. . . . . . . . . . . . . . . . . . 177

Figure 4.72 Effective incoming velocity. Time used to define transfer functions shown in blue. Validation time shown in black. . . . . . . 177

Figure 4.73 Comparison of LODS coefficient predictions with recalibration at a variety of periods. In each subfigure from top, $T_{p} \in$ $[200,100,50,25]$ seconds. . . . . . . . . . . . . . . 180

Figure 4.74 Fit between coefficients predicted by dynamical model and their respective values from POD. Coefficients for each mode $i$ are tested for a range of time periods $T_{p}$ (in seconds). . . . . . . 181

Figure 4.75 LODS coefficient predictions periodically recalibrated with initial conditions via effective inflow velocity and transfer functions. 182 
Figure 4.76 Comparison of velocity signals at locations downstream of the wind turbine. Blue lines are $\hat{u}_{\text {wake }}$ integrated over the swept area of the rotor, black lines are validation data from the LES, green lines are produced with POD . . . . . . . . . . . . 183

Figure 4.77 Wake model Reynolds normal stresses . . . . . . . . . . . . . . 184

Figure 4.78 Wake model Reynolds shear stresses . . . . . . . . . . . . . . 185

Figure 4.79 Schematic of wind turbine array LES indicating the area of focus for reduced order modeling. . . . . . . . . . . . . . 190

Figure 4.80 Detail view of a single wake block within the simulated array. Effective inflow velocity is to be calculated with the transferfunction calibrated LODS. . . . . . . . . . . . . . . . . 191

Figure 4.81 Mean velocity (A), streamwise normal stress (B), and primary shear stress $(\mathrm{C})$ in wake IV. . . . . . . . . . . . . . . . . 192

Figure 4.82 Parameters coupling dynamic modes of wake IV. . . . . . . . . 193

Figure 4.83 POD (black) and LODS (blue) coefficients corresponding for the 12-mode system in wake IV. . . . . . . . . . . . . . . . 194

Figure 4.84 Comparison of mode coefficient from POD and that produced from the open-loop transfer functions. Only coefficients corresponding to modes $1-5$ are shown. . . . . . . . . . . . 195

Figure 4.85 Time-series comparison of calculated (black) and modeled (blue) coefficients after transfer function recalibration. . . . . . 197

Figure 4.86 Effective inflow velocity signals provided to a wind turbine in the fifth row. The trend in black is calculated from the LES directly, the trend in blue results from the LODS model for wake IV. . . . 198 
Figure 4.87 Mode coefficients for wake V. Series derived from the POD are shown in black, series from the wake V LODS are shown in blue. 200

Figure 5.1 Example control scheme for a wind turbine in a large array. This scheme could be nested and account for many rows of wind turbines to provide global array control with very few inputs. . . . . 210 


\section{Chapter 1}

\section{Introduction}

Wind energy currently represents a significant portion of the global energy production and is due to increase sharply in the coming decades. A recent study by the United States Department of Energy (Vision, 2015) identifies many benefits from increased wind energy development ranging from increased national security to better and more affordable public health and water scarcity resilience. Currently, the U.S. aims to generate $20 \%$ of the nation's electricity from wind power by 2030 (Lindenberg et al., 2008), and up to $35 \%$ by 2050 ; an ambitious goal that requires acceleration of the current rate of wind project developments including both on-shore and off-shore wind parks (Van Cleve and Copping, 2010).

The US wind industry installed 8,598 MW of new capacity in the year 2015, a 77\% increase over the previous year and among the largest growth years in history. The new installations bring total wind power capacity in the US up to $74,471 \mathrm{MW}$, enough installed capacity to account for the average power consumed by 20 million American homes, according to the Global Wind Energy Council (Fried et al., 2015; Global Wind Energy Council, 2014). Wind energy currently represents the fastest growing utilityscale power investment in the US. In the last 5 years, wind energy has supplied $30 \%$ of all new power capacity additions. In 2015 wind energy alone accounted for $41 \%$ of new 
capacity additions in the US. Recent years have seen an impressive $66 \%$ decrease in the overall cost of wind energy according to the Department of Energy's 2014 Wind Technologies Market Report (Smith et al., 2015). The decrease in costs of wind energy come from significant advancements in research and technology as well as a large increase in domestic manufacturing and investment. Since 2008, over $\$ 114$ billion in private investment has flowed into the US wind industry and is one of the leading choices for new generation by utilities.

Regrettably, many wind energy systems perform far below their rated levels and suffer accelerated degradation from a combination of turbulence-related phenomena, including wind turbine wake interaction, siting, and topography effects. As an example, the Biglow Canyon Wind Farm in Oregon operates at an estimated 37\% capacity factor, the value that compares actual power generated to rated peak power generation. This estimate indicates that there is a great deal of room to improve the performance of established and new wind farms. Translating into economic terms, an increase in wind power production of $1 \%$ in Oregon alone corresponds to approximately $\$ 3.7$ million per year (Oregon average power cost is $\$ 0.079 / \mathrm{kWh}$, total 2015 production is 389 GWh).

Plans outlined by the Department of Energy to ramp up wind energy development will require research to address known and emerging difficulties with power generation, storage, and distribution. Wind turbines often perform below expected or nominal capacities due to an incomplete understanding of atmospheric forcing and the interaction of wind turbine wakes in large arrays (Wharton and Lundquist, 2012). New modeling platforms are necessary to develop the next generation of control strategies for wind farms (Brunton and Noack, 2015). A necessary step in this direction is to 
characterize wind turbine wakes in an efficient way such that computationally inexpensive and robust predictive models may be generated. The complexity of the turbulent flow in a wind turbine array canopy has proven to be out of reach with firstorder momentum-balance type models (Crespo et al., 1999). On the other end of the spectrum, schemes including large eddy simulation (LES) are capable of resolving the physics of the flow, but are computationally expensive and remain confined to academic settings taking place on supercomputers (Laan et al., 2015). Expensive models do not allow the rapid iteration necessary to account for the range of operating conditions seen in many wind farms. The current work aims to characterize a wind turbine wake in an array and to identify the dynamics necessary to formulate low-dimensional models leading to effective tools in wind turbine array control and design.

Individual wind turbine wakes have been investigated in great detail (see e.g. Ainslie (1988); Whale et al. (1996); Snel (2003); Vermeer et al. (2003)). One experimental study by Chamorro and Porté-Agel (2009) found that characteristics in the wake of an individual wind turbine depend on the surface roughness in the approach flow, underpinning the importance of atmospheric forcing and environmental conditions on the performance of wind turbines. The spatial distribution of the velocity deficit and the turbulence intensity, important factors affecting turbine power generation and fatigue loads in wind turbine rotor blades and nacelles, exhibit asymmetry in the wakes. According to that study, the distribution and intensity of turbulence in the wake is stronger over rough surfaces, affecting the atmospheric flow at turbine height. Studies of individual wind turbines form a foundation from which the aerodynamics of large wind turbine arrays may be investigated and understood.

The turbulent flow in a wind turbine array (WTA) canopy represents a real chal- 
lenge to characterize and has received a great deal of attention in recent years. WTA flows combine asymmetrical wind turbine wakes with environmental conditions that change drastically over a wide range of scales, representing times up to diurnal and annual scales, and length scales from the chord length to the size of wind farms. In wind farms, individual wakes interact with one another and further change the behavior of the wind turbines. In very large arrays, wind turbines are necessarily placed in the wakes of upstream devices. Limitations on turbine spacing are imposed by economic constraints in an effort to maximum the financial yield of each acre of land dedicated to wind power production. The energy balance for an isolated wind turbine differs from that of a turbine within a large array; most notably, for wakes in large arrays, turbulent fluxes of mean flow kinetic energy are expected to be order one quantities, which is not the case for an isolated turbine. The specific nature of wake evolution and interaction in WTAs remains a complex flow to quantify and predict (Frandsen et al., 2006; Global Wind Energy Council, 2014).

WTAs are considered 'fully developed' when flow statistics in wakes exhibit periodicity from row to row (Chamorro and Porté-Agel, 2011b), generally considered a good approximation beyond the fourth row of turbines in a regular Cartesian WTA arrangement. Wake flow statistics beyond the fourth row of turbines are assumed to approximate successive rows in the array (Calaf et al., 2010; Chamorro et al., 2012; Meyers and Meneveau, 2012). For large WTAs, the kinetic energy ultimately extracted by turbines must be transported from above the array to the canopy layer (Cal et al., 2010; Calaf et al., 2010). The resupply of mean flow kinetic energy to the momentum-deficit central to wind turbine wakes is largely accomplished by turbulence. The shear layer at the border of each wake exhibits strong gradients in mean velocities and significant 
turbulence shear stresses. Also important to the energy balance in the fully developed turbine array is the production of turbulence kinetic energy, especially near to the wind turbine rotor. Although short-lived in the wake, this term is often related to the amount of mean flow kinetic energy converted into turbulence and made unavailable to successive turbines in the array.

Energy produced by a wind turbine was correlated to the difference in the flux of turbulence kinetic energy at top and bottom tips of the rotor blades by Cal et al. (2010), providing a means of estimating energy production from wake turbulence and further emphasizing the importance of energy fluxes in turbine wakes. In large arrays especially, the global kinetic energy entrainment is downward from above the wind turbine canopy into the wakes. The formulation of the flux of kinetic energy suggests that energy containing structures, especially those associated with the top tip of the rotor area, are responsible for the entrainment of energy downward into the main turbine canopy area (Hamilton et al., 2012). Investigation of the component quantities of the flux of kinetic energy is of continued interest in studies pertaining to wind turbine arrays. Previous experimentation in wind farms has shown that the flux of kinetic energy is mainly expressed by the energetic turbulent structures. Large-scale dominance of energy fluxes was shown by way of the premultiplied spectral density (Hamilton et al., 2012) and the proper orthogonal decomposition (Hamilton et al., 2015b).

Coherent turbulence structures contributing to the downward flux of kinetic energy are often attributed to tip-vortices issuing from the rotor blades of a turbine. The rotational nature of the blades implies that the helical tip-vortices transport mean flow energy with a periodicity fixed by the tip-speed ratio of the rotor. Structures related to the phase orientation of the rotors have been investigated for wind turbines by $\mathrm{Hu}$ 
et al. (2012) and Lignarolo et al. (2014) illuminating the vortex development in wakes from isolated horizontal axis wind turbines. Tip vortices produced by a two-bladed rotor were shown to persist up to 2.5 rotor diameters into the wake for an idealized inflow, where the turbulence intensity was constrained to approximately $2 \%$ (Lignarolo et al., 2014). Placing a wind turbine in the simulated atmospheric boundary layer as in Hu et al. (2012) more accurately reproduced the aerodynamics of wind turbines in the field, and in doing so demonstrated phase-dependent structures only near the model wind turbine. When the inflow to the turbine deviates from idealized conditions, vortical structures become less coherent. Wakes in WTAs evolve under greatly enhanced turbulence, further obscuring these structures. Ensemble averaging statistics with respect to phase orientation of the rotor served only to obscure vortical structures visible in instantaneous velocity snapshots. Although phase dependence may not be critical for wake dynamics beyond a single rotor diameter, the rotation of the blades is correlated with wake and flux asymmetry as noted by Meyers and Meneveau (2013).

Conditional statistics has a long history in turbulence research, especially with respect to periodic forcing, since the original formulation of the triple decomposition of the velocity field (Reynolds and Hussain, 1972). Deviations of phase-averaged flows from the ensemble averaged flow are termed deterministic stresses to distinguish them from the stochastic turbulence field. Regarding turbomachinery, deterministic stresses, which are themselves phase-independent features, have been shown to be significant to the overall energy balance, especially with respect to gas turbines, shown by numerical simulation in Adamczyk (2000). Further work investigating phase-related flow effects in gas turbines by Uzol et al. (2003), revealed that the deterministic contribution to the stress field is of the same order of magnitude as the turbulence. It 
was shown that the production of turbulence kinetic energy by deterministic stresses was the dominant source of energy relevant to the flow (Uzol et al., 2003). While the flow in a gas turbine is significantly different than that of a wind turbine wake, the triple decomposition and deterministic stresses may still reveal insights into the phase-dependent and average-passage dynamics following the rotor blades. The significance of deterministic stresses in turbomachines that add energy to the flow is expect to differ from wind turbine wakes, which actively remove energy from atmospheric flows.

With the increase of readily available computational power, numerical simulations of wind arrays have become more complex, as in Barthelmie et al. (2007); Frandsen et al. (2006); Rathmann et al. (2007). Many models rely on self-similar velocity deficit profiles obtained from experimental and theoretical work (Emeis and Frandsen, 1993; Milborrow and Ainslie, 1992). The growth rate of wakes in turbine arrays is generally attributed to the level of turbulence encountered, which is amplified by the mean shear in the wind turbine canopy layer. Typically, the momentum deficit predicted in wind turbine wakes is obtained from global conservation principles. One of the main advantages of studying the WTA canopy through numerical simulations is that physical phenomena that are out of reach in many experiments, such as Coriolis forcing and thermal stratification, may be included.

Most simulations of large wind farms consider turbines as a series of roughness elements, solid bodies or disks that increase the effective surface roughness for the atmospheric boundary layer (ABL) and decrease mean wind speeds near the ground. The actuator disc model was introduced to large eddy simulations of wind turbine arrays by Sørensen et al. (1998), and is still in wide use within the academic setting. Spacing opti- 
mization undertaken by Meyers and Meneveau (2012) addresses the problem of windturbine spacing in wind farms, where the optimal spacing is a product of economical constraints and the performance of turbines within the array. The study showed that the optimal spacing for turbines based on performance may be as large as 15 rotor diameters in the streamwise direction rather than the 6 or 7 diameters typically found in real installations. Rotational effects are introduced to the actuator disc model by application of a tangential force in the disc, with significant effects on the kinetic energy ultimately extracted by the rotors (Meyers and Meneveau, 2013).

An important element in the dynamics of the wind turbine canopy layer introduced by swirl imparted to the flow from the passage of the rotor blades is anisotropy in the Reynolds stress tensor (Snel, 2003). To account for turbulence anisotropy, more sophisticated wind farm simulations opt for computationally expensive models. Schemes balancing the transport equations of the Reynolds stresses are far more effective at representing the anisotropy of turbulence than the more computationally efficient RANS codes that balance the transport of turbulence kinetic energy and either dissipation ( $k \epsilon$ models), or vorticity ( $k \omega$ models) (Rogallo and Moin, 1984).

Turbulence anisotropy is frequently characterized through anisotropy tensor invariant analysis and is seen in simplifying assumptions, boundary conditions, and theoretical development (Biferale and Procaccia, 2005; Kim et al., 1987). The second and third mathematical invariants of the normalized Reynolds stress anisotropy tensor together describe the possible states of anisotropy seen in realizable turbulence, represented with the anisotropy invariant map (AIM, or Lumley triangle) introduced by Choi and Lumley (2001). Theoretical development of the anisotropic state of turbulence has been further employed in predictive models of turbulence often seen in the 
form of boundary conditions, as for wall-bounded turbulence, seen in direct numerical simulations such as Graham et al. (2013). Anisotropy tensor invariants are crucial to the model of Rotta (1951), wherein the rate of return to an isotropic state is linearly proportional to the degree of anisotropy in a turbulence field. The Rotta model forms the basis of many second-order closure schemes such as the explicit algebraic models of turbulence as presented in Menter et al. (2012) and Rodi and Bergeles (2012).

Anisotropic turbulence evolving in a flat-plate boundary layer was detailed by Mestayer (1982), confirming that local isotropy exists in the dissipative range of scales, typically at scales smaller than twenty times the Kolmogorov microscale. Local isotropy at small scales is generally accepted at sufficiently high Reynolds number, provided that an inertial subrange separates the energetic scales from the dissipative ones. It was further shown by Smalley et al. (2002) and Leonardi et al. (2004) that surface characteristics of the wall influence the balance of turbulent stresses, and subsequently the invariants of the anisotropy tensor. Turbulent stresses tend toward isotropy in boundary layers evolving over rough surfaces more so than over smooth walls.

Smyth and Moum (2000) found that anisotropy in large-scale turbulence generates Reynolds stresses that contribute to the extraction of energy from the atmospheric boundary layer. Computational work detailing the anisotropy of turbulence in the wakes of wind turbines has been undertaken by Gómez-Elvira et al. (2005) and Jimenez et al. (2007). Both studies employ second-order closure schemes with explicit algebraic models for the components of the turbulent stress tensor. Recent experimental work (Hamilton and Cal, 2015) explored the anisotropy in wind turbine arrays wherein the rotational sense of the turbine rotors varied. The importance of anisotropy in the wind turbine wake was shown through correlations of the flux and production to the 
anisotropy tensor invariants. Flux of mean flow kinetic energy downward from above the canopy was correlated with turbulence characterized by a single dominant component. In contrast, flux upward from below the turbine rotor was correlated with turbulence showing two co-dominant components.

Alternate means of characterizing turbulence evolving in wind turbine wakes include numerical or modal decomposition methods. Of these, the proper orthogonal decomposition (POD) is frequently employed due to its optimality in describing energetic structures in a turbulent flow. Since its introduction to the field of fluid mechanics by Lumley (1967), the POD has evolved considerably, most notably by Sirovich (1987), who, along with advancements in particle image velocimetry (PIV) technology, pioneered the method of snapshots. This widely used variant of the POD capitalizes on spatial organization of data resulting from experimental techniques such as PIV and numerical simulations.

The snapshot POD has been applied to wind turbine wakes by Hamilton et al. (2015b), wherein large structures were identified as being responsible for most of the flux of kinetic energy. It was confirmed through the POD and LES that an infinite array of wind turbines is subject to very large structures, on the order of spacing of devices in the array, that have an influence on global performance (VerHulst and Meneveau, 2014). Such structures in a wind farm were similarly detailed by Sørensen et al. (2015), where it was shown that select POD modes relate spatial development of the wake. Lignarolo et al. (2015) used snapshot POD to filter data and distinguish tip vortices shed by wind turbine rotors from the background turbulence. Results there are used to argue that that the presence of strong coherent fluctuations and structures in the near wake does inhibits the wake from mixing with the outer flow. Another recent study 
(Bastine et al., 2015) combined the snapshot POD with preprocessing of PIV images to distinguish turbulent features in the wake from those arising from the atmospheric boundary layer.

The optimality of the POD in describing the energy of a flow provides a direct means of filtering velocity measurements (Hasselmann, 1988). The reconstruction of a velocity signal or turbulent stress tensor may be accomplished using a truncated set of POD modes in linear combination. Filtering in this manner requires establishing a threshold of turbulence kinetic energy according to the eigenvalues associated with each POD mode. Because the POD organizes modes according to the energy they express in the flow, structures contributing energy outside the established threshold are excluded. Additionally, the eigenvalue decomposition sorts random and incoherent noise to the higher mode numbers, thus filtering resultant reduced order representations of the flow (Graham et al., 1999). The kernel of the POD is most commonly the two-point spatial correlation tensor and may be reconstructed directly from the POD modes and associated eigenvalues.

A relatively recent modification of the POD models was proposed by Noack et al. (2003) allowing for variation in the mean flow. Transient behavior is usually missed by the POD, as the kernel is constructed with correlations of the fluctuating velocity only. Relatively recent methodological development with the proper orthogonal decomposition includes iterative application of POD to similar sets of data. Siegel et al. (2007, 2008) showed that POD bases of identical size for multiple datasets in a cylinder wake exhibit variation at fixed mode number following the emerging from the natural variation in vortex shedding; modes of like rank exhibit subtle differences between shedding periods that can be characterized by secondary application of the POD. This nested ap- 
proach is referred to simply as the double POD (DPOD). The span of like modes were characterized by a large common projection and a set of corrections, termed 'shift' modes, whose significance is described by their respective amplitudes.

Large scales, such as those favored by low-rank POD modes, express the most energetic and the least isotropic, turbulence structures. Reconstruction of velocity fields or the Reynolds stress tensor using a reduced mode basis necessarily excludes energy from the flow, typically associated with smaller-scale turbulent scales or those not coherent in time, regardless of their potential dynamical importance (Kostas et al., 2005; Tutkun et al., 2008). Local and small-scale isotropy is expected in the dissipative range of turbulent scales or far from any bounding geometry of the flow, as in the outer boundary layer (Rotta, 1951) or far into a wake (Gómez-Elvira et al., 2005; Saddoughi and Veeravalli, 1994; Townnsend, 1948). Statistics produced with reduced order models are structurally representative of the full flow but exhibit a decreased range of magnitudes due to the exclusion of turbulence kinetic energy in intermediate and high mode numbers shown for wind turbine wakes in Hamilton et al. (2015b).

Reduced-order flow descriptions of this type aim to capture important turbulent flow features while limiting the number of degrees of freedom and complexity of the flow. Error propagation through POD basis truncation has been explored to a limited degree (Białecki et al., 2005; Noack et al., 2003; Rempfer, 2000; Tröltzsch and Volkwein, 2009) for the purposes of low-dimensional modeling through Galerkin projection. Absent from the literature, however, is any discussion relating the low-order description of the flow and the associated anisotropy tensor invariants. As the anisotropy of the turbulence field can play a large role in turbulent fluxes (Pope, 2000; Hamilton and Cal, 2015), a detailed discussion of the anisotropic state of the resulting turbulence field is 
important to the development of future POD low-dimensional models.

Low-order modeling describes a wide range of approaches used to simplify the Navier-Stokes (NS) equations in order to capture or simulate the important dynamics for a given flow with an effective use of computational resources. An attractive extension of the POD, the Galerkin projection, is widely used to perform this model reduction, resulting a minimal set of ordinary differential equations (Noack et al., 2003). The POD-Galerkin procedure was first used in turbulent shear flow modeling by Aubry et al. (1988), and has since been extended to many flow scenarios. Regrettably, the POD's ability to represent the dynamics of a system is quite sensitive to transient behavior; deviations of the system from the reference frame of the POD quickly result in divergence of the low-order dynamical system (LODS) (IEE, 2005; Noack et al., 2005; Rapún and Vega, 2010). Previous work with correction factors to the Galerkin systems (GS) indicates that correction factors that keep the dynamics within the bounds of the original system are quite effective at introducing stability (Tadmor et al., 2011). Stability may also be introduced by treatment of the pressure term as pointed out by Rempfer (2000); Noack et al. (2005). Pressure correction is especially important in compressible flow scenarios such as acoustic fields and transsonic flows (Fike, 2013).

To perform a Galerkin projection, a large database is necessary in order to compute the inner products and spatial gradients of constituent modes. Consequently, LODS derivation are most effectively performed from numerical simulation data or need some strong closure assumptions, particularly in an experimental framework (Braud et al., 2004). The amount of data required is often a difficult constraint to overcome, and may defeat the purpose of reduced-order modeling. Another approach to the LODS was presented in Perret et al. (2006b) wherein the coefficients from the POD 
are used directly in the formation of the dynamical system. In this approach, simultaneous realizations of the coefficients and their respective time derivatives are required to resolve the evolution of the system. So-called gray-box control systems of this nature are pointed to as a robust choice moving forward in closed loop control development, especially for power systems such as WTAs (Brunton and Noack, 2015).

The rapid development of wind energy points to the need for new tools in order to optimize the control and optimization of large WTAs. Turbulent energy fluxes in the wakes are difficult to accurately resolve with computationally inexpensive models due to incomplete characterization of the critical wake dynamics. The POD is a well-tested tool used to identify energetic turbulence structures that can be used to seed dynamical models capable of predicting wind turbine wakes. To date, these sorts of models rely mainly on the Galerkin projection, not extensively used in wind energy as the complexity of the flow typically leads to instabilities in resulting dynamical system models. Low-order dynamical systems evolving from the dynamic elements of the POD present a promising path forward. Such models combine with correction factors that significantly increase their accuracy, reducing the flattening of turbulence, and account for energy excluded in the reduction of degrees of freedom.

Research presented below expands on the triple decomposition of velocity measurements in planes spanning a wind turbine wake in order to assess the phaseaveraged and deterministic contributions to the energy balance. The flux of kinetic energy is formulated with the phase-averaged and deterministic stresses. The rotors of a wind turbine do not impart phase-dependent variations to the filed that persist in the mean flow, in contrast to other forms of turbomachinery. An extension of the triple decomposition is applied to the turbulence stress tensor showing the contribution of 
third order statistics to be significant in the flow field.

Turbulence structures in wind turbines wakes are isolated with POD and shown to evolve spatially. The DPOD is reformulated such that it may apply along a spatial coordinate allowing for an additional layer of basis reduction. Estimates of flow statistics are then augmented by way of a correction factor, introduced as a tensor of coefficients effectively compensating for energy truncated from the modal bases. Error propagation through the POD basis is explored both in terms of the turbulence field and in terms of the anisotropy tensor invariants. Low-order descriptions are found to exaggerate the anisotropy of a given flow; modes excluded from the truncated POD basis supply highly isotropic turbulence. Severe basis truncations are unable to reproduce three dimensional turbulence on their own. With the aid of correction terms, more accurate and realistic turbulence is produced including three dimensionality, and flow description errors are significantly reduced.

The present dissertation is composed of work reproduced from several published or forthcoming articles. These articles have been merged into a single manuscript, removing or combining repeated content, including the literature review, theory, and experimental design. Concluding remarks serve as the discussion following each set of results section. The final chapter of the dissertation includes overarching conclusions drawn from the presented work as a whole. An outlook provides perspective on points of the presented work deserving further investigation as well as future research thrusts and opportunities. 


\section{Chapter 2}

\section{Theory}

\subsection{Equations of motion}

The momentum balance for a wind turbine canopy or wake is described by the Reynolds averaged Navier-Stokes equations as,

$$
U_{j} \frac{\partial U_{i}}{\partial x_{j}}=-\frac{1}{\rho} \frac{\partial P}{\partial x_{i}}-\frac{\partial \overline{u_{i} u_{j}}}{\partial x_{j}}-\bar{f}_{x}
$$

In Equation (2.1) the time derivative term has been omitted through ensemble averaging of the equations. The time dependence of the fluid motion and momentum are assumed to be null when averaged over time. It is further assumed that all measurement locations described in the system are sufficiently far from solid bodies to neglect viscous terms in the local momentum balance. The term $\bar{f}_{x}$ represents the thrust force of the turbines in the flow, acting primarily in the streamwise direction, leading to the subscript $x$ rather than the standard index; $\rho$ is the density of the fluid; $P$ is taken as the mean pressure field. In all following equations, the coordinates are designated as $x$ (streamwise), $y$ (wall-normal), and $z$ (spanwise) directions, respectively. Capital letters indicate mean quantities and lower case letters refer to zero-centered fluctuations about the mean. An overline is employed to indicate that an ensemble average of the 
product of two quantities has been taken.

Multiplying by the mean velocity yields the mean mechanical energy equation

$$
U_{j} \frac{\partial \frac{1}{2} U_{i}^{2}}{\partial x_{j}}=-\frac{1}{\rho} U_{i} \frac{\partial P}{\partial x_{i}}+\overline{u_{i} u_{j}} \frac{\partial U_{i}}{\partial x_{j}}-\frac{\partial \overline{u_{i} u_{j}} U_{i}}{\partial x_{j}}-U_{i} \bar{f}_{x}
$$

The left hand side of the Equation (2.2) is comprised of the convective terms and is balanced on the right by the power added to the flow through pressure gradients, the production of turbulence kinetic energy, and the flux of turbulence kinetic energy, respectively. The last term of Equation (2.2) is a sink representative of the power removed from the flow by the action of a wind turbine rotor, $U_{i} \bar{f}_{x}$.

Of critical consideration in the global energy balance is the Reynolds stress tensor, $\overline{u_{i} u_{j}}$. This tensor expresses the balance of fluid stresses at any given measurement location and in Equation (2.2) is a component of both the kinetic energy flux and the turbulence production. The symmetric stress tensor, $\overline{u_{i} u_{j}}$, is given by

$$
\overline{u_{i} u_{j}}=\left[\begin{array}{lll}
\overline{u^{2}} & \overline{u v} & \overline{u w} \\
\overline{v u} & \overline{v^{2}} & \overline{v w} \\
\overline{w u} & \overline{w v} & \overline{w^{2}}
\end{array}\right] .
$$

The Reynolds stress tensor is symmetric, arising from the Reynolds averaging process. Terms on the diagonal are normal stresses and off-diagonal terms are shear stresses in the turbulent stress field. The turbulent kinetic energy $k$ is defined as half of the trace of $\overline{u_{i} u_{j}}$,

$$
k=\frac{1}{2}\left(\overline{u^{2}}+\overline{v^{2}}+\overline{w^{2}}\right) .
$$




\subsection{Phase dependent statistics}

The total Reynolds stress shown in equation (2.3) is ensemble averaged over all measurements, regardless of phase angle $\alpha$ of the turbine rotor. Any quantity conditionally averaged over time is considered independent of phase. In the following development, angle brackets indicate that a quantity has been averaged with respect to the coordinate or parameter following as a subscript. |Phase-dependent quantities are conditionally averaged as $\left\langle\tilde{o}_{i}\right\rangle_{\alpha}=\left\langle\tilde{o}_{i}\right\rangle\left(x_{i}, \alpha\right)$. Averaging phase-dependent values over the ensemble of all phase angles yields an approximation of the time-averaged quantity, $\overline{\tilde{o}_{i}}=\overline{\left\langle o_{i}\right\rangle_{\alpha}}=O_{i}$. The deviation of phase-averaged quantities from their respective time-averaged values is expressed as the difference between the two quantities and is denoted with a double-prime,

$$
u_{i}^{\prime \prime}=U_{i}-\left\langle\tilde{u}_{i}\right\rangle_{\alpha}
$$

These deviations may be multiplied and ensemble averaged over the period of the wind turbine rotor motion to acquire deterministic stresses (Adamczyk, 2000; Reynolds and Hussain, 1972).

$$
\overline{u_{i}^{\prime \prime} u_{j}^{\prime \prime}}=\overline{\left(U_{i}-\left\langle u_{i}\right\rangle_{\alpha}\right)\left(U_{j}-\left\langle u_{j}\right\rangle_{\alpha}\right)}
$$

The total stress field is described by the sum of the ensemble of phase-averaged Reynolds stresses and a deterministic component that arises from deviations between phase-averaged mean velocities and the average-passage value. Similarly, the total flux of kinetic energy may be decomposed into turbulent (phase-dependent) and deterministic (phase-independent) contributions. Current literature suggests that the com- 
ponent of the flux tensor responsible for global kinetic energy entrainment into the array involves the streamwise-wall-normal fluctuating velocities, combined with the streamwise mean flow,

$$
F_{12}=-\overline{u v} U=-\left({\overline{\langle u v\rangle_{\alpha}}}+\overline{u^{\prime \prime} v^{\prime \prime}}\right){\overline{\langle U\rangle_{\alpha}}}_{.}
$$

Locally, high-momentum flow is introduced to the wake of an individual wind turbine through streamwise-spanwise turbulence at a similar order of magnitude as $F_{12}$,

$$
F_{13}=-\overline{u w} U=-\left({\overline{\langle u w\rangle_{\alpha}}}+{\overline{u^{\prime \prime} w^{\prime \prime}}}_{\overline{\langle U\rangle}}\right.
$$

Highly variable flow in the turbine canopy is not necessarily expected to demonstrate significant deterministic contributions to the stress field due to mixing effects independent of the phase orientation of the rotor. Here, the root-mean-square of the deviation of the phase-averaged stress field from the time-averaged Reynolds stresses are termed tertiary stresses and defined as,

$$
\overline{u_{i}^{\prime \prime \prime} u_{j}^{\prime \prime \prime}}=\sqrt{\overline{\left(\left\langle u_{i} u_{j}\right\rangle_{\alpha}-\overline{u_{i} u_{j}}\right)^{2}}}
$$

Normal components of the tertiary stress tensor are the deterministic analog to the turbulence intensities along the coordinate directions. The physical significance of the off-diagonal terms is less intuitively obvious, but are taken here to be of equal importance to the flow as the deterministic stresses from equation (2.6). 


\subsection{Anisotropy of the turbulent stress tensor}

The particular balance of terms in the Reynolds stress tensor is important when considering turbulent transport phenomena. In an ensemble sense, isotropic turbulence does not contribute to a net flux in any particular direction, as what is instantaneously transported in one direction would be balanced by an equal and opposite transport at a later time (Pope, 2000). To quantify deviation from an isotropic stress field, it is useful to define the Reynolds stress anisotropy tensor $\breve{a}_{i j}$, here following the development by Rotta (1951),

$$
\breve{a}_{i j} \equiv \overline{u_{i} u_{j}}-\frac{2}{3} k \delta_{i j}
$$

where $\delta_{i j}$ is the Kronecker delta. Typically, the anisotropy tensor is normalized by the turbulence kinetic energy,

$$
\begin{aligned}
& \breve{b}_{i j}=\frac{\breve{a}_{i j}}{2 k}=\frac{\overline{u_{i} u_{j}}}{\overline{u_{k} u_{k}}}-\frac{1}{3} \delta_{i j}
\end{aligned}
$$

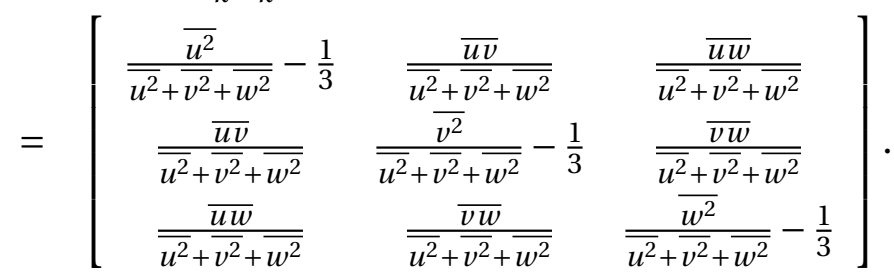

The first invariant of the normalized anisotropy tensor, the trace of $\breve{b}_{i j}$, is identically zero as a consequence of its normalization. The traces of $\breve{b}_{i j}^{2}$ and $\breve{b}_{i j}^{3}$ are related to the second and third invariants ( $\eta$ and $\xi$ ) of the anisotropy tensor as, 


$$
\begin{aligned}
& 6 \eta^{2}=\breve{b}_{i i}^{2}=\breve{b}_{i j} \breve{b}_{j i}, \\
& 6 \xi^{3}=\breve{b}_{i i}^{3}=\breve{b}_{i j} \breve{b}_{j k} \breve{b}_{k i} .
\end{aligned}
$$

Invariants of the normalized Reynolds stress anisotropy tensor are related to the degree of anisotropy $(\eta)$ and the characteristic shape associated with the particular balance of stresses $(\xi)$.

Invariants of $\breve{b}_{i j}$ are frequently plotted against one another in the anisotropy invariant map (AIM), also known as the Lumley triangle (Choi and Lumley, 2001). Theoretical limits and special forms of turbulence are shown as vertices or edges of the triangle in figure 2.1. These cases are often used in scale analysis of flows and represent theoretical limits of 'realizable' turbulence. See table 2.1 for descriptions of each state of turbulence in terms of their respective invariants. The invariants $\eta$ and $\xi$ may also be defined with the eigenvalues of the normalized Reynolds stress anisotropy tensor. Such eigenvalues are interpreted as the spheroidal radii of shapes that characterize the turbulence anisotropy and correspond to the limits shown in the Lumley triangle (see e.g. Hamilton and Cal (2015)). Characteristic shapes for special cases of turbulence are noted in table 2.1.

Special cases of turbulence outlined in Table 2.1 are often used in scaling and theoretical development but are not typically observed in real turbulence. Perfectly isotropic turbulence occurs when the deviatoric of the Reynolds stress tensor (the anisotropy tensor) is null and $\xi=\eta=0$. Due to the mathematical relationship between the invariants given by equations (2.12) and (2.13), $\xi=0$ occurs only when $\eta=0$, 
Table 2.1: Limiting cases of turbulence given on the Lumley triangle in terms of anisotropy tensor invariants.

\begin{tabular}{ccc}
$\begin{array}{c}\text { State of } \\
\text { turbulence }\end{array}$ & Invariants & $\begin{array}{c}\text { Shape of } \\
\text { spheroid }\end{array}$ \\
\hline $\begin{array}{c}\text { Isotropic } \\
\text { Two-component } \\
\text { axisymmetric } \\
\text { One-component } \\
\text { Axisymmetric }\end{array}$ & $\xi=\eta=0$ & Sphere \\
(one large eigenvalue) & $\xi=\eta=\frac{1}{6}, \eta=\frac{1}{6}$ & Disk \\
Axisymmetric & $\xi=\eta$ & $\begin{array}{c}\text { Line } \\
\text { Prolate } \\
\text { spheroid } \\
\text { One small eigenvalue) } \\
\text { Two-component }\end{array}$ \\
\hline
\end{tabular}

at the perfectly isotropic condition. The upper limit in the Lumley triangle describes two-component turbulence, where $\eta=\left(1 / 27+2 \xi^{3}\right)^{1 / 2}$.

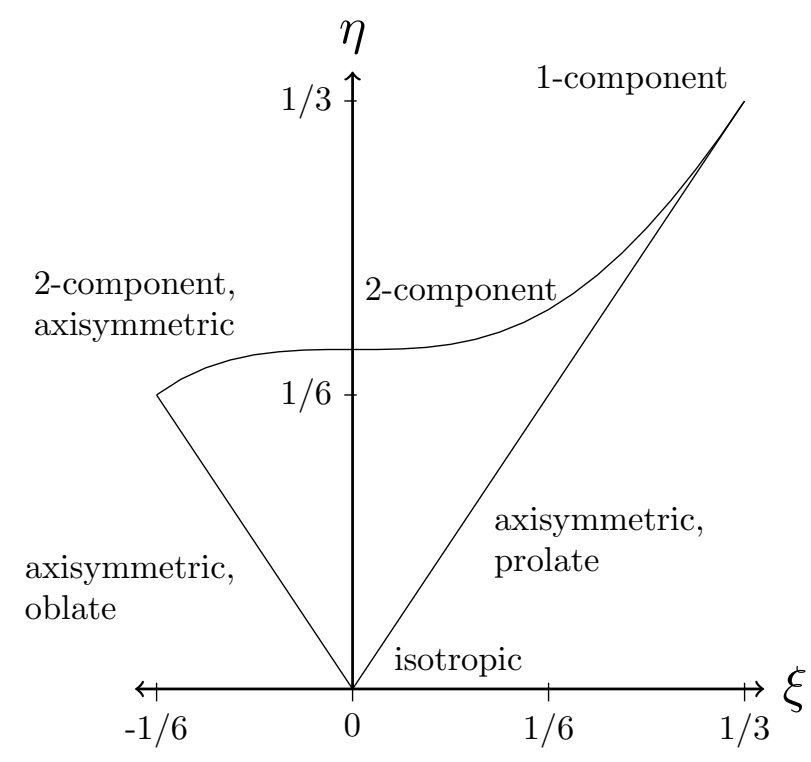

Figure 2.1: Lumley triangle showing limits of realizable turbulence according to the anisotropy tensor invariants $\eta$ and $\xi$. 
Axisymmetric turbulence is commonly observed in round jets, circular disk wakes, swirling jet, etc. The characteristic shapes associated with axisymmetric turbulence are either oblate or prolate spheroids. Oblate spheroids exhibit two eigenvalues that are of equal magnitude and one eigenvalue that is much smaller. This results in a spheroid squeezed in one direction. Prolate spheroids show the opposite effect with one eigenvalue that is of a larger magnitude compared to the other (equal or very similar) eigenvalues, resulting in a spheroid that is stretched in one direction.

One-component turbulence shows the least uniformity between components and the greatest sensitivity to rotation. Two-component turbulence, occurs as the small eigenvalue is reduced to zero, and the characteristic shape becomes an ellipse. In twodimensional axisymmetric turbulence, the characteristic shape is a circle and is invariant to rotation only along the axis defined by its null eigenvalue.

\subsection{Snapshot proper orthogonal decomposition}

Snapshot POD as follows is in the vein of the original development by Sirovich (1987) and is applied to flow data with high spatial resolution relative to temporal resolution. The outputs of the decomposition are an ordered set of modes and eigenvalues denoting the energy associated with each mode. The organized basis of modes from the POD has been described as projections common to the span of snapshots in a dataset Sirovich (1987); Hasselmann (1988); Aubry et al. (1991).

In the following development, bold math symbols represent vectorial quantities and symbols in plain text are scalar quantities. The flow field is assumed to be a stochastic function of space and time. Velocity snapshots are then denoted as $\boldsymbol{u}\left(\boldsymbol{x}, t^{n}\right)$, where $\boldsymbol{x}$ and $t^{n}$ refer to the spatial coordinates and $n^{\text {th }}$ time sample, respectively. The 
spatial correlation tensor is approximated as,

$$
\boldsymbol{R}\left(\boldsymbol{x}, \boldsymbol{x}^{\prime}\right)=\frac{1}{N} \sum_{n=1}^{N} \boldsymbol{u}\left(\boldsymbol{x}, t^{n}\right) \boldsymbol{u}^{T}\left(\boldsymbol{x}^{\prime}, t^{n}\right)
$$

where $N$ signifies the number of snapshots and the prime represents the spatial coordinate of another point in the domain.

The two-point spatial correlation tensor forms the kernel of the POD integral equation. It is assumed that a basis of $N$ modes can be written in terms of the original data as,

$$
\boldsymbol{\Phi}(\boldsymbol{x})=\sum_{n=1}^{N} A\left(t^{n}\right) \boldsymbol{u}\left(\boldsymbol{x}, t^{n}\right),
$$

where $\boldsymbol{\Phi}(\boldsymbol{x})$ is a deterministic field that has the largest projection on the stochastic velocity field in a mean square sense. The velocity snapshots combine linearly with the time-dependent coefficients $A\left(t^{n}\right)$ to form the POD modes in equation (2.15).

The POD integral equation is written as,

$$
\int_{\Omega} \boldsymbol{R}\left(\boldsymbol{x}, \boldsymbol{x}^{\prime}\right) \boldsymbol{\Phi}\left(\boldsymbol{x}^{\prime}\right) d \boldsymbol{x}^{\prime}=\lambda \boldsymbol{\Phi}(x),
$$

where $\lambda$ is the set of eigenvalues relating $\Phi$ to $\boldsymbol{R}$. Equations (2.14) and (2.15) are substituted into equation (2.16) and discretized such that the integral equation, which takes the form of an eigenvalue problem, may be solved numerically in the following form,

$$
\boldsymbol{C A}=\lambda \boldsymbol{A}
$$

In equation (2.17), $\boldsymbol{A}$ is a vector of coefficients corresponding to each snapshot in time 
and $C$ is an approximation of the correlation tensor from equation 2.14.

Solving equation (2.17) yields the set of coefficients from which the POD modes are computed according to equation (2.15). These modes are typically normalized with their own $L^{2}$-norm to form an orthonormal basis,

$$
\boldsymbol{\Phi}^{(i)}(\boldsymbol{x})=\frac{\sum_{n=1}^{N} A^{i}\left(t^{n}\right) \boldsymbol{u}\left(\boldsymbol{x}, t^{n}\right)}{\left\|\sum_{n=1}^{N} A^{i}\left(t^{n}\right) \boldsymbol{u}\left(\boldsymbol{x}, t^{n}\right)\right\|}, \quad i=1, \ldots, N
$$

Stochastic velocity fields may then be reconstructed using the eigenfunctions of the POD,

$$
\boldsymbol{u}\left(\boldsymbol{x}, t^{n}\right)=\sum_{i=1}^{N} a_{i} \boldsymbol{\Phi}^{(i)}(\boldsymbol{x}),
$$

where $a_{i}$ is a set of coefficients obtained by back-projecting the set of stochastic velocity fields onto the deterministic POD modes,

$$
a_{i}=\int_{\Omega} \boldsymbol{u}\left(\boldsymbol{x}, t^{n}\right) \boldsymbol{\Phi}^{(i)}(\boldsymbol{x}) d \boldsymbol{x} .
$$

Using a truncated set of POD modes results in a filtered turbulent flow field according to the turbulence kinetic energy associated with each mode.

The POD provides the optimal set of eigenfunctions that decompose the turbulence in a mean-squared sense into functions and eigenvalues representative of the energy. Fluctuating velocity measurements are reconstructed as in equation (2.19) and, by extension, the Reynolds stress tensor may be reconstructed directly from the POD modes according to,

$$
\overline{u_{i}(\boldsymbol{x}) u_{j}(\boldsymbol{x})}=\sum_{n=1}^{N} \lambda^{(n)} \phi_{i}^{n}(\boldsymbol{x}) \phi_{j}^{n}(\boldsymbol{x})
$$


Reconstructed Reynolds stresses in equation (4.19) are used to study quantities in the transport equation for the mean kinetic energy. The POD may be used as a means of order reduction through either equation (2.19) to reconstruct velocity snapshots filtered by truncated POD bases or by equation (4.19) in which the kernel is reconstructed directly. Statistics based on the reconstructed data using a truncated set of POD modes will be denoted in the following text with an over-ring and a subscript indicating the number of POD modes $\left(N_{r}\right)$ or percent of the total energy used in reconstruction as $\left.\stackrel{\circ}{u \nu}\right|_{N_{r}}=\sum_{n=1}^{N_{r}} \lambda^{(n)} \phi_{u}^{(n)} \phi_{v}^{(n)}$ or, following equation (2.19), $\left.\stackrel{\circ}{\boldsymbol{u}}\right|_{N_{r}}=\sum_{n=1}^{N_{r}} a_{n} \boldsymbol{\Phi}^{(n)}$.

\subsection{Double POD}

The snapshot POD outlined above is applied to dataset from each measurement location individually. The spatial development of turbulence structures in the wake is shown along the streamwise coordinate $x$. As each measurement set contains the same number of snapshots and therefore the same number of POD modes, it is safe to assume that the ordering of structures in the POD basis is similarly organized Siegel et al. $(2005,2007,2008)$. The development of $\boldsymbol{\Phi}^{(n)}(\boldsymbol{x})$ in the wake is characterized by the linear combination of sub-modal structures and corresponding amplitudes. With increasing mode number $n$, structural organization is lost in each set and fails to communicate development downstream. Energetic features as indicated by low mode numbers show correspondence between the measurement sets throughout the wake and are seen to grow, diffuse, or otherwise evolve spatially with $x$.

Applying the POD to sets of modes of common rank, that is modes at a fixed mode number $n$ from each separate POD basis, then shows the sub-modal structures, previously termed 'shift modes.' With such an application of DPOD the internal basis of 
POD modes is already time independent (dependence on time is now accounted for by the series of mode amplitudes $a_{n}$ ) and the DPOD accounts for cycle-to-cycle differences in bases. In the current formulation of the DPOD, decomposition occurs over the streamwise coordinate designated by $x^{m}$, where $m$ refers to the spatial location of each measurement set. An analog was used in the original POD to differentiate snapshots in time as $t^{n}$. Concatenating modes of common rank formulate new sets of snapshots as $\boldsymbol{\Phi}^{(n)}\left(x^{m}\right)$. The difference between the POD and the DPOD two lies in definition of the kernel, which is now a correlation between POD modes at fixed mode number $\boldsymbol{D}^{n}\left(x^{m}\right)$ organized in space, rather than velocity snapshots organized in time:

$$
\boldsymbol{D}^{n}\left(x^{m}\right)=\frac{1}{M} \sum_{m=1}^{M} \boldsymbol{\Phi}^{(n), T}\left(x^{m}\right) \boldsymbol{\Phi}^{(n)}\left(x^{m}\right),
$$

where the index $n$ refers to the POD bases by mode number, the index $m$ now refers to snapshots arranged according to the streamwise coordinate $\boldsymbol{x}$, and $M$ is the number of snapshots (POD modes) used to form the kernel. The DPOD is applied iteratively to sets of $\boldsymbol{D}^{n}\left(x^{m}\right)$, increasing mode number $n$ with each iteration.

The eigenvalue problem is now written,

$$
\boldsymbol{D}^{n} \boldsymbol{B}^{n}=\Lambda^{(n, m)} \boldsymbol{B}^{n}
$$

DPOD modes can be expressed in a form analogous to the previous formulation,

$$
\boldsymbol{\Gamma}^{(n, j)}=\frac{\sum_{j=1}^{M} \boldsymbol{B}^{j}\left(x^{m}\right) \boldsymbol{\Phi}^{(n)}\left(x^{m}\right)}{\left\|\sum_{j=1}^{M} \boldsymbol{B}^{j}\left(x^{m}\right) \boldsymbol{\Phi}^{(n)}\left(x^{m}\right)\right\|}, \quad j=1, \ldots, M .
$$

Streamwise dependence of modes has been removed through the DPOD in the same sense that temporal dependence was removed through the POD. The DPOD modes 
$\Gamma^{(n, j)}$ are the span of $j$ sub-modes of each of the original $n$ POD modes.

As with the snapshot POD, the span of eigenvalues of the DPOD describes the significance of each of the sub-modes in the vectorial space of the POD modes. The eigenvalues $\Lambda^{(n, m)}$ are a matrix with dimensions that match the original number of snapshots $(n)$ at each measurement location and the number of sub-modes $(m$, corresponding to the number of measurement planes).

Analogous to the initial decomposition $\boldsymbol{\Phi}^{(n)}$ may be reconstructed using the submodal structures from the DPOD,

$$
\boldsymbol{\Phi}^{(n)}\left(x^{m}\right)=\sum_{j=1}^{M} b_{n, j} \boldsymbol{\Gamma}^{(n, j)},
$$

where $b_{n, j}$ are the amplitudes of the sub-modes obtained by back-projecting the set of $\Phi$ onto $\Gamma$,

$$
b_{n, j}=\int_{\Omega} \boldsymbol{\Phi}^{(n)}\left(x^{m}\right) \boldsymbol{\Gamma}^{(n, j)} d \Omega .
$$

Amplitudes $b_{n, j}$ communicate the spatial organization and significance of each sub-mode whereas the sub-modes themselves $\Gamma^{(n, j)}$ are independent of $x$. In reconstructing POD modes from sub-modal structures, the dependence of $\Phi^{(n)}$ on $x$ is contained in the sub-modal amplitudes.

In making reconstructions via DPOD, the cross-correlation of POD modes is not the goal, but rather a series of filtered modes. Equation (2.25) can be used to develop $\left.\stackrel{\circ}{\mathbf{\Phi}}^{(n)}\right|^{M_{r}}=\sum_{m=1}^{M_{r}} b_{n, m} \Gamma^{(n, m)}$, where $M_{r}$ is the number of sub-modes in the truncated set. Note that the over-ring is still used to indicate that a quantity has been filtered, even at the sub-modal level. Stresses are then reconstructed with a truncated set of filtered POD modes as, $\left.\stackrel{\circ}{u \nu}\right|_{N_{r}} ^{M_{r}}=\left.\sum_{n=1}^{N_{r}} \lambda^{(n)} \stackrel{\circ}{\phi}_{u}^{(n)} \stackrel{\circ}{\phi}_{v}^{(n)}\right|^{M_{r}}$. The number of sub-modes used 
in filtered POD modes is designated by $M_{r}$.

Double POD as proposed here may be applied to any collection of related POD modes. If there exists no common projection between the elements of the kernel, the DPOD will provide no appreciable organization of sub-modal structures. The method is appropriate for any type of turbulent flow (wake, jet, developing boundary layer, etc.), provided that the POD bases being concatenated for further decomposition are interrelated. Analogous to the snapshot POD over time, the kernel of the DPOD need not be formed of POD modes resulting from measurements at regularly spaced locations. The series of reconstruction coefficients $b_{n, m}$ weights the sub-modes according to their original spatial distribution. If POD modes forming the kernel are regularly spaced, sub-modal amplitudes form uniform series in space. Contrarily, if the kernel is formed from randomly spaced POD modes, $b_{n, m}$ will not yield well-organized spatial dependence of the sub-modes.

The procedure followed in the current work is illustrated in figure 2.2. Each stage of the analysis is designated with a letter from A to E. The figure denotes snapshot sets as long boxes and modes as pegs with heights that vary to convey their relative importance in each modal basis. Horizontal alignment is representative of the organization of snapshots in time. The streamwise coordinate is shown moving back through the figure. The initial application of the POD from A to B results in a basis of 2000 POD modes at each measurement location that are not themselves dependent on time. The temporal dependence of each dataset is accounted for in the series of mode amplitudes $a_{n}$, not shown in the diagram. The independence of $\boldsymbol{\Phi}^{(n)}$ from time is signified by the separation of the basis into elements. Concatenating the POD modes along the streamwise coordinate yields new snapshot sets organized spatially rather than tem- 


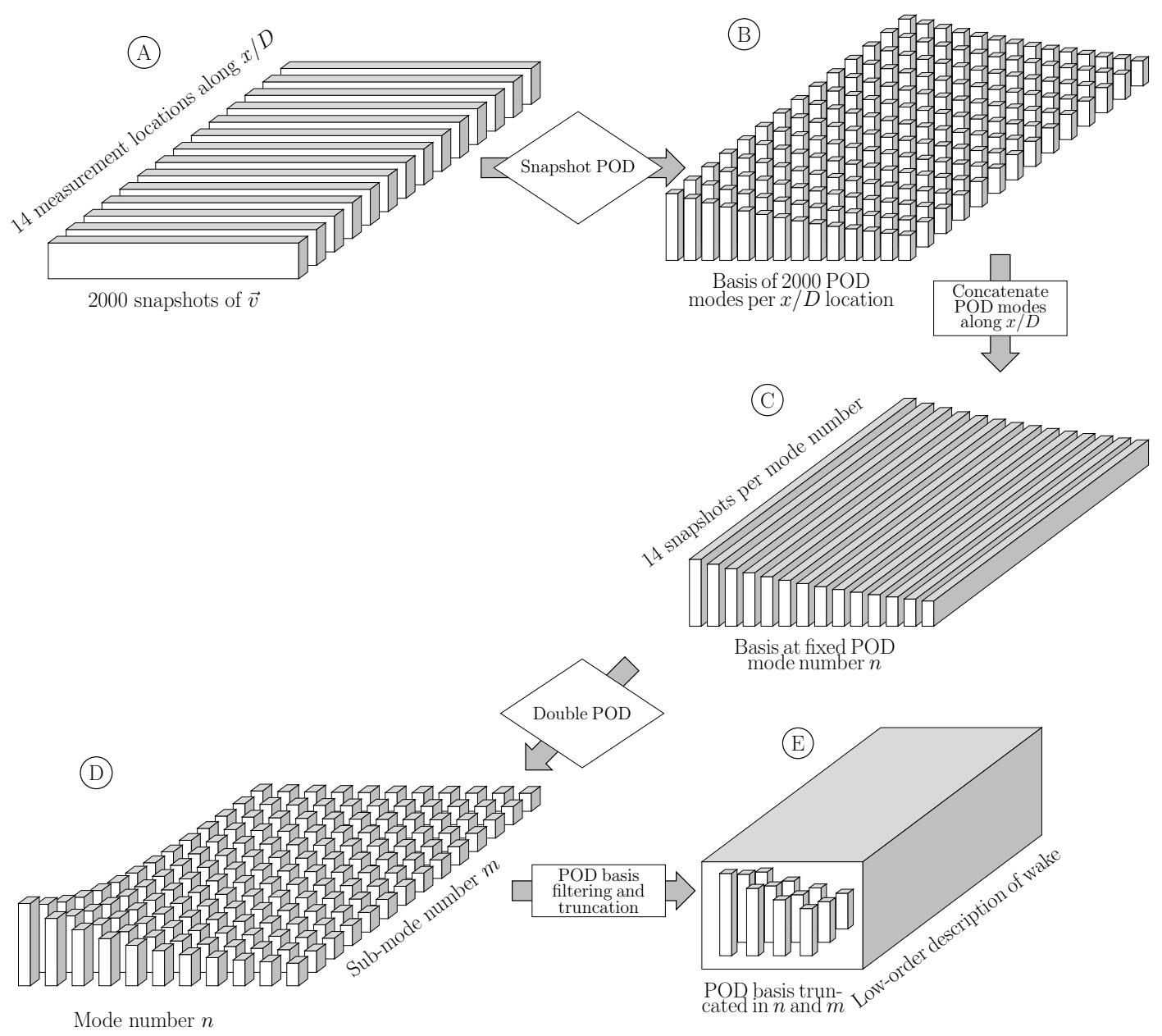

Figure 2.2: Conceptual diagram showing the analysis procedure from velocity snapshots through applications of the POD and formation of a low-order description of the turbulent wind turbine wake.

porally (C).

In a similar sense, the application of the DPOD (from C to D) displaces the dependence of the POD modes on the streamwise coordinate to the sub-modal amplitudes $b_{n, m}$. After secondary decomposition, the separated elements in stage $\mathrm{D}$ are representative of sub-modal structures. The first sub-mode is the largest common projection of fixed POD mode number throughout the wake, an effective average mode immune to 
the switching of the algebraic sign seen in individual POD bases. Truncating the DPOD basis amounts to filtering POD modes at the sub-modal level $\left.\stackrel{\circ}{\mathbf{\Phi}}^{(n)}\right|^{M_{r}}$. Filtered modes are composed by linear combination of the largest common projection (effective average) and successively more nuanced corrections. Limiting the filtered POD basis is the formation of the low-order description of the wake discussed herein. The limited span of filtered POD modes $\left.\stackrel{\circ}{\Phi}^{(n)}\right|^{M_{r}}$ is used to represent the Reynolds stress tensor according to equation (4.19). The selections of thresholds used in truncation are discussed in further detail in the following analysis.

\subsection{Galerkin Projection}

At its foundation, the Galerkin projection seeks an evolution equation of a dynamical system from a subspace of modes or test functions. In the present case, the NavierStokes equations are projected into the Hilbert space formed by the proper orthogonal modes. The Navier-Stokes operator is defined here as,

$$
\mathscr{N}[\boldsymbol{u}]=\frac{\partial \boldsymbol{u}}{\partial t}+\nabla \cdot(\boldsymbol{u} \cdot \boldsymbol{u})-v \Delta \boldsymbol{u}+\nabla P
$$

Terms on the right hand side of equation (2.27) are the transient, convective, viscous, and pressure gradient terms. The velocity field $\boldsymbol{u}$ are considered the trial functions of the Galerkin system. In the Galerkin projection both the test functions and the trial functions are composed of the POD modes, individually or in linear combination as in equation (2.20). The POD basis derived above excludes the mean velocity in the kernel, and is reintroduced as $\boldsymbol{\Phi}^{(0)}$. The respective coefficient $a_{0} \equiv 1$ and is constant in time as the mean velocity is a stationary quantity. The basis functions will be denoted in 
shorthand as,

$$
\boldsymbol{\Phi}=\boldsymbol{\Phi}^{(0)}+\sum_{n=1}^{N} a_{i} \boldsymbol{\Phi}^{(n)} .
$$

Then the Galerkin projection seeks the system,

$$
\frac{d a_{i}}{d t}=f_{i}\left(a_{1}, a_{2}, \ldots, a_{N}\right)
$$

As with the least-squares method undertaken above, the resulting set of coupled ordinary differential equations describe the temporal evolution of each POD mode coefficient. Generally, no exact derivation of the Galerkin system is possible. Rather, a system of $N$ equations are derived from the evolution equation (2.29). The weak form of the Galerkin projection follows,

$$
\|\boldsymbol{v}\| \mathscr{N}[\boldsymbol{u}]=I(\boldsymbol{v}, \boldsymbol{u})+C(\boldsymbol{v}, \boldsymbol{u}, \boldsymbol{u})-v D(\boldsymbol{v}, \boldsymbol{u})+[\boldsymbol{v}, P]_{\partial \Omega}
$$

where $I, C$, and $D$ are projections of the unsteady, convective, and dissipative terms onto the basis of test functions. The last term of equation (2.30) arises from projection of the pressure gradient term and is handled below by solution of the pressure-Poisson equation, square brackets denote surface integration.

Defining the test functions as the POD modes $\boldsymbol{v}=\boldsymbol{\Phi}^{(i)}$, and the trial functions as reconstructed velocity fields according to equation (2.20) $\boldsymbol{u}=\sum_{j=0}^{N} a_{j} \boldsymbol{\Phi}^{(j)}$, the unsteady term from the weak form expands to,

$$
I(\boldsymbol{v}, \boldsymbol{u})=\left\|\boldsymbol{\Phi}^{(i)}\right\| \frac{d}{d t} \sum_{j=0}^{N} a_{j} \boldsymbol{\Phi}^{(j)}=\frac{d a_{i}}{d t}
$$


where Einstein's index notation is employed and summation over the index is implied. The indices $i$ and $j$ are independent, which allows $\boldsymbol{\Phi}^{(i)}$ to be brought into the summation without changing the equality of the statement.

Projection of the dissipation term on the POD basis expands as,

$$
v D(\boldsymbol{v}, \boldsymbol{u})=v\left\|\Phi^{(i)}\right\| \Delta \sum_{j=0}^{N} a_{j} \boldsymbol{\Phi}^{(j)}=v \sum_{j=0}^{N} l_{i j} a_{j}
$$

The coefficient $l_{i j}$ describes the inner product of a mode $i$ and the Laplacian of mode $j$. Similarly, projection of the convection term on the POD basis expands as,

$$
C(\boldsymbol{v}, \boldsymbol{u}, \boldsymbol{u})=\left\|\boldsymbol{\Phi}^{(i)}\right\| \nabla \cdot\left[\sum_{j=0}^{N} a_{j} \boldsymbol{\Phi}^{(j)} \times \sum_{k=0}^{N} a_{k} \boldsymbol{\Phi}^{(k)}\right]=\sum_{j, k=0}^{N} q_{i j k}^{c} a_{j} a_{k}
$$

The pressure gradient term from the Navier-Stokes equations requires a different treatment than the other terms. Instantaneous pressure fields are generally unavailable in experimental data, making pressure gradients notoriously difficult to account for. When fluctuating pressure signals are unavailable, an estimate of the effects of the pressure gradient may be made from the velocity fields through solution of the pressure-Poisson equation.

$$
\Delta P=-\nabla \cdot \nabla \cdot \boldsymbol{u} \cdot \boldsymbol{u}=\sum_{j, k=0}^{N} s_{j k} a_{j} a_{k}
$$

Indices $l$ and $m$ refer to the Cartesian coordinate directions and are used to distinguish 
from mode indices $i, j$, and $k$. Partials source term $s_{j k}$ relate the partial pressures $p_{j k}$ to the POD basis for the velocity field by satisfying the relationship,

$$
\Delta p_{j k}=s_{j k}
$$

along with the Neumann boundary conditions,

$$
\frac{\partial p_{j k}}{\partial x_{n}}=\vec{n} \cdot \nabla p_{j k}=0
$$

Finally, the total pressure field is written,

$$
P=\sum_{j, k=0}^{N} p_{j k} a_{j} a_{k}
$$

The pressure gradient term can now be projected onto the POD expansion as,

$$
\left\|\boldsymbol{\Phi}^{(i)}\right\|-\nabla P=-\left\|\boldsymbol{\Phi}^{(i)}, \sum_{j, k=0}^{N} \nabla p_{j k} a_{j} a_{k}\right\|=\sum_{j, k=0}^{N} q_{i j k}^{p} a_{j} a_{k}
$$

Substituting the parameters derived in equations (2.32), (2.33), and (2.38), for the dissipative, convective, and pressure contributions into the Galerkin system in equation (2.30), it is found that,

$$
\frac{d a_{i}}{d t}=v \sum_{j=0}^{N} l_{i j} a_{j}+\sum_{j, k=0}^{N} q_{i j k}^{c} a_{j} a_{k}+\sum_{j, k=0}^{N} q_{i j k}^{p} a_{j} a_{k} .
$$

The parameters for the convection term and the pressure term are both quadratic and may be combined leading to a simplification $q_{i j k}=q_{i j k}^{c}+q_{i j k}^{p}$. Upon solving the inner product projections above, the series of ordinary differential equations in 
equation (2.39) may be solved numerically, resulting in POD mode coefficients that are functions of time. Initial conditions for each of the ODEs in equation (2.39) are supplied from the POD coefficients. A time series of the velocity field resulting from the Galerkin projection is reproduced according to equation (2.20).

\subsection{Least squares polynomial fit}

An alternate approach to formulating a dynamical system from the POD follows the method of Perret et al. (2006b). There, the dynamical system was proposed following a polynomial expansion of permutations of the POD coefficients. Modes used can be from a POD basis truncated to $N_{r}$ modes and combine as,

$$
\frac{d a_{i}}{d t}=D_{i}+\sum_{j=1}^{N_{r}} L_{i j} a_{j}+\sum_{j, k=1}^{N_{r}} Q_{i j k} a_{j} a_{k}+\sum_{j, k, l=1}^{N_{r}} C_{i j k l} a_{j} a_{k} a_{l}
$$

In equation (2.40), the parameters no longer arise from projecting the governing behavior law onto the POD basis but rather from a least squares fit of monomial terms onto the coefficients directly. The parameters sought are $D_{i}, L_{i j}, Q_{i j k}$, and $C_{i j k l}$, standing for constant, linear, quadratic, and cubic contributions, respectively. Parameters are calculated iteratively for each input mode $i$ and involve many terms. Each index $i, j, k, l$ spans the POD basis selected.

A more consolidated form of the dynamical system is,

$$
\frac{d a_{i}}{d t}=\sum_{k=1}^{N} x_{k} A_{k}\left(a_{1}, \ldots, a_{N_{r}}\right)
$$

where $x_{k}$ are the unknown parameters and $A_{k}$ are the monomial terms at most cubic. There are a total of $N_{p}$ parameters that couple the mode coefficients, found by 
minimizing the error function,

$$
\chi^{2}=\sum_{p=1}^{N_{s}}\left[\frac{d a_{i}}{d t}-\sum_{k=1}^{N} x_{k} A_{k}\left(a_{1}\left(t_{p}\right), \ldots, a_{N_{r}}\left(t_{p}\right)\right)\right]^{2}
$$

Minimization is accomplished numerically by $\chi^{2}=|A X-B|^{2}$, where $X$ is the vector of unknown coefficients, $B$ the vector containing the $N_{s}$ samples of $d a_{i} / d t$ and $A$ the matrix of terms $a_{j}\left(t_{p}\right), a_{j}\left(t_{p}\right) a_{k}\left(t_{p}\right)$, and $a_{j}\left(t_{p}\right) a_{k}\left(t_{p}\right) a_{l}\left(t_{p}\right)$. In the definition of the coupled set of ordinary differential equations (ODEs) in equation (2.40), the POD modes themselves are not employed in the calculating the behavior of the system. One main advantage is that this approach includes cubic terms which are known to add complexity to the system without introducing non-negligible instability Perret et al. (2006b); Braud et al. (2004); Favier et al. (2008).

A potential detriment to large mode bases is that the system requires that a large number of parameters be calculated. The total number of parameters calculated is then $N_{p}=N_{r}+N_{r}^{2}+N_{r}^{3}+N_{r}^{4}$, for the constant, linear, quadratic, and cubic terms coupling each mode to every other mode in the basis. The system requires that there be records of both $a_{i}$ and $d a_{i} / d t$. This constraint is not insurmountable with direct numerical and large eddy simulations, but poses a challenge for many experimental approaches.

Numerical solution of the ODEs in equation (2.40) results in a new time series of coefficients predicting the relative intensities of each of the modes forming the LODS. Any quantity issuing from the POD dynamical system will be denoted below with a caret $\left(^{\wedge}\right)$. Thus the least-squares fit of the time derivatives of the POD coefficients is written $d \hat{a}_{i} / d t$, the mode coefficients from the LODS are $\hat{a}_{i}$, and are a function of a 
new vector of time values $\hat{t}$. The time resolution of $\hat{a}_{i}(\hat{t})$ is related to the error tolerance of the numerical ODE solver and the number of modes in the system Perret et al. (2006b); D’adamo et al. (2007). 


\section{Chapter 3}

\section{Experimental setup}

\subsection{Wind tunnel Experiment}

An array of wind turbines was made at model scale in the closed circuit wind tunnel facility at Portland State University. The wind tunnel features a 9:1 contraction ratio to eliminate the effects of noise introduced by the powering fan and guide vanes in the corners. The cross section of the wind tunnel is constant with dimensions of 1.2 $\mathrm{m} \times 0.8 \mathrm{~m}$ (spanwise and vertical directions, respectively) throughout the length of the test section. The inlet of the test section was furnished with a passive grid composed of seven horizontal and six vertical rods to introduce large scale turbulence to the flow. Vertical strakes (0.0125 m thick acrylic) shaped to precondition the boundary layer in the wind tunnel in order to increase the high-shear region of the flow near the wall. The strakes were spaced by $0.136 \mathrm{~m}$ across the width of the tunnel, $0.35 \mathrm{~m}$ downstream of the passive grid. Roughness was added to the inlet of the flow in the form of small-diameter chains. All surfaces of the test section have been constructed of Schlieren-grade annealed float glass mounted to the framework of the tunnel to enable non-intrusive optical measurement techniques in multiple configurations. Further detail on the wind tunnel and the setup for wind turbine array experiments, including a 
characterization of the inflow to the model-scale wind farm, is found in Hamilton et al. (2015a).

The model-scale wind turbines (Figure 3.1) were fabricated in-house, designed to emulate a $1 \mathrm{MW}$ horizontal-axis device commonly used in full scale wind energy projects. Model turbines have three blades with a pitch at the base of approximately $\gamma_{\text {root }}=22^{\circ}$ and at the tip of approximately $\gamma_{\text {tip }}=15^{\circ}$. The $7^{\circ}$ twist from root to tip approximates the aerodynamics of wind turbine blades at the laboratory scale. The nacelle of each model turbine is composed of an electric motor (Faulhaber GMBH \& Co., Series 1331T012SR). The motors are powered at the shaft, and behave as generators producing current and a voltage difference across the motor leads, through which the operating point of each motor is controlled by the application of resistive elements. Each model turbine was tuned to match the peak power coefficient according to studies made with the same models and wind tunnel Hamilton et al. (2015a,b); Hamilton and Cal (2015). (Measurements of power and power coefficient are not discussed in the present work but can be found in Hamilton and Cal (2015).) The hub height of each turbine model was equal to the rotor diameter $D$.

Recent wind tunnel experiments consider a Cartesian array to be fully developed after the fourth streamwise row of devices; beyond the fourth row of model turbines, the statistics become approximately periodic from row-to-row Chamorro et al. (2011); Chamorro and Porté-Agel (2011b). For the present experiment, the wind turbines were arrayed to match established wind tunnel arrangements to aid in comparison with previous results Hamilton et al. (2012, 2015a,b); Hamilton and Cal (2015). Figure 3.2 depicts geometry of the wind turbines in the model array relative to measurement planes. The spacing of model turbines was $6 D \times 3 D$ in the streamwise and spanwise directions, 


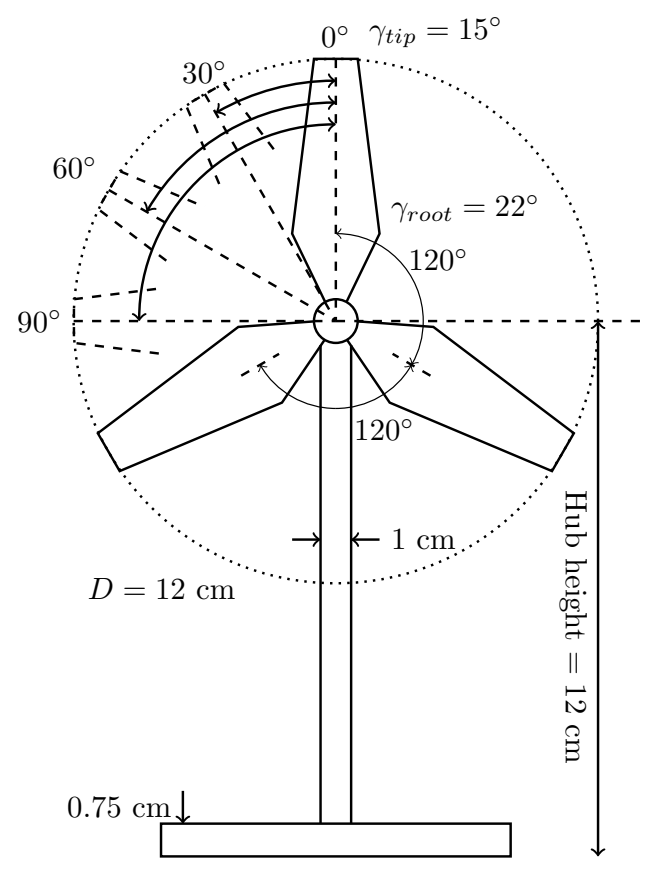

(A)

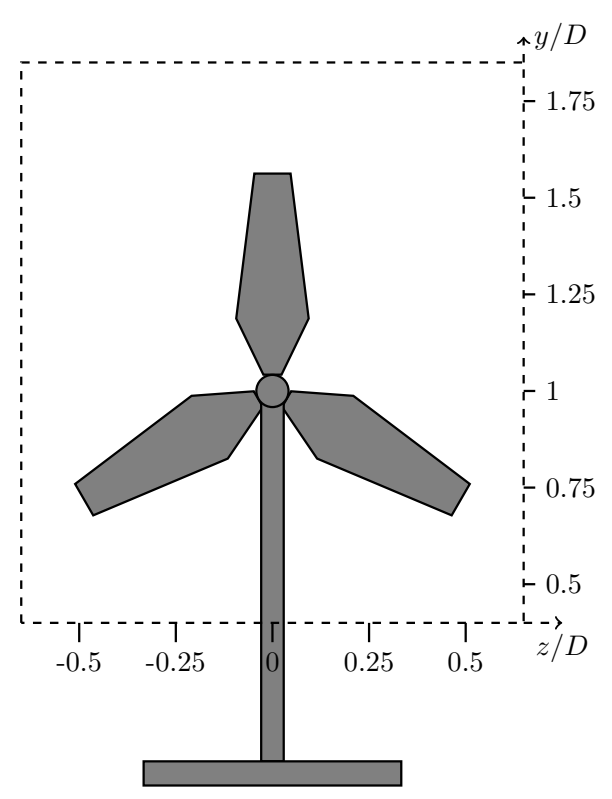

(B)

Figure 3.1: Schematic of the model-scale wind turbines used in the wind turbine array, figure 3.1(A). Model is also shown relative to the SPIV measurement window in the wakes with affixed coordinate system, figure 3.1(B).

respectively (denoted as $S_{x}$ and $S_{z}$ ). Spacing of the turbines is expected to amplify wake interaction and development of the wind turbine canopy layer.

Velocity measurements were undertaken with stereo particle image velocimetry (SPIV) in planes parallel to the swept area of the rotor. Figure 3.2 relates the measurement fields to the model array used in the wind tunnel. SPIV enables two dimensional, three component (2D-3C) measurements, in each plane accessing the full Reynolds stress tensor and in-plane gradients. Measurement planes in the current experiment are oriented to resolve gradients across the wakes. A common scaling argument asserts that gradients along the streamwise coordinate are expected to be smaller than 
those in the wall-normal and spanwise directions except immediately following the wind turbine $(x / D \leq 1)$. Each SPIV measurement window is approximately $0.2 \mathrm{~m} \times$ $0.25 \mathrm{~m}$ in dimension. Snapshots were taken at a time delay of $\delta t=180 \mu \mathrm{s}$. The Nd:Yag (532 nm, $1200 \mathrm{~mJ}, 4 \mathrm{~ns}$ duration) double-pulsed laser sheet varied from $1 \mathrm{~mm}$ at the bottom of the tunnel to approximately $2 \mathrm{~mm}$ at the top of the measurement window.

The time delay and sheet thickness were tuned to minimize the out of plane loss of particle pairs while maximizing light intensity. The flow was periodically reseeded with vaporized di-ethyl-hexyl-sebacate and the concentration of the seeding was kept at a constant level to ensure consistency of particle imaging. Cameras (4MP ImagerProX) used to collect flow snapshots were arranged beneath the wind tunnel floor looking vertically upward in the general direction of the laser sheet, placing them in backscatter. Cameras were approximately $45 \mathrm{~cm}$ apart arranged symmetrically around the streamwise coordinate. Velocity vectors were calculated using a multi-pass FFT based algorithm with two passes each at 64 and 32 pixel interrogation windows. The final spatial resolution of velocity vectors was approximately $1.4 \mathrm{~mm}$ in both the spanwise and wall-normal directions. Each streawmise location required an individual calibration to ensure accurate results. Each measurement set along $x / D$ contains 2000 SPIV snapshots for converged statistics. Statistical error of second order quantities was calculated to be approximately $3 \%$. Measurement error was calculated following the statistical variability methods outlined by George (2009).

The position of the rotor blade was located with a Monarch remote optical sensor placed outside the wind tunnel and able to see a small square of reflective tape affixed to one blade of the rotor. With each pass of the tape the sensor initiated a square wave signal that in turn triggered the SPIV system. Four positions of the rotor were consid- 


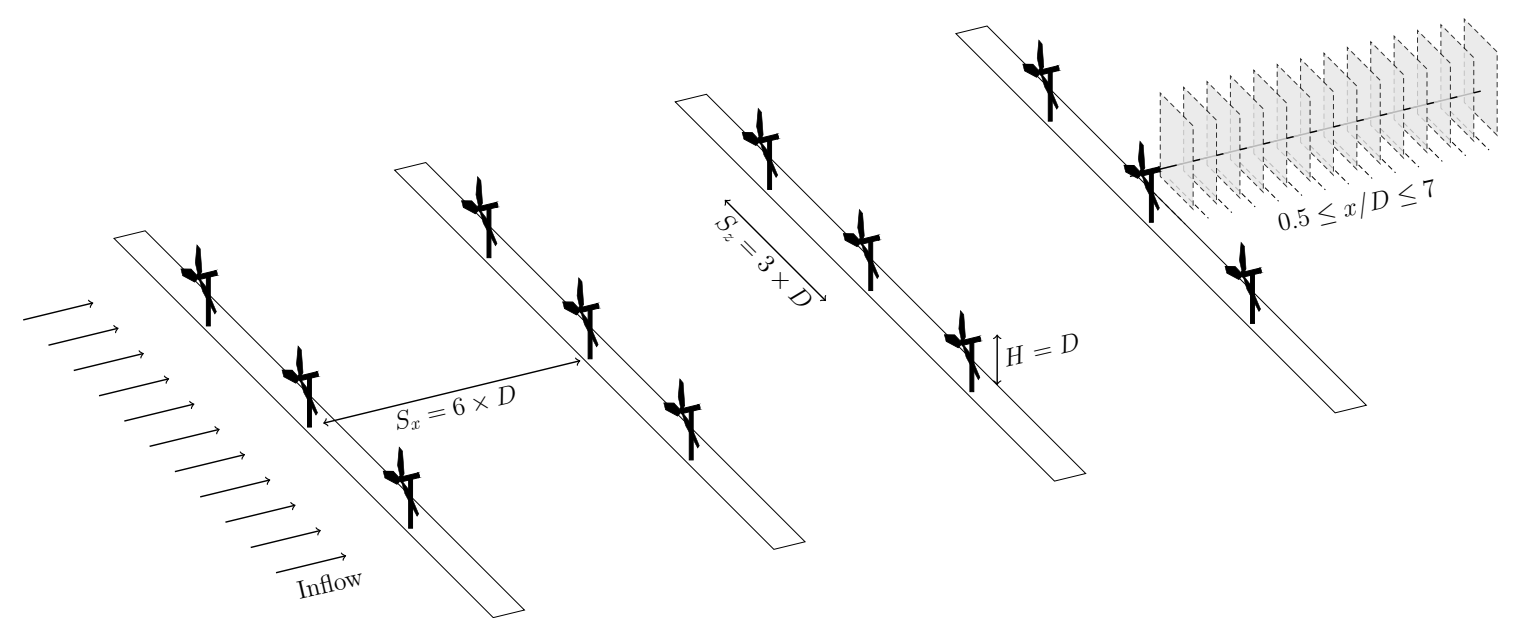

Figure 3.2: Model-scale array used in the current experiment relating turbine spacing and measurement locations behind the fourth row.

ered in the experiment, $\alpha \in\left[0^{\circ}, 30^{\circ}, 60^{\circ}, 90^{\circ}\right]$, where $0^{\circ}$ was one blade oriented vertically upward. Each SPIV measurement window is approximately $0.2 \mathrm{~m} \times 0.2 \mathrm{~m}$ in dimension. Snapshot pairs were taken at a time delay of $\delta t=180 \mu \mathrm{s}$. The Nd:Yag (532 nm, $1200 \mathrm{~mJ}, 4$ ns duration) double-pulsed laser sheet varied from $1 \mathrm{~mm}$ at the bottom of the tunnel to approximately $2 \mathrm{~mm}$ at the top of the measurement window.

\subsection{Turbulent channel flow - direct numerical simulation}

Development of the POD and anisotropy invariant analysis techniques is explored with the wind turbine wake data collected in the experiment described above as well as a direct numerical simulation (DNS) of a fully-developed channel flow simulated at Johns Hopkins University (JHU). A brief overview of the direct numerical simulation (DNS) of a fully-developed channel flow simulated at Johns Hopkins University (JHU) is provided here with particular attention payed to the sample data extracted from the simulation. For a complete description of the procedure and simulation, the reader 
is referred to the documentation provided by JHU and summarized in Graham et al. (2013) (see also, Li et al. (2008); Perlman et al. (2007)). Here, focus is placed on interpretation the POD and reduced order models. Accordingly, a relatively small sample of the full channel flow is selected, limiting both the spatial and temporal ranges of the data.

The channel flow is one of several direct numerical simulations conduced at Johns Hopkins University and made available for wide use. The DNS of the the fullydeveloped channel flow uses periodic boundary conditions in both the streamwise and spanwise directions and no-slip boundary conditions at the walls. The Navier-Stokes equations are solved using a wall-normal velocity-vorticity formulation (Kim et al., 1987), according to which solutions are provided using a Fourier-Galerkin pseudospectral method for the streamwise and spanwise directions and seventh-order Basissplines (B-splines) collocation method in the wall normal direction. Time integration is performed using a third-order Runge-Kutta method. The simulation was performed using the petascale DNS channel flow code (PoongBack) (Lee et al., 2013). The simulation is performed for approximately a single flow through time of 26 non-dimensional seconds.

Data sampled from the full DNS and discussed below represent a small subset of the total provided in the database. The location of the sample volume was selected at random in both the $x$ - and $z$-coordinates but fixed for all samples. The near-wall region is of particular interest for the current study as it is represents a challenge for reduced-order models. Data spans the channel half-height, $-1 \leq y / H \leq 0$ (normalized by the channel half-height $H$ ). In viscous units, data spans $0 \leq y^{+} \leq 1000$, renormalized by the viscous length scale $\delta_{v}=1 \times 10^{-3}$. Resolution of the data in the streamwise di- 
rection is set to $\Delta x / H=0.0061$ and in the spanwise direction to $\Delta z / H=0.0123$, again normalized by the channel half-height. A total of 550 snapshots were sampled from the channel flow, representing a small portion of the full simulation time, $t \in[0,3.57]$. Parameters of the sampled data are summarized in Table 3.1, and visualized in the schematic in Figure 3.3. Turbulence seen in the central region of the channel is expected to exhibit the passage of large, anisotropic structures, although in an ensemble sense, the turbulence in the outer layers is more isotropic. The half-channel velocity profile is shown in viscous units in Figure 3.4.

Table 3.1: Details of sampled volume data.

$\begin{array}{lcc}\text { spacing in } x \text {-direction } & \Delta x & 0.0061 \\ \text { spacing in } y \text {-direction } & \Delta y & \text { varies with wall-normal distance } \\ \text { spacing in } z \text {-direction } & \Delta z & 0.0123 \\ \text { time resolution between snapshots } & \Delta t & 0.0065 \\ \text { measurement points } & n_{x} \times n_{y} \times n_{z} & 32 \times 64 \times 24 \\ \text { number of snapshots } & n_{t} & 550\end{array}$

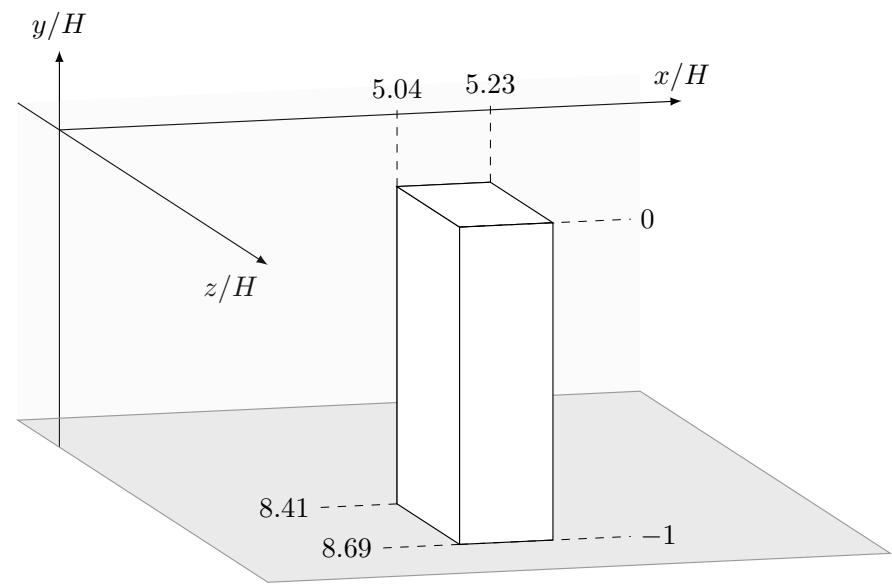

Figure 3.3: Schematic of the lower half of the channel flow DNS simulation space. Only a small region of the total channel is shown. Sampling window (white rectangle) was sized to span the inner layers. Location of the window in $x / H$ and $z / H$ was selected randomly. 


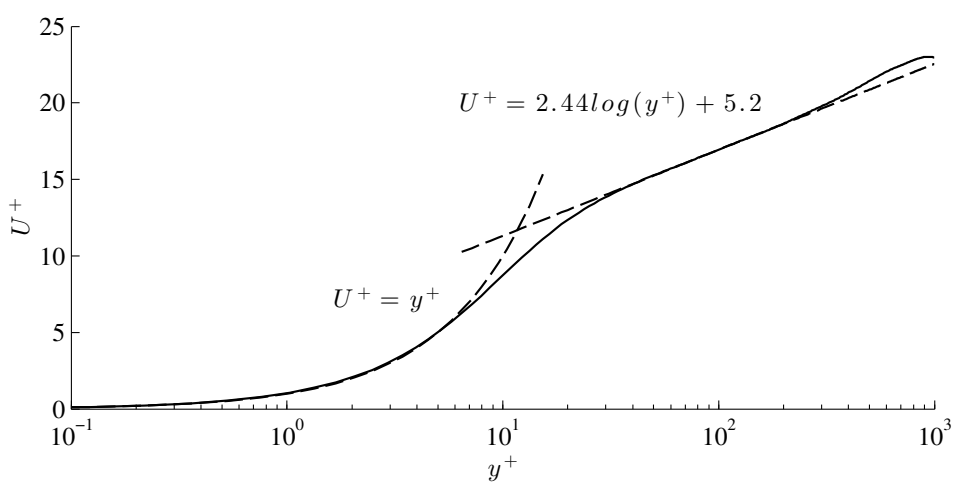

Figure 3.4: Half-channel velocity profile. Dashed lines correspond to the viscous sublayer and the log-layer.

\subsection{Large eddy simulations of wind farms}

The low-order dynamical system proposed in equation (2.40) requires simultaneous estimates of both the POD coefficients $a_{n}$ and their respective time derivatives $d a_{n} / d t$. Estimates for the time derivate naturally depend on the time resolution of the measurements input to the POD algorithm. The recording frequency of the SPIV system at Portland state has a nominal speed of $5 \mathrm{~Hz}$, far slower than would be necessary to make accurate estimates of the model-scaled wind turbines used in the experiments described above. In previous work with LODS (Perret et al., 2006b), a technique known as dual-time PIV was employed to get measurements sufficiently close in time that accurate derivatives could be calculated. Because these sorts of measurements are not currently possible at PSU, the dynamical systems derived below rely on wind turbine and array data issuing from a series of large eddy simulations (LES) conducted at the University of Utah. The simulation scheme is briefly described below. For greater detail on the simulations the reader is referred to Calaf et al. (2010); Sharma et al. (2015).

The data used in the following work relies on the LES code introduced in Sharma 
et al. (2015), which integrates the non-dimensional, incompressible, and filtered Navier-Stokes momentum equations together with the continuity equation. The equations are implemented using a rotational form to assure conservation of energy and mass of the inertial terms (Orszag and Pao, 1974). The effects of temperature were removed from the simulation, decoupling the temperature and velocity fields and forcing neutral atmospheric stratification. The flow is forced by a pressure boundary condition in order to ensure the flow remains perpendicular to the wind turbine rotor. These simplifications were imposed in order to match the experimental data described above as closely as possible.

$$
\begin{gathered}
\frac{\partial \tilde{u}_{i}}{\partial x_{i}}=0 \\
\frac{\partial \tilde{u}_{i}}{\partial t}+\tilde{u}_{j}\left(\frac{\partial \tilde{u}_{i}}{\partial x_{j}}-\frac{\partial \tilde{u}_{j}}{\partial x_{i}}\right)=-\frac{1}{\rho} \frac{\partial p^{*}}{\partial x_{i}}-\frac{\partial \tilde{\tau}_{i j}}{\partial x_{j}}+f_{i},
\end{gathered}
$$

In equations (3.1) and (3.2), a tilde ( ) indicates an instantaneous quantity resolved above the LES filtering operation at the grid-size $\Delta$.The term $\tilde{\tau}_{i j}$ term represents the deviatoric part of the momentum sub-grid stress (SGS) term, $\tilde{\tau}_{i j}$ which is modeled using the Lagrangian Scale Dependent model of Bou-Zeid et al. (2005). The filtered pressure term has been modified $\left(p^{*}\right)$ to include the trace of the SGS tensor $\left(\tilde{p} / \rho+\tilde{\tau}_{k k} / 3\right)$. As in the development presented in equation (2.2), a forcing term $f_{i}$ is necessary to represent the momentum sink induced by the wind turbine that includes both an axial and tangential component. In the simulation the body force per unit volume $f_{i}$ is modeled using the actuator-disk with rotation (ADR, see Wu and Porté-Agel (2011)).

Common practice in LES of atmospheric flows, viscous effects are considered neg- 
ligible as the flow is at very large Reynolds number. Numerical discretization of the equations follows that introduced by Albertson and Parlange (1999); Moeng (1984), wherein a pseudo-spectral approach with a staggered-grid is used. Fourier discretization is employed in the streamwise and spanwise directions making the periodic and eliminating the need for lateral boundary conditions. The top boundary has zero-flux and zero-shear boundary conditions such that the vertical velocity and the gradients of the horizontal velocities equal to zero. At the lower surface has a no-slip condition and, because of the staggered grid, an equivalent shear stress is imposed at the first grid point for the horizontal velocities parameterized from the traditional log-law.

The LES analyzed here models a single wind turbine in the atmospheric boundary layer, equivalent to a wind farm where the constituent wind turbines are highly spaced. Given the size of the numerical domain, the wake of the wind turbine will not interact with itself, regardless of periodic boundary conditions. The simulation domain consists of $n_{x}=512, n_{y}=129, n_{z}=644$ nodes in the streamwise, wall-normal, and spanwise directions, respectively. In physical dimensions, the simulation space occupies $L_{x}=12.3, L_{y}=1$, and $L_{z}=1.5$ kilometers. Simulation domain dimensions are shown in the schematic in Figure 3.5. With periodic boundary conditions, the simulation is equivalent to a series of turbines spaced by approximately $S_{x}=120 \mathrm{D}$ and $S_{z}=30 \mathrm{D}$ in the streamwise and spanwise directions.

The following analysis focuses on the wake of the wind turbine in the LES data; a subdomain of the simulation is extracted detailing the wake only. Figure 3.5 shows the wake subdomain as a volume inside the full LES domain. A low-order dynamical system is developed below characterizing turbulent fluid dynamics following for the wake only. It is understood from previous work (Hamilton et al., submitted for pub- 


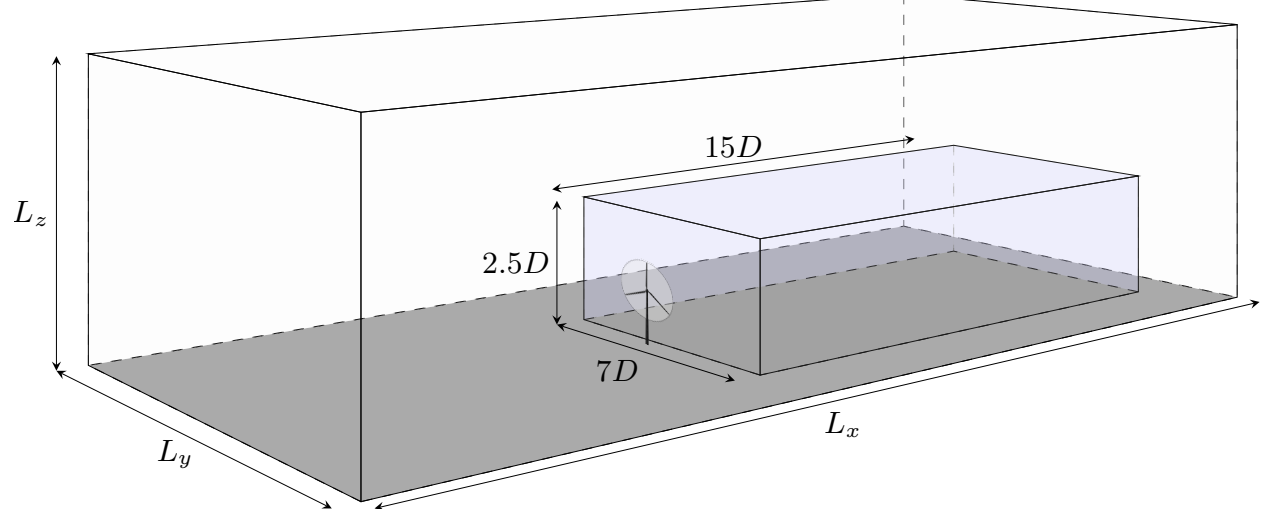

Figure 3.5: Simulation domain for wind turbine LES. Inner volume represents the subdomain analyzed in the present work. The light gray circle indicates the swept area of the rotor over which velocity is integrated to calculate $U_{\text {eff }}$.

lication May, 2016) that coefficients predicted by the low order dynamical system will diverge from those of the POD in long solution times. To keep error associated with the modeled turbulence field within tolerances, the dynamical system is periodically halted and recalibrated with new initial conditions supplied through open-loop transfer functions relating the effective inflow velocity to the expected coefficient values. Effective inflow velocity is calculated by integrating the instantaneous streamwise velocity over the swept area of the rotor immediately upstream of the turbine, shown as a gray circle in Figure 3.5. The effective inflow velocity is calculated as,

$$
U_{\text {eff }}=\int_{A_{\text {rotor }}} \tilde{u}(t) d A .
$$




\section{Chapter 4}

\section{Results}

\subsection{Phase-locked wind turbine wake statistics}

Velocities and stresses have been normalized by the time-averaged inflow velocity at hub height of the model wind turbine, $U_{h u b}=4.75 \mathrm{~ms}^{-1}$. The momentum deficit of the wake of the wind turbine is visible in the contours of the mean streamwise (axial) velocity $U$ shown in figure 4.63(A). The minimum value of $U / U_{h u b}=0.1$ occurs directly following the nacelle of the model wind turbine at $x / D=0.5$. In the conditions of this experiment, it is not uncommon to see instantaneous recirculations at $x / D=0.5$. However, over a large set of samples any negative streamwise velocities are washed out by ensemble averaging.

In agreement with previous wind turbine array experiments, the component of highest magnitude in the Reynolds stress tensor is the streamwise normal stress $\overline{u^{2}}$. Shown in figure 4.10(B), the streamwise normal stress forms a crescent-shaped struc-

The results in the current section are developed from a set of phase-locked SPIV measurements taken in the Portland State University wind tunnel. They have been the subject of several conference presentations and will appear as a book chapter in the forthcoming volume, Hamilton, N., M. Tutukn, and R. B. Cal. "Turbulent and deterministic stresses in the near wake of a wind turbine array." Invited contribution to Whither Turbulence and Big Data in the 21st Century.' Springer Volume. (release forthcoming). 


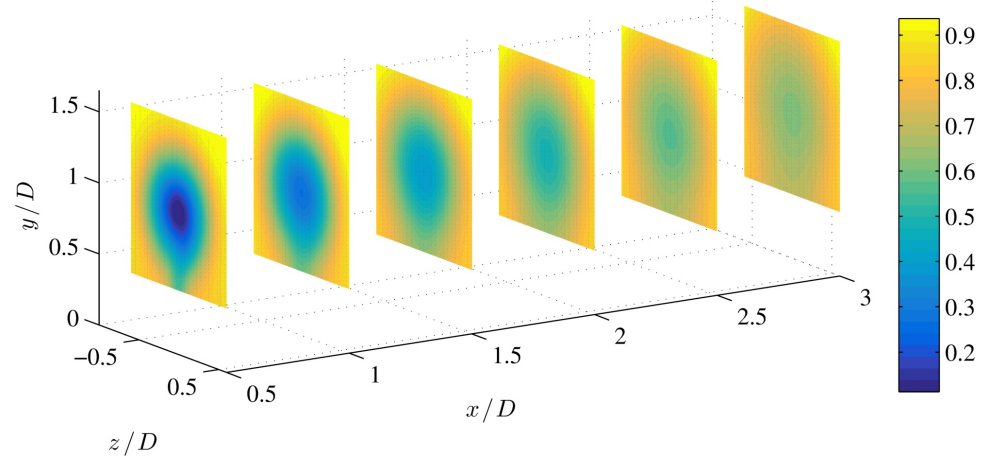

Figure 4.1: Time averaged streamwise velocity, $U / U_{h u b}$. The contours above show the mean velocity averaged over all measurements and are independent of phase.

ture in time-averaged contour plots. Specific evidence of the passage of the rotor is only seen very close to the model wind turbine, at $x / D=0.5$. Beyond that point, features evidencing the passage of the rotors are washed out by mixing and distortions of the flow from large turbulence structures. Peak values of $\overline{u^{2}}$ occur approximately $x / D=1.5$ downstream from the wind turbine rotor similar to previous studies of wind turbine wakes (Hamilton et al., 2012, 2015a). The streamwise normal stress in figure 4.10 (B) is on the order of unity when normalized by the square of the hub height velocity. Wall-normal and spanwise normal stresses (not shown for brevity) are less than half the magnitude of $\overline{u^{2}} / U_{h u b}^{2}$. Contributions to the overall flux of kinetic energy by $\overline{u^{2}} / U_{h u b}^{2}$ are negligible due to small gradients in the streamwise direction. Timeaveraged shear stresses are included in figure 4.2 for comparison to phase-dependent contributions below. The shear stresses are roughly antisymmetric in the vertical direction for $-\overline{u v} / U_{h u b}^{2}$ in figure 4.2(B) and horizontally for $-\overline{u w} / U_{h u b}^{2}$ in figure $4.2(\mathrm{C})$.

There are two quantities contributing to the resupply of kinetic energy through the flux term in equation (2.2). The shear stresses $-\langle u v\rangle_{\alpha} / U_{h u b}^{2}$ and $-\langle u w\rangle_{\alpha} / U_{h u b}^{2}$ (figure 4.65(A) and 4.65(B), respectively) contribute to the vertical and lateral entrain- 


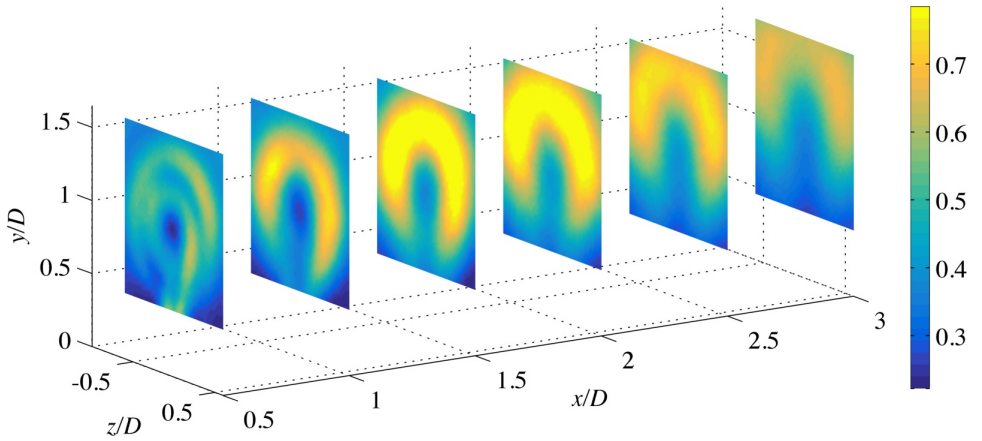

(A) $\overline{u^{2}} / U_{h u b}^{2}$

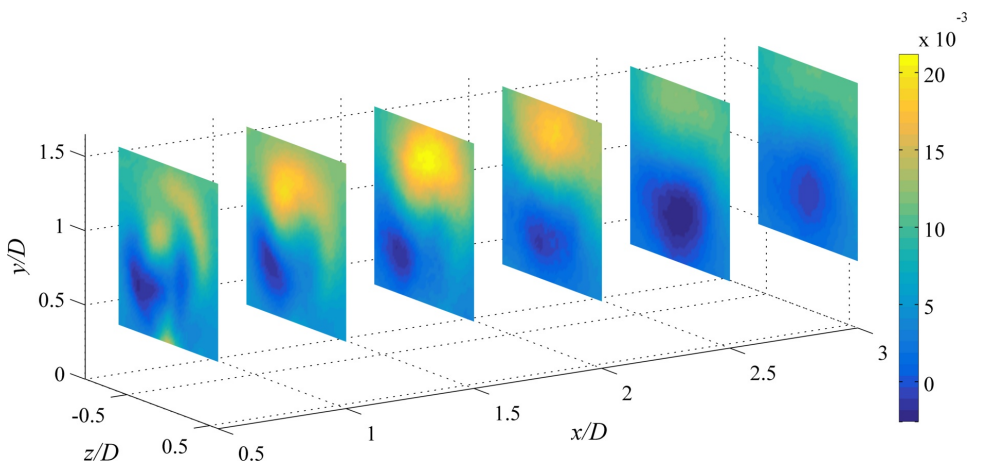

(B) $-\overline{u v} / U_{h u b}^{2}$

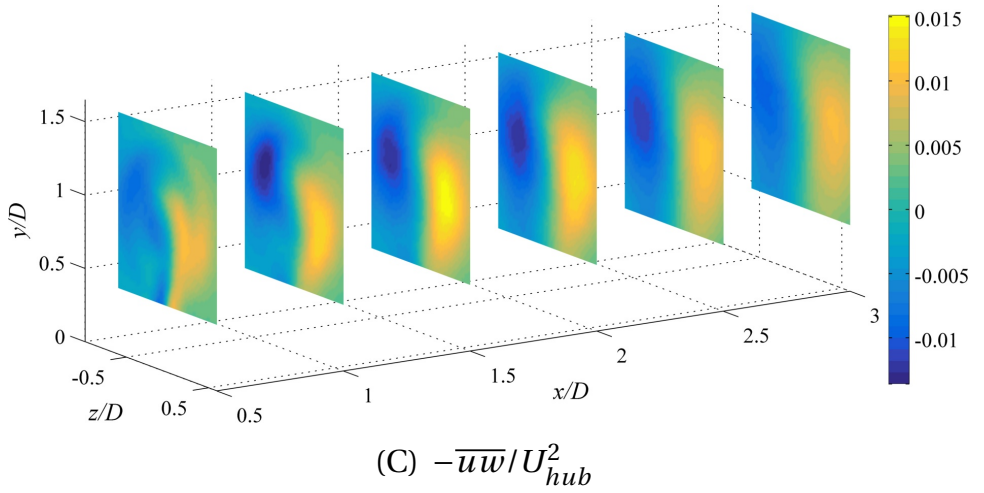

Figure 4.2: Time averaged Reynolds stresses; independent of phase.

ment of high momentum flow from outside the wake. Second-order quantities reflect the growth of the wake shown in contours of the mean velocity in figure $4.63(\mathrm{~A})$. The phase-averaged shear stresses show that the contributions to the stress field are prone 
to periodic peaks, most evident for $x / D \leq 2$.
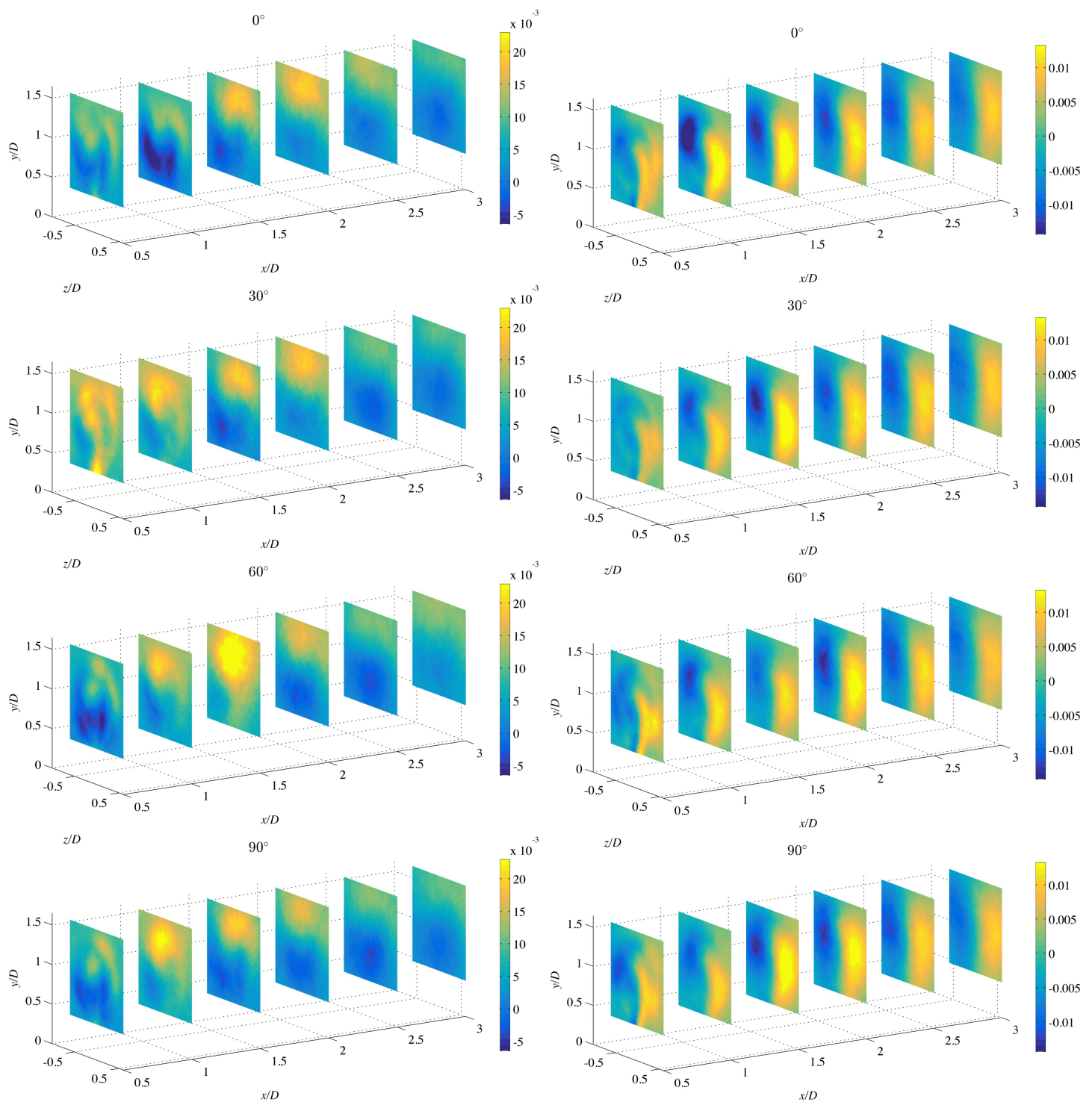

$$
\text { (A) }-\langle u v\rangle_{\alpha} / U_{h u b}^{2}
$$

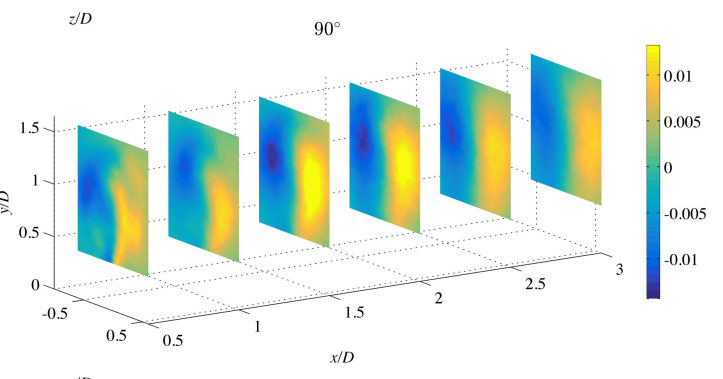

(B) $-\langle u w\rangle_{\alpha} / U_{h u b}^{2}$

Figure 4.3: Phase-averaged Reynolds stresses in the near wake.

The Reynolds shear stress composed of the streamwise and wall-normal fluctuations is discussed as its contribution to the flux and production terms of the kinetic 
energy budget is the most significant (see e.g. Cal et al. (2010); Hamilton et al. (2012)). Figure 4.65(A) shows that $-\overline{u v}$ is positive for most locations of the near wake above hub height, with the exception being directly behind the mast of the wind turbine. The contours of $-\langle u v\rangle_{\alpha=30^{\circ}}$ and $-\langle u v\rangle_{\alpha=60^{\circ}}$ show regions in the near wake where the flux is of greater magnitude than the $-\overline{u v}$ because the position of the rotor allows the flow to be advected into the wake. The region where $-\langle u v\rangle_{\alpha=0^{\circ}}<0$ is similarly more extreme than the respective region of $-\overline{u v}$. As the turbine blades rotate around the hub, the gap between individual blades is sufficient for turbulence structures to pass through undisturbed. In other regions of the flow, the blades disturb the direct advection of large structures into the wake; rather they are effectively cut into smaller structures by the blades.

In ideal conditions (uniform inflow of low turbulence intensity), tip vortices develop from the rotor and are convected downstream, illustrated by Hu et al. (2012). The current experiment places model wind turbines in an array and the inflow to the wind turbine investigated here is accordingly turbulent; inflow turbulence intensity to the model wind turbine is approximately $20 \%$ (not shown). Periodicity in the flow is further complicated by the addition of the boundary layer in the wind turbine array. Accordingly, rotation of the flow is distorted and becomes difficult to visualize, especially by ensemble averaging. However, the phase-averaged vertical flux of kinetic energy, shown in figure 4.4, demonstrates variation between phase orientations of the rotor, indicating that there are periodic contributions to the entrainment process. Typically this quantity is discussed as bringing high-momentum flow into the wake from above the turbine canopy. The general trend of the flux of kinetic energy is into the wake from outside which may include lateral transport. Below the nacelle of the wind 

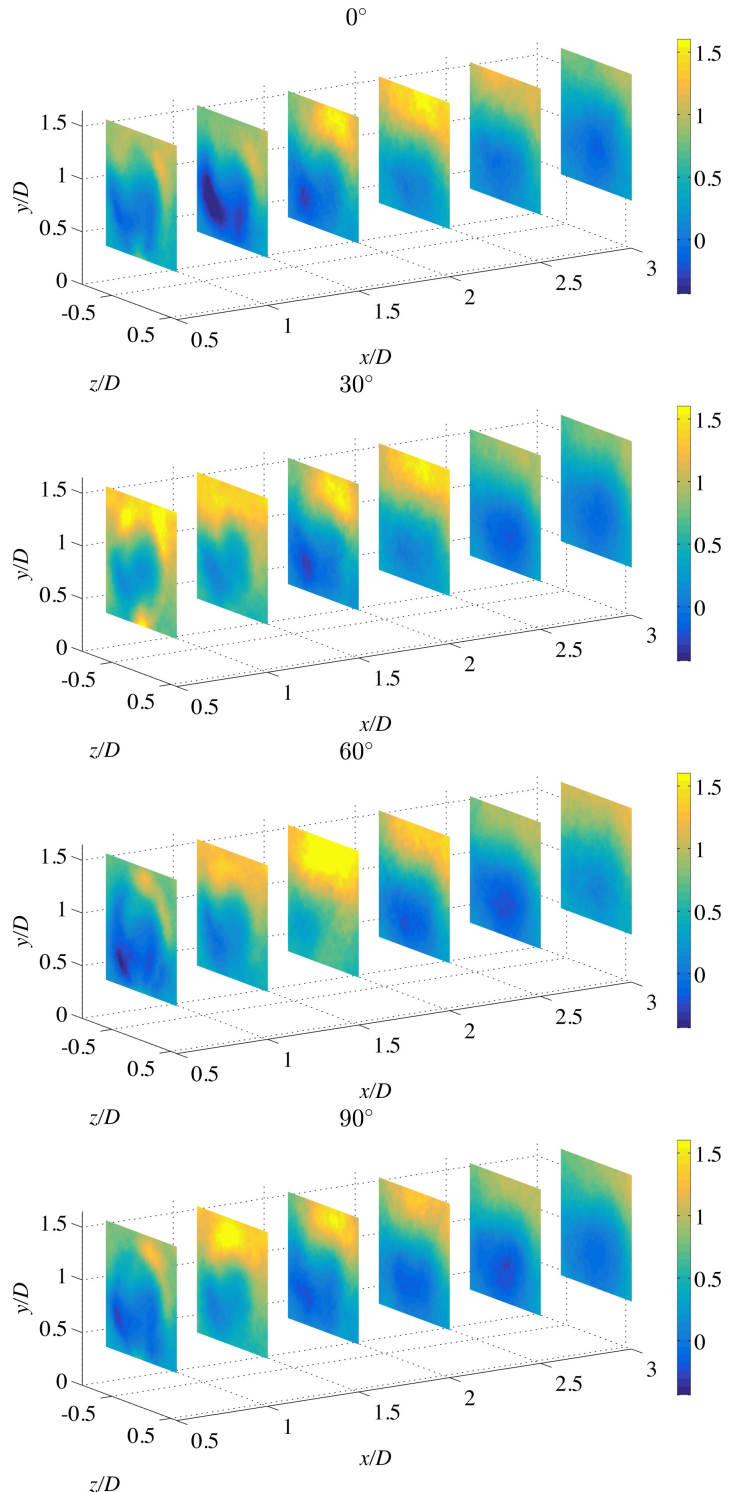

Figure 4.4: Phase-averaged flux of kinetic energy $\langle u v U\rangle_{\alpha}$.

turbine the flux of kinetic energy is away from the center of the wake, most clearly for $-\langle u v U\rangle_{\alpha=30^{\circ}}$ at $x / D \leq 1$ and $-\langle u v U\rangle_{\alpha=60^{\circ}}$ at $x / D=1.5$.

A further consideration for the flux of kinetic energy is the contribution by the deterministic stresses from equation (2.6), shown in figure 4.5. Although the turbulent 
stresses show significant dependence on the orientation of the rotors, the deterministic stresses are approximately two orders of magnitude smaller. The deterministic shear stress $-\overline{u^{\prime \prime} v^{\prime \prime}}$ exhibits azimuthal periodicity ( $\theta$ from the schematic in figure 3.1) around the rotor diameter. The contours in figure 4.5 represent the wave-like contribution added to the mean flow field. They are independent of phase orientation of the rotor blades and can be viewed as the underlying periodicity in the wake.

The flux composed with only the streamwise/wall-normal deterministic stress is shown in figure 4.6. The magnitude of this contribution, like the stresses above, is approximately two orders of magnitude smaller than the phase-dependent or time averaged quantities. The magnitudes of the flux composed with the deterministic stresses indicates that the periodic contributions to the total behavior can be neglected at the first order.
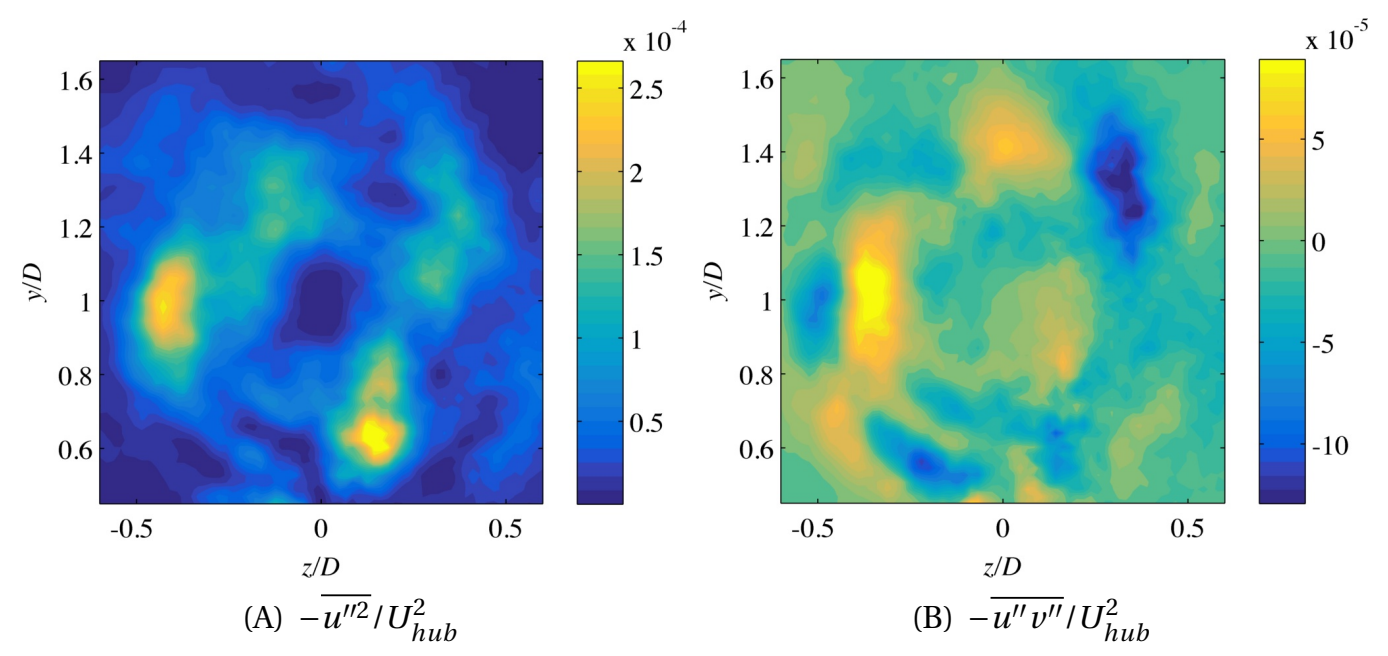

Figure 4.5: Normal and shear deterministic stresses composed of ensemble averaged deviations between phase-averaged and total mean velocities at $x / D=0.5$.

That the deterministic stresses are orders of magnitude less than the phase- and time-averaged contributions is not unexpected given the nature of the turbulent flow 


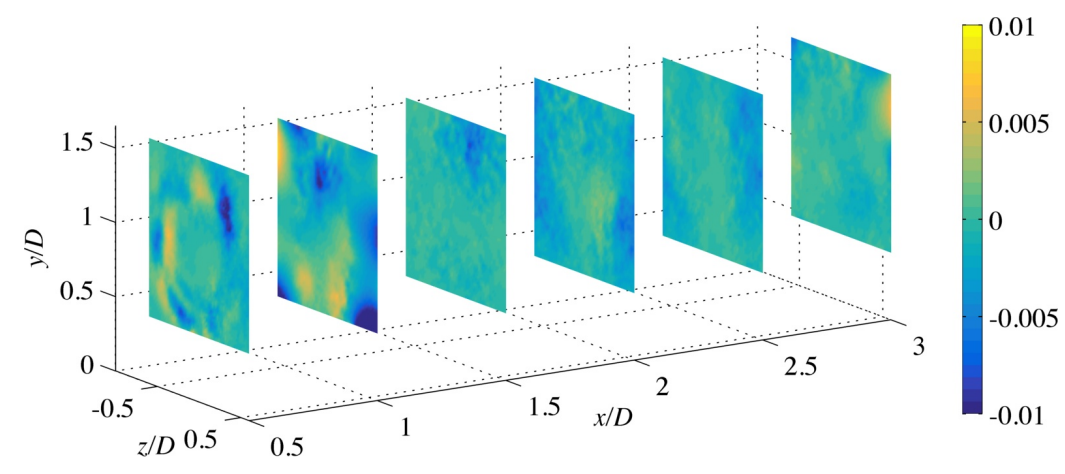

Figure 4.6: Contours representing the contribution of the total flux by the streamwise-wall-normal deterministic stress $-\overline{u^{\prime \prime} v^{\prime \prime}} U$.

in the wind turbine canopy. Phase-averaged contours of the streamwise velocity (not shown) are nearly identical to one another, regardless of phase of the rotor. The deterministic stresses, according to their standard definition, account for the deviation of phase-averaged velocities from the time-averaged velocities and are quite small.

However, the turbulent stress tensor demonstrates a large degree of variation with phase orientation of the rotor. Figures 4.65(A) and 4.65(B) show that the stress field varies considerably with phase. This variability is not accounted for in the definition of the deterministic stresses employed here. Assessment of the variability of the stress tensor is undertaken through the root-mean-square of the deviation from the phaseaveraged stresses from the time-averaged field according to equation (2.9), resulting in a tensor of tertiary stresses. The tertiary stresses are phase-independent and quantify the variation of the turbulence field arising from the passage of the rotor blades.

The formulation used is analogous to the definition of turbulence intensity, at least for the streamwise diagonal term $\overline{u^{\prime \prime \prime 2}}$ shown in figure 4.7(A). Deterministic turbulence intensity is shown to peak at $x / D=1$ following the same crescent shape as the turbulent stress. Very near to the model wind turbine, the contour plot of $\overline{u^{\prime \prime \prime 2}}$ shows the 


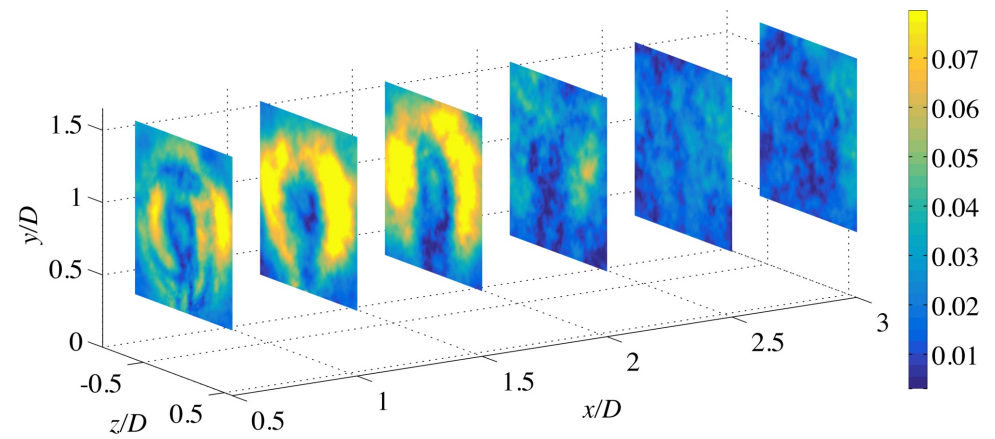

(A) $\overline{u^{\prime \prime \prime 2}}$

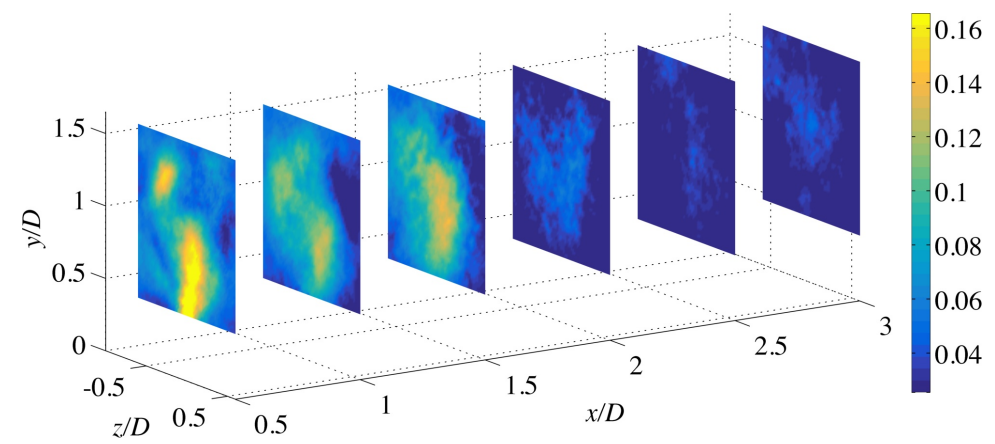

(B) $-\overline{u^{\prime \prime \prime} v^{\prime \prime \prime}}$

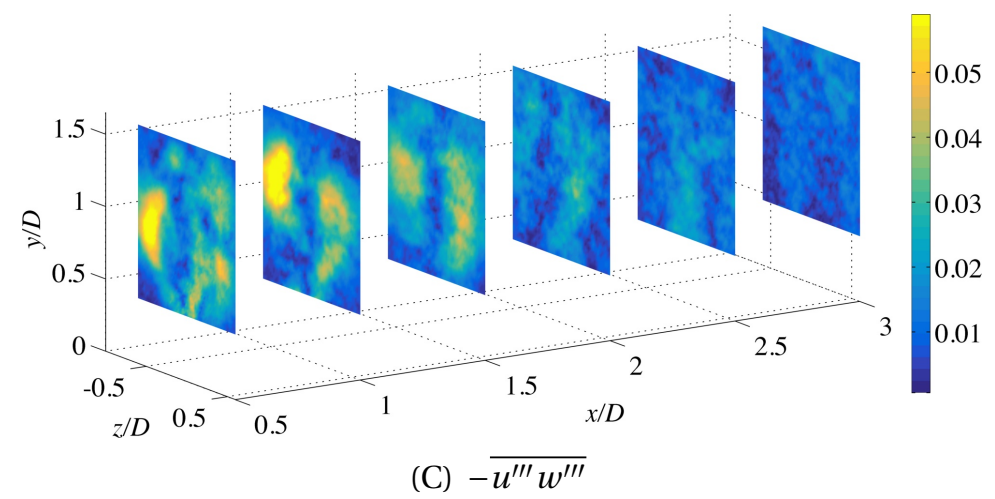

Figure 4.7: Tertiary stresses in the near wake. Shown in the figure are the streamwise contribution, analogous to deterministic turbulence intensity and the components that contribute to kinetic energy flux.

similar fine-scale features as the respective phase- and time-averaged fields.

Off-diagonal terms of the tertiary stress tensor must have a different physical interpretation; turbulence intensity is not defined for shear terms. Tertiary shear stresses 
are taken to express the energy added beyond what one would expect from of an equivalent aperiodic flow; contributions from off-diagonal components of $\overline{u_{i}^{\prime \prime \prime} u_{j}^{\prime \prime \prime}}$ account for asymmetry and periodicity that are missed in non-rotating geometry, such as porous discs often used in wind energy simulations. In the wake, shear terms of the tertiary stress tensor show equal orders of magnitude as the phase-averaged turbulence stresses above, which is indicative of their importance in the overall behavior of the wake. The tertiary stress composed of the streamwise-wall-normal components shows features that are quite distinct from the classically defined stresses. It is shown in the contours of $-\overline{u^{\prime \prime \prime} v^{\prime \prime \prime}}$ (figure $4.7(\mathrm{~B})$ ) that the deviation of phase-averaged fields from the Reynolds stress is negligible at $x / D \geq 2$. This is not the case for $-\overline{u^{\prime \prime \prime} w^{\prime \prime \prime}}$, shown in figure $4.7(\mathrm{C})$. Tertiary stress arising from the variation in the streamwise/spanwise turbulence stress conforms more closely to the regions of extreme $-\langle u w\rangle_{\alpha}$. The peak value of $-\overline{u^{\prime \prime \prime} w^{\prime \prime \prime}}$ occurs at $x / D=1$ corresponding to the peak negative value of $-\langle u w\rangle_{60^{\circ}}$. Tertiary stresses arise from phase-dependent variations in the turbulence field and make a significant contribution only near the turbine. Large scales of turbulence are expected to persist into the far wake but phase-dependence of the Reynolds stress tensor becomes difficult to diagnose beyond $x / D=1.5$.

While the global flux of mean flow kinetic energy into the turbine canopy by turbulence must be from above, local transport may occur laterally within the canopy. The results discussed here have been formulated in a Cartesian frame of reference, which aids the discussion of vertical transport into the wakes. The stress field contributing to the entrainment of kinetic energy is dominated by two components in this reference frame. A coordinate transformation to a polar-cylindrical frame with axis aligned with the hub of the model wind turbine effectively combines these components into 
a single term. Figure 4.8 demonstrates the transport processes discussed in the preceding results. Polar-cylindrical coordinates are more natural to the flow of a single wake, which is approximately axisymmetric but for the influence of the atmospheric boundary layer and the wake of wind turbine mast.

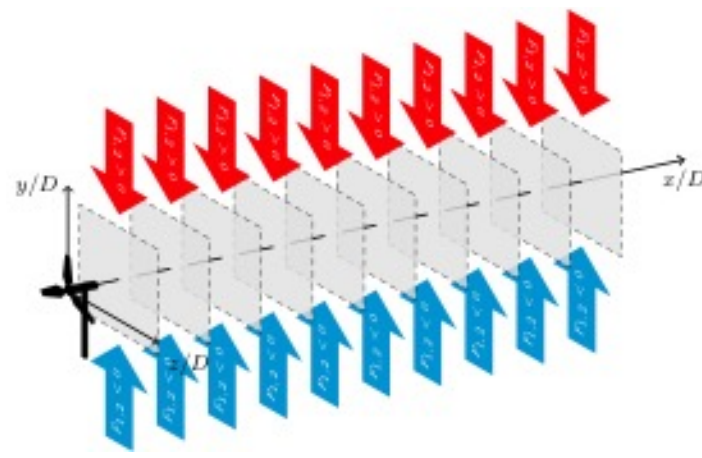

(A) Vertical entrainment of kinetic energy.

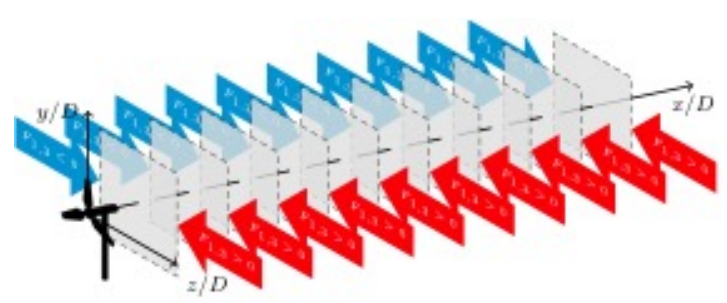

(B) Lateral entrainment of kinetic energy.

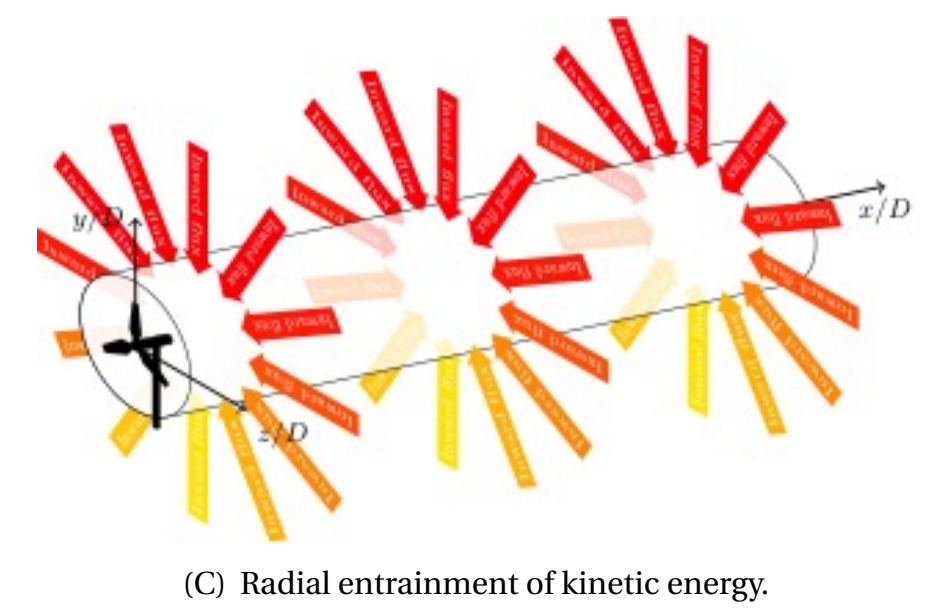

Figure 4.8: The entrainment process described in Cartesian and polar-cylindrical coordinate systems.

In the polar-cylindrical formulation, the component of the Reynolds stress tensor contributing to inward flux of kinetic energy is composed of fluctuations in velocity in the axial $\left(u_{x}\right)$ and radial $\left(u_{r}\right)$ directions. Fluctuating azimuthal velocity $u_{\theta}$ are contribute to redistribution of energy around the wake but does not contribute directly to 
wake remediation. The inward component of the flux then combines the mean axial velocity and the axial-radial turbulent stress and is denoted $F_{x r}=\overline{u_{x} u_{r}} U_{x}$. Figure 4.9 shows the inward flux, demonstrating that mean flow kinetic energy is transported toward the center of the wake everywhere except directly following the mast of the wind turbine. For $x / D \leq 2$, kinetic energy is moved outward, away from the center of the wake. The peak value of flux into the wake occurs trailing the top tip of the rotor at $x / D=1.5$ and is well described by the $-\overline{u v}$ component in the Cartesian frame.

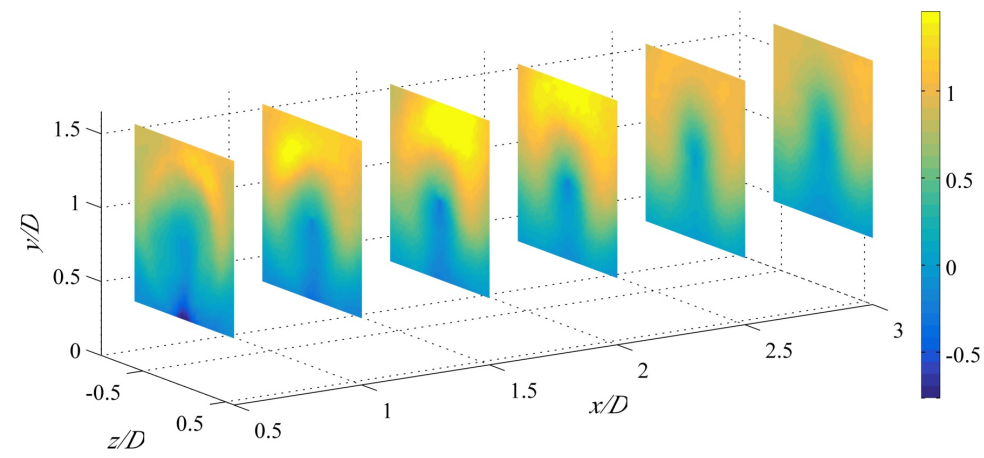

Figure 4.9: Contours representing the radial flux of mean kinetic energy into the wake of a wind turbine, $F_{x r}=\overline{u_{x} u_{r}} U_{x}$.

\subsubsection{Conclusions}

The data presented above result from a wind tunnel-scale experiment employing $2 D-3 C$ stereo PIV measurements in a fully-developed wind farm. Measurements were synchronized with the phase orientation of the rotor blades of a model wind turbine at four phase angles. Total mean values of axial velocity show the momentum deficit area in the near wake quite clearly but little dependence on the position of the rotor blades. The flux of kinetic energy is composed with the phase-averaged shear stress and compared showing that entrainment of high momentum flow is predominantly 
into the center of the wake. Following the rotor blades in certain orientations, there is flux of kinetic energy out of the wake below hub height, although evidence suggests that outward flux events are infrequent.

Deterministic stresses representing the combined deviations of phase-averaged velocities from the total mean quantities are approximately two orders of magnitude smaller than turbulent stresses. This difference in magnitude arises from the nature of the flow forcing the passage of the rotors, as opposed to other turbomachinery where the flow is forced by the blades. Although the deterministic contribution to the flux of kinetic energy is smaller in magnitude, there is some theoretical interest arising from azimuthal periodicity at the rotor diameter. That the deterministic stresses make a small contribution to the overall behavior suggests that they may merit further investigation.

An alternate decomposition directly quantifying deviations of phase-locked stresses from time-averaged values is of more interest in terms of the flux of kinetic energy. The third-order contribution to the entrainment of high momentum flow into the wake is accounted for in the tertiary stress tensor, where the streamwise normal component is formulated in a fashion analogous to the turbulence intensity, and quantifies variability of the turbulence field in the wake arising from the passage of the rotors. Shear terms of the tertiary stress tensor demonstrate quantitatively that periodic contributions to the turbulence field are highly variable. Tertiary stresses only make significant contribution near the turbine; the phase-dependence of the Reynolds stress tensor becomes difficult to diagnose beyond $x / D=1.5$. Tertiary stresses also account for contributions missed by fixed geometries, such as porous discs used in numerical simulations. 
The streamwise/spanwise tertiary stress $-\overline{u^{\prime \prime \prime} w^{\prime \prime \prime}}$ demonstrates peak values in locations highlighted by the classically defined turbulent stress. This is an indication that the phase-dependent variation of $-\overline{u w}$ yields periodic contributions to the lateral entrainment of kinetic energy into the wake. The streamwise/wall-normal tertiary stress shows peak values following the mast of the model wind turbine. While $-\overline{u v}$ contributes to the largest source of kinetic energy flux, the phase variation does not necessarily describe the periodic contributions seen for $-\overline{u w}$.

Although large-scale turbulent motions dominate in the near wake, it is the phasedependent variations of the mean velocities that contribute to the deterministic stresses. Because mean velocities show little variation according to phase, the deterministic stresses are small when compared to turbulence. Phase-related variations in the mean velocities are difficult to detect except very near the turbine. However, variations in large-scale turbulence are easily seen throughout the near wake and contribute to the tertiary stresses. Periodicity and rotation added to the mean flow decay moving away from the turbine faster than the phase variation imparted to the turbulence field itself, ultimately leading the deterministic stresses to exhibit smaller magnitudes than the tertiary stresses. In the far wake, variations in the Reynolds stresses related to phase of the rotor also decay and turbulence tends toward a largely homogeneous state. Residual phase dependence is difficult to detect in far-wake turbulence statistics indicating that large scales of turbulence that persist do not express dependence on the passage or orientation of the rotor.

Applying a coordinate transformation to the data presented above simplifies the discussion of kinetic energy flux for an individual wake. For a single wind turbine wake, results indicate that lateral flux cannot be neglected in wake remediation. Transforma- 
tion to polar-cylindrical coordinates yields a single component of the flux tensor that describes the entrainment of high-momentum flow into the center of the wake, radially inward from outside. The radial flux of kinetic energy $F_{x r}$ shows a crescent shaped feature similar to that of the streamwise normal stress. Antisymmetry shown by both $-\overline{u v}$ and $-\overline{u w}$ are combined in the transformation into the streamwise/radial shear stress $-\overline{u_{x} u_{r}}$.

Considering the flux of mean kinetic energy in polar-cylindrical coordinates demonstrates the tendency for inward transport, toward the core of the wake. Formulating the flux this way indicates that the wake itself may be represented as a sink term for energy. Analogous formulations for transport mechanisms of heat and moisture can account for the local drying of terrain seen in wind turbine arrays. Similar to the manner in which the kinetic energy flux entrains high-momentum flow from the outside, scalar fluxes (i.e. moisture and heat) are amplified by the turbulent flow in wind turbine wakes.

Tertiary stresses are a novel view of the variability of the turbulent wakes and would compliment other conditional statistics such as quadrant or octant analysis. Combined analysis would yield details regarding the frequency, intensity, and duration of phase-dependent turbulence events in wind turbine wakes. The streamwise-wallnormal stress is dominantly communicated through ejection events above the wind turbines canopy and through sweeps below the top tip of the rotors. These events are not clearly defined in the polar-cylindrical coordinate system demonstrated above. However, characterizing transport events radially inward and outward is of interest in detailing the entrainment of high-momentum flow toward the center of the wake. 


\subsection{Double proper orthogonal decomposition of a wind turbine wake}

\subsubsection{Wake flow characterization}

Flow statistics were calculated in each measurement plane, shown in figure 4.10. The measurement plane located at $x / D=3.5$ was corrupted by reflections that introduced noise to the mean statistics that intensified at higher order. It has therefore been excluded from the results below. Flow statistics are normalized by the incoming wind speed at hub height $U_{\text {hub }}=4.75 \mathrm{~m} / \mathrm{s}$, or by its square in the case of turbulent stresses. The mean streamwise velocity $U$ shows the characteristic momentum deficit of the wake in figure 4.63(A). As anticipated, the strongest momentum deficit is evident directly behind the nacelle of the wind turbine model at $x / D=0.5$. Recovery of the wake is seen progressing throughout the wake as $x / D$ increases. By $x / D=5$, the mean streamwise velocity increases monotonically with $y / D$, confirming the results from previous studies Hamilton et al. (2012). Gradients of $U$ across the wake are of equal order of magnitude in both the spanwise and wall-normal directions. As the wake recovers $(x / D \geq 4)$, the remaining wall-normal gradient is then obscured by the boundary layer that develops between rows of turbines in the array.

The largest single component of the Reynolds stress tensor is the streamwise normal stress $\overline{u u}$, shown in figure 4.10 (B). Very near to the device at $x / D=0.5$, the passage of the rotor is evident in the concentric circular arcs following the tip and root of the wind turbine blade. The mast is seen at $z / D=0$ in the near wake but is washed out beyond $x / D \approx 2.5$. The normal stress $\overline{u u}$ is most evident as a crescent-shaped struc-

The following results are taken from the recent publication, Hamilton, N., M. Tutukn, and R. B. Cal. "Low-order representations of the canonical wind turbine array boundary layer via double proper orthogonal decomposition.” Physics of Fluids (1994-present) 28.2 (2016): 025103. 


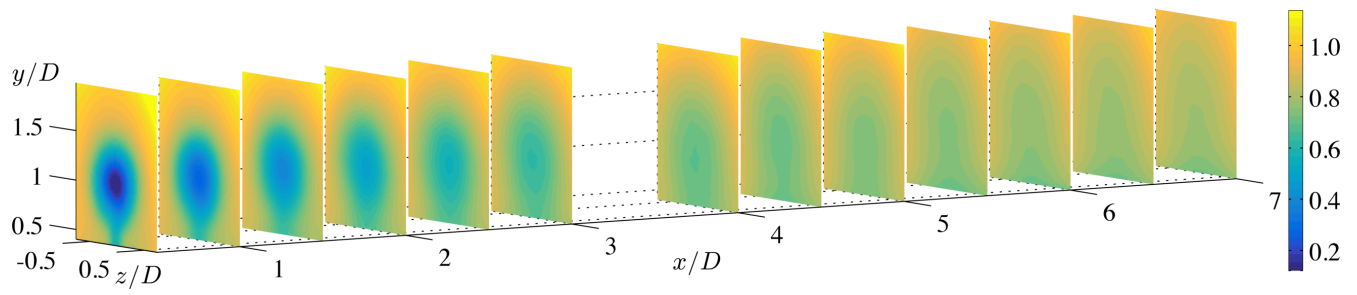

(A) Normalized mean streamwise velocity $U / U_{\text {hub }}$.

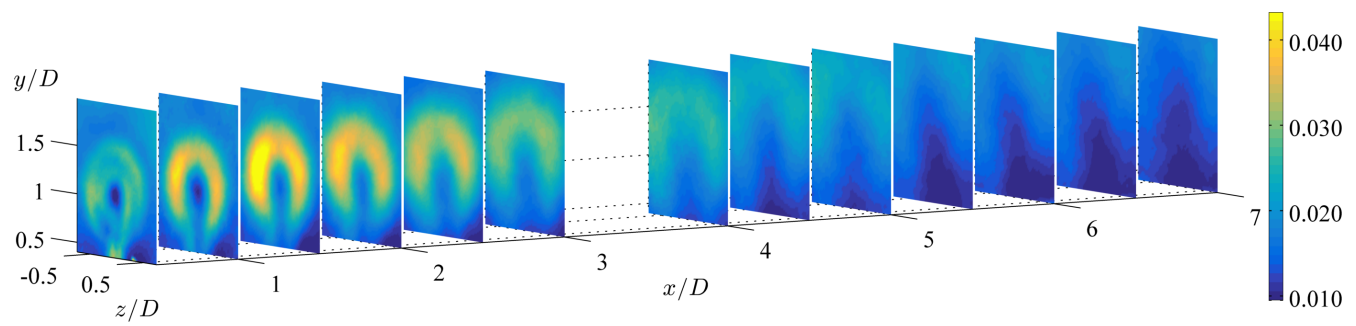

(B) Normalized streamwise normal stress $\overline{u u} / U_{\text {hub }}^{2}$.

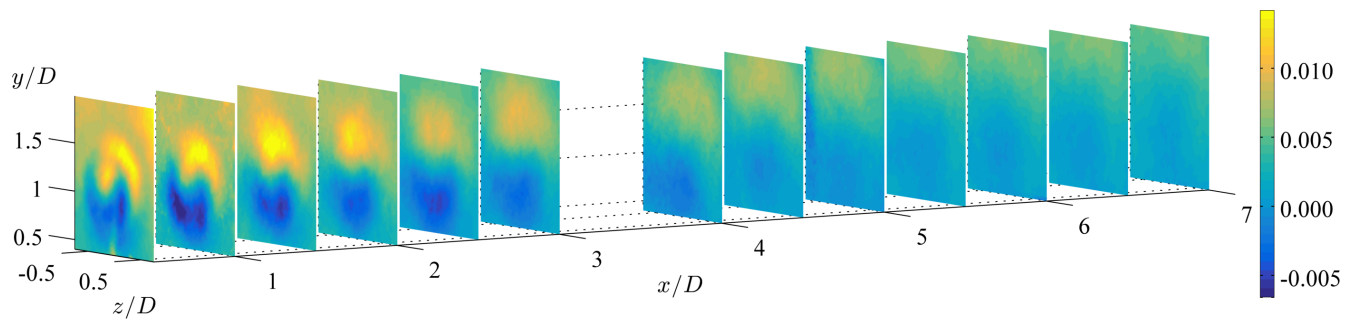

(C) Normalized shear stress $-\overline{u v} / U_{\text {hub }}^{2}$.

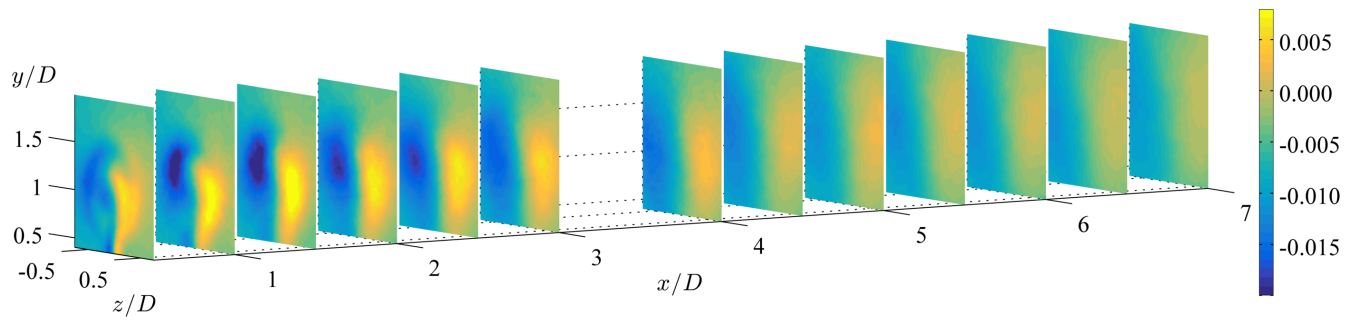

(D) Normalized shear stress $-\overline{u w} / U_{\text {hub }}^{2}$.

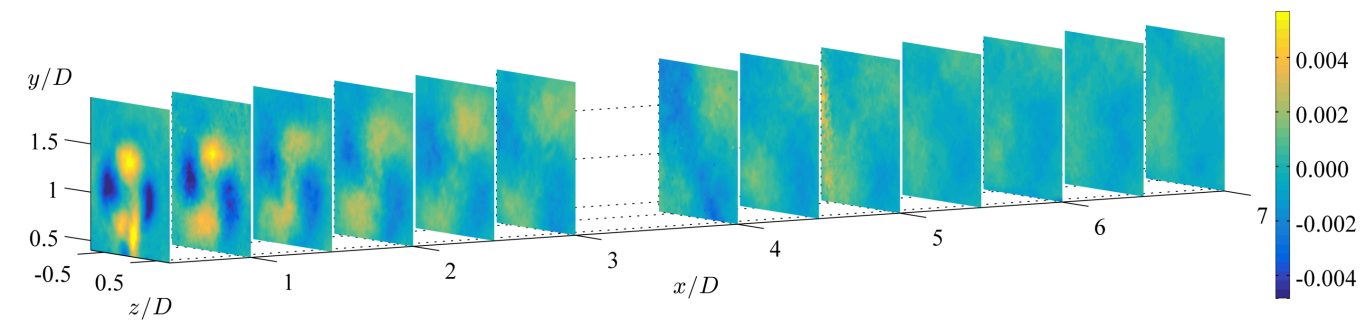

(E) Normalized shear stress $-\overline{v w} / U_{\text {hub }}^{2}$.

Figure 4.10: Flow statistics for wind turbine wake. Flow is from left to right. 
ture following the outer portion of the wind turbine blades above $y / D \approx 0.75$. In the far wake, $\overline{u u}$ is quite small and more homogeneously distributed throughout the measurement windows. The other diagonal components of the Reynolds stress tensor $\overline{v v}$ and $\overline{w w}$ (omitted from the current results for brevity) show spatial complexity only very near to the wind turbine.

Figures 4.65(A) through 4.65(C) show the turbulence shear stresses for the wake. Peak magnitudes of $-\overline{u v}$ and $-\overline{u w}$ are approximately $1 / 3$ the magnitude of $\overline{u u}$ but show similar spatial development throughout the wake. Figure 4.65(A) shows $-\overline{u v}$ to have a complicated structure reflecting the rotation of the rotor and the geometry of the blades in the near wake. There, the region of negative shear is confined to the area immediately following the rotor below hub-height. As the flow mixes, $-\overline{u v}$ becomes more evenly distributed, the negative shear stress becomes nearly even throughout the lower half of the wake. Magnitudes of the Reynolds shear stress in the positive region is three time more intense compared to that of the negative region. The difference of extremes confirms that the entrainment of high-momentum flow into the wake is predominantly downward from above. Reflecting the findings of previous studies Hamilton et al. (2015a,b), the region in which $-\overline{u v}>0$ drifts away from the wall in the far wake.

The streamwise-spanwise Reynolds shear stress is approximately antisymmetric about the $x y$-plane, shown in figure 4.65(B). As discussed above, $-\overline{u w}$ shows the effects of rotation of the rotor blade in the near wake as a sinuous line distinguishing the positive and negative contributions to the field. Like $\overline{u u}$ and $-\overline{u v}$, the peak value of $-\overline{u w}$ occurs at $x / D=1.5$. Along the streamwise coordinate the distinguishing line between positive and negative contributions is smoothed out although it maintains a 
slant toward the upper-left corner of each window. Current literature Cal et al. (2010); Calaf et al. (2010); Hamilton et al. (2012) discusses the entrainment process as occurring primarily in the vertical direction. This is true if considering the canopy as a single entity, as the vertical direction is the only unconstrained direction. However, if modeling of the near wake including rotational effects and lateral entrainment is desired, $-\overline{u w}$ cannot be neglected. Gradients of $U$ with respect to wall-normal and spanwise directions are the same order of magnitude in the near wake, thus the lateral flux of kinetic energy $F_{1,3}=-\overline{u w} U$ is nearly equal to the vertical flux $F_{1,2}=-\overline{u v} U$. In the far wake, the spanwise gradient of the mean velocity is nearly null while the wall-normal gradient remains due to presence of the wall, resulting in flux vertically downward. Due to the softening of gradients $\left(\frac{\partial U}{\partial z}\right.$ and $\left.\frac{\partial U}{\partial y}\right)$, production of turbulence kinetic energy is very small in the far wake.

The in-plane shear stress $-\overline{v w}$ is shown in figure $4.65(\mathrm{C})$. At its peak, $-\overline{v w}$ is an order of magnitude smaller than the streamwise normal stress. Very near to the wind turbine model, there is evidence of the mast extending the positive region of $-\overline{v w}$ to the bottom of the measurement window. The structures of $-\overline{v w}$ diffuse outward from the nacelle of the turbine with $x / D$. The in-plane component demonstrates the lowest magnitude of the Reynolds stress tensor and the smallest signal-to-noise ratio in the current measurements. Because the mean spanwise velocity falls off quite quickly, $w / W$ quickly becomes relatively large and shows the slowest statistical convergence. Measurement error can be seen at $x / D=4$, showing a slightly more intense negative region of $-\overline{v w}$ than the other planes, and $x / D=5$ where a reflection amplifies noise at $z / D \leq-0.5$. 


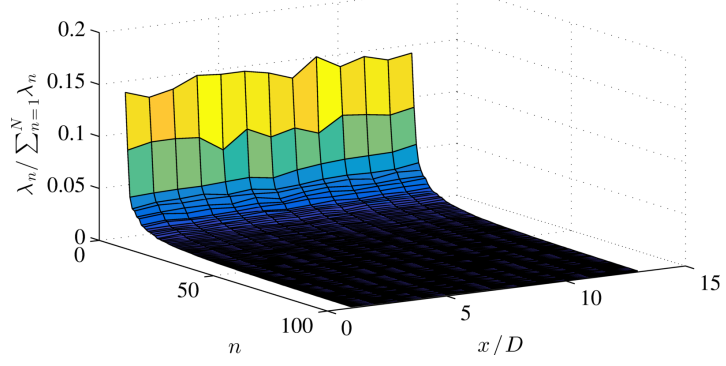

(A)

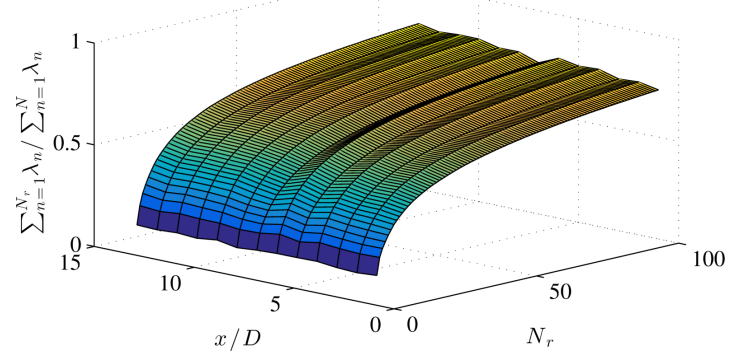

(B)

Figure 4.11: Eigenvalues of the POD $\left(\lambda^{(n)}\right)$ according to streamwise coordinate, figure 4.33(A). The normalized cumulative summation of eigenvalues is shown in figure 4.11(B). Only the first 100 eigenvalues are included.

\subsubsection{POD results}

The snapshot POD was applied to each measurement set separately, at each downstream location from $0.5 \leq x / D \leq 7$. Decomposition resulted in a total of 13 sets of POD modes $\Phi^{(n)}(x / D)$, each with 2000 modes. Additionally, eigenvalues $\lambda^{(n)}(x / D)$ for each set and the mode amplitudes used in velocity field reconstruction $a_{n}(x / D)$ are produced in the proper orthogonal decomposition. If each POD mode is considered a degree of freedom (DOF) of the turbulent flow in the wind turbine wake, the total basis of the measured system is formed of 26,000 DOF, 13 locations $\times 2000$ modes each.

The complexity of the turbulent wake makes modeling the full range of dynamics and behavior prohibitively difficult, if not impossible. The eigenvalues associated with each mode delineate the relative importance and integrated turbulence kinetic energy expressed by velocity field projections onto each mode. Figure 4.33(A) shows the distribution of eigenvalues as a surface in $x / D$ and mode number $n$. The cumulative fraction of $k$ is shown in figure 4.11(B) for the first 100 modes at each measurement location. 


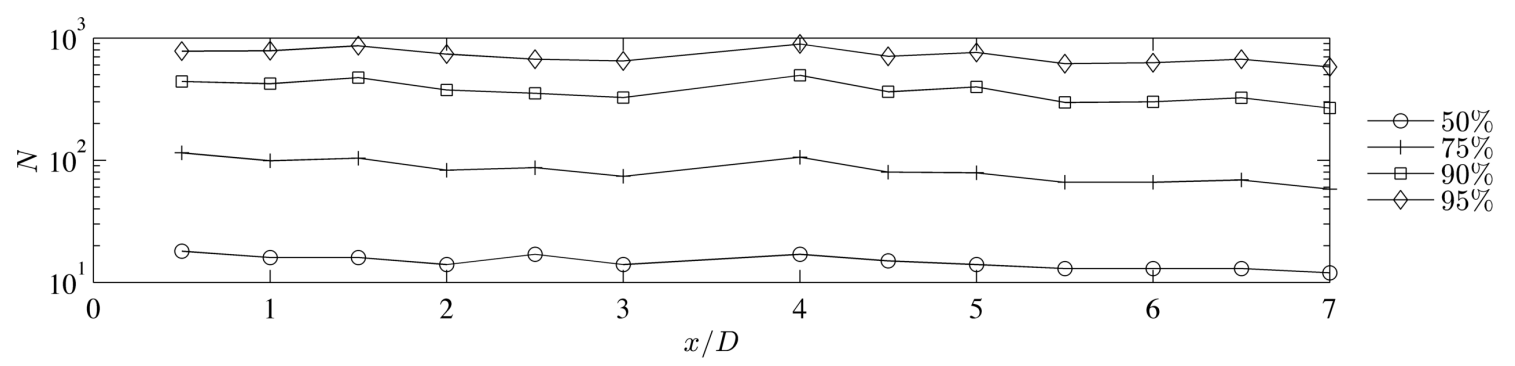

Figure 4.12: Cumulative fraction of $\lambda$ according to streamwise coordinate. The number of modes required to reach the listed thresholds are nearly constant in the wake.

Common practice with the POD is to use the sequence of eigenvalues to determine at which point a truncation to the basis may be made, reducing the complexity of the flow description. It is quite common to set an arbitrary threshold of energy as the truncation point. Figure 4.12 shows the number of modes required to account for given thresholds of TKE throughout the wake. Note that very few modes $(\approx 13)$ are required to reach the $50 \%$ of the energy in the measurement set, whereas many are required to restore $90 \%$ of the energy $(\approx 350)$.

Figures 4.13 shows the first POD mode associated with streamwise, wall-normal and spanwise directions for each measurement location. In the near wake, evidence of the rotor is seen in every component of the modes and rotation can be seen throughout the wake as slight asymmetry about $z / D=0$ into the far wake. Beyond $x / D \geq 2$, it is difficult to associate any particular structure in the modes to the passage of the rotor blades. The streamwise and wall-normal components of the first POD modes are roughly antisymmetric in the far wake. There the spanwise component $\phi_{w}^{(1)}$ is nearly homogeneous in the measurement area with the exception of the shear layer present at the uppermost edge of the wake at $y / D \approx 1.75$. Antisymmetry seen in the stress $-\overline{u w}$, plotted in figure 4.65(B), is visible in the streamwise component of the first POD mode 

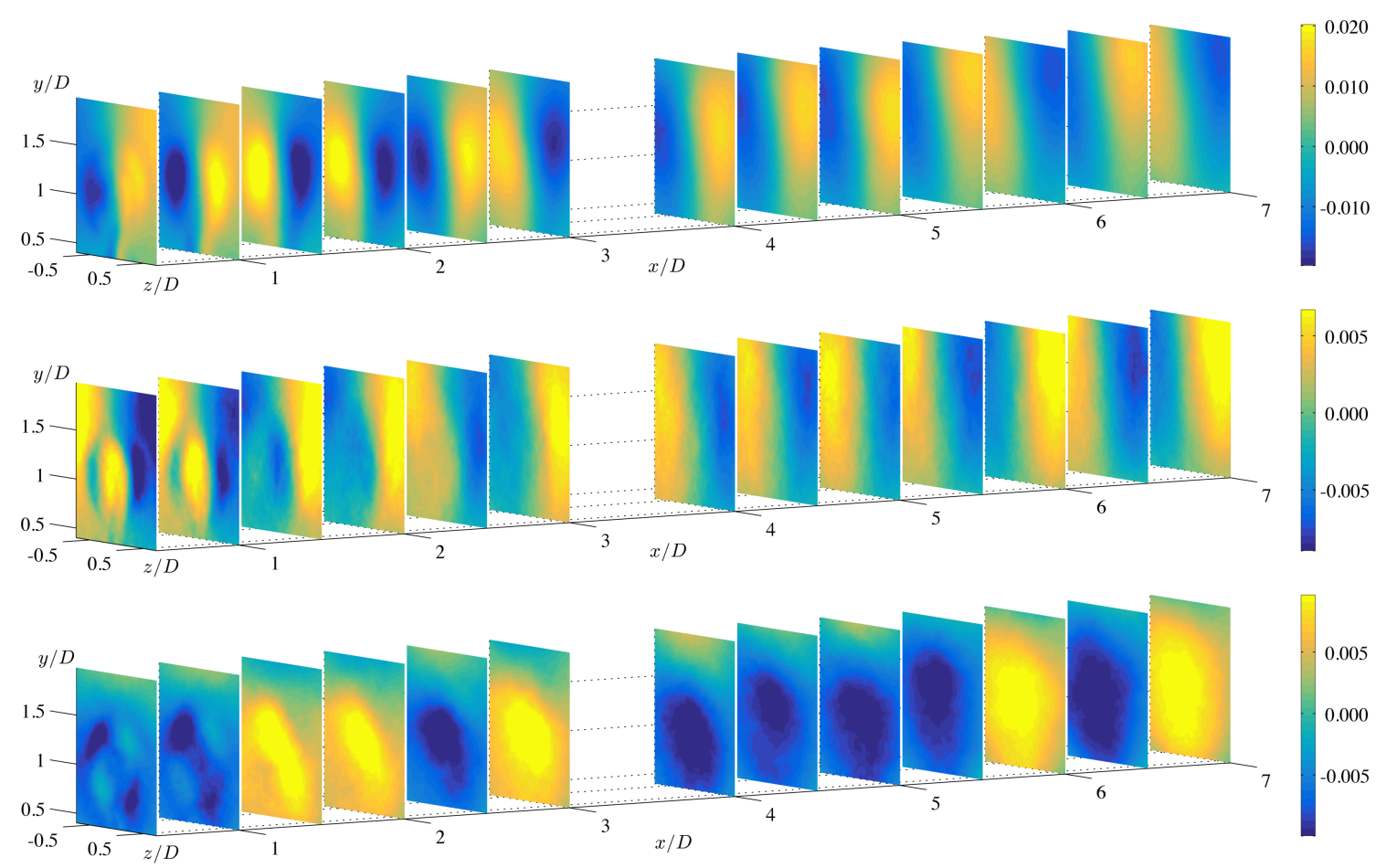

Figure 4.13: POD mode 1 in the wake. From top are the $\phi_{u}, \phi_{v}$, and $\phi_{w}$ components.

$\phi_{u}^{(1)}$. The quadrant-like structure seen in $-\overline{v w}$ is seen in $\phi_{w}^{(1)}$.

Low modes are remarkably consistent throughout the wake along $x / D$. The streamwise component of the first POD mode $\phi_{u}^{(1)}$ is roughly antisymmetric about the center of the field at $z / D=0$. However, the algebraic sign of the mode changes along $x / D$. The sign dependence of the modes is arbitrary in the basis but is corrected in reconstruction with the respective amplitudes $a_{n}(x)$. The amplitudes can take either positive or negative values and in linear combination with the modes lead to a algebraically consistent contribution to the Reynolds stress tensor.

The second POD mode seen in figure 4.14 is structurally independent of the first mode as the formulation of the POD requires that each mode maintains orthogonality with the others. The vertical component $\phi_{v}^{(1)}$ is expressed in a single peak with con- 
sistent magnitude and is centered in the spanwise direction following the wind turbine. As with $\Phi^{(1)}$, the second mode does demonstrate variation in the very near wake related to the passage of the rotors. The streamwise component $\phi_{u}^{(1)}$ exhibits a large structure above hub-height that contributes to the structure of $-\overline{u v}$. The relationships of the modes to the Reynolds stress tensor are otherwise not as clear as for $\boldsymbol{\Phi}^{(1)}$.

The flexibility of the algebraic sign shown in the first mode is also seen in $\boldsymbol{\Phi}^{(2)}$. Interestingly, the locations that favor one sign over another are not consistent from mode to mode (e.g. at $x / D=1.5)$. Although generally considered to have the least significance and associated energy in the wake, the spanwise component $\phi_{w}^{(2)}$ shows more complexity than the other components. At this mode number, there are still clear common projections in each snapshot basis. According to the cumulative summation of eigenvalues seen in figure 4.11(B), $\boldsymbol{\Phi}^{(1)}$ and $\boldsymbol{\Phi}^{(2)}$ together account for approximately $25 \%$ of the integrated turbulence kinetic energy.

The intermediate range of POD modes is characterized by a loss of common projections. For modes above $n \approx 15$ it becomes very difficult to distinguish any coherent features. Figure 4.15 shows $\boldsymbol{\Phi}^{(100)}$, chosen as a representative mode of intermediate mode number. The eigenvalues provide a measure of the importance of each mode in the total flow. Normalized eigenvalues are consistent for all measurement locations and rapidly decrease in order of magnitude. The eigenvalues associated with the first mode $\left(\lambda^{(1)}(x / D) \approx 0.14\right)$ and the hundredth mode $\left(\lambda^{(100)}(x / D) \approx 0.0003\right)$ indicate the relative importance of each mode. As for $\Phi^{(100)}$, the modes of intermediate $n$ do not exhibit structural consistency in the wake.

Truncating the POD basis and reconstructing the stresses according to equation (4.19) yields Reynolds stresses filtered to include only the energetic turbulence struc- 

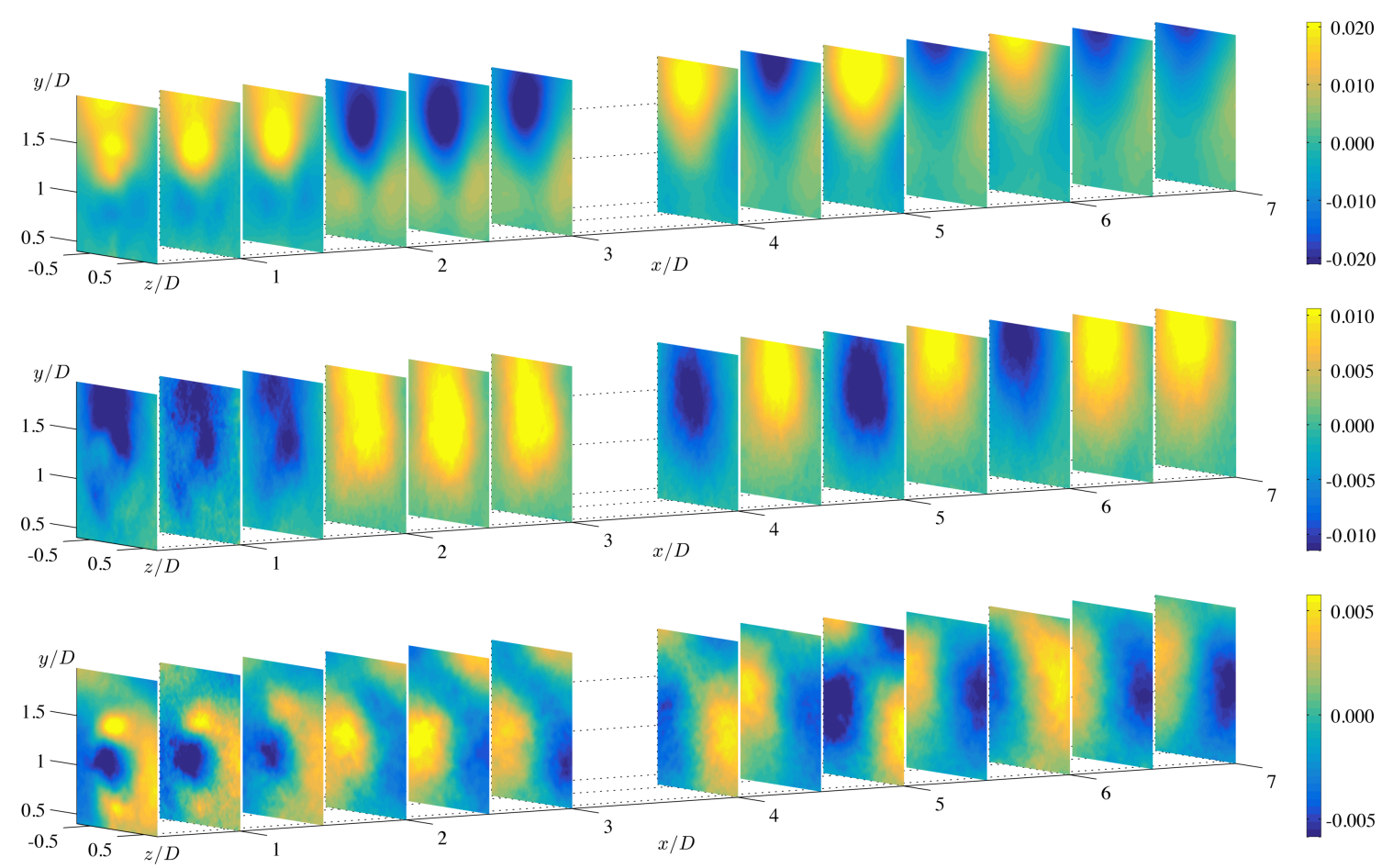

Figure 4.14: POD mode 2 in the wake. From top are the $\phi_{u}, \phi_{v}$, and $\phi_{w}$ components.

tures. Figure 4.16 shows reconstructions of three stresses using a local threshold of $50 \%$ TKE (circle markers in figure 4.12). The reconstruction of $\left.\frac{\circ}{u u}\right|_{50 \%}$ in figure $4.16(\mathrm{~A})$ exhibits the crescent-shaped structure more clearly than the original statistics, since some of the small scale structures have been omitted. At $x / D=0.5$, the reconstruction shows two lobes rather than the concentric rings in the original contour plot. In the far wake $x / D \geq 4$, the crescent is more angular than the unfiltered statistics, arising from the truncated basis used in the reconstruction. A maximum of 14 modes are required to reach the $50 \%$ thresholds used here according to the trend in figure 4.12 .

Shear stresses reconstruct much more ably than the normal stress discussed above. Figures 4.16(B) and 4.16(C) are quite similar in structure and magnitude to the original unfiltered statistics. The figure also shows that complex asymmetric structures arising 


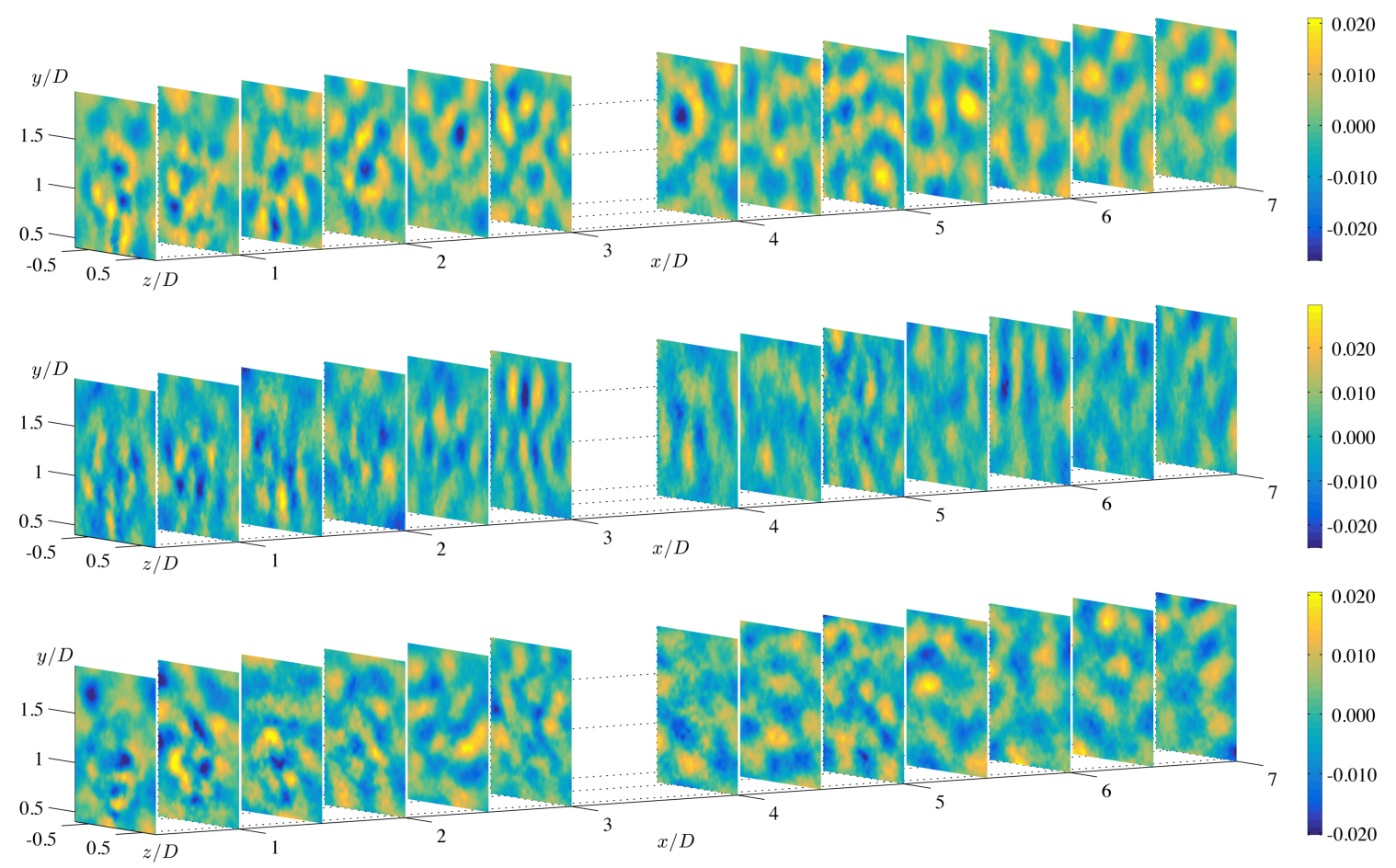

Figure 4.15: The 100th POD mode, $\boldsymbol{\Phi}^{(100)}$. Spatial organization into large structures is not evident in any but low mode numbers. From top are the $\phi_{u}, \phi_{v}$, and $\phi_{w}$ components.

from the passage of the rotors are represented well with a reduced POD basis. The shear stresses $\left.\frac{0}{u v}\right|_{50 \%}$ and $\left.\frac{0}{u w}\right|_{50 \%}$ are nearly identical to the conventional turbulence statistics at $x / D=0.5$. This is also the location of the most structurally complex flow in the wake.

\subsubsection{DPOD results}

Modes shown above in figures 4.13 through 4.15 were concatenated into bases of fixed mode number to form the bases for the DPOD. The procedure follows the work by Siegel et al. (2008) with the exception that DPOD now offers decomposition over a spatial coordinate, further reducing the dependence of the resulting sub-modal basis to 


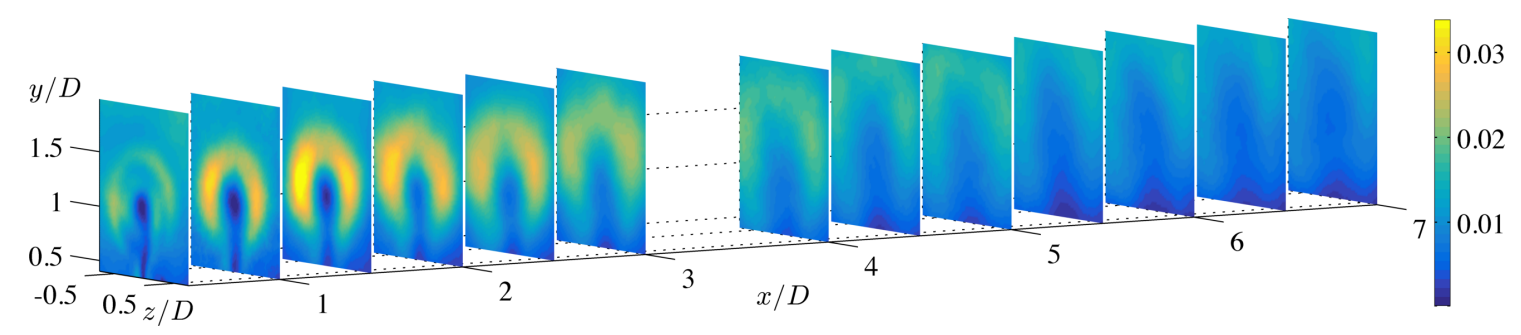

(A) $\left.\stackrel{\circ}{\mathrm{u} u}\right|_{50 \%} / U_{\mathrm{hub}}$.

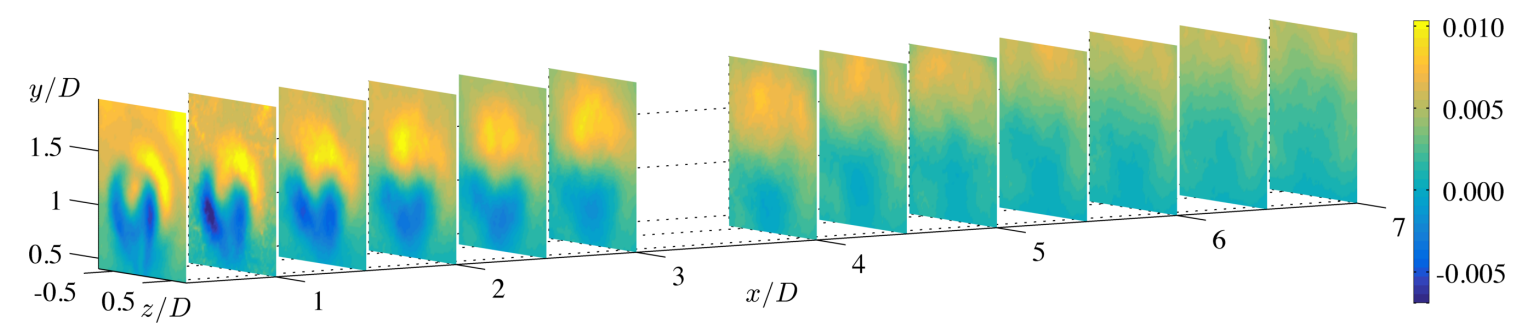

(B) $\left.\stackrel{\circ}{u v}\right|_{50 \%} / U_{\text {hub }}$.

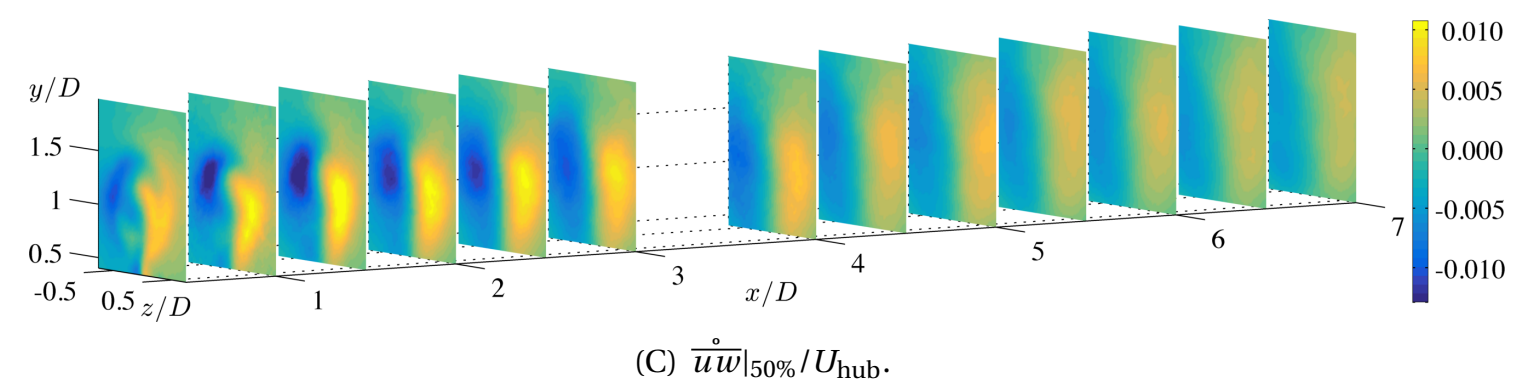

Figure 4.16: Reconstructions of turbulence stresses based on 50\% energy delineated by the cumulative fraction of $\lambda$.

the in-plane spatial coordinates. The streamwise dependence is then accounted for by the series of amplitudes $b_{n, m}(x)$. Time dependence of the basis is already accounted for by the amplitudes of the original snapshot POD $a_{n}(t)$. Application of the POD over concatenated sets of modes of common rank is shown as the step from point (C) to (D) in the conceptual diagram found in figure 2.2.

Secondary application of the POD was the chosen method of assessing the evolution of modes in a streamwise sense due to its nature to filter important structures to 
front of the vector basis and less important structures to the end. Additionally, as the POD works through eigenvalue decomposition and linear combination, it effectively ignores the switching of the algebraic signs seen above. Figures 4.13 and 4.14 demonstrate the organization and structural similarity of modes along the wind turbine wake. That the intermediate and high mode numbers do not show the same degree of organization throughout the wake is not surprising; less coherent turbulence structures that lack the energy necessary to persist throughout the wake.

Unlike the original application of the POD, the mean value of the kernel was not removed before decomposition. Therefore, the first mode in the resulting set of basis functions is the largest common projection between the POD modes and is representative of the mean of each mode,

$$
\boldsymbol{\Gamma}^{(n, 1)} \approx\left\langle\boldsymbol{\Phi}^{(n)}\right\rangle
$$

The plots of $\boldsymbol{\Phi}^{(1)}$ and $\boldsymbol{\Phi}^{(2)}$ (figures 4.13 and 4.14) indicate that although the structure of the POD modes shows a consistent form throughout the wake, the algebraic signs of the modes often switches. During the reconstruction of fluctuating velocity fields, the mode amplitudes $a_{n}$ can take on negative values which accounts for the differences in mode signs from one measurement location to the next. However, the sign switching renders conventional statistics inadequate, as averaging a sign switching field may lead to erroneous results.

As with the POD, the DPOD yields a set of orthogonal basis functions $\left(\boldsymbol{\Gamma}^{(n, m)}\right)$ and associated eigenvalues $\Lambda^{(n, m)}$ that communicate the relative importance of each basis function. It should be stated that in the first POD applied directly on the velocity 


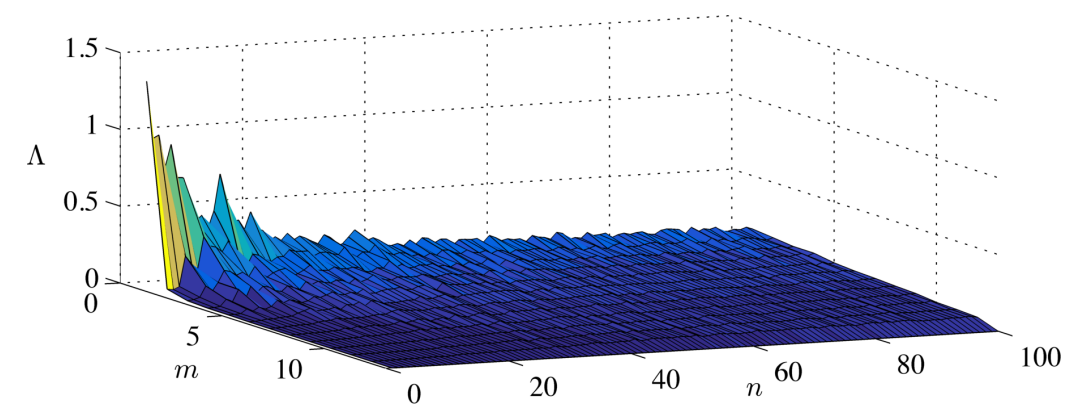

Figure 4.17: Distribution of eigenvalues associated with each sub-modal structure in wind turbine wake.

field, the eigenvalues $\lambda^{(n)}$ relay the relative importance of $\Phi^{(n)}$ in terms of energy. Because the snapshot basis of the DPOD is made of unitless eigenmodes of the first decomposition, the eigenvalues resulting from the DPOD $\Lambda^{(n, m)}$ are unitless as well. The span of DPOD eigenvalues $\Lambda^{(n, m)}$ is shown in figure 4.17. The figures illustrates that the weighted distribution expressed by the DPOD eigenvalues is a function of both the POD mode number $n$ and sub-mode number $m$. For low mode numbers ( $n$ and $m$ ), the modes are organized in the wake and share structural elements. Organized modes have a few important substructures and $\Lambda^{(n, m)}$ falls off quickly. Increasing mode number yields structures that are less organized throughout the wake. At higher mode numbers, the distribution of $\Lambda^{(n, m)}$ is nearly homogeneous across the span of substructures. The flattening of the distribution implies that the sub-modal projections become more equal and that there are fewer identifiable trends in the POD mode in the wake.

To illustrate the significance of the sub-modes outlined in figure 4.17 , several DPOD bases are shown in the following figures. The decomposition of $\boldsymbol{\Phi}^{(1)}$ into sub-modal structures shown in figure 4.18 illustrates the consistency of the modes in the wake. The distribution of $\Lambda^{(1, m)}$ shows that the importance of the sub-modal structures pro- 
gressing in $m$ varies over orders of magnitude. The sub-modes are well organized for low sub-modal numbers but lose coherence and appear noisy for $m \geq 6$. Figure 4.18 shows the full span of $\boldsymbol{\Gamma}^{(1, m)}$. Each plane is now a sub-modal component forming the basis of $\boldsymbol{\Phi}^{(2)}$. The three components of the vectorial sub-modes are shown in the same format as the POD modes above for consistency, from top are the spans of $\gamma_{u}^{(1, m)}, \gamma_{v}^{(1, m)}$, and $\gamma_{w}^{(1, m)}$. The ordinate now represents the sub-mode number $m$ rather than the streamwise coordinate. The first plane in each subfigure is the effective mean mode throughout the wake, immune to the switching of algebraic sign seen in figure 4.13. Following the effective mean structure are corrections to the modal bases in order of significance of projection. The second and third sub-modes correspond well with features seen in the near wake of $\boldsymbol{\Phi}^{(1)}$. Areas roughly sketching the swept area of the rotor are highlighted in sub-modes $m=2,3$, and 5 in $\gamma_{u}$ and $\gamma_{v}$ but are not as clear in $\gamma_{w}$. That the first sub-mode does not show effects from the rotor indicates that the nearwake features are secondary to the underlying wake structure.

Similar levels of organization are seen in the span of $\Gamma^{(2, m)}$. The first sub-modes for $m \leq 4$ demonstrate coherent and organized features within the measurement fields but higher sub-mode numbers, the coherence is lost and the fields appear noisier. As with $\Gamma^{(1, m)}$, the sub-modes of $\boldsymbol{\Phi}^{(2)}$ can be thought of as a 'starting' structure (effective mean, first sub-mode, $m=1$ ) followed by progressively detailed adjustments. Several of the sub-modes exhibit Fourier-type behavior seen especially in the third sub-mode $\Gamma^{(2,3)}$. Applying the decomposition over homogeneous data results in a simplification of POD modes to Fourier modes Citriniti and George (2000); Lumley (1967); Tutkun et al. (2008). The second POD mode displays some homogeneity, especially related to the vertical component $\phi_{v}^{(2)}$, and some Fourier-like behavior in the sub-modal struc- 

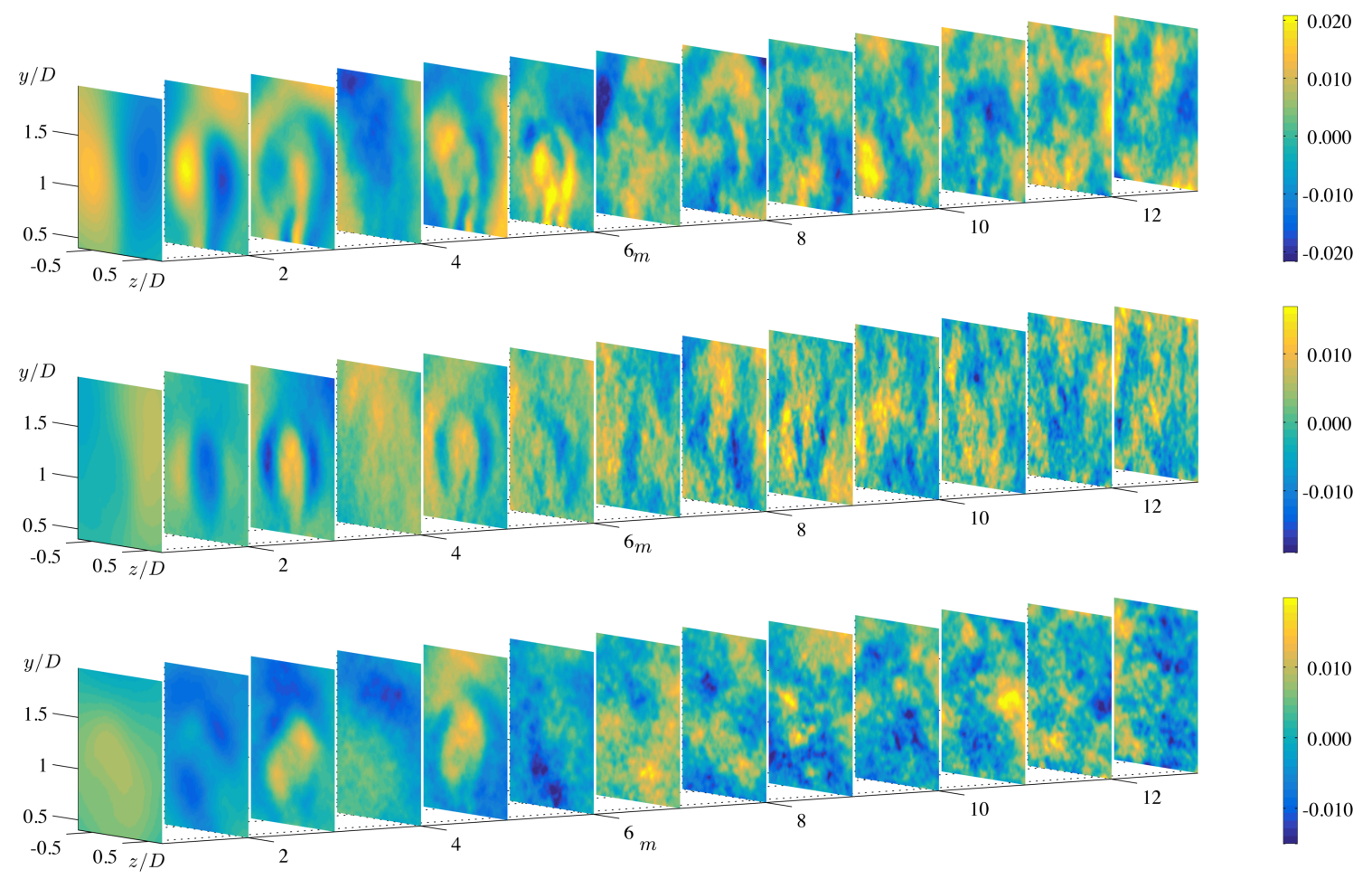

Figure 4.18: Span of the first set of DPOD modes, $\Gamma^{(1, m)}$. From top are the $\gamma_{u}, \gamma_{v}$, and $\gamma_{w}$ components.

tures is expected. However, this type of behavior is not seen in all sub-modes.

Sub-modes of intermediate POD mode numbers such as $\Gamma^{(100, m)}$ demonstrate no discernible change in organization across the span of $m$, validating the hypothesis that only the first few POD modes carry energy through the wake. Comparing the POD mode from figure 4.15 to the DPOD modes in figure 4.20 , one sees distinct differences from mode to mode, but not more organization. This indicates that there are no common projections of the POD modes characterized by intermediate and higher values of $n$ and that not much new information is gained by application of DPOD.

Similarity of projections is also evident in the flattening of distributions of $\Lambda^{(n, m)}$ in figure 4.17 . When this is the case, the reconstruction amplitudes associated with sub- 

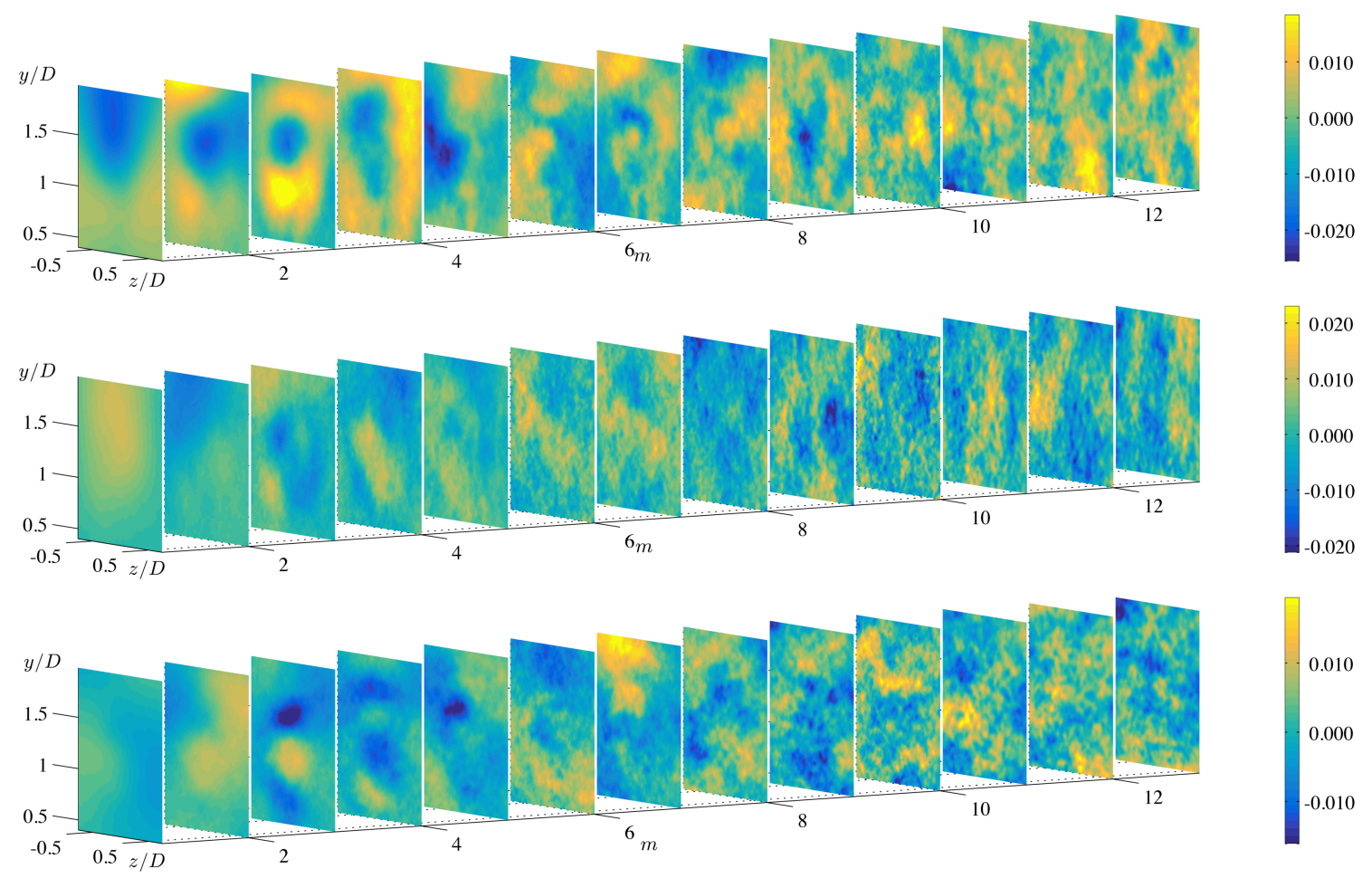

Figure 4.19: Span of the second set of DPOD modes, $\Gamma^{(2, m)}$. From top are the $\gamma_{u}$, $\gamma_{v}$, and $\gamma_{w}$ components.

modal structures $b_{n, m}$ do not show any preference for low sub-mode numbers and are more uniform across the span of $m$. The span of sub-mode amplitudes is shown in figure 4.21 where the amplitude $b_{n, m}$ decays with $m$ for low mode number $n$. At high mode number, $b_{n, m}$ shows no obvious dependence on $m$.

The reconstruction amplitudes are important in reconstructing the POD modes from a truncated set of sub-modal structures according to equation (2.26). Each POD mode may be represented as a linear combination of sub-modes with amplitudes like those shown in figure 4.21. The amplitudes in the figure do not convey units as they do with the POD amplitudes $a_{n}$. An important point regarding $b_{n, m}$ is that the amplitudes are functions of the streamwise coordinate while the DPOD modes themselves 

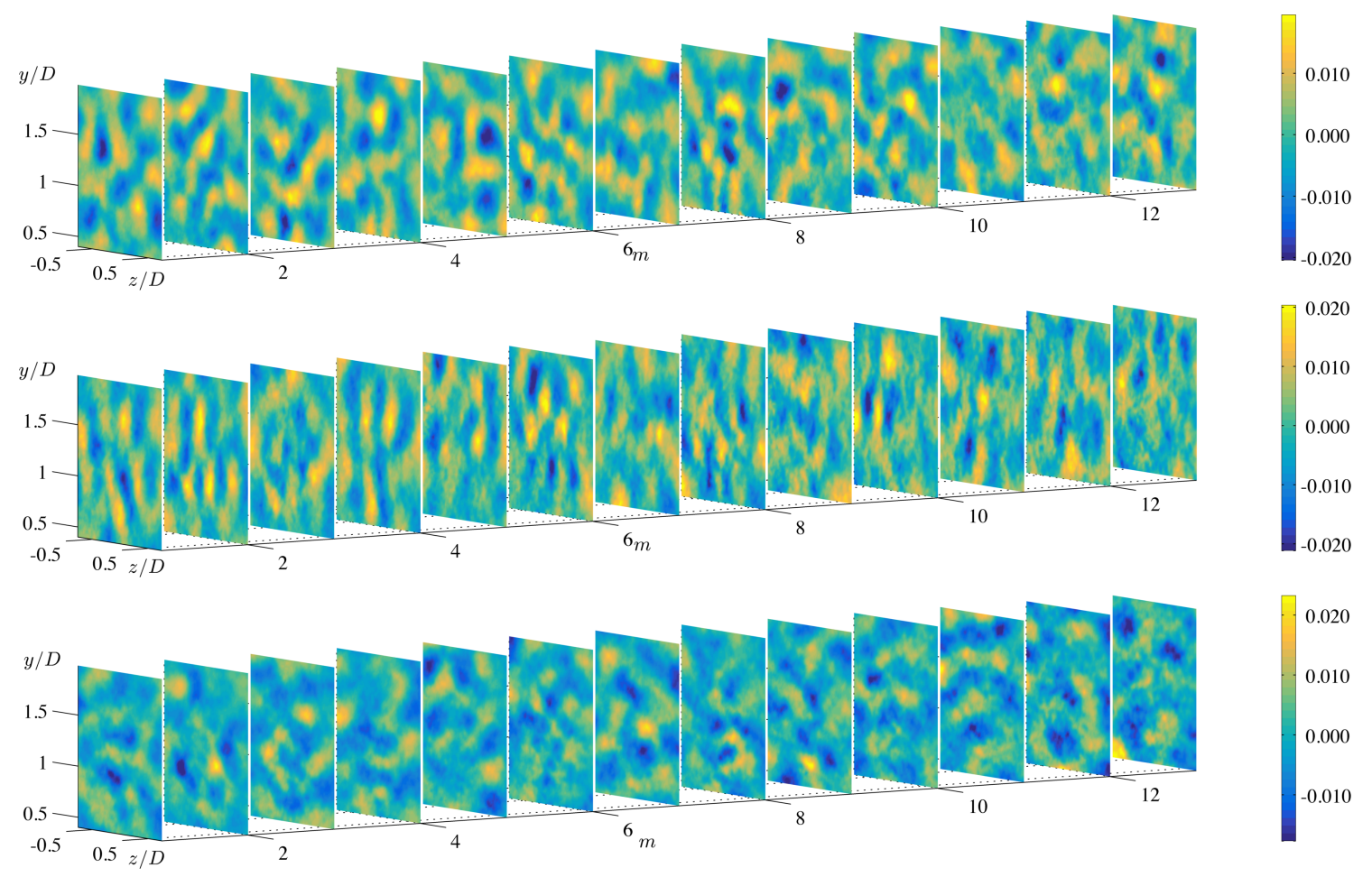

Figure 4.20: DPOD of intermediate POD mode, $\Gamma^{(100, m)}$. From top are the $\gamma_{u}, \gamma_{\nu}$, and $\gamma_{w}$ components.

are not. This implies that evolution of the original POD modes in the wake may also be described by the amplitudes $b_{n, m}$.

The span of sub-modes $\Gamma^{(n, m)}$ can be truncated as for the POD. In doing so, the less organized sub-modal structures are omitted and linear combination then yields filtered POD modes $\stackrel{\circ}{ }^{(n)}$. The sub-modes that are omitted are at least two orders of magnitude less significant than $\Gamma^{(1,1)}$. Because a series of filtered POD modes is the desired outcome of the DPOD, each mode must be reconstructed with a truncated set of DPOD modes rather than using the method that reconstructs the kernel directly (equations (2.25) and (2.26)). In this case, the correlation tensor of the POD modes $D^{n}$ is not the desired output but a series of low-order descriptions of the POD modes 


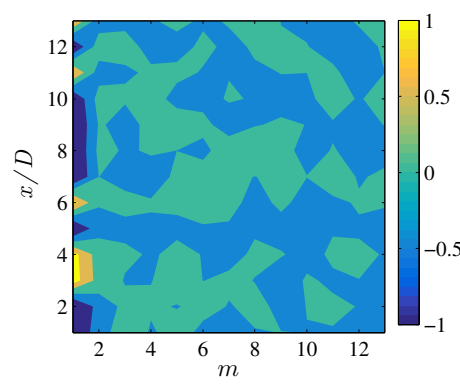

(A)

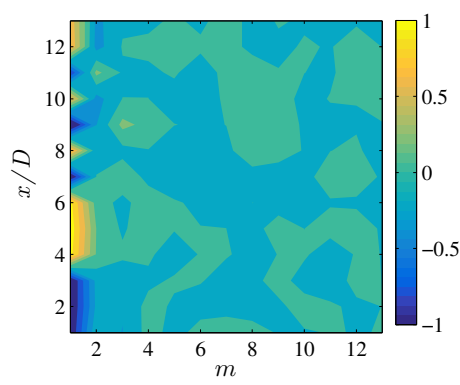

(B)

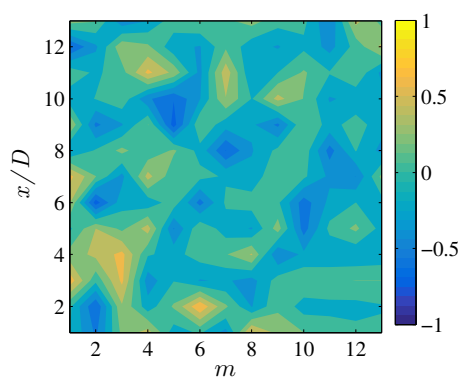

(C)

Figure 4.21: Sub-modal amplitudes $b_{n, m}$ associated with (a) $\boldsymbol{\Gamma}^{(1, m)}$, (b) $\boldsymbol{\Gamma}^{(2, m)}$, and (c) $\Gamma^{(100, m)}$.

themselves. The subspace of the reconstruction amplitudes is shown in figure 4.21. Mentioned earlier, the amplitudes $b_{n, m}$ account for any sign switching seen in the POD modes.

Reconstructions of Reynolds stresses with POD modes at different degrees of filtering illustrate the contribution made by the first several sub-modes. Figure 4.22 compares the original statistical shear stress $-\overline{u v}$ (left column) firstly to the low-order description with unfiltered POD modes. Filtering at the POD level $-\stackrel{\circ}{u v}$ (second from left) is able to accurately rebuild the Reynolds stress tensor with only a few modes. However, despite similarity of either the original stress $-\overline{u v}$ or the stresses filtered at the POD level $-\frac{\circ}{u v}$, each downstream location must be reconstructed individually with independent sets of modes and eigenvalues. The double POD outlined above further reduces the necessary modes from $N_{r} \times 13$ degrees of freedom to a set of $N_{r} \times M_{r}$ submodes without considerable loss in accuracy.

Representations employing more sub-modes (relaxing sub-modal filters) in the description of statistics approach $-\left.\frac{\circ}{u v}\right|_{n}$ with increasing span of $m$. The near-wake measurement locations show that the stress decreases in magnitude and that the effects of 
rotation decrease rather quickly. This is the range of the wake that exhibits the most complexity in the turbulence field. Looking to the third, fourth, and fifth columns of figure 4.22, it is evident that the corrected stress reconstructed with sub-modal filter-

ing $\left(-\left.\frac{\circ}{u v}\right|_{2} ^{m}\right)$ is nearly identical from one location to the next. Increasing the number of sub-modes used to describe the basis functions leads to more distinct reconstructed stresses.

Structures of $-\left.\frac{\circ}{u v}\right|_{2} ^{m}$ appear to change with $m$, but quantitative comparison of $-\overline{u v}$ and the filtered descriptions in the following section indicates that they are in fact quite similar. Evidence suggests that the accuracy of reconstructions does not change significantly beyond a few modes either at the POD or the DPOD levels. This is taken as indication that the flow is dominated by only a few coherent turbulent structures that are well-characterized by the DPOD. The following section details a direct method of correcting the range of dynamics in low-order descriptions of the Reynolds stress tensor with drastically reduced bases. The observation that the main features of the turbulence stresses in the wake may be reconstructed with very few modes suggests that the energy excluded must be fairly uniform or isotropic.

\subsubsection{Corrected low-order description}

In the context of the current work, the primary point of concern is finding a simplified means of describing the turbulent dynamics in wind turbine wakes. Increasing the number of POD modes used in the description increases the percentage of the turbulence kinetic energy represented. Successively more complex models (by the number of modes used) approach the original statistics used in the kernel.

POD applied to wind turbine wakes, discussed in Hamilton et al. (2015b), was 


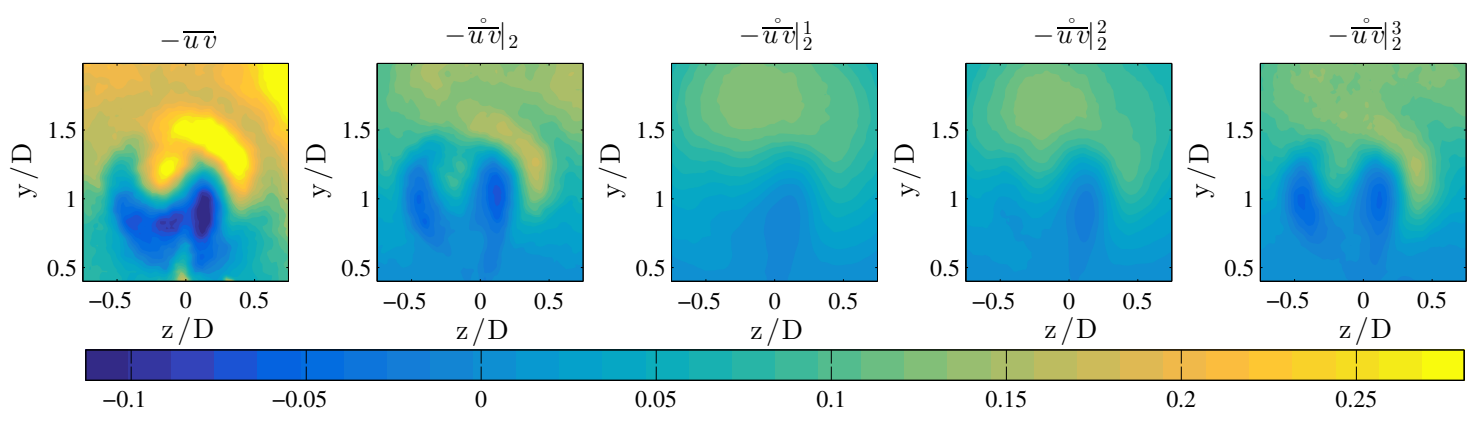

(A) Reconstruction with sub-modal filtering at $x / D=0.5$

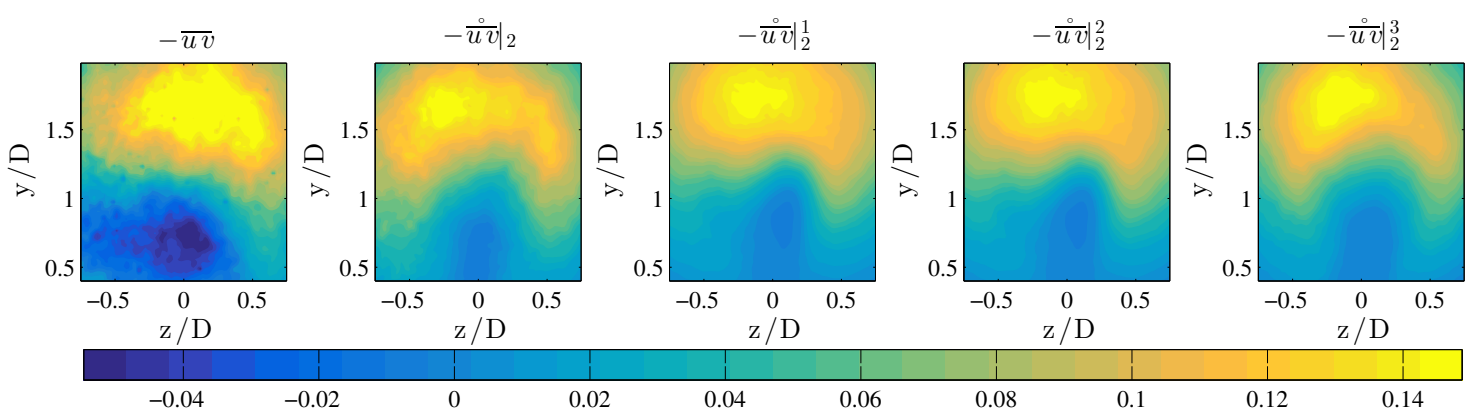

(B) Reconstruction with sub-modal filtering at $x / D=3.5$

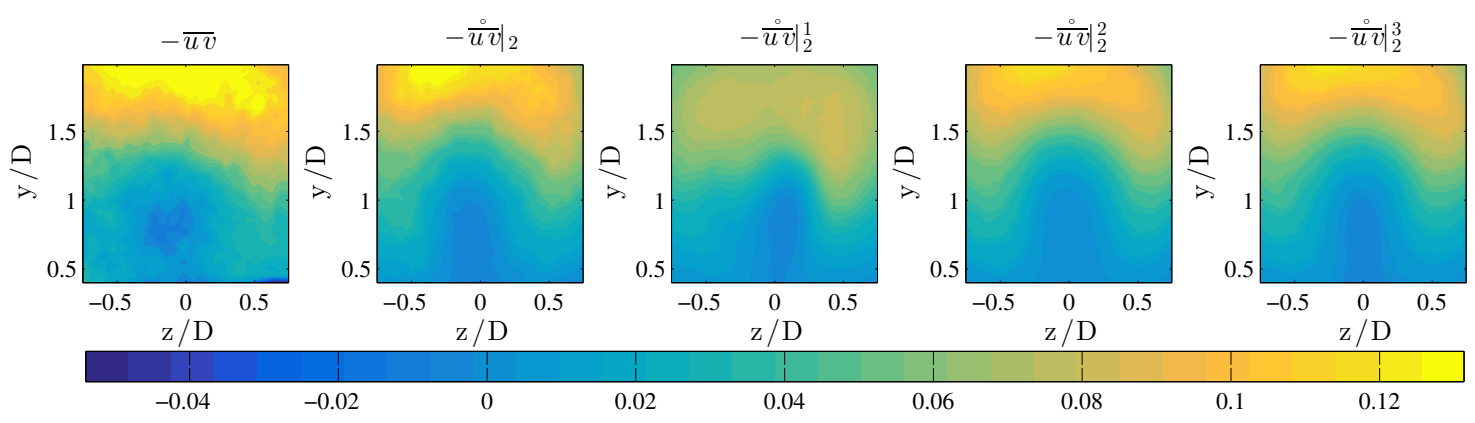

(C) Reconstruction with sub-modal filtering at $x / D=6$

Figure 4.22: Comparisons of the shear stress $-\overline{u v}$ based on varying levels of submodal filtering. From left are the original statistical stress, the POD reduced order description, and the stress filtered with 1, 2, and 3 of the 13 sub-modes. 
shown to make a fair approximation of original statistics with as few as $1 \%$ of the total modes. In that work, the streamwise average of $-\overline{u v}$ and the associated components of the flux $(-\overline{u v} U)$ and production $\left(-\overline{u \nu} \frac{\partial U}{\partial y}\right)$ were analyzed in order to characterize the wake and approximations of it. A similar approach is undertaken below with specific profiles of the Reynolds stresses. Spatial averaging was determined to be inappropriate in the context of the current measurement set and aim of the model. Here, measurements are across the wakes and the in-plane spatial gradients are important considerations in the overall behavior of the wake.

Profiles of $\overline{u u}$ and $-\overline{u v}$ are shown in figure 4.23 are compared to profiles of the same stress modeled with bases reduced to varying amounts. In the figure, profiles in black represent quantities derived statistically and come directly out of figure 4.10. Gray profiles (light and dark) are statistics composed with a truncated basis of POD modes. As the number of modes increases, the POD representations approach the profile of the original statistics as expected. Using a single POD mode (profiles shown in dark gray solid lines) to represent the stress field in the wake is unable to capture the shape or character of the original statistics. The complex turbulence field in the wake cannot be reduced to a single eigenfunction. However, a description of the wake using two POD modes (profiles shown in dark gray dashed lines) is sufficient to reproduce the shape of the wake, if not the magnitudes of each stress. Profiles in light gray solid lines are those of composed with between 3 and 10 POD modes and demonstrate the tendency toward unfiltered statistics with larger modal bases.

This observation confirms that the lowest POD modes accounting for the large turbulence structures are responsible for the features of turbulence in the wake. Further, it confirms the hypothesis that reducing the modal basis is equivalent to excluding en- 

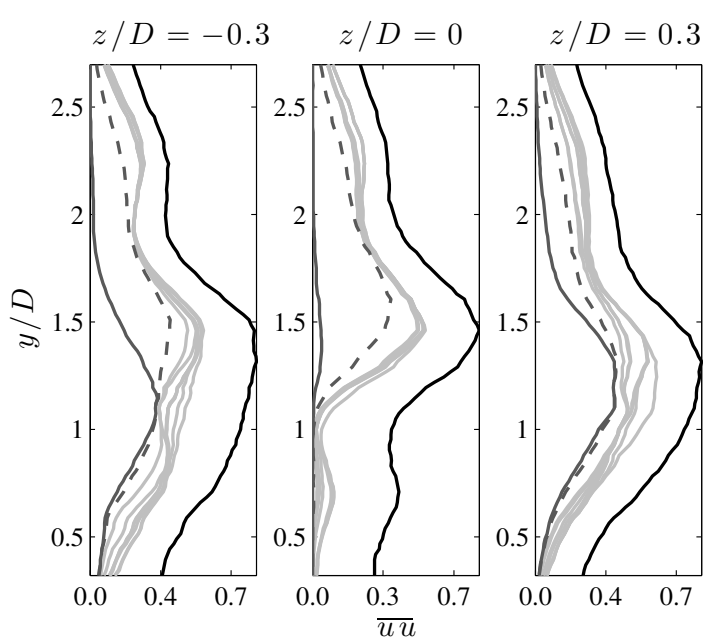

(A) Vertical profiles comparing $\stackrel{\circ}{u u}$ to $\overline{u u}$.

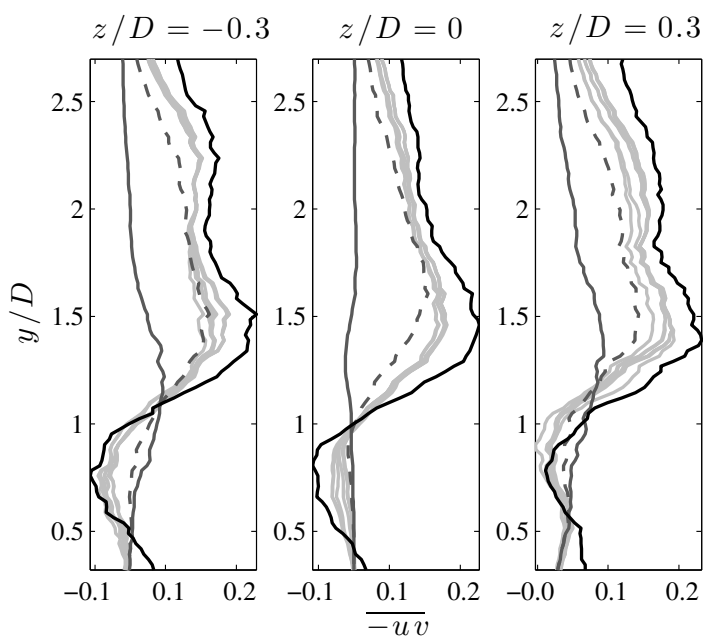

(C) Vertical profiles comparing $-\frac{\circ}{u v}$ to $-\overline{u v}$.
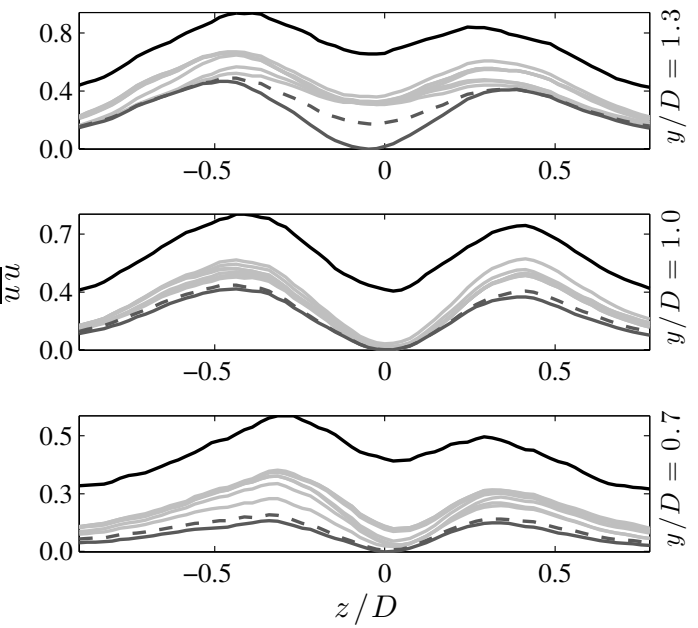

(B) Horizontal profiles comparing $\stackrel{\circ}{u u}$ to $\overline{u u}$.
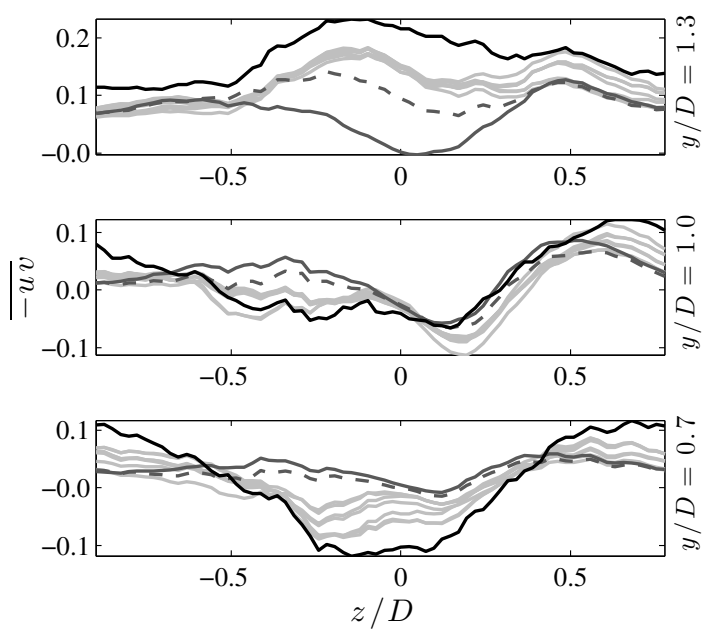

(D) Vertical profiles comparing $-\frac{\circ}{u v}$ to $-\overline{u v}$.

Figure 4.23: Convergence of $\frac{\circ}{u u}$ and $-\frac{\circ}{u v}$ to original statistical profiles using progressively more modes in reconstruction. Low-order descriptions with a single POD mode are shown as solid dark gray lines; descriptions with two POD modes dark gray dashed lines. Higher order descriptions are shown in light gray lines and the original statistics are shown in black. Profiles correspond to the data at $x / D=1.5$, where the highest magnitudes of Reynolds stresses occur.

ergy homogeneously from the wake. This suggests that a correction may be made to the low-order descriptions in the form of a simple constant to account for the turbu- 
lence kinetic energy in the excluded eigenvalues. The correction factor is a tensor of the form,

$$
\overline{u_{i} u_{j}}=\left.C_{i j} \bar{\circ} \overline{u_{i} u_{j}}\right|_{n}
$$

In the equation (4.22), the correction coefficient $C_{i j}$ is found through a minimization of the root-mean-square (RMS) error $\operatorname{err}_{i j}$ between the statistical stress field and the corrected low-order description.

$$
C_{i j} \ni \min \left[\sqrt{\left\langle{\overline{u_{i} u_{j}}}^{2}-\left(\left.C_{i j} * \frac{\circ}{u_{i} u_{j}}\right|_{n}\right)^{2}\right\rangle}\right]=\min \left[e r r_{i j}\right]
$$

The coefficient used to correct the range of statistics is a second-order tensor corresponding to the Reynolds stress tensor. The tensor $C_{i j}$ accounts for the energy in the POD modes and $\lambda$ values that are not used in the low-order description. Therefore, the expected behavior of the coefficient tensor is toward unity increasing the truncated basis used in the low-order description. The coefficient space for the corrected loworder description is shown in figure 4.24 and confirms that $C_{i j}$ approaches unity and that the RMS error tends toward zero as more POD modes are used in the low-order description; otherwise expressed as,

$$
\begin{aligned}
\lim _{n \rightarrow N} C_{i j} & =1 ; \\
\lim _{n \rightarrow N} e r r_{i j} & =0 .
\end{aligned}
$$

Figure 4.24 compares the necessary correction and associated error for compo- 


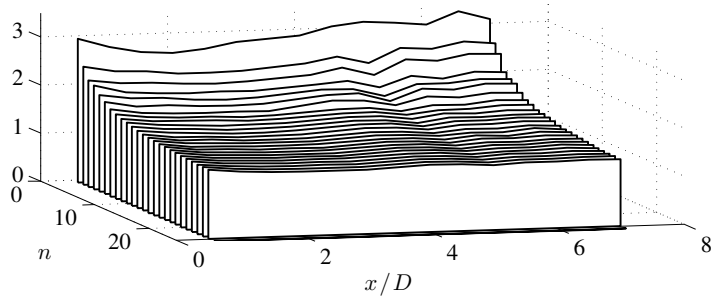

(A) $C_{11}$

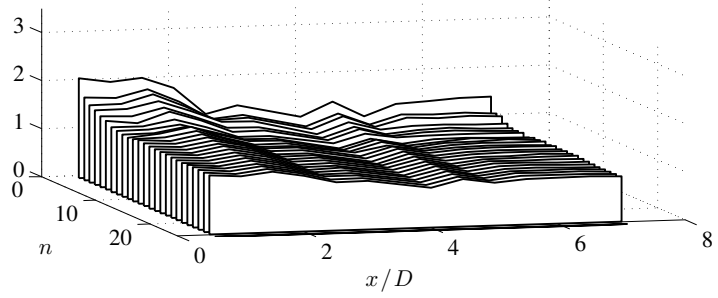

(C) $C_{12}$

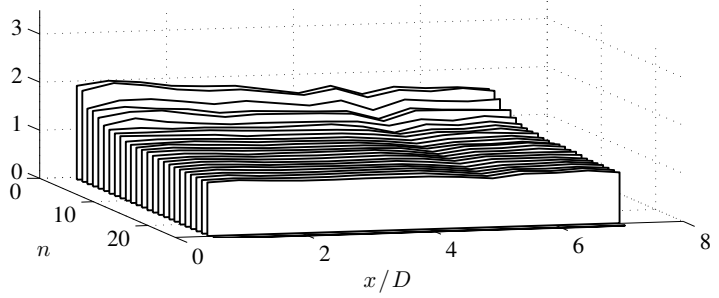

(E) $C_{13}$

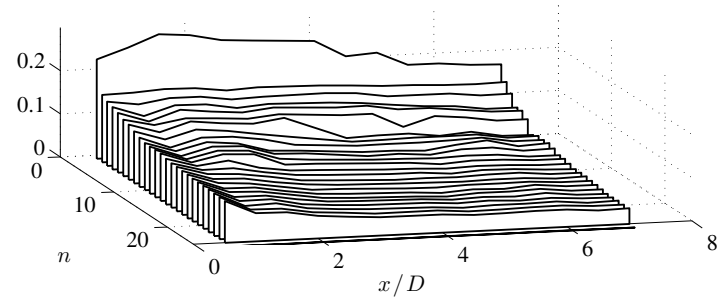

(B) $e r r_{11}$

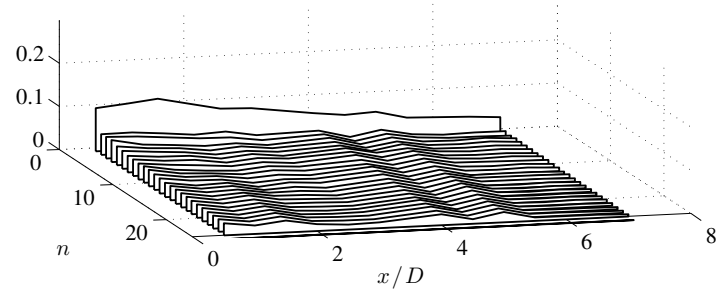

(D) $\operatorname{err} 12$

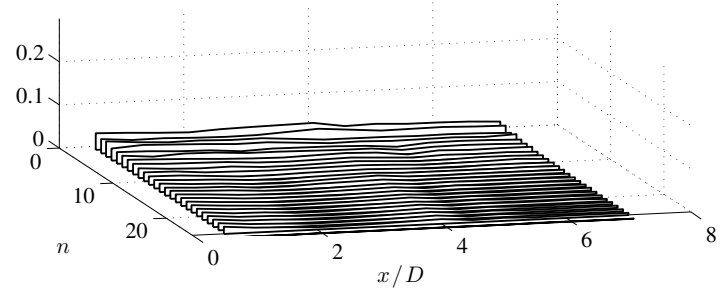

(F) $\operatorname{err}_{13}$

Figure 4.24: Coefficients (left column) and error (right column) associated with correction of low-order descriptions of the streamwise normal stress $\left(\frac{\circ}{u u}\right)$ and shear stresses responsible for entrainment $\left(-\frac{\circ}{u v}\right.$ and $\left.-\frac{\circ}{u w}\right)$ as a function of number of modes used in low-order representation of stresses $(n)$ and streamwise coordinate $(x / D)$.

nents of the Reynolds stress tensor involving fluctuations in the streamwise velocity. The coefficient space indicates that both the correction and the RMS error are less for shear terms than for normal stresses. Including successively more POD modes aids in the reconstruction of the Reynolds stress tensor unevenly; it takes many modes to rebuild the diagonal components of $\overline{u_{i} u_{j}}$ (normal stresses), whereas the character of 
shear terms is evident quite quickly, and the coefficient is near unity. The balance of the coefficients is necessarily dependent on the nature of the flow and may be further explored through an analysis of the turbulence anisotropy akin to that presented in Hamilton and Cal (2015).

Development of $C_{i j}$ and $e r r_{i j}$ associated with limiting the coefficient space to two POD modes is shown in figure 4.25. The basis is limited according to the above observation that a minimum of two modes are necessary to reproduce the form of the stress field in the wake. Figure 4.25(A) shows that the correction needed for many of the stresses is nearly constant throughout the wake. This is taken as confirmation that the POD modes in the wake are related to one another and their evolution may be described by additional simplifications. The exceptions to this observation are coefficients associated the vertical and spanwise normal stresses. In these cases, $C_{22}$ and $C_{33}$ are more variable in the wake in the range of $4 \leq C_{i j} \leq 6$.

While $C_{i j}$ is nearly constant for each stress, the error associated with the correction (figure 4.25(B)) decreases slightly as the wake develops in the streamwise direction. Also clear in the figure is the distinction between accuracy of the low-order description of shear and normal stresses. In the near wake, the error in describing the diagonal terms of $\overline{u_{i} u_{j}}$ is greater than $15 \%$, difficult to consider a good approximation for any model. Recalling that only $0.1 \%$ (two modes) of the POD basis is being used, the RMS error is surprisingly low. More impressive is that the reconstruction of shear terms is accurate to less than $5 \%$ even at $x / D=0.5$ where the stress field is structurally complex. The error of the low-order descriptions decreases in a roughly linear manner to approximately $3 \%$ for the shear stresses and in the range of $9-14 \%$ for the normal stresses. Seen in figure 4.23(A), the low-order representations of the normal stress $\overline{u u}$ 


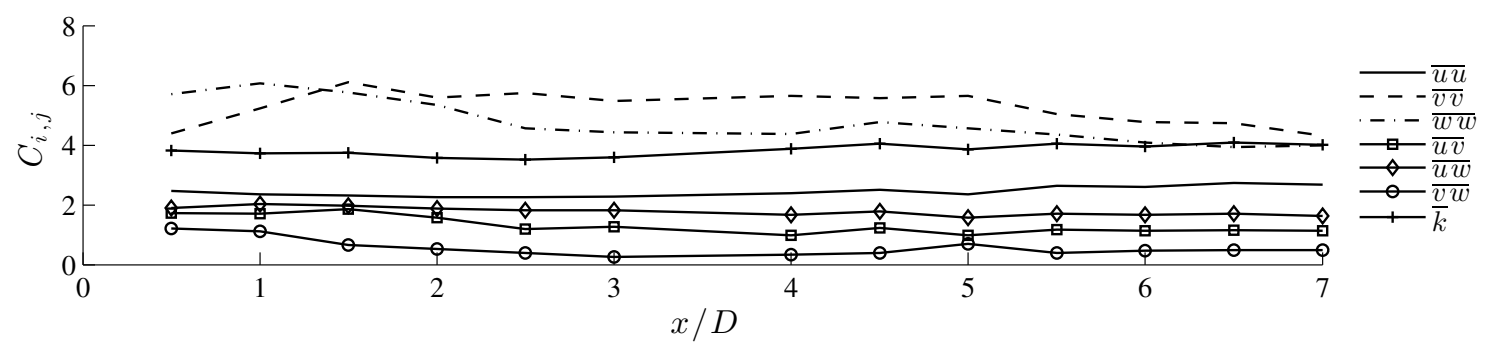

(A) Correction coefficient $C_{i j}$ for each component in the Reynolds stress tensor and TKE versus streamwise coordinate $x / D$.

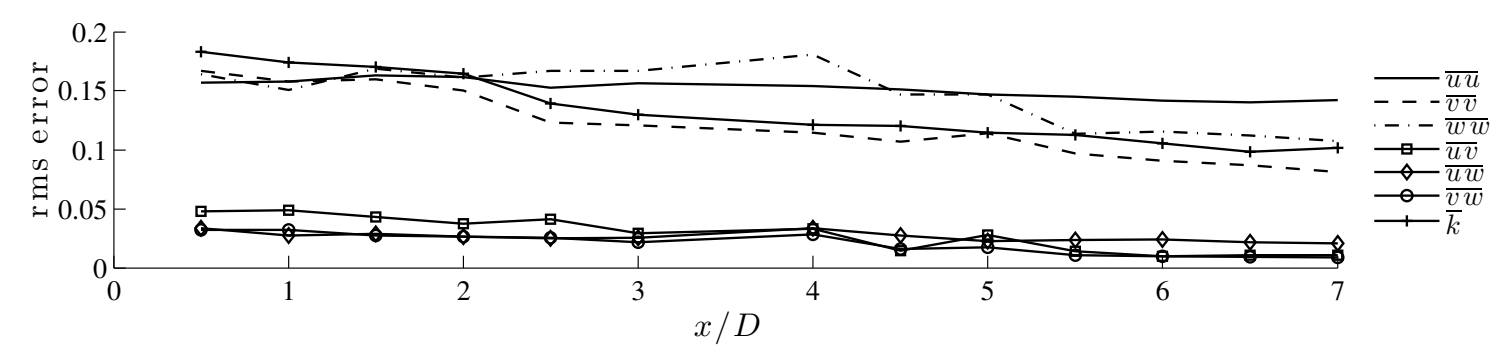

(B) Root-mean-square error associated with the corrected description of stresses versus streamwise coordinate $x / D$.

Figure 4.25: Correction coefficient and associate error for severe reduction (two modes, $N_{r}=2$ ).

are unable to capture the location of peak values along the wall-normal coordinate. At the bottom of the measurement window, the reconstructions are nearly null. A simple correction coefficient is unable to account for this behavior through direct multiplication, and as a result the corrected low-order description underestimates the value of the stresses there.

Representations here do not make use of the DPOD outlined above; each plane requires a separate set of POD modes and eigenvalues to reconstruct the stress field using a total of 26 DOF. The comparisons in figure 4.22 indicate that the stress field can again be simplified by filtering each of the POD bases. To determine at what level the sub-modal filtering should be applied, a coefficient space analogous to that of $C_{i j}$ 
was mapped and analyzed. Minimizing correction error for the span of sub-modes increases the dimension of the coefficient space by one to include POD mode number $n$, downstream location $x / D$, and sub-mode number $m$.

Model correction with sub-modal filtering undertaken here limits the POD basis to only two modes. The coefficient space using sub-modal filtering is not shown here for the sake of space. However, using the full range of sub-modes $m=13$, the coefficients match those of above (figure 4.24) exactly. This matches to expected results as using the full span of sub-modes is exactly equivalent no filtering at all, but requires additional steps in calculation.

Sub-modal filtering applied to the present measurement set showed that the total DOF of the wake could be further reduced without substantial sacrifice of accuracy of the description. Discussed above, the first sub-mode is an effective average of each POD mode in the wake, disregarding the alternation of the algebraic sign in figures 4.13 and 4.14. Accordingly, the eigenvalue associated with the first mode is much larger than for the others. This relationship holds true for the reconstruction amplitudes $b_{n, m}$ as well. These relationships imply that for small $n, \boldsymbol{\Phi}^{(n)}$ may be accurately represented in the entire wake with only a truncated set of corresponding $\Gamma^{(n, m)}$. Filtering with two sub-modes increased the error associated with the corrected reduced-order model by approximately $6 \%$.

As above, the coefficient and associated reconstruction error may be considered approximately constant through the wake. The total simplification of the wake is now from 26,000 degrees of freedom to 4 degrees of freedom (two POD modes filtered to two sub-modes) and a constant. The wake has been simplified to approximately $0.015 \%$ of the original complexity through iterative application of the proper orthogonal decom- 
position. The corrected low-order description represents the normal stresses with accuracy on the order of $12-15 \%$ and shear stresses to within $3 \%$. The coefficient tensor and associated error, respectively, are now,

$$
C_{i j}=\left[\begin{array}{rrr}
2.45 & 1.31 & 1.78 \\
& 5.25 & 0.56 \\
& & 4.75
\end{array}\right], \quad \mathrm{rms}_{i j}(\%)=\left[\begin{array}{rrr}
15 & 2.8 & 2.6 \\
& 12 & 2.0 \\
& & 15
\end{array}\right] .
$$

Figure 4.26 offers a comparison between the original streamwise normal stress derived through Reynolds averaging and that arising from the correction of the low-order description of the wake. For the streamwise normal stress in figure 4.26(B), the obvious difference (and primary source of the error shown in equation (4.6)) is an underestimation in the lower half of the measurement planes for $y / D \leq 1$. Low values of stress in the reconstruction are a related to $\boldsymbol{\Phi}^{(2)}$ having nearly null values below hub-height. Through linear combination, local zeros in POD modes cannot contribute to stresses in the reconstructions. However, trailing the swept area of the blades above hub-height is the region of activity of $\overline{u u}$ and is shown quite clearly in $\left.\frac{0}{u u}\right|_{2} ^{2}$.

Shear stresses reconstruct with approximately 13\% lower RMS error than the diagonal terms of $\frac{\circ}{u_{i} u_{j}}$. Figure 4.27 compares $-\overline{u v}$ and $-\left.\overline{u v}\right|_{2} ^{2}$. This is the component of the Reynolds stress tensor contributing most to the entrainment that drives the recovery of the wake, allows for energy extraction, and resupplies momentum for the wind farm as a whole. The reconstruction $-\left.C_{12} \frac{\circ}{u v}\right|_{2} ^{2}$ represents the expected behavior in the wake to within $2.8 \%$. It is somewhat surprising that a model reduction of this severity is able to reproduce the structures of turbulence in the full wake. The stress field evolves very quickly in the near wake and demonstrates complex contours very near the tur- 


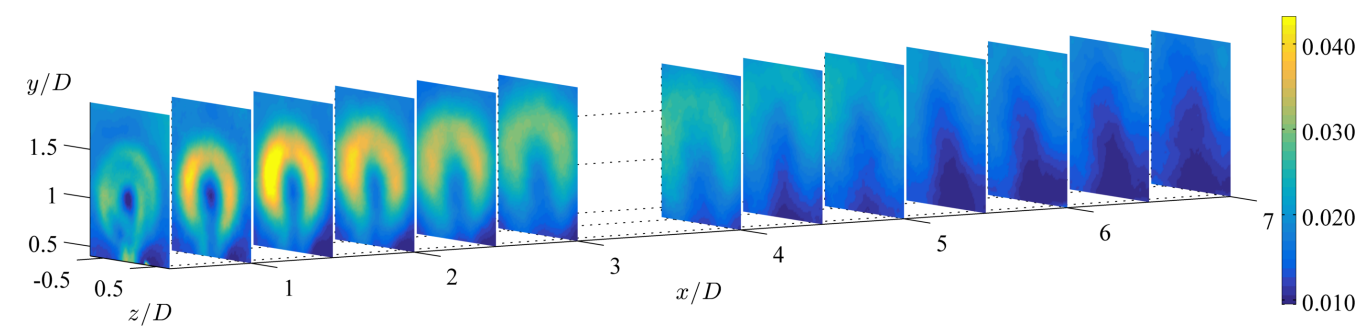

(A) $\overline{u u} / U_{\text {hub }}^{2}$

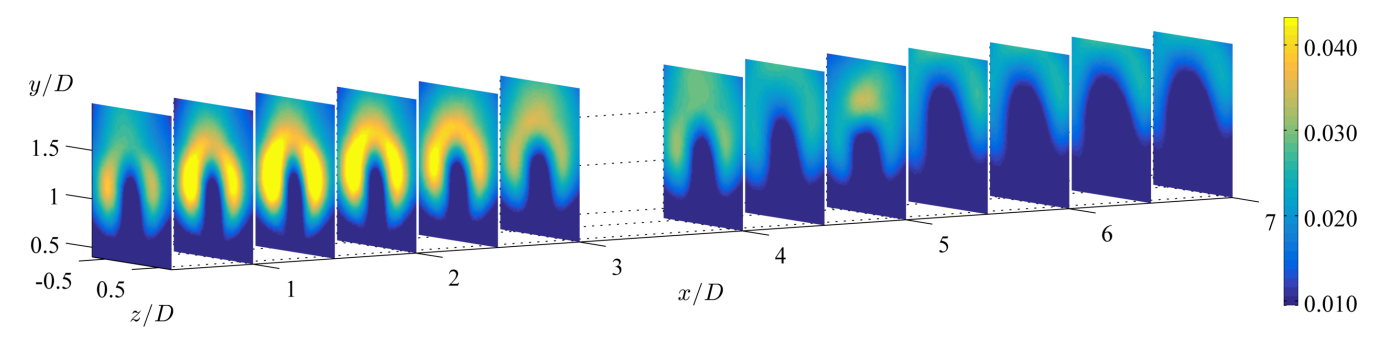

(B) $\left.C_{11} \frac{\circ}{u u}\right|_{2} ^{2} / U_{\text {hub }}^{2}$

Figure 4.26: Comparison of $\overline{u u}$ to $\left.C_{11} \frac{\circ}{u u}\right|_{2} ^{2}$. The modeled normal stress diverges from the statistics most following the mast of the model wind turbine at $z / D=0$.

bine blades. The low-order representation underestimates regions of negative stress trailing the lower half of the turbine rotor. The model also exaggerates the structural complexity of the stress field in the same region. In the far wake $(x / D \geq 4)$, the model appears to impart the wake with greater lateral symmetry than observed in the original statistics. Effects of this nature arise from the symmetry seen in the $\boldsymbol{\Gamma}^{(n, m)}$.

The streamwise-spanwise Reynolds stress $-\overline{u w}$ is reproduced to within $2.6 \%$ in the corrected low-order description. Figure 4.28 confirms the accuracy of the model for $-\overline{u w}$. The error of $2.6 \%$ is largely associated with features directly following the rotor at $x / D=0.5$. As with $-\overline{u v}$, the complexity of the stress field is estimated surprisingly well in the near wake. Fine-scale features are seen quite clearly trailing the top of the swept area of the rotor. In the far wake, the reproduction is quite accurate, showing discrepancies only above the turbine rotor $y / D \geq 1.5$, corresponding with the shear 


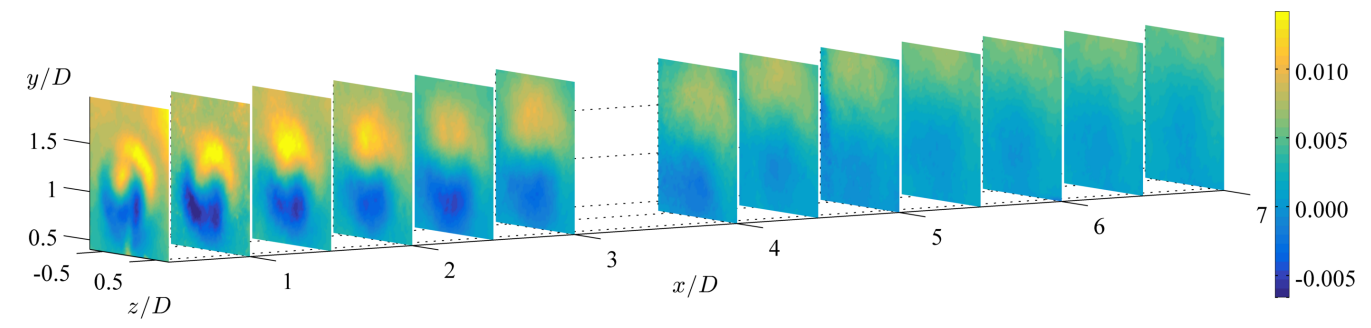

(A) $-\overline{u v} / U_{\text {hub }}^{2}$

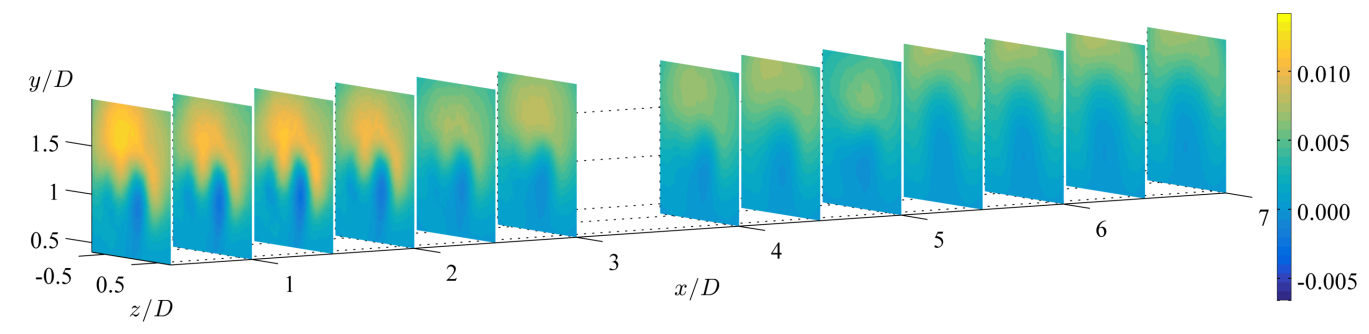

(B) $-\left.C_{12} \frac{\circ}{u v}\right|_{2} ^{2} / U_{\text {hub }}^{2}$

Figure 4.27: Comparison of $-\overline{u v}$ to $-\left.C_{12} \frac{\circ}{u v}\right|_{2} ^{2}$. Error associated with model correction is largely due to the overestimation of $-\overline{u v}$ in the near wake at $x / D \leq 2$.

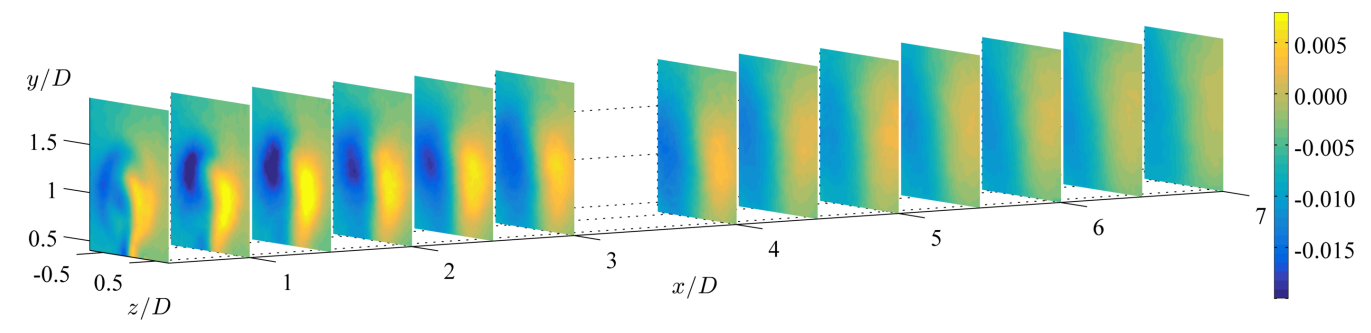

(A) $-\overline{u w} / U_{\text {hub }}^{2}$

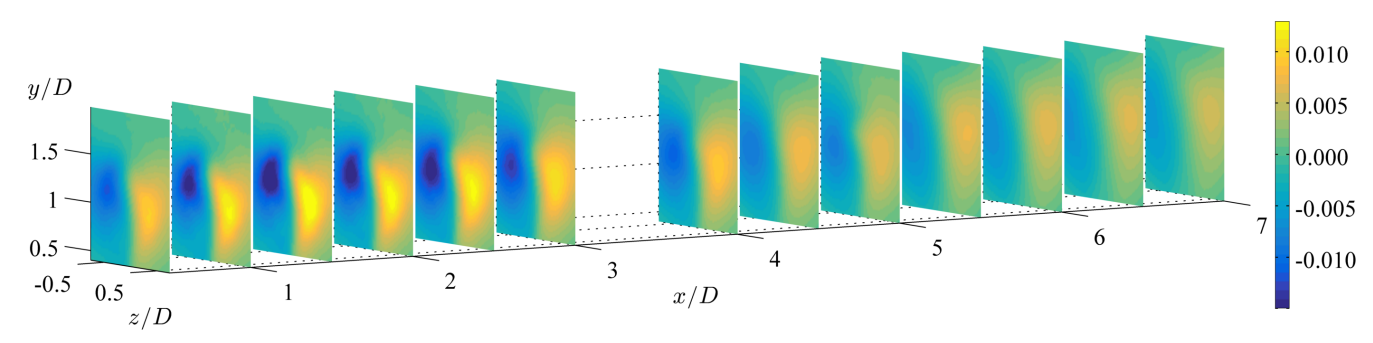

(B) $-\left.C_{13} \frac{\circ}{u w}\right|_{2} ^{2} / U_{\text {hub }}^{2}$

Figure 4.28: Comparison of $-\overline{u w}$ to $-\left.C_{12} \frac{\circ}{u w}\right|_{2} ^{2}$. Features in the near wake $(x / D=$ 0.5 ) are simplified in the low-order description. 
layer that develops between the wake and the flow above the turbine canopy.

\subsubsection{Conclusions}

Wind energy is one path actively being pursued to accommodate the ever-increasing demand for electricity about the globe, which speaks to the necessity of characterizing wind turbine wakes for design of future wind farms. Many models exist already but have historically fallen short of representing the full range of physics observed in experiments and field measurements, especially those mechanisms accounting for the resupply of high-momentum flow from above the canopy into the region where it may be accessed by the turbines Barthelmie et al. (2009); Mortensen and Petersen (1997).

The experiment undertaken accessed the flow via stereo particle image velocimetry resulting in instantaneous velocity measurements with spatial resolution of $1.4 \mathrm{~mm}$ between vectors in 13 planes parallel to the swept area of the rotors. Measurements undertaken here access to the full Reynolds stress tensor and are able to highlight the shear stresses that contribute to the remediation of the wakes. Flow statistics near the wind turbine illustrate the spatially complex distribution of stresses in the wake and the progressively less intense gradients downstream of the turbine.

Applying the snapshot POD to the span of the two-points correlation tensor yields the optimal set of 2000 orthonormal eigenfunctions and eigenvalues that communicate the turbulence kinetic energy. Because the wake of a wind turbine is dominantly defined by the largest and most energetic turbulence structures, the POD is well suited to describing it via truncated bases and low-order descriptions. Decomposition of datasets recorded at different times and locations illuminated POD modes that were clearly related throughout the wake. 
A multi-dimensional application of the proper orthogonal decomposition is developed for the complex flow in the wind turbine canopy. The current approach applies the snapshot POD in time, followed by decomposition about a spatial dimension. Resulting from the double POD are a set of sub-modal structures that span the mathematical space defined by POD mode of common rank throughout the wake. The DPOD as it is formulated here is able to assess the organization of the modes in the wind turbine wake as functions of the streamwise coordinate. Reconstructing the POD bases for each location in the wake is accomplished by linear combination of sub-modes with the set of amplitudes that communicate their respective significance. Application of the POD through the wake transfers dependence on the streamwise coordinate of the wake sub-structures to a set of ordered coefficients. Use of the DPOD to characterize the spatial evolution of energetic POD modes may be extended to other spatially developing flows with little modification.

Rebuilding the Reynolds stress tensor with truncated sets of POD modes necessarily excludes a portion of the turbulence kinetic energy accounted for in the original measurements. It is observed that while a truncated basis consisting of a single mode lacks the complexity necessary to describe the dynamics in the wake, a basis of two modes is able to capture the form, if not the magnitude of the stress field. Based on this observation, a tensor of coefficients was composed to account for excluded energy. The constants were optimized to minimize the root-mean-square error between the original statistics and the low-order description.

Coefficients used to correct the low-order description of turbulence in the wind turbine wake are taken as constants. While the coefficients are maintained as constant, the RMS error in the wake appears to decay linearly with downstream distance from 
the turbine rotor. Analogously, optimizing coefficients to correct the low-order description composed with POD modes filtered at the sub-modal scale further reduced the number of modes required to describe the wake with only a minor loss of accuracy. In this method, the wake was represented with approximately $0.015 \%$ of total degrees of freedom of the original measurement set while maintaining error of $15 \%$ for the normal components of the Reynolds stress tensor and less than $3 \%$ for the shear terms. Considering that the coefficient tensor accounts for energy excluded from the truncated basis, it is hypothesized that a detailed analysis of the turbulence isotropy may provide insight as to the relative magnitude of each correction. The normalized Reynolds stress anisotropy tensor details the balance of energy in the turbulence stress field, providing a physical reasoning for the balance of terms in the correction tensor.

The above analysis demonstrates a method that is applicable to related sets of POD modes derived from SPIV measurements at multiple locations. The correction applied demonstrates that the energy excluded from the low-order description is nearly uniform background energy that influences the magnitude of turbulent stresses but contributes little in the way of discernible features. Influence of the rotor blades is illustrated by the DPOD, which separates the basis of POD modes into an average modal structure and distinct near- and far-wake contributions. That the shear terms in the wake accurately reconstruct with only small corrections suggests that they rely on high-energy, anisotropic turbulence preferentially represented by the POD. Modes that are not coherent in the wake are characterized by a nearly uniform distribution of sub-modal energy. Large-scale structures identified in previous applications of the POD to wind turbine arrays are out of reach in the current measurements, although the streamwise development of roller modes identified by VerHulst and Meneveau (2014) 
would be well-characterized by the DPOD proposed here yielding insight to the entry length of the wind turbine array or the fully developed array condition.

Stress fields composed with the corrected low-order description show surprising complexity in the near wake and evolution of the turbulence field in the wake. Expanding the basis used in the reduced order description allows more subtle features to be expressed in the wake at the cost of overall model reduction. POD modes have been successfully used as the basis functions for predictions derived from the Galerkin Method (see e.g. Kunisch and Volkwein (2002); IEE (2005)). To that end, POD modes are an ideal choice as they are derived from the flow in question and are the optimal basis for representing energy of the flow. sub-modes derived through the DPOD may be combined with Galerkin Projection in the same way, yielding further reduction in the number of basis functions needed. DPOD with correction modeling can simplify the Galerkin approach as the sub-modes exhibit the most characteristic features across the set of POD modes, which should increase model convergence. Alternative reduced order models employing POD modes include the use of artificial neural networks, as employed by Siegel et al. (2008). There neural networks were used as the basis for robust, numerically stable feedback flow control models. The current results are efficiently able to account for the turbulence shear stresses that contribute to bulk flow kinetic energy entrainment into the wake. With a basis of four sub-modal structures, the POD modes may be rebuilt for any point in the wake provided sub-modal amplitudes are available.

In addition to a full order of magnitude further model reduction, the DPOD offers insight beyond the snapshot POD in several ways. The most significant is that the DPOD offers a means of characterizing the spatial development or evolution of 
POD modes. As the POD modes are agnostic to algebraic sign, traditional statistical approaches are difficult to apply. Additionally, the amplitudes $b_{n, m}$ related to the DPOD modes are spatially explicit functions describing the influence of each submodal structure over the span of the DPOD domain. The DPOD not only relates and unifies measurement sets but also filters noise and errors arising from the initial eigenvalue decompositions, leading to cleaner functions to be used in the formation of reduced order models. As an extension of the proper orthogonal decomposition, the DPOD provides a means for further modeling approaches, in particular the modeling of correction terms, which merits further investigation. 


\subsection{Turbulence anisotropy and the proper orthogonal decomposition}

Results pertaining to the example data is reviewed in several stages: a brief review of the turbulence field statistics followed by the corresponding Reynolds stress anisotropy tensor invariant analysis, and the proper orthogonal decomposition. Analytical methods are then combined and discussed in terms of the anisotropy of the turbulence field as represented through truncated POD bases. Finally, several means of correction of the low-order descriptions of the data sets are discussed.

\subsubsection{Turbulence field}

The first SPIV plane discussed is located at one half rotor diameter downstream from the model wind turbine $(x / D=0.5)$ and represents the location of greatest intermittency imparted on the flow by the passage of the rotor blades. At this location, evidence of the rotor is quite clear in each component of the Reynolds stress tensor, seen in figure 4.29. An artifact resulting from a reflection is seen in the area about $(z / D, y / D)=(0.35,0.4)$ in many of the contour plots in the figure.

The Reynolds normal stresses $(\overline{u u}, \overline{v v}$, and $\overline{w w})$ are shown in the diagonal positions of the figure. At this location one observes small-scale features in the flow. Together, they account for the energy described by $k$. All the normal stresses exhibit high magnitudes following the mast of the model turbine. The streamwise normal stress shows peak values tracing the swept area of the roots and tips of the rotor blades. Minimum values of $\overline{u u}$ follow the nacelle of the model turbine. The vertical normal stress $\overline{v v}$ shows an area of high magnitudes combining several effects. Vertical fluctuations

Work from this section has been submitted for publication, Hamilton, N., M. Tutukn, and R. B. Cal. "Anisotropy invariant analysis and the proper orthogonal decomposition of turbulent flows." Physical Review: Fluids. (under review). 

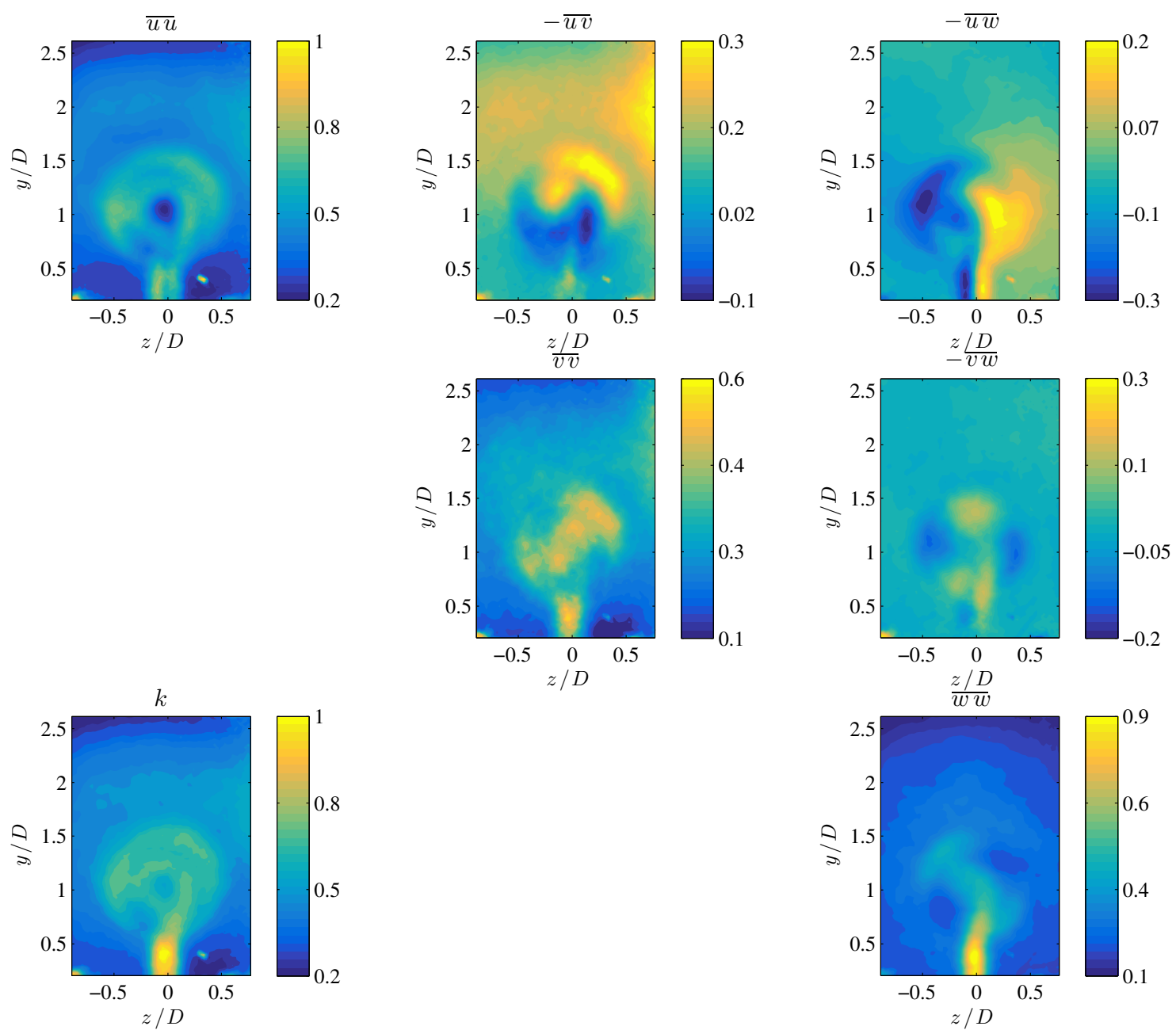

Figure 4.29: Reynolds stresses tensor and $k$ from the wake of a wind turbine at $x / D=0.5$.

in the wake are greatest in intensity issuing from the rotor at top-tip and bottom tip heights and rotated by the bulk flow field. An analogous effect is seen for $\overline{w w}$ where the greatest fluctuations occur at the spanwise extremes of the rotor and are similarly rotated in the wake by the bulk flow.

Asymmetry of the wake arising from the rotating geometry of the wind turbine is evident in the Reynolds shear stresses, especially those including fluctuations of the 
streamwise velocity. As expected from other wind tunnel studies for wind energy (Cal et al., 2010; Chamorro and Porté-Agel, 2011a; Hamilton et al., 2012), positive values of $-\overline{u v}$ occur above hub height in the wake. This stress is associated with the vertical flux of mean flow kinetic energy by turbulence and remediation of the wake. Correlations between the streamwise and spanwise fluctuations of velocity are seen in the contour plot of $-\overline{u w}$ and contribute to lateral fluxes of kinetic energy. Rotation of the turbine rotor influences $-\overline{u v}$ and $-\overline{u w}$ similar to the normal stresses discussed above. The inplane Reynolds shear stress $-\overline{v w}$ is approximately symmetrical about the hub in both the $x y$ - and $x z$-planes.

In the bottom left corner of figure 4.29 is a contour plot of the turbulence kinetic energy. It is unsurprising that the dominant features of $k$ correspond with those of $\overline{u u}$, as it is the dominant component of the Reynolds stress tensor for the flow in question. The turbulence kinetic energy is included in the figure for its theoretical contribution to the analysis methods below; turbulence kinetic energy integrated over the measurement domain is reflected by the POD eigenvalues and it is also used to normalize the Reynolds stress tensor for the anisotropy analysis below.

A measurement plane from the far wake was selected located at $x / D=6$ as the turbulence exhibits different behavior here than near the model wind turbine. At this location the wake is largely recovered and the flow is well-mixed. Each of the turbulent stresses is more uniformly distributed in the measurement plane and has decreased in magnitude from the previous examples. Evidence of rotation is almost completely absent from the normal stresses with the exception of $\overline{u u}$, which continues to demonstrate some slight asymmetry.

The magnitudes of the shear stresses are greatly reduced compared to their previ- 

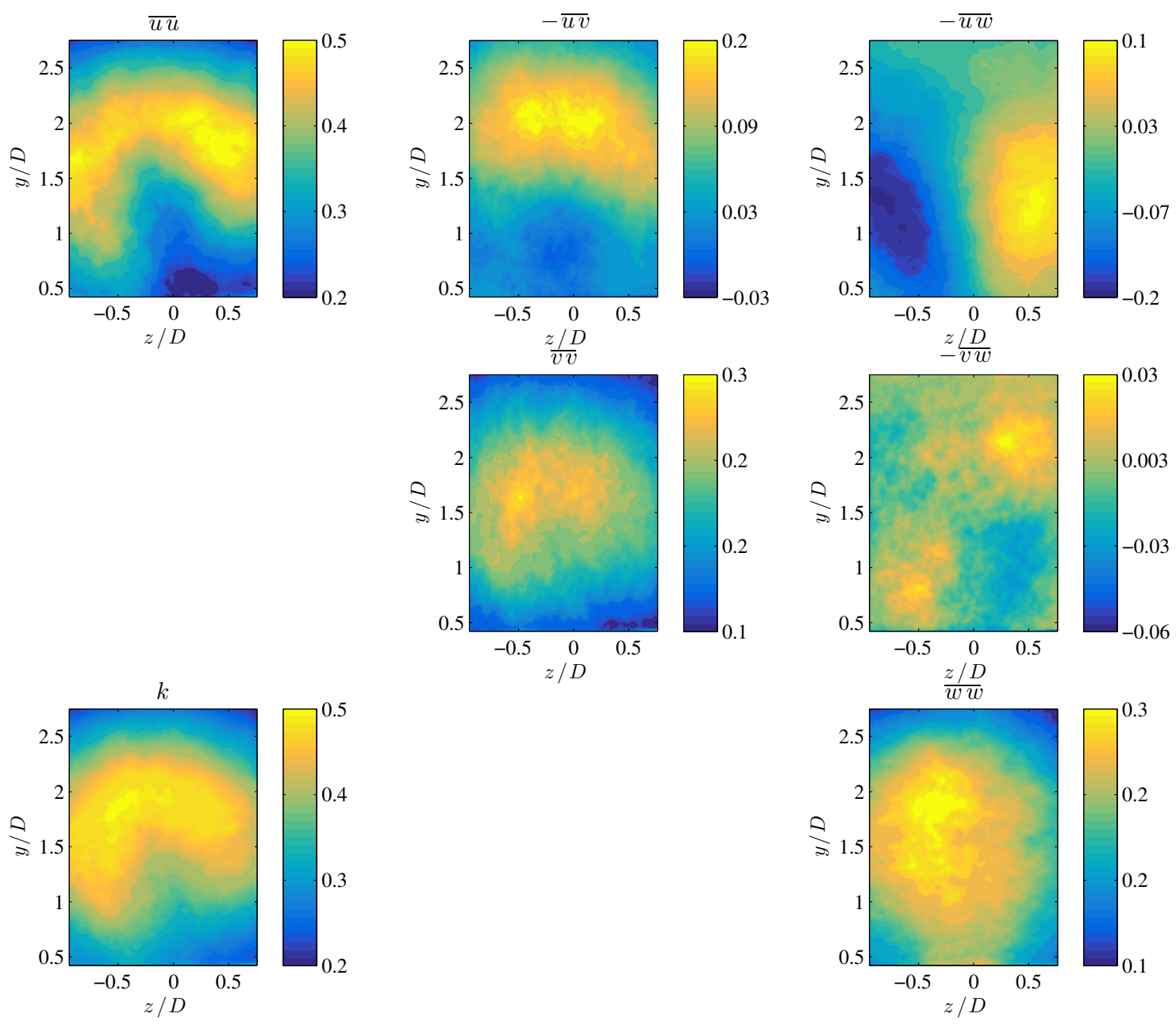

Figure 4.30: Reynolds stresses tensor and $k$ from the wake of a wind turbine at $x / D=6$.

ous values. Those stresses contributing to the flux of kinetic energy $(-\overline{u v}$ and $-\overline{u w})$ demonstrate magnitudes less than $50 \%$ of their corresponding near-wake values, indicating that the turbulence field is fairly uniform at this point in the wake. The in-plane stress $-\overline{v w}$ has reduced in magnitude to approximately $10 \%$ of its former values, although it retains the features seen throughout the wake. Although they differ slightly in magnitudes, each of the normal stresses demonstrate that the flow tends toward 
homogeneity far into the wake. As the shear terms fall off, one may also consider that the normal terms become more representative of the principle stresses. This tendency toward uniformity is characteristic of well-mixed turbulence and is reflected in the invariants of the normalized Reynolds stress anisotropy tensor below.

Data from the DNS of the fully developed channel flow is seen in figure 4.31. The data presented in the figure follows the same arrangement as the above figures for the wind turbine wake. The data presented is a small subset of the total data from the simulation, accounting for decreased convergence of turbulence statistics. The data included here was intentionally down sampled, both spatially and temporally, for the purposes of low-order representation. Regardless of downsampling, the characteristic features of the turbulence close to the wall on one side of the channel are represented in the contours in figure 4.31 .

Stresses presented for the channel flow differ from those of the wind turbine wake; the spatial organization of energy present in each component of the stress tensor reflects the influence of the wall on the flow. Direct numerical simulation undertaken here is the product of extensive technical development such that the resulting turbulence field matches boundary conditions derived theoretically and observed in closely controlled experiments. The inner boundary layer is clearly seen in the simulation results as minimum values of all components of the Reynolds stress tensor. Profiles of the stress field are seen in the associated documentation (Graham et al., 2013) with greater statistical convergence.

The simulation data reflects the wall boundary conditions as null values of each Reynolds stress at the wall. Immediately above the wall, stresses and turbulence kinetic energy take on non-null values. The inner boundary layer is evidenced as the 

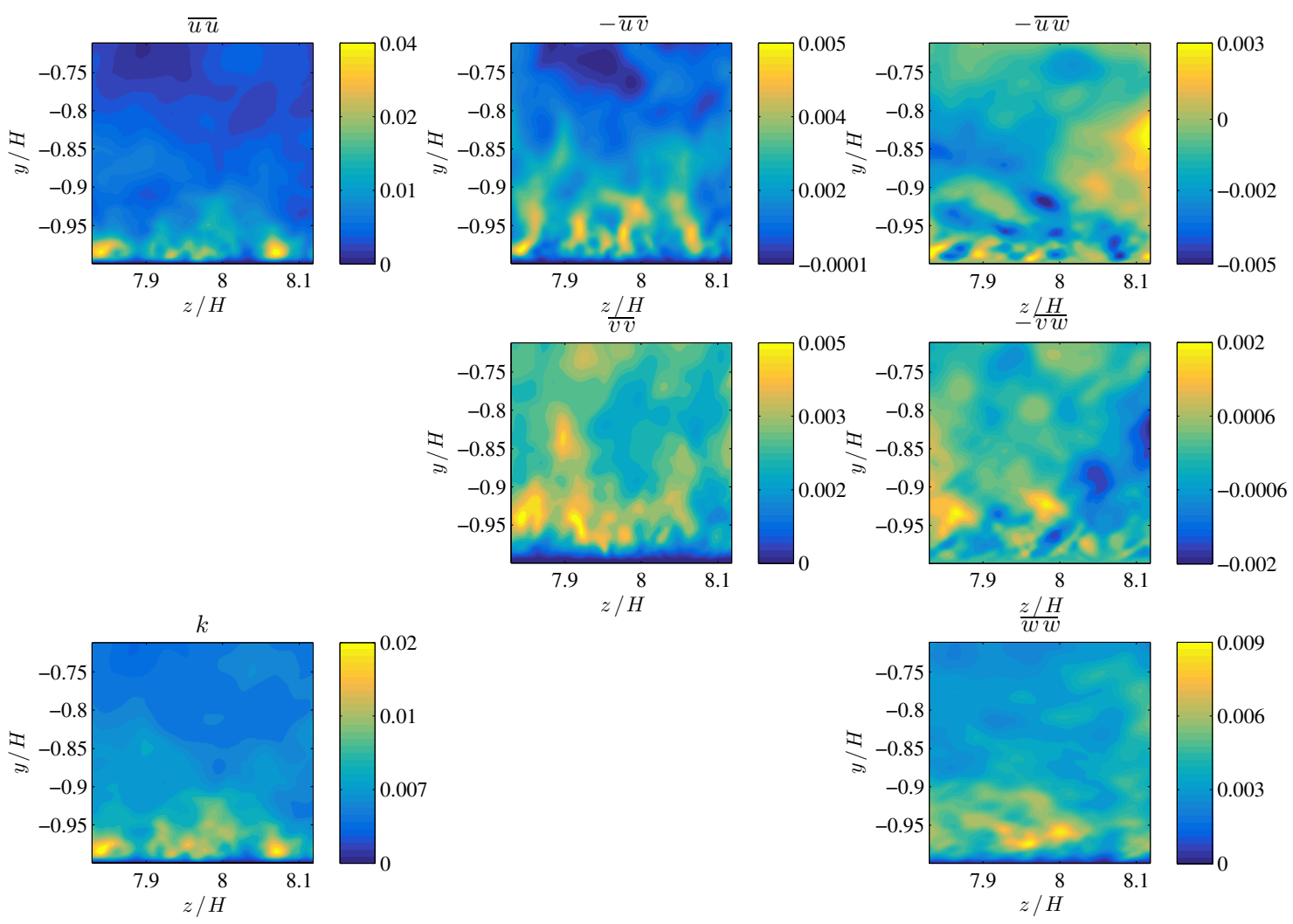

Figure 4.31: Reynolds stresses tensor and $k$ from the fully developed channel flow DNS.

region where viscous forces dominate and the resulting turbulence is low in magnitude. Magnitudes of turbulence stresses increase quickly with $y^{+}$; the streamwise normal Reynolds stress and $k$ show peak values at $y^{+}=16.5(y / H \approx-0.9835)$. Maximum values of $\overline{v v}$ and $\overline{v v}$ occur further from the wall. Shear terms are lower in magnitude than the normal stresses and take on negative values in the flow. All stresses from the DNS channel flow are non-dimensionalized by the channel half-height $H$, and the wall shear velocity $u_{\tau}=0.0499$. The DNS was performed with non-dimensional values, and as a result each component of $\overline{u_{i} u_{j}}$ demonstrates values approximately two orders of magnitude lower than in the wake of the wind turbine seen above. In the following re- 
view of the anisotropy tensor invariants, it is clear that the anisotropy a turbulent flow is dependent the deviation from isotropic turbulence rather than the magnitudes of the Reynolds stress tensor.

\subsubsection{Reynolds stress anisotropy}

Invariants of the selected measurement planes are shown in figure 4.32. Agreeing with the Reynolds stresses above, the invariants demonstrate a decrease in spatial organization moving downstream from the model wind turbine. Subfigures correspond to $x / D=0.5$ in figure 4.32(A), $x / D=6$ in figure 4.32(B), and the channel flow in figure 4.32(C). Contours of $\eta$ from the near wake (figure 4.32(A)) indicate that the minimum values occur trailing the nacelle of the turbine close to the device. Increased $\eta$ indicates a higher degree of anisotropy in the turbulence. Maxima of $\eta \approx 0.22$ occur at the spanwise borders of the wake $(z / D \approx \pm 0.5)$ and in the upper corners of the measurement plane. By $x / D=6$ (figure 4.32(B)), large-scale mixing in the wake increases the uniformity of the turbulence field. Downstream from the wind turbine, turbulence decays and becomes increasingly homogeneous and tends toward isotropy. Accordingly, the second invariant is smaller than in the near wake, indicating that the turbulence tends toward isotropy moving away from the turbine.

The third invariant $\xi$ delineates whether the turbulence field is well represented by a single dominant component $(\xi>0)$ or two co-dominant components $(\xi<0)$. Near the turbine $(x / D=0.5)$, the third invariant shows a region of $\xi<0$ following the mast and the lower part of the rotor area. As with the turbulent stresses, the region of negative $\xi$ is made asymmetric by rotation of the bulk flow. In the far wake $(x / D=6)$, $\xi$ is symmetrically distributed in the wake as effects of rotation are largely gone from 
the flow. The magnitude of $\xi$ is reduced in the far wake following the transition of the turbulence toward homogeneity. As with $\eta$, increasingly isotropic flow requires small magnitudes of $\xi$.

Lumley triangles are shown for the SPIV measurement planes in the right column of figure 4.32. Data for the near wake shows that the turbulence occupies a large region of the anisotropy invariant space. Interesting to note is that $\xi$ is always either significantly positive or significantly negative; the center of the Lumley triangle is not occupied by the invariants for $x / D=0.5$. Another noteworthy observation for the wind turbine wake is that the turbulence tends toward positive $\xi$, indicating that the turbulence is dominated by a single large principle stress for much of the wake. Further downstream, the turbulence is much more isotropic as indicated by the occupation of the lower region of the Lumley triangle at $x / D=6$, although it never reaches the perfectly isotropic condition, where $\eta=\xi=0$.

Invariants of the channel flow show different behavior than the wind turbine wake following the nature of the flow. Differences are clearly seen in the near-wall region $y / H<-0.95$, where the magnitudes of both invariants are quite large. This region conforms to boundary conditions imposed on the flow during simulation. To match wall-bounded flow behavior, the stresses peak near the wall. In the viscous sublayer $\left(y^{+} \approx 10\right)$, nearly all turbulence is suppressed. Immediately above the wall, the only non-null Reynolds stress is $\overline{u u}$, there leading to data with identically one-dimensional turbulence $(\eta=\xi=1 / 3)$. With increasing wall-normal distance, the spanwise normal stress begins to emerge and the turbulence follows the two-component boundary of the Lumley triangle. Increasing $y / H$, the remaining Reynolds stresses express energy and the invariants shift suddenly to three-dimensional turbulence. In the outer re- 

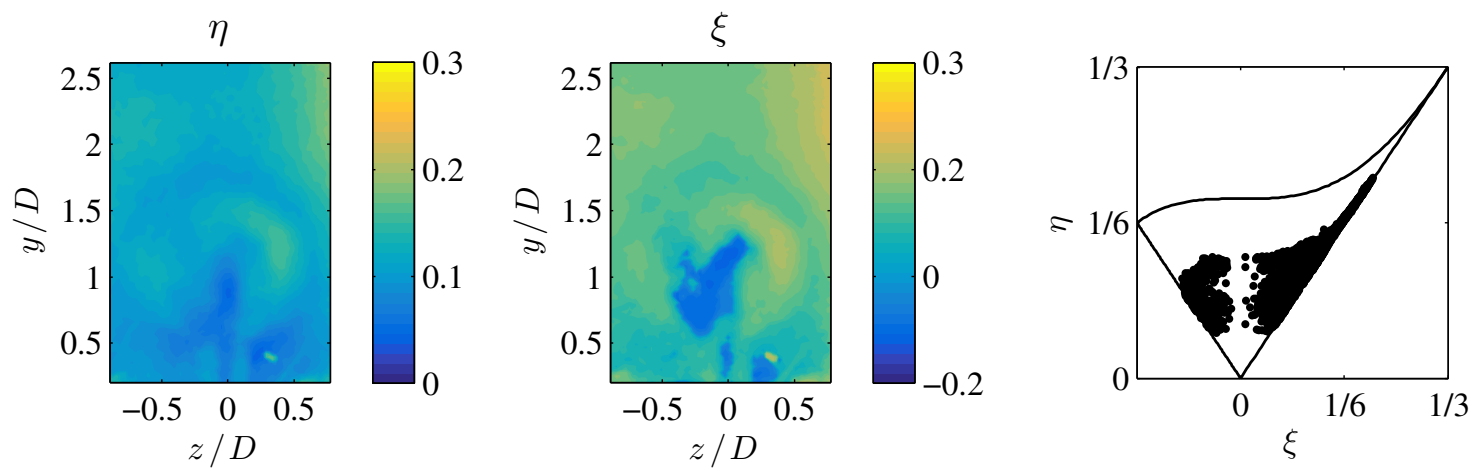

(A) $x / D=0.5$
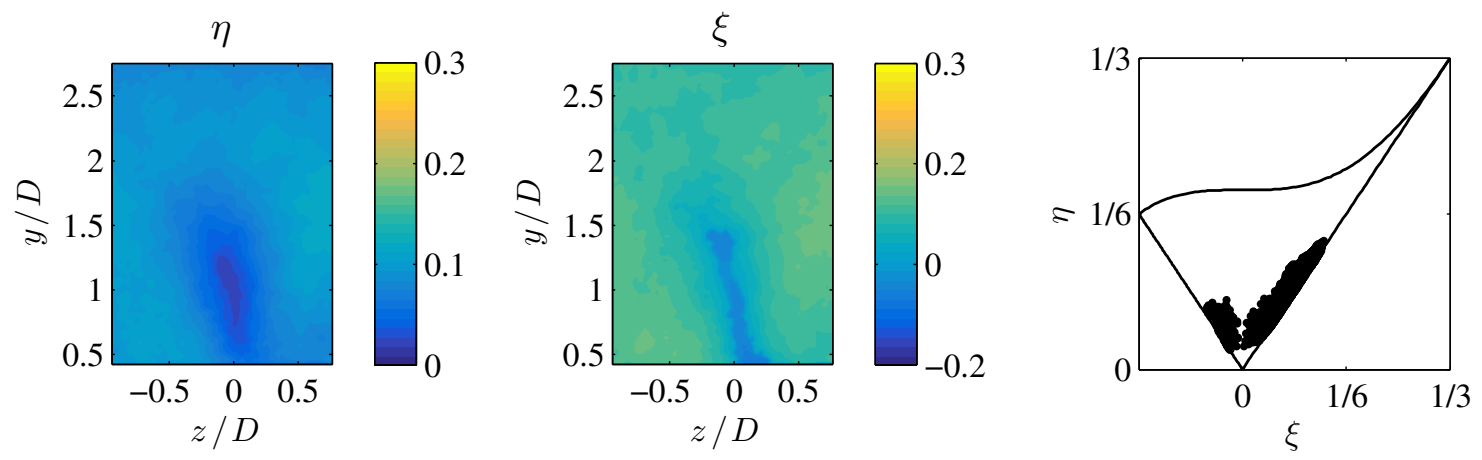

(B) $x / D=6$
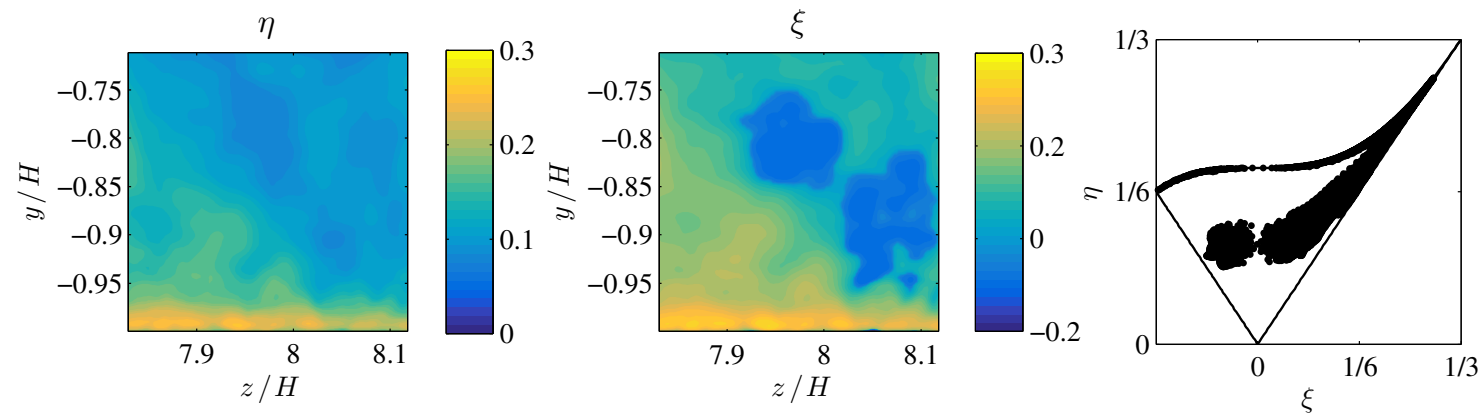

(C) Channel flow DNS

Figure 4.32: Anisotropy tensor invariants and corresponding Lumley triangles for included data.

gion of the boundary layer $\left(y^{+} \geq 50\right)$, the turbulence is less organized in the sense of the anisotropy tensor invariants; meaning, the second invariant spans $0.1 \leq \eta \leq 0.3$ 
and the third invariant spans $-0.1 \leq \xi \leq 0.3$. The turbulence in the outer region of the channel flow (not shown) is, in an ensemble sense, more isotropic than the log layer. Moving away from the channel wall follows the trends described by Rotta (1951) and Pope (2000) wherein the invariants tend toward zero with increasing $y / H$ and turbulence becomes more isotropic.

\subsubsection{Snapshot POD results}

The two selected measurement planes from the wind turbine wake each have 2000 POD modes corresponding to the 2000 velocity snapshots used to formulate the kernel of the POD. Each mode is also associated with an eigenvalue that communicates the energy associated with that mode throughout the measurement set. Similarly, the channel flow data has 1180 POD modes issuing from the snapshots sampled from the simulation data. Normalized eigenvalues from the POD for each dataset are seen in figure $4.33(\mathrm{~A})$.

One of the major benefits of the POD arises from its ability to sort dominant features of the flow toward the front of the resulting modal basis, allowing the dominant features of the turbulence field to be represented with very few modes. Figure 4.33(B) shows the cumulative summation of the eigenvalues from each dataset compared to frequently used thresholds. The point of truncation of a POD mode basis is frequently arbitrary, often taking a threshold of a given portion of the total energy expressed by the eigenvalues of the POD based on the assumption that the main features of the flow are captured by the first few modes. Thresholds of these sort are seen in figure 4.33(B) as gray horizontal lines. Reconstructing the Reynolds stress tensor with a truncated set of POD modes typically describes the important features of the turbulence but neces- 
sarily excludes energy from the description. For example, a threshold of $50 \%$ energy requires very few modes $(8,13$, and 18 modes for the channel flow, wake at $x / D=6$, and wake at $x / D=1.5$, respectively) but omits energy from the majority of modes. Intermediate and high modes are taken to describe small scales of turbulence that are relatively isotropic and contribute little energy to the turbulence field. Gray lines in figure 4.33 (B) correspond to 50, 75, and 90\% thresholds of energy designated by the POD eigenvalues.

The flows are easily distinguished by the trends shown in figure 4.33(B). POD eigenvalues spectra from wake data indicate that many more modes are required to recover the full range of dynamics in the flow. Trends for $x / D=0.5$ and $x / D=6$ in the solid and dashed lines are flatter than for the channel flow, indicating that there is a broader range of energetic structures in the wake. In contrast, the channel flow data accumulates energy with few modes. Nearly all of the energy is present in the first 100 modes and the remaining basis describes very little. This is due in part to limiting the range of the sampled data to exclude the outer portion of the domain. In wall-bounded flows, the range of length scales observed is a function of wall-normal distance. Performing a similar POD over the channel half-height yields a greater range of POD modes describing energetic structures in the flow. The rapid accumulation of energy is still seen in across $H$ compared to the wake data, as is the flat region of the eigenvalue spectrum for $N_{r} \geq 10^{2}$.

In figure 4.33(A), energy associated with each POD mode is normalized by the turbulence kinetic energy integrated over each measurement domain. Each normalized eigenvalue of the POD describes the relative importance of its respective POD mode to the turbulence field. The distribution of energy in the normalized eigenvalues for 


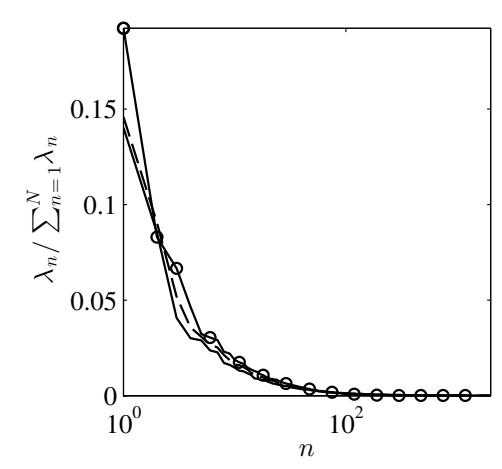

(A) Normalized eigenvalues from POD of WTA and channel flow.

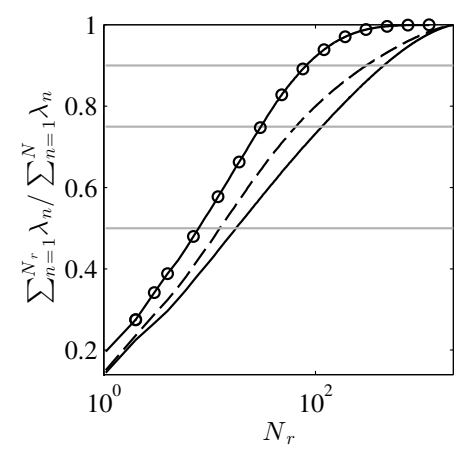

(B) Normalized cumulative summation of eigenvalues.

Figure 4.33: Eigenvalues from the snapshot POD for the wind turbine wake at $x / D=0.5$ (solid black lines), $x / D=6$ (dashed lines), and the channel flow DNS data (circles).

the wake measurements (solid and dashed lines) are nearly identical to one another, arising from the similarity in POD modes throughout the wake. Hamilton et al. (2016) demonstrated that POD modes are subject to streamwise evolution throughout the wake. Eigenvalues for the channel flow (indicated with circles) fall off more quickly than for the wake. The concentration of energy in few eigenvalues suggests that energy is contained in a few coherent structures that exist in the channel flow boundary layer.

In low-order descriptions, the POD basis is separated into isotropic and anisotropic portions analogous to decomposing the turbulence field according to equation (2.10). The isotropic portion of the field is assumed to be accounted for by the small scales, represented by intermediate and high-rank POD modes. The anisotropic contribution to the total turbulence field, is represented by the lowest ranking POD modes and most energetic structures. The POD eigenvalues delineate the turbulence kinetic energy expressed by the Reynolds stress tensor integrated over the domain, equal to the sum of 
the isotropic and anisotropic turbulence,

$$
\sum_{n=1}^{N} \lambda^{(n)}=\int_{\Omega} \hat{k} d \Omega+\int_{\Omega} \grave{k} d \Omega .
$$

In equation (4.7) and in the following analysis, quantities designated with an over-ring (e.g. $\stackrel{\circ}{k}$ ) represent the anisotropic turbulence described with the low-rank POD modes. The complimentary isotropic contributions are designated with the caret (e.g. $\hat{k}$ ), and are composed of the remaining POD modes. The majority of turbulence structures are considered to be part of the isotropic turbulence field, including contributions from intermediate and high-rank POD modes.

The Reynolds stress tensor is represented with a truncated mode basis according to,

$$
\frac{\circ}{u_{i} u_{j}}=\sum_{n=1}^{N_{r}} \lambda^{(n)} \phi_{i}^{(n)} \phi_{j}^{(n)}
$$

With the low-order representation of the Reynolds stress tensor calculated according to equation (4.8), the anisotropic turbulence kinetic energy is written $\stackrel{\circ}{k}=$ $\frac{1}{2}\left(\frac{\circ}{u u}+\frac{\circ}{v v}+\frac{\circ}{w w}\right)$. In the same sense, the isotropic contributions to the turbulence field may be represented with equation (4.19) from the point of truncation $N_{r}$ to the end of the basis,

$$
\hat{\overline{u_{i} u_{j}}}=\sum_{n=N_{r}+1}^{N} \lambda^{(n)} \phi_{i}^{(n)} \phi_{j}^{(n)} .
$$

The analogous turbulence kinetic energy composed with isotropic contributions is $\hat{k}=\frac{1}{2}(\hat{u u}+\hat{v v}+\hat{w w})$. Representations of the turbulence field with the anisotropic or isotropic modes are seen in the following figures. In the each reconstruction, $50 \%$ of 
the total turbulence kinetic energy is included in the low-order descriptions according to the cumulative summation of $\lambda^{(n)}$ seen in figure 4.33(B). A division at this point imposes the balance $\int \stackrel{\circ}{k} d \Omega=\int \hat{k} d \Omega$.

For brevity, only components of the Reynolds stress tensor including fluctuations of the streamwise velocity have been included. In the contour plots in figure 4.34, one observes that while many of the distinctive features seen in the full stress field at $x / D=0.5$ are represented by $\frac{\circ}{u_{i} u_{j}}$, the magnitudes of each stress are reduced. The streamwise normal stress $\frac{\circ}{u u}$ exhibits the azimuthal streaks resulting from passage of the rotor blades seen in the full statistical values. However, the isotropic portion $\hat{u u}$ shows no evidence of rotation in the flow. Instead, the isotropic part is nearly uniform in the swept area of the rotor. Similar behavior is seen in both of the shear stresses in figure 4.34. Both $\frac{\circ}{u v}$ and $\frac{\circ}{u w}$ show characteristic regions of positive and negative magnitudes and the effects of bulk rotation discussed above. Notably, the isotropic contributions to the shear stresses are nearly null everywhere except directly following the wind turbine model. In the wake area, the stresses show magnitudes on the order of $20 \%$ of their original statistical values. The reflection seen in the statistics is captured by the isotropic portion of the turbulence, indicating that the reduced order description using the anisotropic modes acts as a filter for the turbulence statistics.

Low-order representation recovers dominant flow features in the far wake equally well. At $x / D=6$, the salient features of the stresses are all present in $\frac{\circ}{u_{i} u_{j}}$, confirmed by the uniformity of each of the isotropic contributions. Comparing $\overline{u_{i} u_{j}}$ to $\overline{u_{i} u_{j}}$ from figure 4.30, the behavior is accounted for by the anisotropic contribution. Magnitudes of the contours in 4.35 (B) are approximately $80 \%$ of the original statistical values. Even more than in the near wake, the isotropic contributions are uniform and small for 

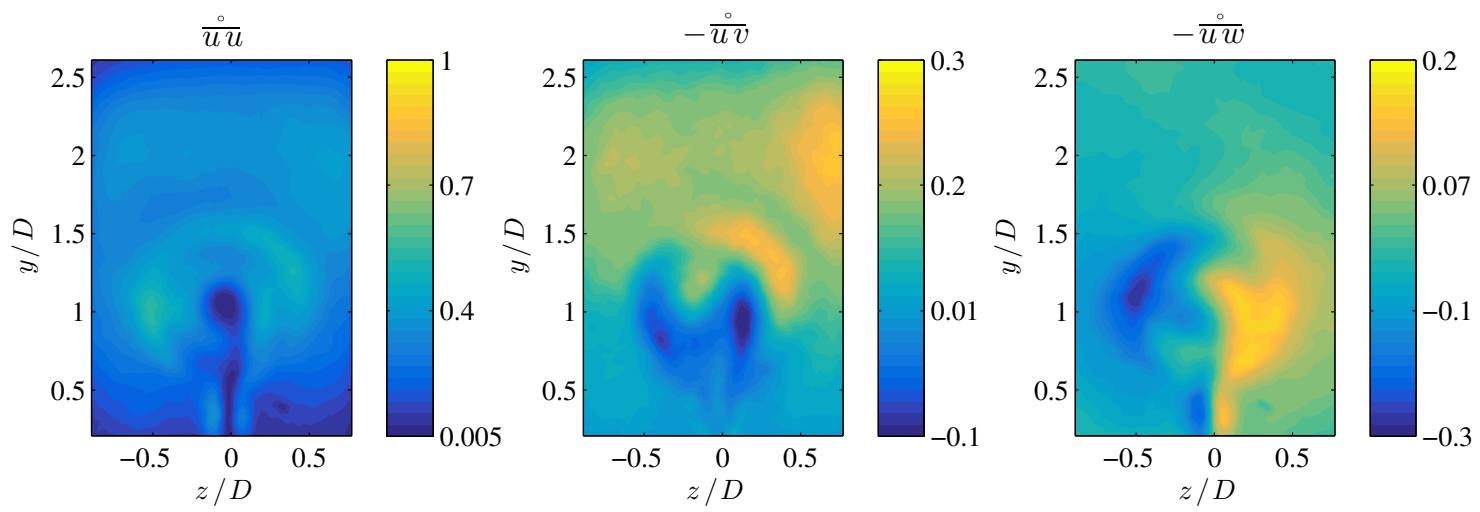

(A) $\frac{\circ}{u_{i} u_{j}}$ at $x / D=0.5$.
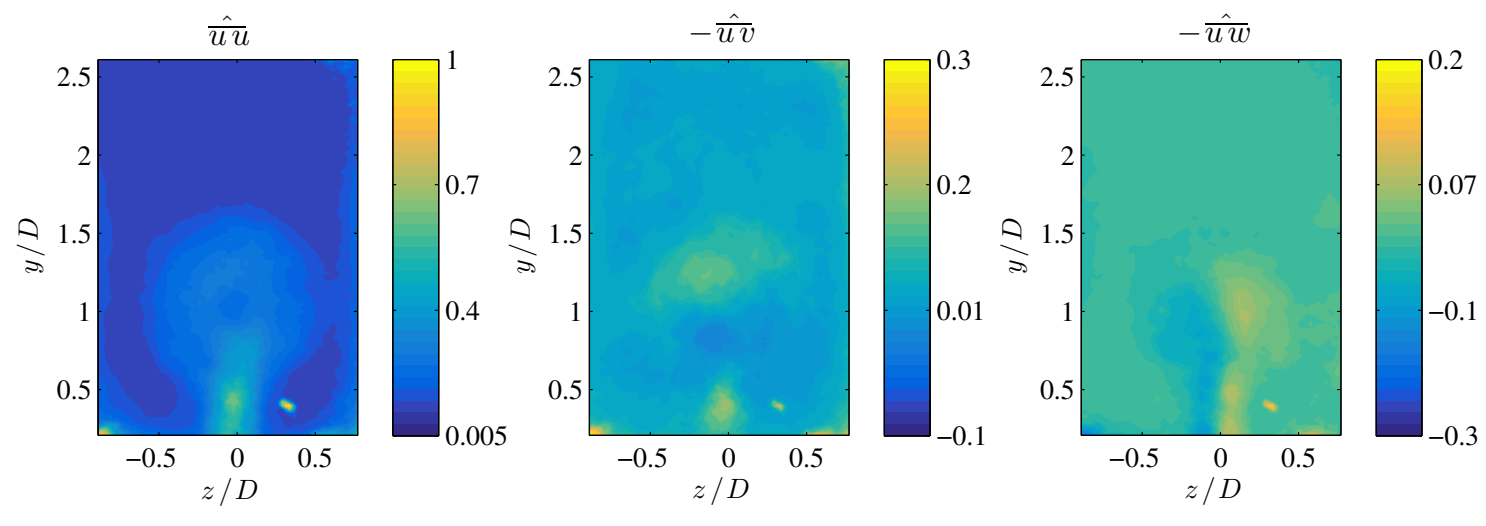

(B) $\hat{u_{i} u_{j}}$ at $x / D=0.5$

Figure 4.34: Anisotropic (top) and isotropic (bottom) contributions to selected turbulence statistics. At $x / D=0.5,50 \%$ energy requires $N_{r}=18$ modes.

$x / D=6$. There, the normal stress $\hat{u u}$ approximately $10^{-1} \mathrm{~m}^{2} / \mathrm{s}^{2}$ and the shear terms are on the order of $10^{-2} \mathrm{~m}^{2} / \mathrm{s}^{2}$.

The channel flow turbulence is divided into isotropic and anisotropic portions, seen in figure 4.36. In the turbulence represented by the anisotropic range of the POD basis, most of the energy is present in the contours as well as the large scale features of the flow. Visible for the channel flow is that the anisotropic portion is not capable of capturing all of the near-wall behavior. The normal stress $\frac{\circ}{u u}$ is nearly equal 

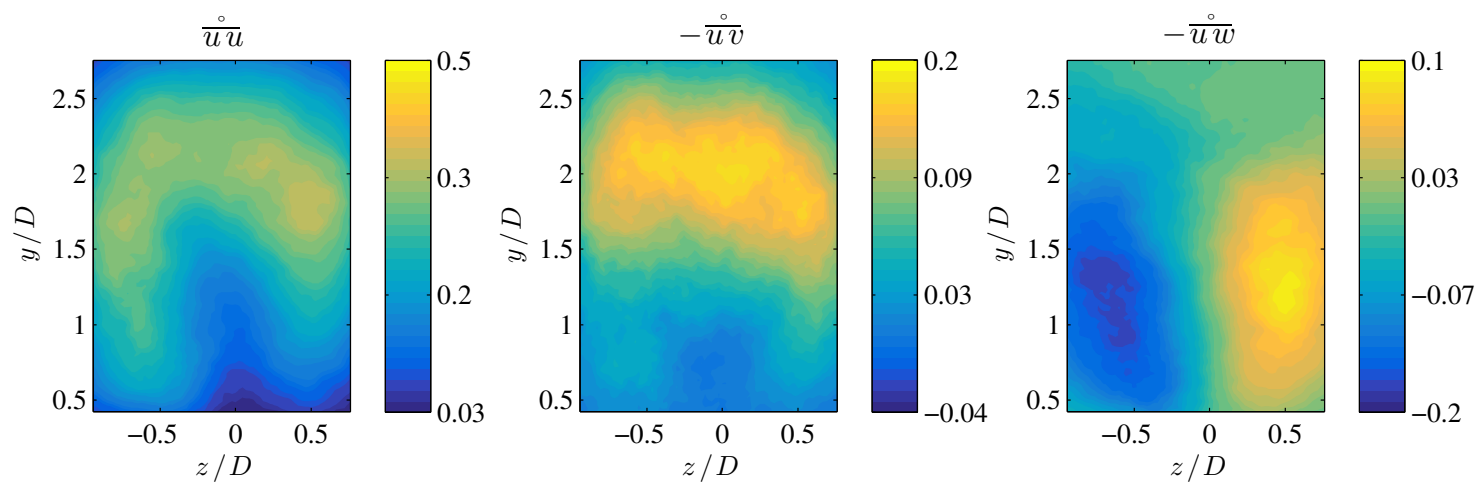

(A) $\frac{\circ}{u_{i} u_{j}}$ at $x / D=6$.
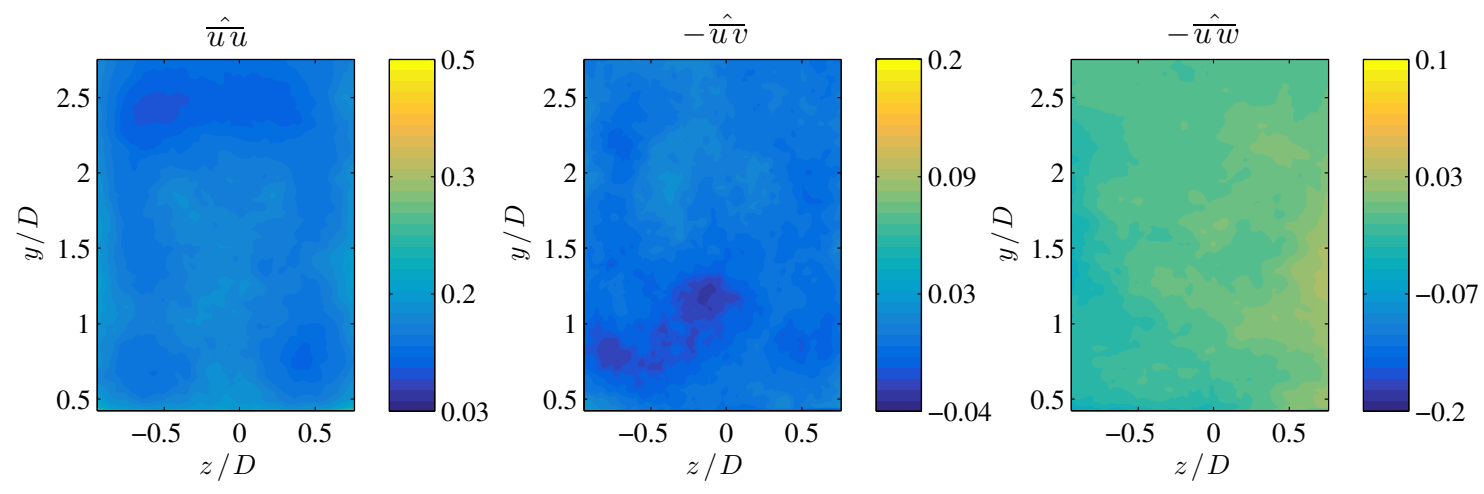

(B) $\hat{u_{i} u_{j}}$ at $x / D=6$

Figure 4.35: Anisotropic (top) and isotropic (bottom) contributions to selected turbulence statistics. At $x / D=6,50 \%$ energy requires $N_{r}=13$ modes.

in magnitude to the isotropic part $\hat{u u}$ close to the wall. Like the wind turbine wake in figures 4.34 and 4.35 , the anisotropic shear stresses in the channel flow are nearly identical to the description using the full statistics. Interestingly, the shear terms are not equally represented. The streamwise-spanwise stress $\frac{0}{u w}$ appears to be slightly overestimating the shear close to the wall. This leads to unexpected behavior in terms of the anisotropy tensor invariants discussed below. Far from the wall ( $y / H \geq-0.9)$, the isotropic contribution is uniformly distributed and contributes little in the way of flow features that $\frac{\circ}{u_{i} u_{j}}$ does not account for. Trends seen in figure 4.36 are consistent 

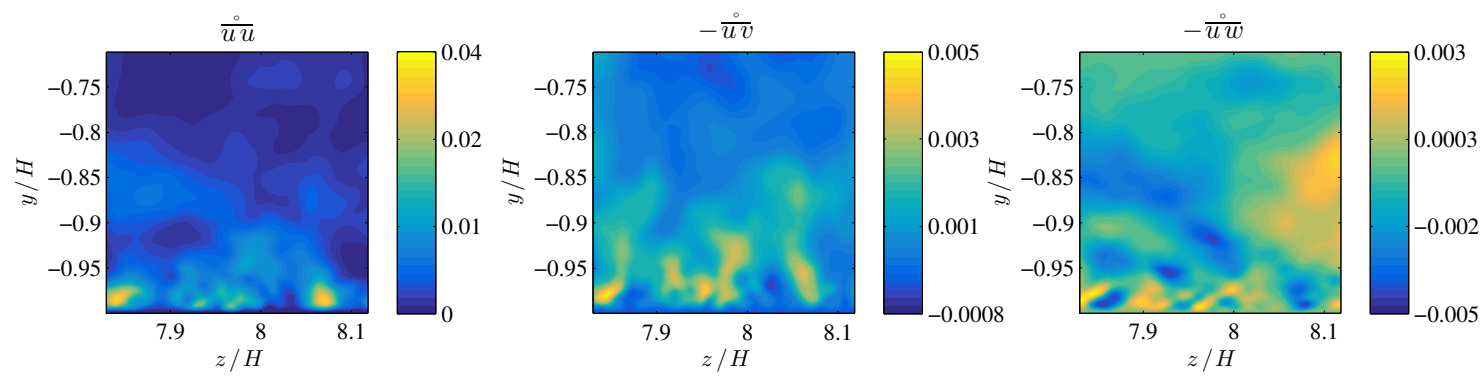

(A) $\frac{\circ}{u_{i} u_{j}}$ from the channel flow.
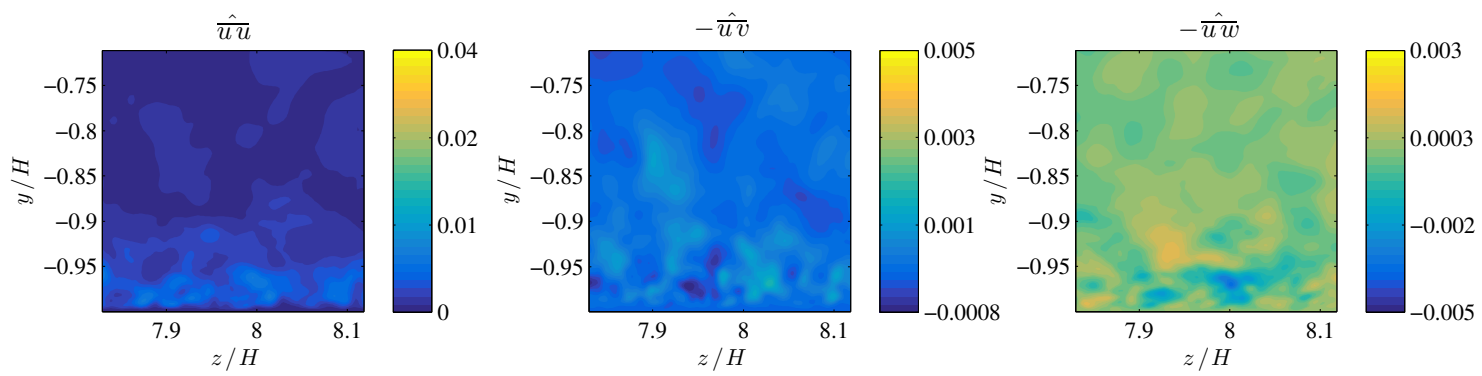

(B) $\hat{\overline{u_{i} u_{j}}}$ from the channel flow.

Figure 4.36: Anisotropic (top) and isotropic (bottom) contributions to selected turbulence statistics. For the channel flow, 50\% energy requires $N_{r}=8$ modes.

in the channel half-height. In such a case, the number of modes required to reach $50 \%$ energy is greater and the contours of $\frac{\circ}{u_{i} u_{j}}$ are more similar to the full Reynolds stress tensor. The contribution made by $\hat{u_{i} u_{j}}$ is reduced and tends toward zero with wallnormal coordinate. Nearly all of the features of the turbulence in the central region of the channel are accounted for by $\frac{\circ}{u_{i} u_{j}}$.

Another means of gauging the quality of POD reconstructions is to compose reduced order Reynolds stress anisotropy tensors and to compare their invariants as in equations (2.12) and (2.13). The resulting invariants reveal much about the character of the flow not immediately visible in contours of the stresses. Figure 4.37 offers a comparison between the AIMs of the invariants of $b_{i j}, \stackrel{\circ}{b}_{i j}$, and $\hat{b}_{i j}$, from left to right, 

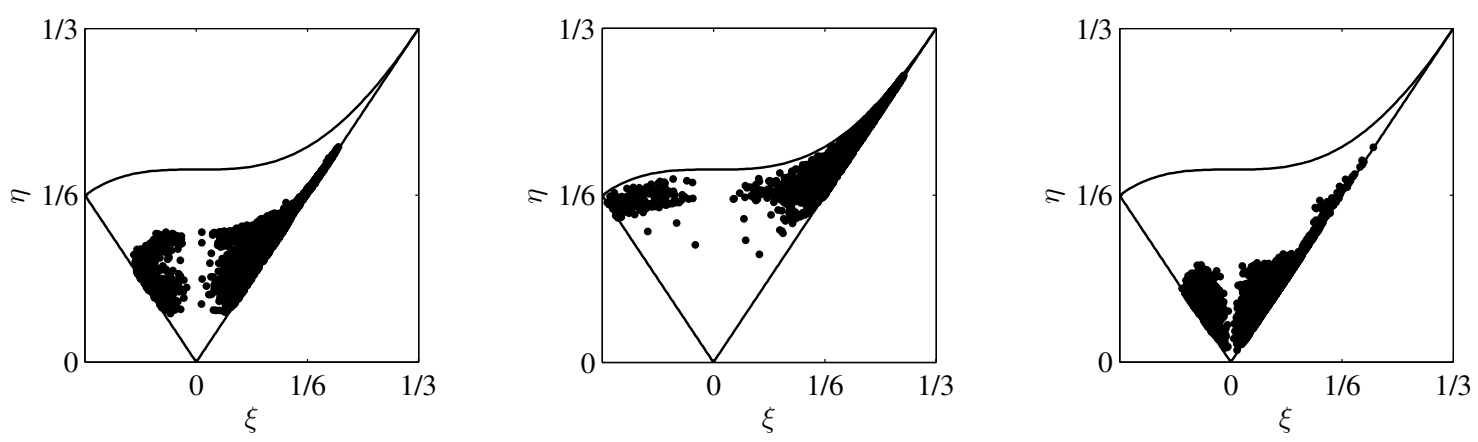

(A) Wind turbine wake, $x / D=0.5$
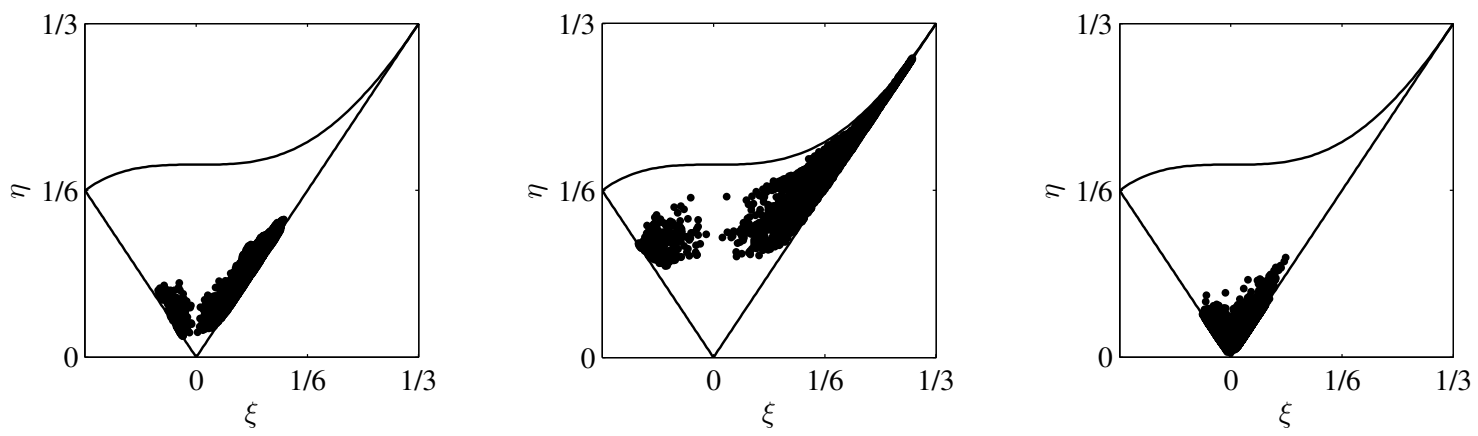

(B) Wind turbine wake, $x / D=6$
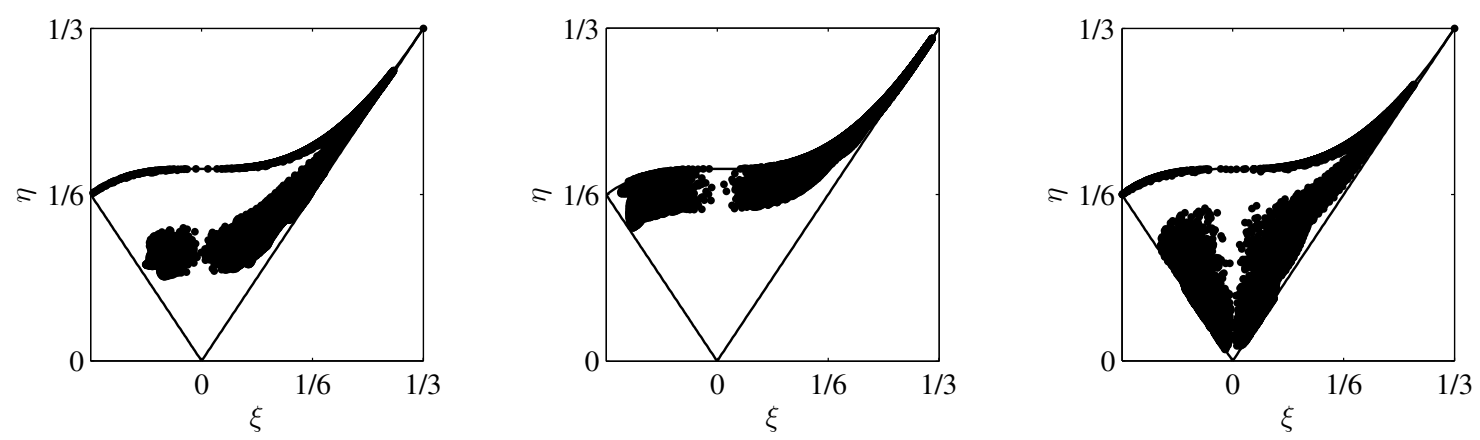

(C) Fully developed channel flow DNS

Figure 4.37: Lumley triangle composed with invariants derived from $\overline{u_{i} u_{j}}$ (left), $\overline{u_{i} u_{j}}$ (center), and $\hat{\overline{u_{i} u_{j}}}$ (right). In each case, the $50 \%$ threshold on $N_{r}$ is used.

respectively.

The center column of the figure plots $\stackrel{\circ}{\eta}$ and $\stackrel{\circ}{\xi}$ for the three data cases. All measurement points exhibit invariants that are greater than in the original data, with the excep- 
tion of data describing one- or two-component turbulence (these points already show the greatest magnitudes of $\eta$ allowed for realizable turbulence). These results confirm that the lowest ranking POD modes correspond with the least isotropic contributions to the turbulence field, as the magnitude of $\eta$ correlates with the anisotropy of the turbulence. Further, the data suggest that low order descriptions of the flow 'flatten' turbulence, moving from fully three dimensional states toward two-component turbulence. Three dimensional turbulence requires three principle stresses for complete description. In contrast, a two-component turbulence field requires only two principle stresses, the orientation of which vary with location. Flattening of the turbulence field is related to the degree of truncation of the POD basis.

The complimentary effect is observed for the isotropic contribution to the flow. Invariants for isotropic contributions $\hat{\eta}$ and $\hat{\xi}$ are compared in the right column of figure 4.37. For representations of the flow using intermediate and high-rank POD modes, the invariants tend toward the isotropic condition where $\hat{\eta}=\hat{\xi}=0$. In the channel flow, there are points that contradict the tendency of invariants to decrease, located in the near-wall region. Areas where $\hat{\eta}$ and $\hat{\xi}$ indicate less isotropic flow coincide with locations where $-\overline{u w}=0$ at the wall. In both of the sampled measurement windows from the wind turbine wake, $\hat{\eta}$ and $\hat{\xi}$ are everywhere smaller in magnitude than $\eta$ and $\xi$. Decreasing the threshold associated with $N_{r}$ exaggerates the anisotropy seen in $\stackrel{\eta}{\eta}$ and $\stackrel{\circ}{\xi}$ while relaxing the distortion seen in $\hat{\eta}$ and $\hat{\xi}$. In using a truncated basis of POD modes for low-order models, a small number of modes greatly reduces the complexity of the resulting model. Extreme reductions of the invariants leads to cases where the turbulence becomes identically one- or two-dimensional. Truncating the basis of POD modes used in low-order descriptions of the flow are shown analytically in appendix A. 
Recalling that the isotropic portion accounts for the turbulence field that is missed by the reduced order representation, the results in figures 4.34 through 4.36 indicate that relatively simple corrections may account for energy missing from $\frac{\circ}{u_{i} u_{j}}$, increasing the accuracy of the low-order description. Several such corrections are explored in the following section.

\subsubsection{Error propagation via basis truncation}

An important consideration in gauging the quality of a low order representation of the turbulence field is the accuracy of each component of the reconstructed stress field. Figure 4.38 shows the root-mean-square (RMS) error between the turbulent stresses derived by statistical means and those from POD reconstructions. Each set indicates that for POD reconstructions with very few modes, RMS error is greatest for the streamwise normal stress. This remains true for all points of truncation in the case of the channel flow. However, in the wind turbine wake data, a truncated modal basis in-

cluding sufficient modes $N_{r}$ exhibits greater RMS error for $\frac{\circ}{\nu v}$ and $\frac{\circ}{w w}$. In all cases, the shear terms of the stress tensor reconstruct with less error than the normal stresses. Because the shear terms contribute to the anisotropy tensor that includes structures favored by low-rank POD modes, they reconstruct faster than normal stresses. As the anisotropic features come out of the POD basis first, the shear terms are wellrepresented with few modes. In the wake data, the spanwise normal stress is associated with large structures captured by the POD. In the wake data, spanwise homogeneity leads to decreased magnitudes of $\overline{w w}$ and low-rank POD modes fail to accurately reconstruct the spanwise normal stress. Interestingly, the RMS error associated with $\frac{\circ}{w w}$ for the channel flow (figure $4.38(\mathrm{C})$ ) is much larger relative the other stresses for 
that data. The reduced magnitude of the RMS error of $\frac{\circ}{u_{i} u_{j}}$ for the channel flow is attributed to the rate at which the flow is recovered via POD. If the present error analysis was performed with data from the channel half-height rather than the inner regions of the boundary layer, more modes would be required to represent the dynamics of the RMS error would be greater.

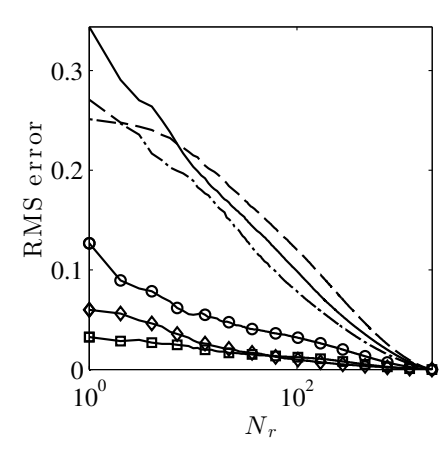

(A) Wind turbine wake, $x / D=(B)$ Wind turbine wake, $x / D=6$ 0.5

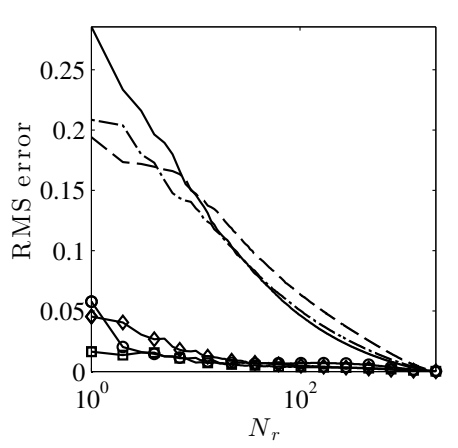

B) Wind turbine wake, $x / D=6$

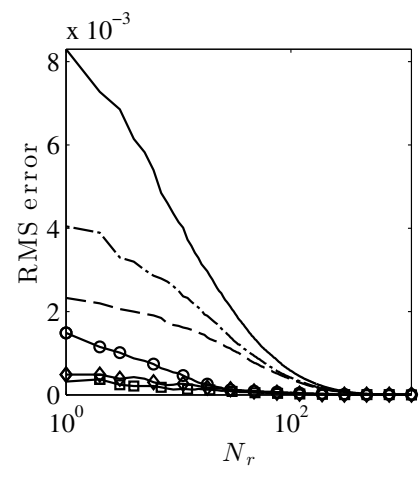

(C) Channel flow DNS

Figure 4.38: RMS error associated with reconstructed Reynolds stress tensor as a function of number of basis modes. In all subfigures, $\overline{u u}$ solid black lines, $\overline{v v}$ dashed lines, $\overline{w w}$ dash-dot lines, $-\overline{u v}$ circles, $-\overline{u w}$ diamonds, $-\overline{v w}$ squares.

A similar gauge of quality for low-order representations compares the RMS error of the invariants of the anisotropy tensor as functions of the number of modes included in the truncated POD basis. Due to the definition of $\eta$ and $\xi$ from the Reynolds stresses, it is expected that the error propagation of low-order representations of the invariants will be similar to that seen for the turbulence stresses. Interestingly, the RMS error of the anisotropy tensor invariants are quite similar from case to case. For all data, the error of $\xi$ is greater than for $\eta$; the RMS error of $\xi$ with $N_{r}=1$ is approximately 25\%. The RMS error of the second invariant at $N_{r}=1$ is less similar between the cases; $\eta$ shows a maximum error of approximately $23 \%$ for $x / D=6$ and a minimum error 
of approximately $17 \%$ for the channel flow. Because the wake data have a different number of modes than the channel flow, the mode numbers have been normalized by the respective total number of modes for each case.

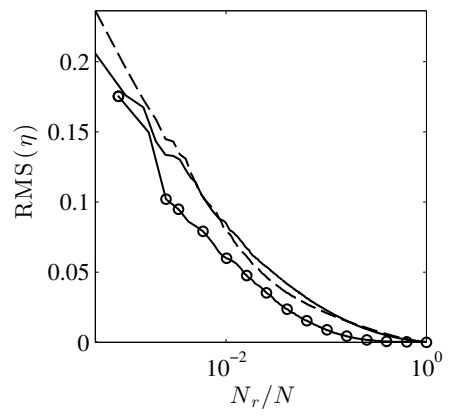

(A) RMS error of $\eta$

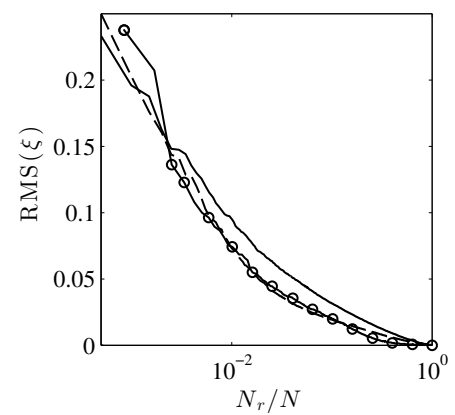

(B) RMS error of $\xi$

Figure 4.39: RMS error of anisotropy tensor invariants as a function of number of basis modes $\left(N_{r}\right)$. Mode numbers are normalized by the total number of modes, $N$. Lines are $x / D=0.5$ (solid black lines), $x / D=6$ (dashed lines), and the channel flow DNS data (circles).

It is from the invariants of the normalized Reynolds stress anisotropy tensor that information is gleaned regarding the quality of the low-order representations of turbulence. The invariants describe the relative balance of elements in the Reynolds stress tensor and describe the state of turbulence in quantifiable terms. The RMS error of the invariants provides a quantitative account of the ability of a low-order representation to match statistics in the turbulence field. A visualization of the quality of loworder representation is provided in figure 4.40, wherein the AIM of the channel flow is composed with varying numbers of modes. Increasing $N_{r}$ leads the invariants to their original values, conforming to the reduction of RMS error discussed above.

A few points of interest arise in the figure regarding the ability of POD descriptions to represent the actual turbulence field. Figure $4.40(\mathrm{~A})$ indicates that using a 


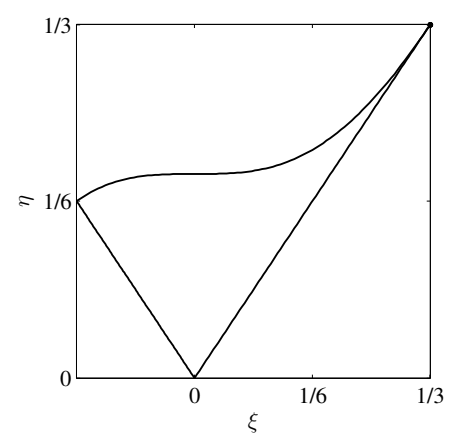

(A) Error: $\eta$ - $17.5 \%, \xi-23.8 \%$

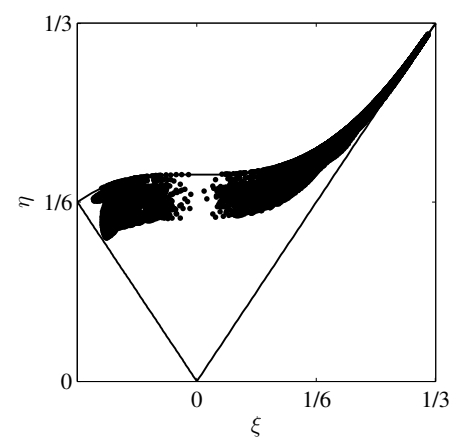

(D) Error: $\eta-7.4 \%, \xi-8.9 \%$

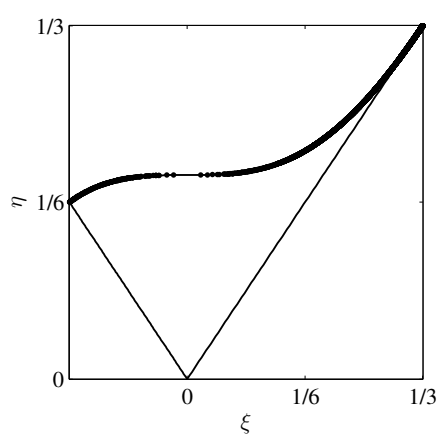

(B) Error: $\eta-15.0 \%, \xi-20.7 \%$

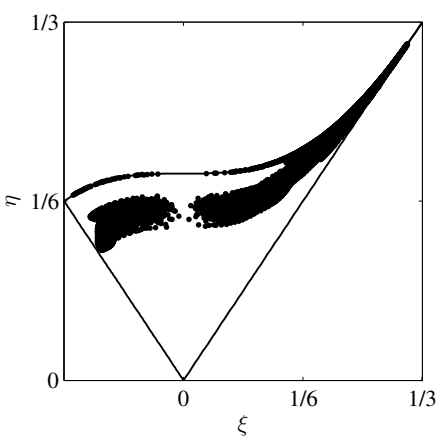

(E) Error: $\eta-5.4 \%, \xi-6.6 \%$

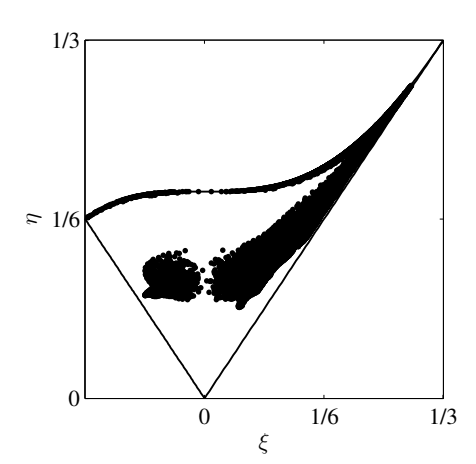

(G) Original data

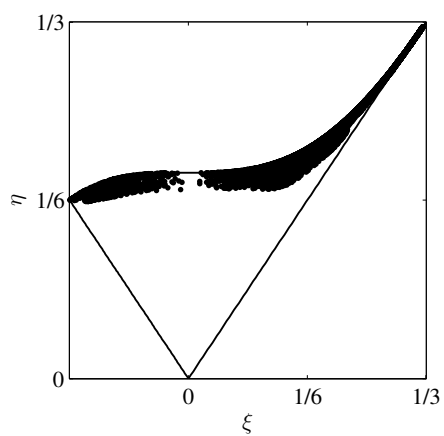

(C) Error: $\eta-9.5 \%, \xi-12.3 \%$

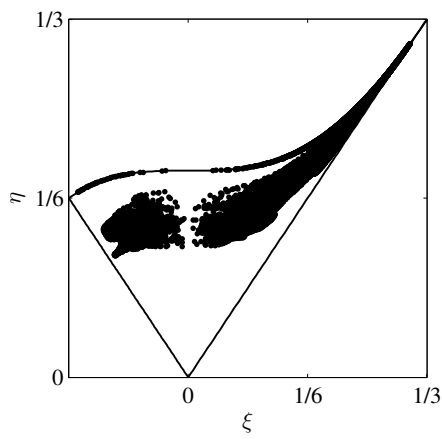

(F) Error: $\eta-3.4 \%, \xi-4.3 \%$

Figure 4.40: Lumley triangle for channel flow represented with increasing number of POD modes. 4.40(A) $N_{r}=1$ (all data fall on a single point where $\eta=\xi=1 / 3$ ), 4.40(B) $N_{r}=2,4.40$ (C) $N_{r}=4,4.40$ (D) $N_{r}=8,4.40$ (E) $N_{r}=16,4.40$ (F) $N_{r}=32$, $4.40(\mathrm{G})$ full data for reference. 
single mode is capable of formulating exclusively one-dimensional turbulence. Similarly, reconstruction with $N_{r}=2$ (Figure 4.40(B)) is capable of reproducing identically two-dimensional turbulence only. It should be noted here that severe basis reductions $\left(N_{r}=1\right.$ and $\left.N_{r}=2\right)$ still require three components of velocity to describe the turbulence field in the domain. The one- or two-dimensional turbulence is a local flattening only; while only one or two principle stresses are needed to describe the local stress field, their orientation changes in the domain, requiring that a global description requires three velocity components. The reduction to one-and two-dimensional turbulence occurs identically for the wake data, although those Lumley triangles are not shown. Analytical demonstrations of the low-order descriptions with $N_{r}=1$ and $N_{r}=2$ are provided in Appendix A.

For $N_{r} \geq 3$, low-order representations describe three-dimensional turbulence field. Figures 4.40(C) through 4.40(F) show the AIM for invariants derived with models using $N_{r}=4,8,16$, and 32 modes, respectively. In each plot, the region of the Lumley triangle spanned by $\stackrel{\circ}{\eta}$ and $\stackrel{\circ}{\zeta}$ approach the span described by $\eta$ and $\xi$, provided in $4.40(\mathrm{G})$ for reference. RMS error for each low-order representation is included in the captions, matching the error propagation in Figure 4.39. In figures 4.40(C) and 4.40(D) the range of invariants is constrained to the upper region of the AIM as a result of the exclusion of the isotropic contribution to the Reynolds stress tensor. Increasing the truncation point to $N_{r}=16$ in figure $4.40(\mathrm{E})$, the invariants demonstrate behavior seen in the full statistics. The one- and two-dimensional behavior seen in the innermost region of the boundary layer is distinct from the three-dimensional turbulence seen in the outer boundary layer. As more modes are included, the low-order description contains more isotropic background turbulence and the invariants move downward, toward their re- 
spective positions in the AIM described by $\eta$ and $\xi$, figure $4.40(\mathrm{G})$. As the POD representation tends toward the state derived statistically, the balance of terms $\frac{0}{u_{i} u_{j}}$ more closely resembles that seen in $\overline{u_{i} u_{j}}$, a tendency reflected in $\stackrel{\circ}{\eta}$ and $\stackrel{\circ}{\xi}$.

\subsubsection{Correction of the reduced order flow description}

A simple form of low-order flow description correction arises from the note that isotropic contribution to the full turbulence fields are both small and fairly homogeneous. This indicates that the energy excluded from the flow using $\frac{\circ}{u_{i} u_{j}}$ may be considered as nearly constant background energy. Recent extensions of the double POD (Hamilton et al., 2016) corrected estimates of the Reynolds stresses by way of a constant coefficients used to push the magnitudes of each component toward the values seen in the full statistics. The basic formulation of such a correction is,

$$
\overline{u_{i} u_{j}}=C_{i j} \bar{\circ} \overline{u_{i} u_{j}}
$$

The correction coefficient $C_{i j}$ is found through a minimization of the root-meansquare error $e r r_{i j}$ between the statistical stress field and the corrected reduced order model,

$$
C_{i j} \ni \min \left[\sqrt{\left\langle\left(\overline{u_{i} u_{j}}-\left.C_{i j} * \frac{\circ}{u_{i} u_{j}}\right|_{N_{r}}\right)^{2}\right\rangle}\right]=\min \left[\operatorname{err}_{i j}\right]
$$

The correction applied to each component of $\frac{\circ}{u_{i} u_{j}}$ is a constant that minimizes the RMS error and effectively matches the magnitudes of the low order descriptions to the statistical values. Correction of this type is attractive in that it is quite simple to derive and apply. However, there remains some error in the corrected stress fields arising 
from heterogeneity in $\hat{\overline{u_{i} u_{j}}}$ that is ignored in the minimization of error. As $C_{i j}$ accounts for the difference between the turbulence field and its low-order approximation, it is necessarily a function of the number of modes used to compose $\frac{\circ}{u_{i} u_{j}}$. Figures 4.41 and 4.42 show $C_{i j}$ and the RMS error between $\overline{u_{i} u_{j}}$ and $C_{i j} \bar{\circ} \overline{u_{i} u_{j}}$, respectively.

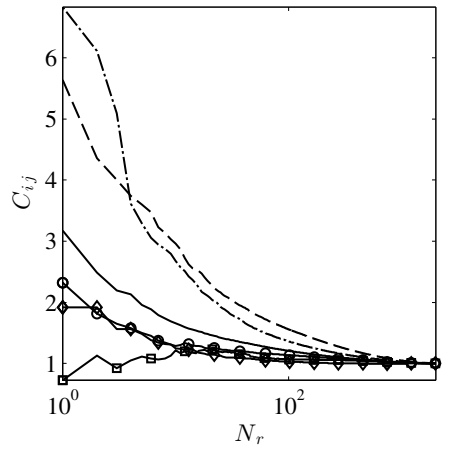

(A) $x / D=0.5$

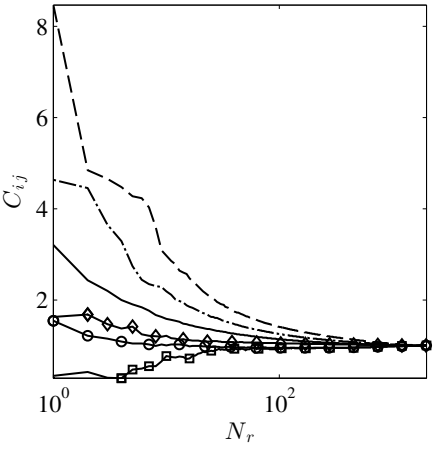

(B) $x / D=6$

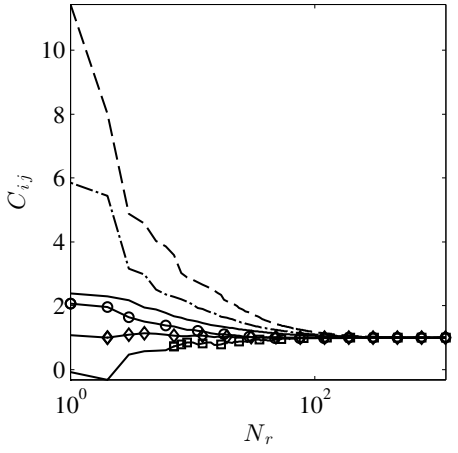

(C) Channel flow

Figure 4.41: Correction coefficient $C_{i j}$ as a function of $N_{r}$. In all subfigures, $\overline{u u}$ solid black lines, $\overline{v v}$ dashed lines, $\overline{w w}$ dash-dot lines, $-\overline{u v}$ circles, $-\overline{u w}$ diamonds, $-\overline{v w}$ squares.

Seen in the figure 4.41 , the corrections associated with the normal stresses are larger in every case than for the shear stresses. This result is expected as the reconstruction of energy by the POD is slower in the normal stresses than in the shear terms. The corrections $C_{2,2}$ and $C_{3,3}$ applied to the wall-normal and spanwise normal stresses are greatest in each each case and for all POD truncations. $C_{i j}$ falls off quickly for each case, where $C_{i j}<2$ for all components beyond $N_{r} \approx 100$. An unanticipated result comes in the correction for the in-plane shear stress $-\frac{0}{v w}$. This correction, unlike the others, is less than unity, which implies that the low-order description over estimates the energy using a truncated basis. In each of the data sets explored here, the in-plane shear stress has the least energy of the Reynolds stress tensor. All other correction terms are strictly greater than unity, signifying that energy is excluded in the 
POD reconstruction.

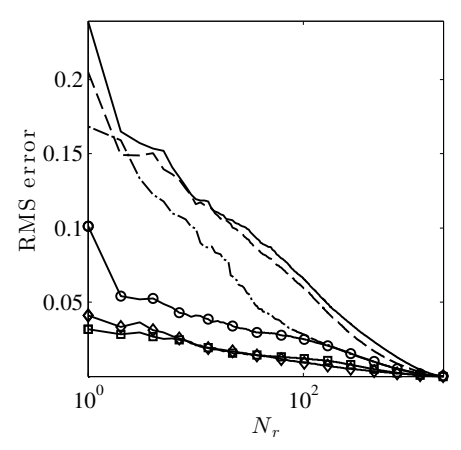

(A) $x / D=0.5$

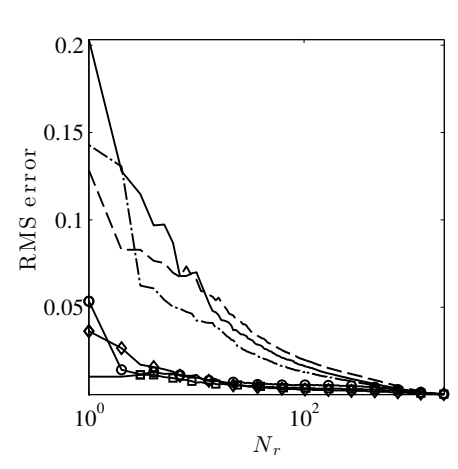

(B) $x / D=6$

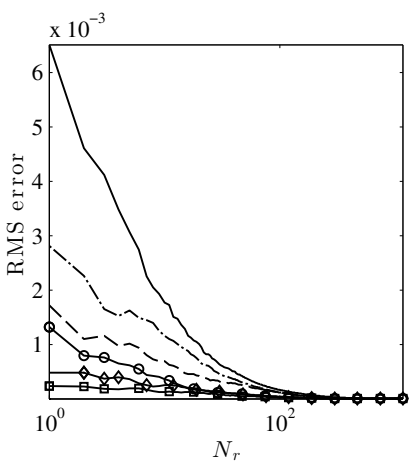

(C) Channel flow

Figure 4.42: RMS error between $\overline{u_{i} u_{j}}$ and $C_{i j} \frac{\circ}{u_{i} u_{j}}$. In all subfigures, $\overline{u u}$ solid black lines, $\overline{v v}$ dashed lines, $\overline{w w}$ dash-dot lines, $-\overline{u v}$ circles, $-\overline{u w}$ diamonds, $-\overline{v w}$ squares.

Correction with $C_{i j}$ leads to a significant reduction in RMS error for all stresses and all cases. Figure 4.42 details the error of the corrected POD reconstructions according to the definition in equation (4.11). The maximum error for each case is associated with $C_{1,1} \frac{\circ}{u u}$, similar to the error seen in the uncorrected low-order representations. The error with shear stresses is maximum for the $-C_{1,2} \frac{\circ}{u v}$ from the data located at $x / D=0.5$ but falls of quickly to be less than $5 \%$ everywhere. Comparing the RMS error of $\frac{\circ}{u_{i} u_{j}}$ to the RMS error of $C_{i j} \frac{\circ}{u_{i} u_{j}}$ indicates that truncation error is reduced by $30 \%$ for the wake data and about $25 \%$ for the channel flow.

Numerical values for correction coefficient tensors and associated RMS error are provided below. The particular values provided correspond to the $50 \%$ threshold of $\lambda^{(n)}$ seen above for the near wake, far wake, and channel flow in figures 4.34 through 4.36 . 


$$
\begin{aligned}
& C_{i j}=\left[\begin{array}{rrr}
1.51 & 1.27 & 1.16 \\
& 2.47 & 1.21 \\
& & 2.17
\end{array}\right] \quad \operatorname{err}_{i j}(\%)=\left[\begin{array}{lll}
10.9 & 3.64 & 1.69 \\
& 10.7 & 1.70 \\
& & 7.89
\end{array}\right] \\
& C_{i j}=\left[\begin{array}{rrr}
1.55 & 1.02 & 1.15 \\
& 2.78 & 0.74 \\
& & 2.01
\end{array}\right] \quad \operatorname{err}_{i j}(\%)=\left[\begin{array}{lll}
5.23 & 0.80 & 0.78 \\
& 5.70 & 0.62 \\
& & 4.09
\end{array}\right] \\
& C_{i j}=\left[\begin{array}{ccc}
1.56 & 1.24 & 1.04 \\
& 2.88 & 0.85 \\
& & 2.12
\end{array}\right] \quad \operatorname{err}_{i j}(\%)=\left[\begin{array}{lll}
0.20 & 0.042 & 0.023 \\
& 0.073 & 0.014 \\
& & 0.14
\end{array}\right] \text {. }
\end{aligned}
$$

The correction tensors in equations (4.12), (4.13), and (4.14) indicate a similarity in behavior of the POD reconstructions from case to case. Consistently, the normal stresses require the greatest correction as they accumulate energy from a greater number of POD modes. In the cases of the wake at $x / D=6$ and the channel flow, the correction associated with $-\frac{\circ}{v w}$ is less than unity, $C_{2,3}<1$ which implies that the low-order representation of that stress is over estimated and must leak energy out in intermediate and higher modes.

Error associated with correction of this form is within 5\% except in the near wake of the wind turbine. There, the maximum error of approximately $11 \%$ arises from the difficulty of reproducing the complex features from the passage of the rotor blades. The other data sets also display the greatest error associated with $\overline{u u}$, although the associated error is less than that shown for $x / D=0.5$. Because the shear terms result from 
anisotropic turbulence and reconstruct with fewer modes, they are also represented most accurately with the minimization approach to correction.

Correction of this form is nonlinear and has effects in the overall balance of the modeled Reynolds stresses that in turn alter the behavior of the anisotropy tensor invariants. Each component of the correction tensor is defined to minimize the RMS error of $\frac{\circ}{u_{i} u_{j}}$ with the respective component of $\overline{u_{i} u_{j}}$. Energy is distributed to each component of the stress tensor at a different rate and the corrections attempt to account for energy excluded from the truncated basis. A constant correction coefficient imperfectly assumes that energy is excluded homogeneously in the domain, leading to the RMS errors seen in equations (4.12) through (4.14) above. Despite any remaining deviation from the original statistical field, it is encouraging to note that such model correction can account for significant improvements in the behavior of the low-order invariants $\stackrel{\circ}{\eta}$ and $\stackrel{\circ}{\xi}$. Figure 4.43 demonstrates the anisotropy tensor invariants derived from the low-order description of the turbulence field corrected with $C_{i j}$.

Most notably, the AIM with a $N_{r}=1$ and $N_{r}=2$ (figures 4.43(A) and 4.43(B)) are no longer constrained to one- and two-dimensional turbulence. Under correction, severe model reduction is capable of reproducing three dimensional turbulence. As before, increasing the number of modes used in the low-order description leads to invariants that more closely match those of the original stress field. In each of the corrected AIMs, the minimum values of $\stackrel{\circ}{\eta}$ are nearly identical to those of $\eta$ (Figure 4.43), indicating that for at least some of the domain, the correct degree of isotropy is generated in the corrected turbulence fields. The two-dimensional behavior seen in the inner boundary layer is unaffected by the correction and reconstructs accurately with a mode basis of any size. As it does for the Reynolds stress tensor, $C_{i j}$ reduces the RMS error between 
the anisotropy tensor invariants and their respective low-order approximations.

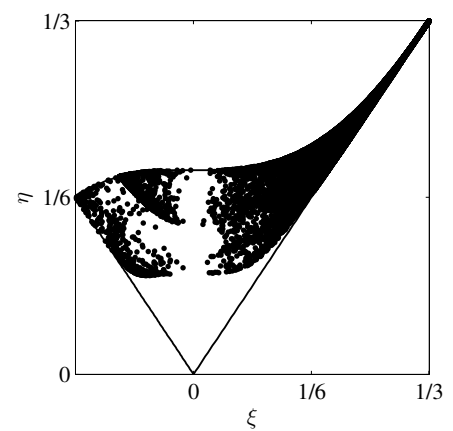

(A) Error: $\eta-12.2 \%, \xi-14.6 \%$

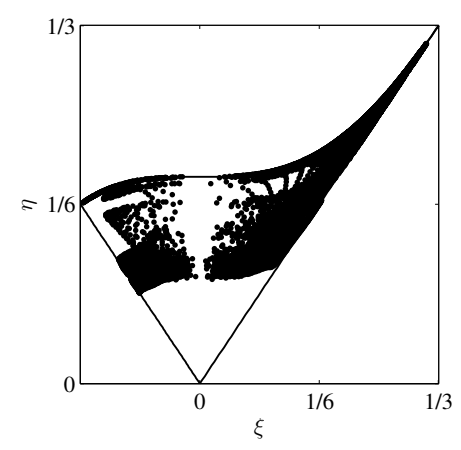

(D) Error: $\eta-3.8 \%, \xi-7.7 \%$

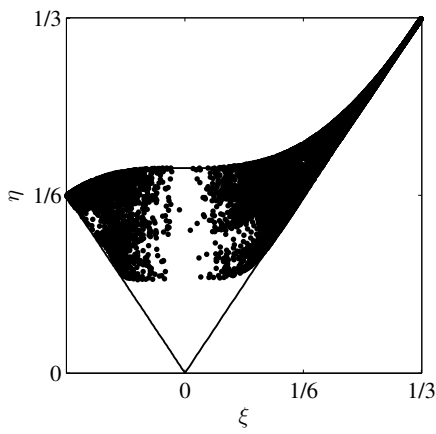

(B) Error: $\eta-8.5 \%, \xi-15.3 \%$

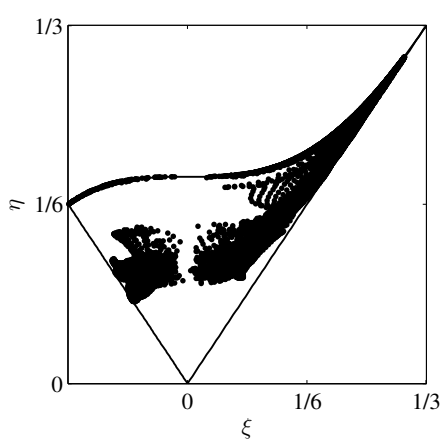

(E) Error: $\eta-1.8 \%, \xi-6.0 \%$

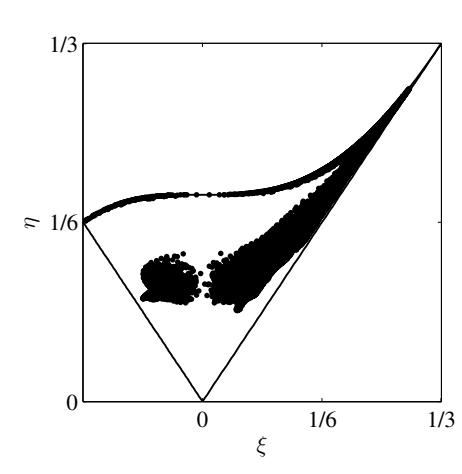

(G) Original data

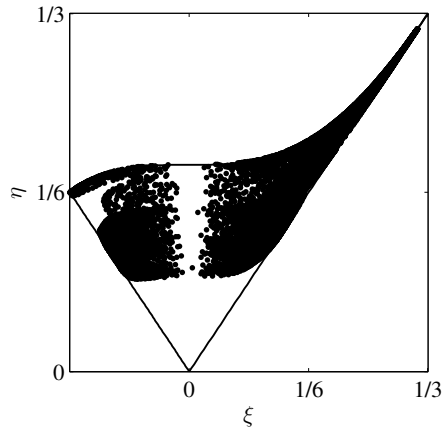

(C) Error: $\eta-5.6 \%, \xi-9.4 \%$

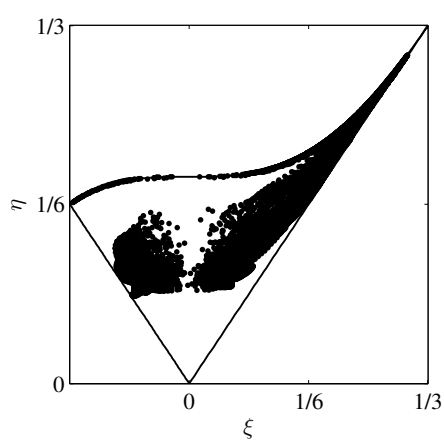

(F) Error: $\eta-1.2 \%, \xi-1.2 \%$

Figure 4.43: Lumley triangle for channel flow represented with increasing number of POD modes and correction factor $C_{i j}$. 4.43(A) $N_{r}=1,4.43(\mathrm{~B}) N_{r}=2,4.43(\mathrm{C})$ $N_{r}=4,4.43(\mathrm{D}) N_{r}=8,4.43(\mathrm{E}) N_{r}=16,4.43(\mathrm{~F}) N_{r}=32,4.43(\mathrm{G})$ full data for reference. 
Although behavior of the low-order invariants seen in figure 4.43 is much closer to $\eta$ and $\xi$ than the uncorrected versions, is clear that error remains in the low-order description. The RMS error between the corrected low-order invariants and their respective originals values is shown in figure 4.44. As done earlier, the mode number $N_{r}$ has been normalized by the respective total number of modes for each case, such that they may be plotted together. Application of $C_{i j}$ to the low-oder description reduces the RMS error of the resulting anisotropy invariants by approximately $50 \%$ for both $\eta$ and $\xi$. Interestingly, the RMS error of $\xi$ for the channel flow is seen to increase from $N_{r}=1$ to $N_{r}=2$.

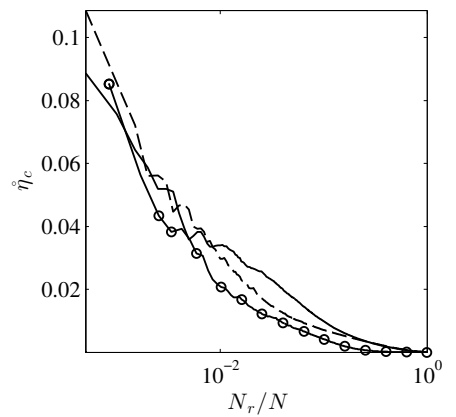

(A) $x / D=0.5$

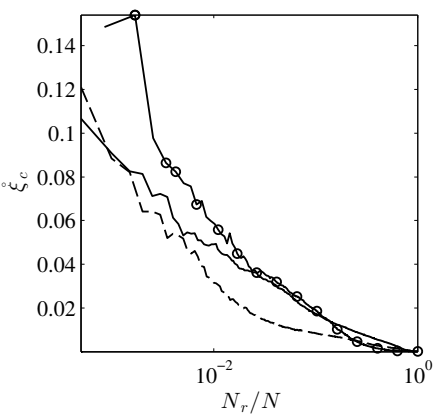

(B) $x / D=6$

Figure 4.44: RMS error between anisotropy invariants derived from corrected stress tensor and statistical values.

Another form of model correction is approached analytically based on the division of the POD basis. As above, the POD basis is divided into parts corresponding to the anisotropic and the isotropic contributions to the turbulence field.

$$
\sum_{n=1}^{N} \lambda^{(n)} \phi_{i}^{(n)} \phi_{j}^{(n)}=\sum_{n=1}^{N_{r}} \lambda^{(n)} \phi_{i}^{(n)} \phi_{j}^{(n)}+\sum_{n=N_{r}}^{N} \lambda^{(n)} \phi_{i}^{(n)} \phi_{j}^{(n)}
$$

where $N_{r}$ is the truncation point and the energy expressed in the isotropic contribution 
of the turbulence field. Recalling that the stress tensor may be expressed as superposition of the products of POD modes and their respective eigenvalues, equation (4.15) may be rewritten as,

$$
\overline{u_{i} u_{j}}=\bar{\circ} \overline{u_{i} u_{j}}+\overline{u_{i} u_{j}}
$$

which assimilates the definitions presented in (4.7) through (4.9). The previous correction $C_{i j}$ sought to minimize error associated with basis reduction with a constant. Correction with a constant springs from the assumption that energy in $\hat{u_{i} u_{j}}$ is homogeneously distributed. While the correction leads to a global reduction in error of the low-order representation, there remain artifacts arising from the basis truncation. The coefficient tensor $C_{i j}$ may also lead to distortion of the anisotropic quality of the loworder description through imperfect correction.

Given that remaining error results from inhomogeneous distribution of the isotropic contribution to the turbulence field, a spatially explicit correction may be necessary to fully correct the POD approximation. Combining equation (4.22) and equation (4.16) provides an analytical expression for a correction factor as a function of the isotropic and anisotropic contributions to the turbulent stress field,

$$
D_{i j}=\left(1+\frac{\hat{u_{i} u_{j}}}{\overline{\frac{o}{u_{i} u_{j}}}}\right) .
$$

The correction factor now accounts for all of the energy excluded in model basis truncation but is a function of the spatial coordinates $\vec{x}$. Another consideration in $D_{i j}$ is that the correction becomes singular any time the low-order description of any component of the stress tensor is orders of magnitude smaller than the isotropic con- 
tribution. Energy expressed by either $\frac{\circ}{u_{i} u_{j}}$ or $\hat{u_{i} u_{j}}$ changes according to the point of truncation $N_{r}$. Accordingly, we expect that $D_{i j}=D_{i j}\left(\boldsymbol{x}, N_{r}\right)$.

Figure 4.45 shows $D_{i j}$ associated with Reynolds stresses including fluctuations of the streamwise velocity, $\overline{u u},-\overline{u v}$, and $-\overline{u w}$. The correction factor is calculated according to equation (4.17) and is shown in logarithmic scale because the correction becomes singular whenever the anisotropic stress is zero. As for the low-order representations of stresses shown in figures 4.34 through 4.36 , the truncation point used in each case in figure 4.45 corresponds with the $50 \%$ threshold of $\lambda^{(n)}$.

At $x / D=0.5$, the correction $D_{1,1}<1$ everywhere except following the nacelle and the mast of the model wind turbine. Correction greater than unity indicates that the contribution to the stress field from intermediate- and high-rank POD modes dominates the contribution from low-rank modes. The correction needed for the shear terms is on the order of unity, as expected. From the low-order representations of the turbulence field (figures 4.34 through 4.36), the shear terms of $\hat{u_{i} u_{j}}$ are nearly null across most of the measurement domain; thus the ratio of isotropic to anisotropic shear stresses is nearly null and $D_{i j} \approx 1$ from equation (4.17).

Similar behavior is seen in the contours of $D_{i j}$ from $x / D=6$. There the correction associated with $\stackrel{\circ}{u u}$ again shows greater magnitude following the mast and nacelle of the model turbine. The correction $D_{1,1}$ is generally less than for $x / D=0.5$ following the more complete representation of the turbulence with $\frac{\circ}{u u}$ at that location. The shear terms demonstrate more frequent extreme values of $D_{1,2}$ and $D_{1,3}$ than in the near wake, which arise from the decreased magnitudes of the anisotropic turbulence compared to the isotropic contributions at $x / D=6$.

The turbulence field in the case of the channel flow is more uniform than that of the 

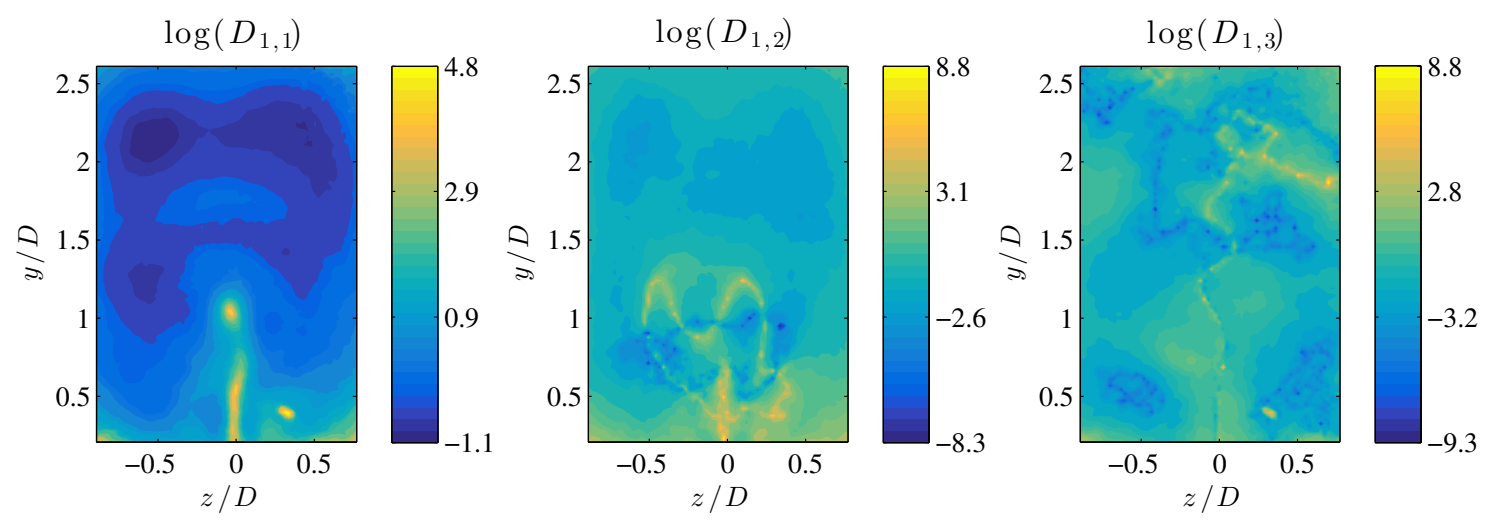

(A) $x / D=0.5$
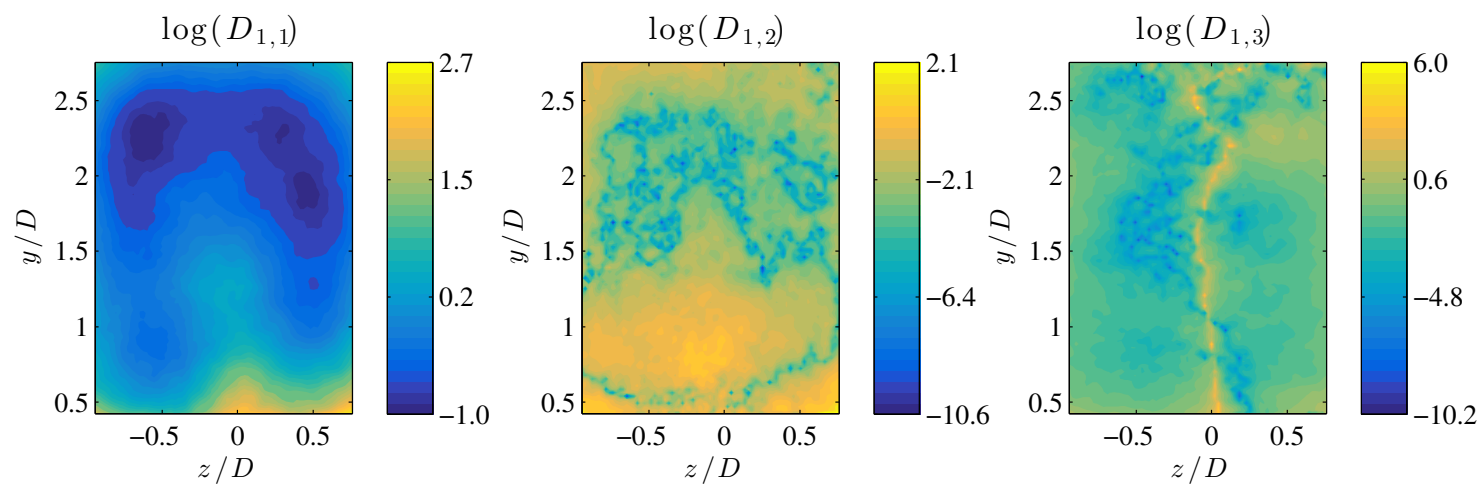

(B) $x / D=6$
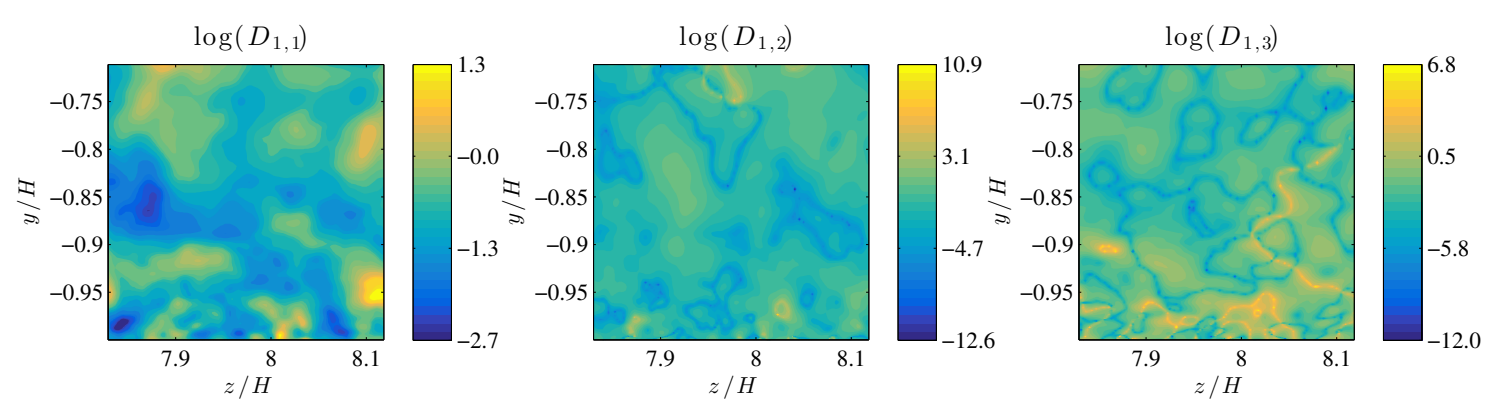

(C) Channel flow

Figure 4.45: Correction to selected stresses using the ratio of isotropic to anisotropic contributions. Due to extreme values, the logarithm of the correction tensor $\log \left(D_{i j}\right)$ is shown. 
wind turbine wake, which leads to more uniform corrections shown in figure 4.45(C). Corresponding to the contours of $\hat{u} u, D_{1,1}$ is less than unity for most of the domain. The exception occurs in the near wall region, where $\stackrel{\circ}{u u}$ is least accurate and more energy is left in the isotropic range of turbulence. The shear terms of $D_{i j}$ do not shown any distinctive features seen in the contours of $\overline{u_{i} u_{j}}$ or $\overline{u_{i} u_{j}}$. The error associated with the low-order descriptions $-\frac{\circ}{u v}$ and $-\frac{\circ}{u w}$ are less than $1 \%$ before correction, implying that little information is excluded from the low-order approximation. In $D_{1,3}$ for all data sets there is a vertical yellow line where $D_{1,3} \gg 0$. At these locations the algebraic sign of $-\frac{\circ}{u w}$ changes with respect to $z / D$. Although the value of the correction is quite large there, it is strictly positive because the algebraic sign of $-\hat{u w}$ is the same as $-\frac{\circ}{u w}$. Points where $-\frac{\circ}{u w} \approx 0$ but the sign is opposite that of $-\hat{u w}$ correspond to the extreme negative values in the domain.

Correction of the POD reconstruction with equation (4.17) results in perfect representation of the statistical stress fields. The definition of the correction leads the RMS error of $D_{i j} \frac{\circ}{u_{i} u_{j}}$ to be identically zero. However, the reduction of error comes at a cost. Namely, the correction is no longer a simple constant coefficient tuning the turbulence field to match the span of the original statistics. Rather, each component is a explicit over the domain of the data, $D_{i j}=D_{i j}(\boldsymbol{x})$. A further drawback is that in defining the correction, the full set of POD modes is required, rather than a truncated subset. The modal information is used directly in $\frac{\circ}{u_{i} u_{j}}$, but the remaining modes are necessary for the correction. Following the exact representation of the stress field by $D_{i j} \frac{\circ}{u_{i} u_{j}}$, the anisotropic state is also reconstructed without residual error. Figure 4.46 confirms that the anisotropy tensor invariants arising from correction by $D_{i j}$ match $\eta$ and $\xi$ exactly. 


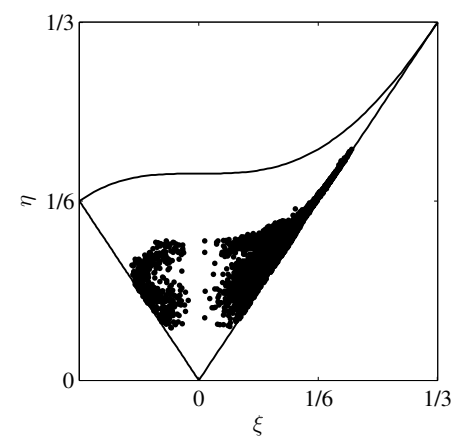

(A) $x / D=0.5$

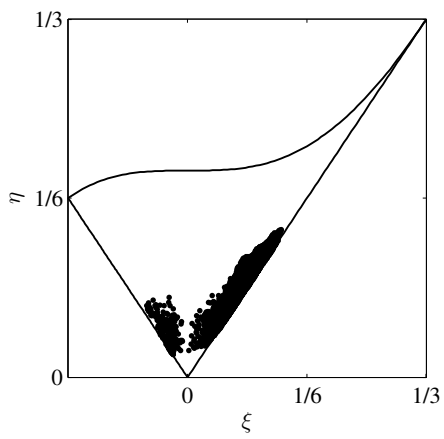

(B) $x / D=6$

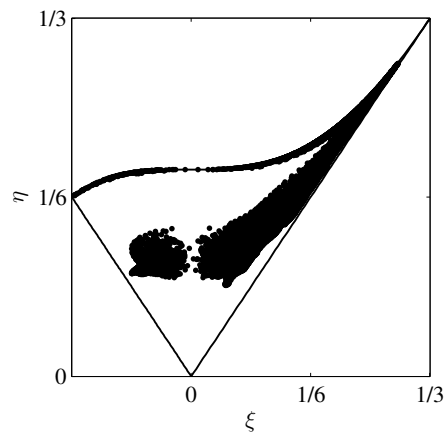

(C) Channel flow

Figure 4.46: Lumley triangles with anisotropy invariants derived from decomposition of $D_{i j} \overline{u_{i} u_{j}}$.

\subsubsection{Conclusions}

The proper orthogonal decomposition is a widely used analytical tool in the study of turbulent fluid flows. Large scales, expressing much of the turbulence kinetic energy, are accounted for by the first POD modes, and often describe the most important features of the flow. Because the POD is able to efficiently describe the flow, POD modes are used in reduced-order models as in Galerkin projection and artificial neural-networks.

Analysis of the invariants of the normalized Reynolds stress anisotropy tensor is a well-developed theoretical platform for characterizing the state of a turbulent flow. The second and third invariants are typically mapped against one another in the Lumley triangle, whose boundaries correspond with special states of turbulence used as limiting cases and boundary conditions. Mapping the invariants in the Lumley triangle provides insight to the balance of the Reynolds stress tensor and provides clues as to the nature of the flow.

The above work samples data from substantially different turbulent flow fields in an 
exploration of the anisotropic quality of low-order descriptions derived through truncated POD bases. Experimental data from a model-scale wind turbine wake is sampled very close to the model device, where the flow is very intermittent and dominated by highly anisotropic turbulence, as well as the far wake, where the turbulence field is well-mixed and relatively isotropic. These data are contrasted with a boundary layer arising from the DNS of a fully developed channel flow. The innermost regions of the boundary layer are resolved clearly in the data and have anisotropic properties conforming to the boundary conditions imposed on the simulation.

Application of the POD to the data sets reveals the expected accumulation of energy expressed by the cumulative summation of POD eigenvalues. Low-order descriptions are made truncating the basis of POD modes according to a $50 \%$ energy threshold, considered common practice in the use of POD. Although this threshold is arbitrary in terms of the error of the low-order description of the turbulence field, the $50 \%$ threshold is used frequently, presumably due to the comfortable equality of turbulence kinetic energy included and excluded of from the resulting approximation of $\overline{u_{i} u_{j}}$. Mapping the propagation of RMS error between the low-order description and the statistically derived turbulence fields demonstrates that although a fair portion of the energy is excluded, the stress field represented with less than 15\% RMS error. Composing the normalized Reynolds stress anisotropy tensor with the low-order description leads to new insights regarding the quality of the POD reconstructions. Error for the anisotropy tensor invariants are greater than the components of the Reynolds stress tensor, arising from the allowable ranges of the invariants for realizable turbulence. Lumley triangles composed with the low-rank POD modes exhibit higher values of $\eta$ and $\xi$ than those derived from the full statistics due to the exclusion of any isotropic turbulence from 
the POD. Contrarily, the intermediate- and high-rank POD modes contribute much more to small-scale and homogeneous turbulence, and result in smaller magnitudes of $\eta$ and $\xi$, confirming that they express mainly isotropic turbulence structures. There is a non-linear relationship between the Reynolds stress tensor and the anisotropy tensor invariants. Thus, there is no intuitive combination of invariants of $\stackrel{\circ}{b j}_{i j}$ and $\hat{b}_{i j}$ that recovers anisotropy tensor invariants conforming to the original data.

A tensor of constant coefficients was defined to correct the magnitudes of each component of the low-order Reynolds stress tensor, minimizing the remaining RMS error. Correction of this form assumes that the energy excluded from the POD approximation is homogeneously distributed isotropic turbulence kinetic energy. Residual RMS error between the corrected POD approximation and the original statistics is approximately $25 \%$ lower than the uncorrected low-order description for the channel flow and approximately $30 \%$ lower for the wind turbine wake. The anisotropy tensor invariants show even greater reduction in residual error, nearly $50 \%$ of the uncorrected version. Most notably, correction with a tensor of constant coefficients effectively rebalances the magnitudes of the Reynolds stresses such that the invariants of the anisotropy tensor are more closely resemble realistic three-dimensional turbulence. In the cases of severe basis truncation, the POD descriptions are able to account for only one- or two-dimensional turbulence. Correction with constant coefficients enables the representation of three-dimensional turbulence for severe basis truncations.

Isotropic contributions to the turbulence field are more accurately accounted for by a second proposed form of correction. A spatially explicit ratio of the isotropic stresses to the anisotropic stresses accounts for variations in the turbulence excluded 
from the truncated POD basis within the measurement domain. When combined with the low-order description derived from a truncated POD basis, the ratio type correction accounts for the excluded energy exactly. Anisotropy tensor invariants are similarly reproduced without residual RMS error. Beyond the initial definition of the correction tensor, one may use a truncated basis for the purposes of reduced-order modeling. Correction of this form is attractive in its ability to fully represent the turbulence field but requires the full POD mode basis in order to define the correction. Additionally, this form of correction is more complex to apply in practice as it varies over the measurement domain.

Through anisotropy tensor invariant analysis, the specific nature of the inaccuracy of low-order models arising from truncated POD basis is made evident. Specifically, it is shown that low-order models underestimate the magnitudes of the Reynolds stresses by excluding energy from the modal basis and simultaneously exaggerate the anisotropy of the flow. The proposed correction methods account for both the exclusion of energy and the distortion of the anisotropy quality of the flow. Similarity apparent in the error propagation of POD models as well as correction tensor suggests that generalized corrections may be made for specific flow types or arrangements. Corrections explored above also make severe basis truncations accessible for modeling of realistic three-dimensional turbulence. 


\subsection{Dynamical systems of a fully developed turbulent channel flow}

Ensemble-averaged mean velocities are shown in Figure 4.47. Sampled data spans half of the total channel width. Full resolution of the channel to this depth is represented by more than 256 points; significant down-sampling has occurred in the presented snapshots and is evident in the contours of the volume shown for mean velocities. Downsampling in this way limits the range of dynamics present in the dataset. However, the aim of the analysis below is to develop a dynamical system capable of accounting for the majority of the dynamics of the input, rather than a complete description of the channel flow.

The mean flow field in the channel shows a streamwise velocity two orders of magnitude of larger than the wall-normal and spanwise components. Reflecting the profile in Figure 3.4, the high-shear region is confined to near the channel wall. The magnitude of $U$ rapidly increases below $y / H \leq 0.8$ and changes little toward the center of the channel. Spanwise variations is seen in Figure 4.63(A), reflecting the down-sampling of the dataset. It is expected that in the full DNS, the mean wall-normal and spanwise velocities approach zero. Although the magnitudes of $V$ and $W$ are small compared to $U$, there are large features visible in Figures 4.47(B) and 4.47(C) resulting from incomplete statistical convergence of the selected data. These features are excluded from the kernel of the POD and, therefore the resulting LODS.

Turbulence in the sampled data is the field of interest in the development of the

Results in the current section as well as the Galerkin projection found in Appendix B have been submitted for publication and are currently undergoing review, Hamilton, N., M. Tutukn, and R. B. Cal. "Low-order dynamical systems modeling of fully-developed turbulent channel flow." Physica D (under review). 


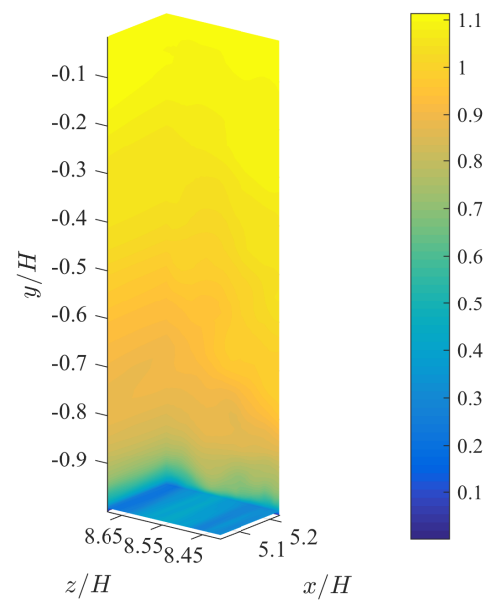

(A) $U$

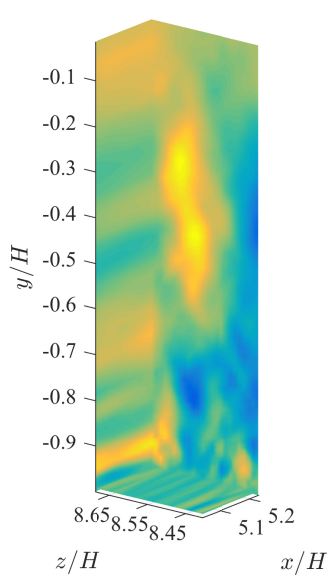

(B) $V$
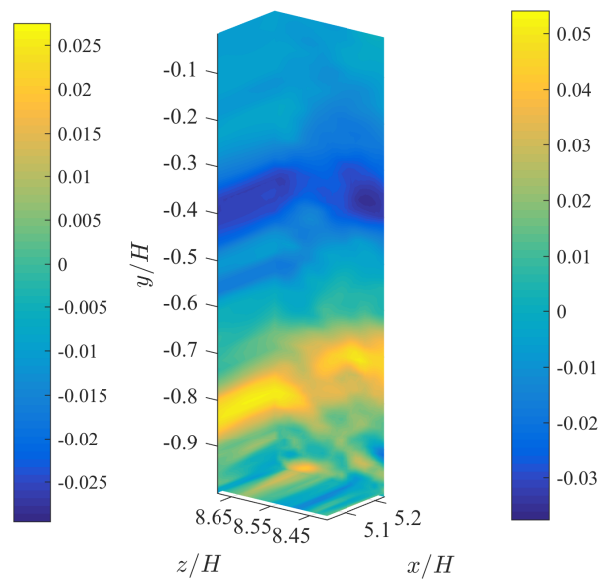

(C) $W$

Figure 4.47: Streamwise, wall-normal, and spanwise mean velocities in channel flow scaled by the bulk velocity.

following dynamical system. Figure 4.48 shows the turbulence kinetic energy $(k=$ $1 / 2 \overline{u_{i} u_{i}}$ ) and two turbulent shear stresses, ensemble averaged in time only. Stress fields in the figure are used as the basis for comparison for the POD representations of the flow field and LODS below. Streaks are evident near the wall, shown in both $k$ and $\overline{u v}$, as well as a larger structure in the lower-right corner of the sampled volume. Larger structures of lower magnitude are seen toward the center of the channel. Systems that exhibit a large range of dynamically significant structures are notoriously difficult to capture with POD based modeling approaches. Wall-normal and spanwise normal stresses are smaller in magnitude than $\overline{u u}$ but show similar size and distribution of turbulence structures. Streaks near the wall in seen in $k$ (Figure 4.48(A)) arise from the contributions of the streamwise and spanwise normal stresses but are absent in $\overline{v v}$.

The streamwise/wall-normal Reynolds shear stress $\overline{u v}$ (Figure 4.65(A)) is the only off-diagonal term from the turbulence stress tensor expected to have a non-null profile 
in statistics from the full DNS. The figure shows several regions of large magnitude below $y / H \leq-0.8$; away from the wall $\overline{u v}$ is comparatively small. It is expected that $-\overline{u w}$ and $-\overline{v w}$ tend toward zero with complete statistical convergence; because the sample analyzed here spans a relatively small space and time interval of the DNS, stresses including fluctuations in the spanwise direction exhibit features on the same order as $\overline{u v}$, demonstrated in Figure 4.65(C). Deviation of $\overline{v w}$ from the fully converged, null statistical profile reflects the turbulence in the sampled volume that occurs within the down-sampled time range. Turbulence statistics from the sampled data shown in Figure 4.48 significantly deviate from the fully converged statistics of the DNS by design. It is of interest in the following analysis to determine whether the proposed dynamical systems can predict turbulence statistics that are more converged than their respective down-sample values.

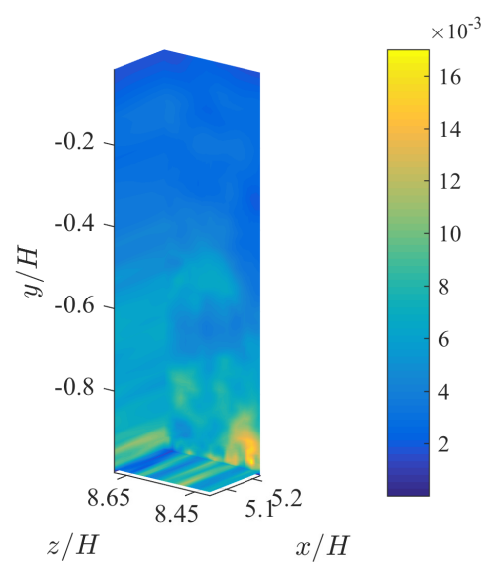

(A) $k$

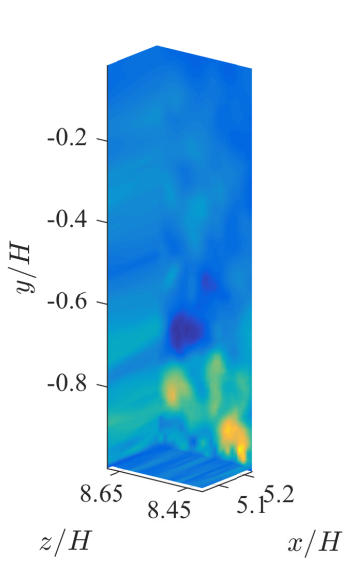

(B) $\overline{u v}$
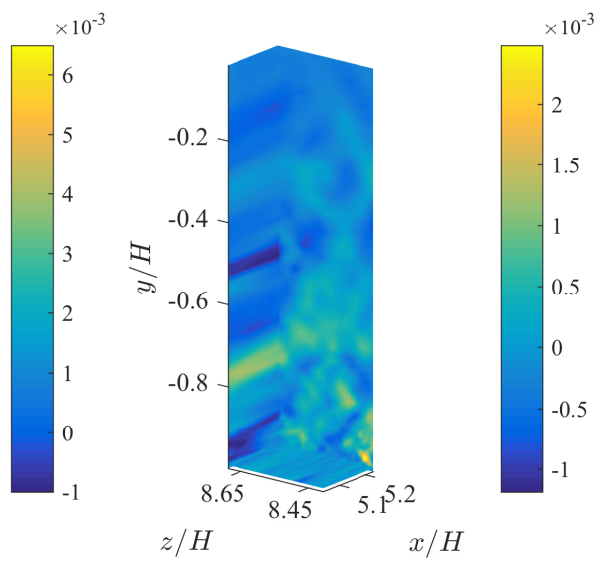

(C) $\overline{v w}$

Figure 4.48: Turbulence kinetic energy and selected Reynolds shear stresses for sampled channel flow volume. 


\subsubsection{POD}

The POD is applied to the sample data where each snapshot is taken as a 3D field of fluctuating velocity. Eigenvalue spectra shown in Figure 4.49 indicate the decay of energy per mode and show several distinct regions of behavior. The low-order modes by definition represent the largest portion of the integrated turbulence kinetic energy in the kernel. The distribution of energy is shown normalized by the summation of the eigenvalues in Figure 4.66(A). The relatively flat portion of the spectrum indicates a relatively large number of energy-containing modes. Beyond $n \sim 10^{2}$, the spectrum falls off quickly indicating a rapid decay in the energy expressed by high-order modes. The cumulative summation of eigenvalues shown in Figure 4.66(B) shows thresholds of the integrated kinetic energy typically used as points of basis truncation.

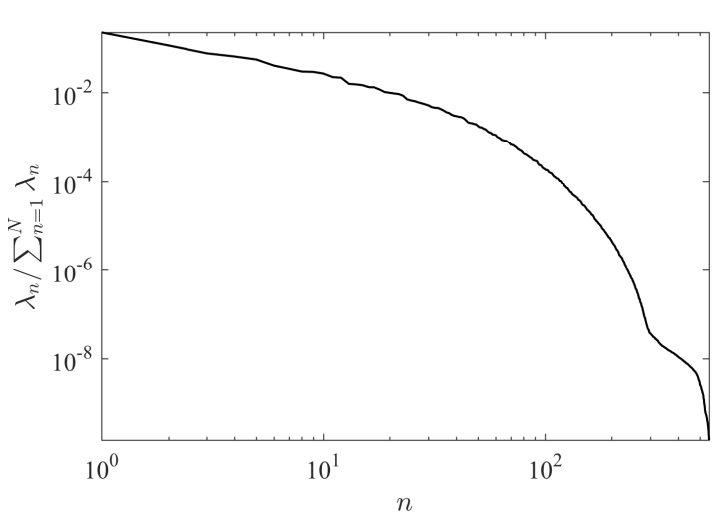

(A)

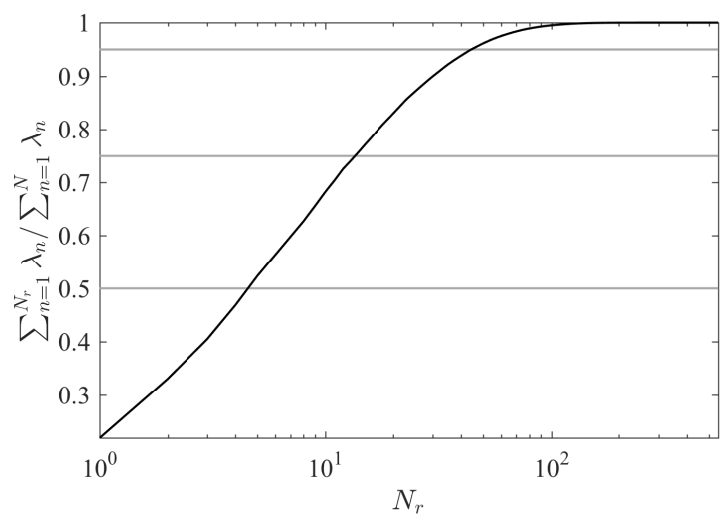

(B)

Figure 4.49: Normalized POD eigenvalues (a) and their cumulative summation (b) along with energy thresholds. 5-modes are needed to reach the $50 \%$ energy threshold.

In using a volume of data, the ability of the POD to quickly account for the full character of the flow is diminished and the resulting eigenvalue spectrum is flattened accordingly. The transverse and streamwise directions are characterized by periodic 
boundary conditions, and introduce homogeneity to the kernel of the POD, which causes modes to tend toward Fourier-type behavior, thus retarding the convergence of the basis. Time resolution of the velocity snapshots is equal to the maximum resolution of the JHUCF data stored from the simulation; every 0.065 dimensionless time units. At this resolution, velocity snapshots are not statistically independent, and correlation is expected between successive fields. The resulting POD coefficients are not considered 'random' as they are in other applications of the POD, but rather are timeseries.

According to the thresholds seen in Figure 4.66(B), 5-modes are required to account for $50 \%$ of the integrated turbulence kinetic energy and 14 POD modes are required to reach the $75 \%$ energy threshold. Components of the first five vectorial POD modes are shown in Figure 4.50. These are the modes showing the greatest organization and contributing to the largest structures in the turbulence field. In each subfigure, from left are the modes in increasing order (decreasing energy), and from top are the streamwise $\left(\phi_{x}^{(n)}\right)$, wall-normal $\left(\phi_{y}^{(n)}\right)$, and spanwise $\left(\phi_{z}^{(n)}\right)$ components of each POD mode. Color information is intentionally omitted from the figure as the POD modes themselves are unitless and do not represent energy. It is only in linear combination with their respective dynamic coefficients that the POD modes are able to express the fluctuating velocity field.

Figures 4.50(A) and 4.50(C) indicate that the near-wall behavior of the channel flow is represented in many POD modes. Although the near-wall turbulence length scales are smaller than those in the central region of the channel, the turbulence kinetic energy peaks near the wall. The POD requires many modes to fully describe this behavior. Large structures are seen in all three components of the POD modes away from the 

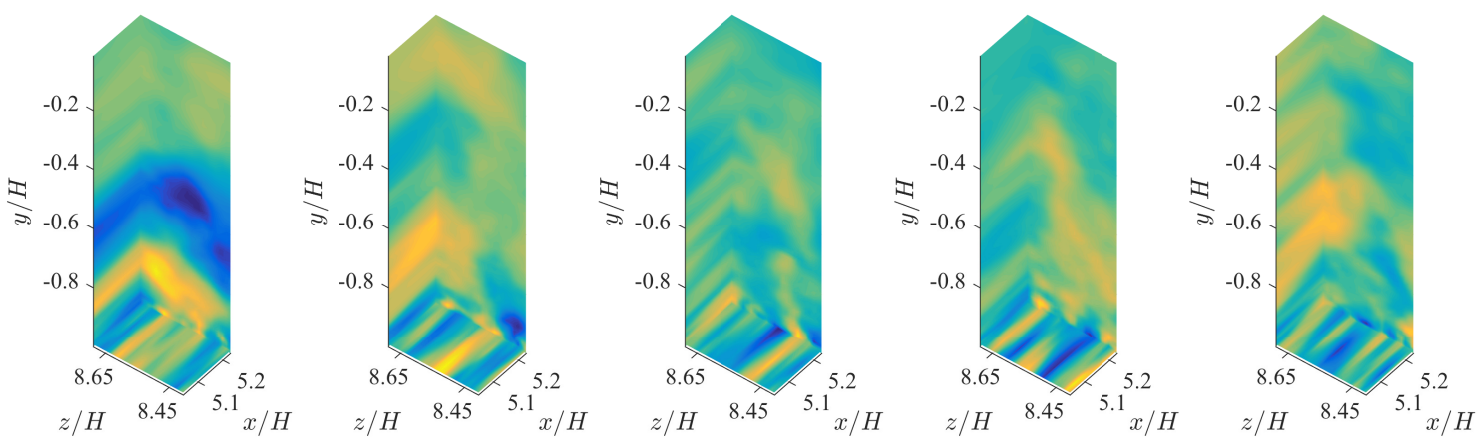

(A) $\phi_{u}^{(n)}$
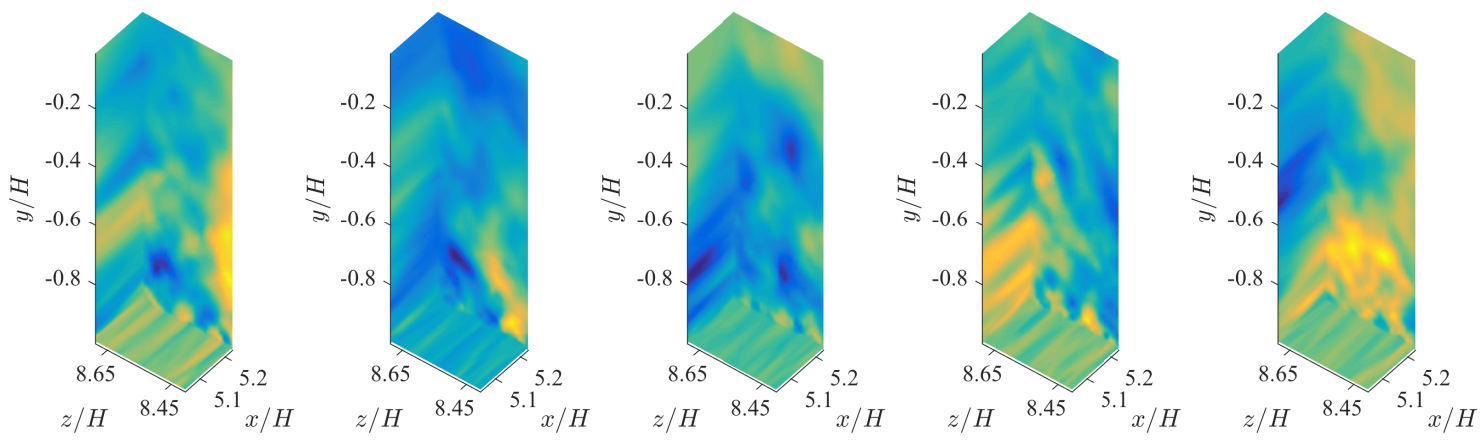

(B) $\phi_{v}^{(n)}$
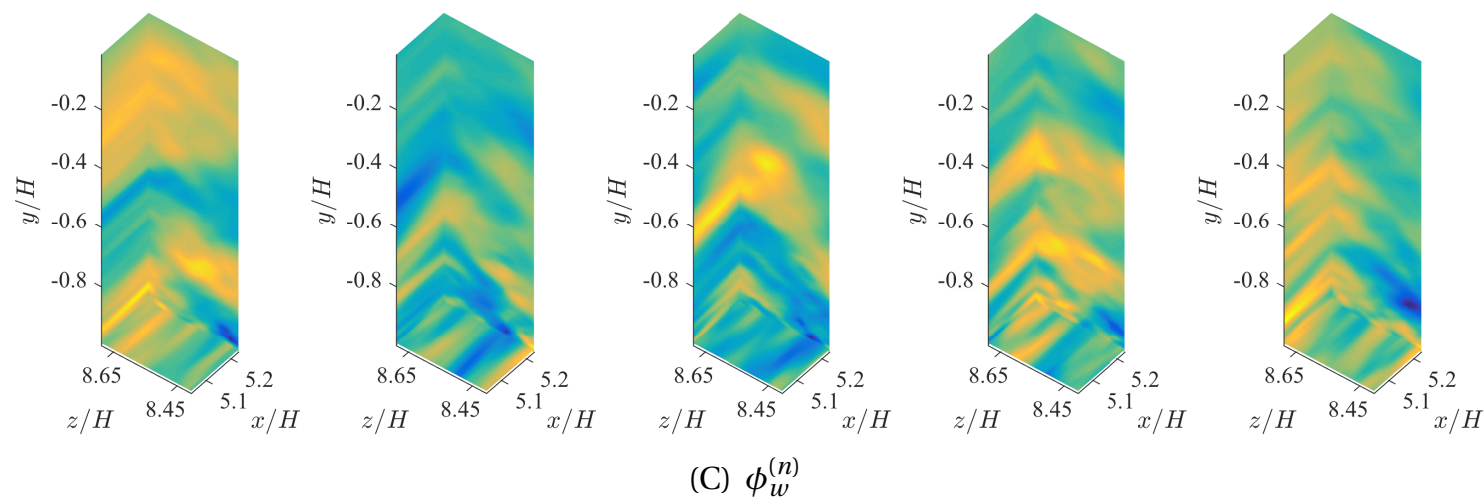

Figure 4.50: POD modes from channel flow volume. From left are modes 1 through 5.

channel wall. Behavior seen in Fourier modes, such as sinusoidal vibration modes, is not seen in the shown POD modes, indicating that the homogeneous turbulence is distributed through higher ranking modes. The low-rank POD modes accounting for the 
majority of the integrated turbulence kinetic energy are used to formulate low-order dynamical systems in the following sections. Modes shown in Figure 4.50 express the range of turbulence structures accounted for by the following dynamical system. An advantage to the POD is that modes are scaled based on energy contributed to the flow rather than time or length scales. This allows POD modes, in combination with their respective coefficients, to simultaneously contribute to the dynamics in the near-wall region and the center of the channel, provided that both regions are present in projections of the velocity field onto the POD subspace.

\subsubsection{Least-squares polynomial fit}

The dynamical system derived according to the formulation in section 2.7 is expected to account for instantaneous spanwise transport occurring in the channel flow, adding complexity to the resulting model. It is of interest to determine the capability of LODS to represent the full channel flow as a function of input modes. Mode bases analyzed in the following range between three modes (the minimum required to reconstruct 3D turbulence shown in Hamilton et al. ((submitted for publication March, 2016)) and 14 POD modes, corresponding to the $75 \%$ kinetic energy threshold.

The traditional Galerkin projection is not discussed at length here as the resulting dynamical systems function best in cases where turbulence is relatively low. Galerkin systems of channel flows have been undertaken in the past for transitional flow as in Aubry et al. (1988) and Ilak and Rowley (2008). In both cases, the Reynolds number is moderate $R e \sim 1000$. In the current study, the centerline Reynolds number $R e_{c} \sim$ $2.3 \times 10^{4}$ and the Galerkin projection requires quite a bit of tuning to be both stable and representative of the turbulence dynamics. A demonstration is included in B for 
planar (2D3C) data detailing that the Galerkin systems are highly dependent on the modes selected and prone to exaggerated damping.

Alternately, the low-order dynamical system pursued here follows the least-squares fit method. As such, the dynamical system predicting the behavior of the mode coefficients is derived from the derivatives of the coefficients from the POD. The leastsquares type LODS requires simultaneous realizations of both $a_{i}$ and $d a_{i} / d t$. Data sampled from the channel flow DNS has sufficiently high temporal resolution to yield accurate estimates of the time derivatives of the POD mode coefficients. In contrast, the Galerkin projection seeks these relationships through inner products of the mode basis and their projections onto the governing equations.

Although the parameters resulting from the least-squares fit (see Figure 4.51) do not arise from projection onto the governing equations, they are interpreted analogously where possible. The constant parameter $D_{i}$ quite small compared to the higher-order parameters, on the order of $10^{-6}$. Small magnitudes of $D_{i}$ occur according to the nature of the flow as being full-developed. Transient of spatially developing turbulence could be reflected by mode amplitudes that are not zero-centered, which the constant parameter would attempt to correct. In traditional Galerkin projection, the linear term $L_{i j}$ arises from the viscous term in the Navier-Stokes equations. The contribution to the viscous dissipation is largely accounted for by the smallest scales of turbulence, not accounted for in the truncated mode basis. $L_{i j}$ is the largest in magnitude of the fit parameters indicating a strong linear interaction between modes. It is also asymmetric about the diagonal where $i=j$, indicating that the mode-to-mode interactions in the dynamics of equation (2.40) are also asymmetric. For example, the largest magnitude of the parameter shown corresponds with $L_{1,5}$, implying that mode 5 is more 
dynamically relevant to the evolution of mode 1 than in the opposite case.

Quadratic parameters in $Q_{i j k}$ are taken to represent the combined convective and pressure terms. The pressure is not explicitly handled in the current dynamical system, although the effects of pressure fluctuations must be present in the velocity snapshots. In the interpretation of the Galerkin projection fixing $k=0$ is typically associated with convection; fixing $j=0$ is associated with the production term that evolves from Reynolds averaging of the convection term Navier-Stokes equations. In the LODS developed here, the mean flow field is omitted and there is no zeroth index. In the current case, there is not a zeroth POD mode as the mean flow is not considered in the dynamics. Instead the mode interactions are from turbulence only. Permuting the indices $j$ and $k$ in the quadratic term yields identical values; the interactions expressed by $Q_{i j k}$ are of mode $i$ with a combination of $j$ and $k$. Corresponding with the $50 \%$ threshold, 5 POD modes are used to compose the LODS seen in Figure 4.51.

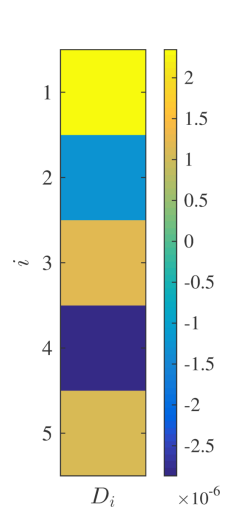

(A) $D_{i}$

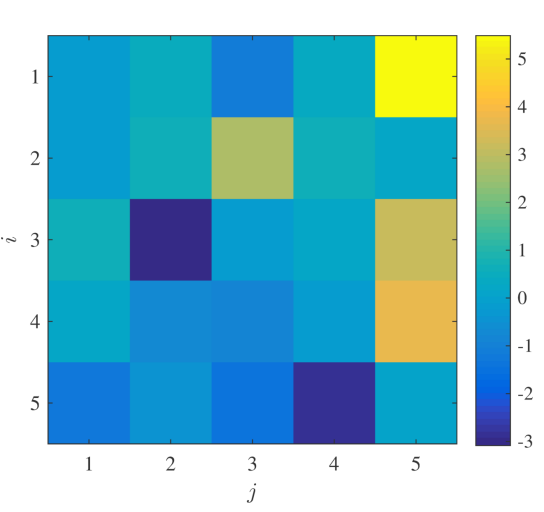

(B) $L_{i j}$

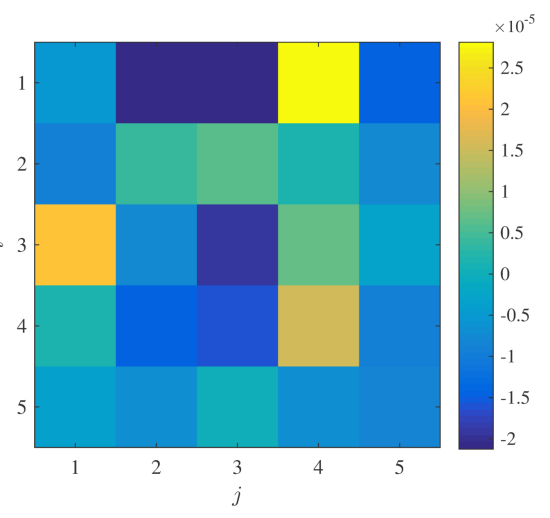

(C) $Q_{i j 1}$

Figure 4.51: Constant 4.51(A), linear 4.51(B), and quadratic 4.51(C) parameters from least-squares fit of $d \hat{a}_{i} / d t$. For the quadratic term shown, the index $k=1$ is fixed and other components are omitted. Cubic term is not shown for brevity.

Parameters shown in Figure 4.51 couple the mode coefficients $\hat{a}_{i}$ in the system of 
ODEs to be solved as in equation 2.40. The degree to which a mode is coupled to any others is shown as the relative magnitudes of each parameter. Extreme values imply strong correlation or anti-correlation in the dynamic evolution of the modes. Small values of the parameters are taken to indicate that a combination of the modes in question is negligible in the evolution of the system. The least-squares fit from equation 2.42 matches $d a_{i} / d t$ calculated from the time series of POD mode coefficients to one solved in the ODEs $d \hat{a}_{i} / d t$, shown in Figure 4.52(A) in black and blue lines, respectively. The figure demonstrates that the fit acts as a low-pass filter on $d a_{i} / d t$. Major features of the time derivatives are well-represented but many of the higher-frequency dynamics of $a_{i}$ are missed in the LODS.

Solving the ordinary differential equations results in predictions of coefficients $\hat{a}_{i}$ shown in Figure 4.52(B) as blue lines. Initial conditions are required for solutions, taken here as the first value of each coefficient from the POD, corresponding to $t=0$ from the channel flow DNS. The choice of initial conditions is arbitrary and the resulting approximations $\hat{a}_{i}$ evolve distinctly for each choice. This observation is exploited below to recalibrate the LODS and extend the predictions far past the input time. Deviations between the time derivatives of the POD coefficients and their respective least squares fits $\left(d a_{i} / d t\right.$ and $\left.d \hat{a}_{i} / d t\right)$ propagate through the solution of the ODEs seen clearly in the difference between $a_{i}$ and $\hat{a}_{i}$ in Figure 4.52(B). Although the fit of the derivatives is reasonable even at the highest shown mode number, error propagation through the coefficients is quite large. These errors are most notable in the highest modes used in the LODS, in this case modes 4 and 5, at the bottom of the figure. The coefficient $\hat{a}_{5}$ is nearly periodic and do not match the POD coefficients accurately. Increasing the modal basis used to compose the LODS naturally decreases the error be- 
tween the POD coefficients and the LODS equivalents. Error propagation beyond the input time of the sampled DNS grows quickly to the point where $\hat{a}_{i}$ eventually diverge and numerical solution is no longer possible. The ability to predict outside of the input time depends on the number of modes used to formulate the LODS and the error tolerance in the numerical solution of equation (2.40).

Figures 4.53 and 4.54 show the evolution of a similar LODS using 14 POD modes, corresponding to the $75 \%$ energy threshold. Least-squares fit parameters are not shown for brevity, but follow those shown in Figure 4.51. The time derivatives shown in Figure 4.53 demonstrate a tighter fit than in the 5-mode LODS, as expected. Error is reduced by allowing a greater range of mode coupling through the fit parameters. In the 14-mode LODS, significant deviations between $d a_{i} / d t$ and $d \hat{a}_{i} / d t$ are not seen until $n=12$. Increasing the mode basis used in the LODS and decreasing the error propagated through the ordinary differential equations naturally increases the ability of the LODS to predict the mode coefficients, shown in Figure 4.54. As with the 5mode LODS, error that propagates through the solution of the coupled ODEs is seen in the predicted coefficients $\hat{a}_{i}$. For consistency of comparison, the LODS is restricted to making predictions with the input time.

To quantify the goodness of fit between the POD and LODS coefficients, the RMS span error is calculated between the coefficients $a_{i}$ and $\hat{a}_{i}$, and their time derivatives $d a_{i} / d t$ and $d \hat{a}_{i} / d t$. Values are normalized by the span of the POD coefficients are shown in tables 4.1 and 4.2 calculated as,

$$
\text { span err }=\left|\frac{\left(a_{i, \max }-a_{i, \min }\right)-\left(\hat{a}_{i, \max }-\hat{a}_{i, \min }\right)}{\left(a_{i, \max }-a_{i, \min }\right)}\right| \times 100 \%
$$



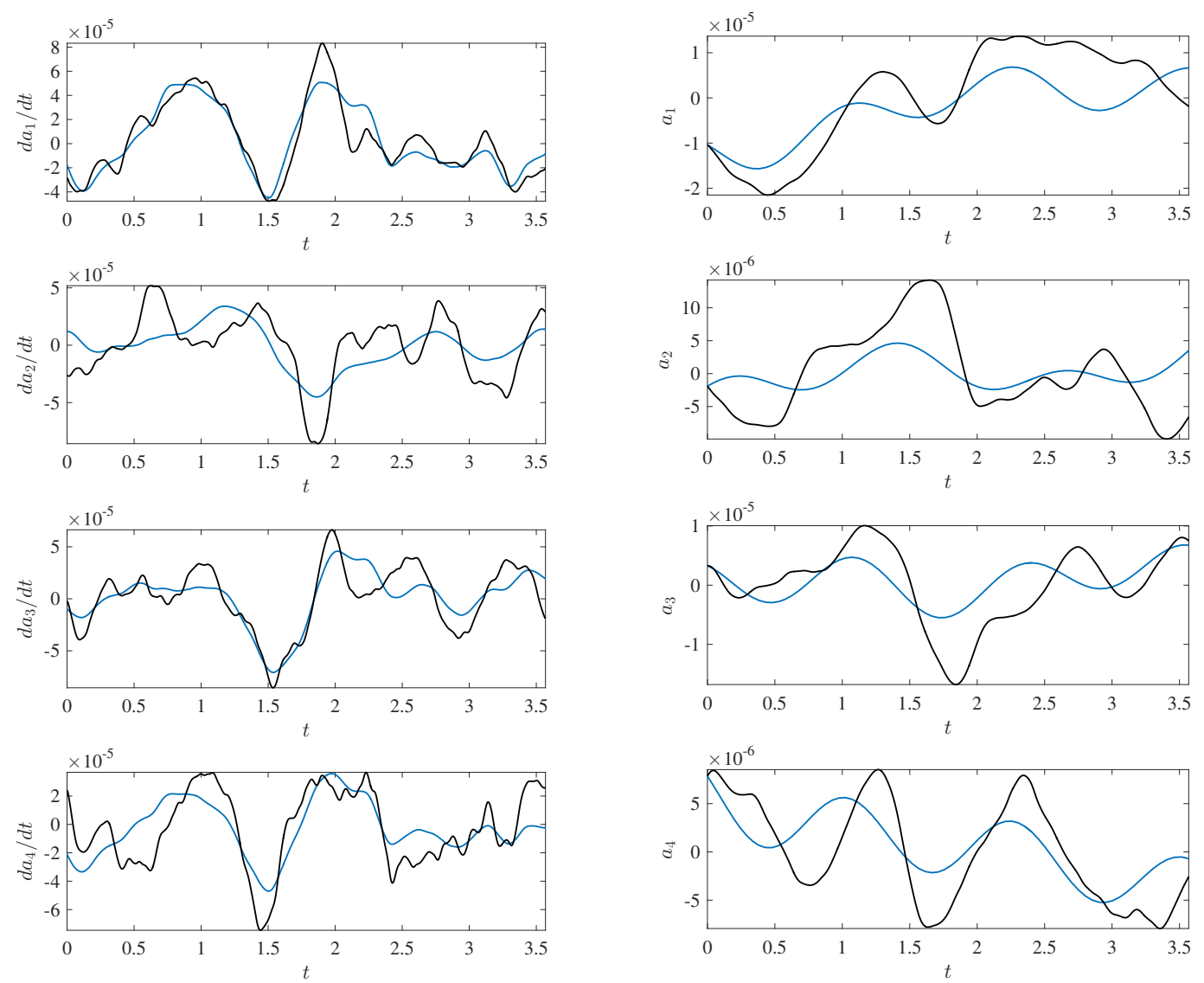

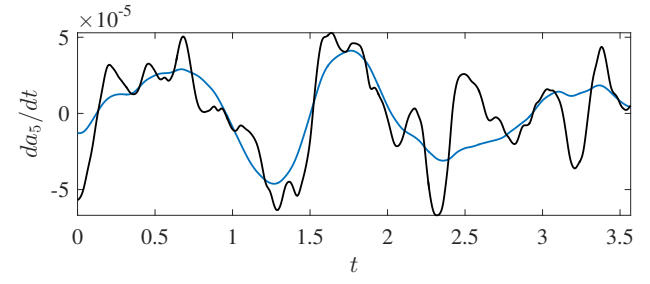

(A) $d a_{i} / d t$ vs. $d \hat{a}_{i} / d t$

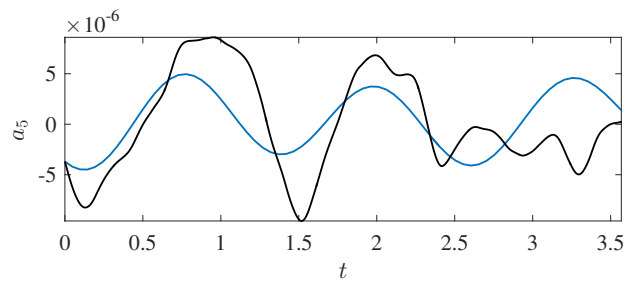

(B) $a_{i}$ vs. $\hat{a}_{i}$

Figure 4.52: Time derivatives of POD mode coefficients (black) and their respective LODS approximations (blue).

Quantitative comparisons between the POD and LODS are termed RMS differences, rather than RMS errors, which are saved for comparisons between the sampled DNS statistics and either the POD or LODS. Coefficients evolving from the LODS have a resolution in time that is a function of the numerical tolerance of the solver. In order to 

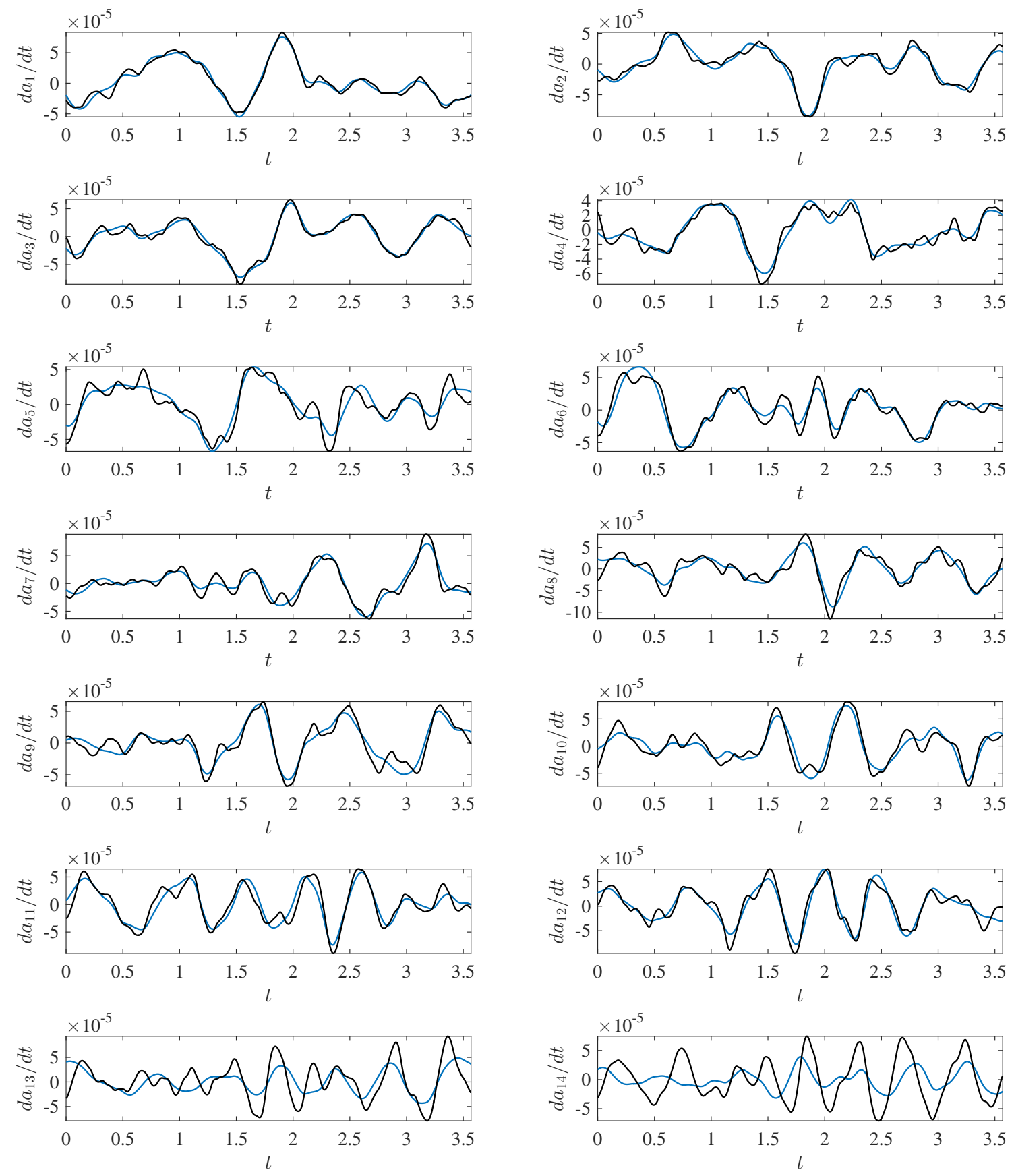

Figure 4.53: Time derivatives of POD mode coefficients (black) and their respective LODS approximations (blue). 

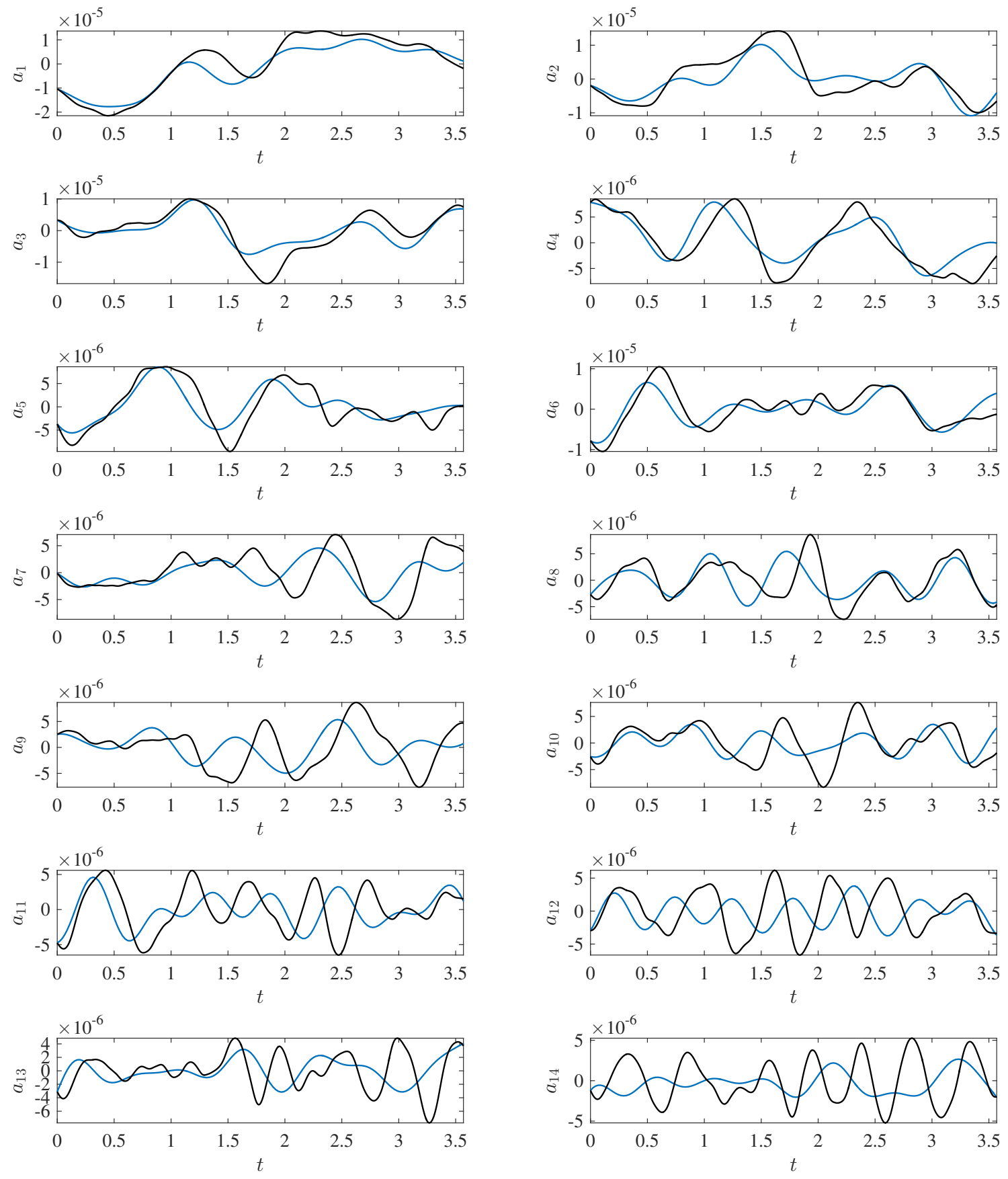

Figure 4.54: POD mode coefficients (black) and their respective LODS approximations (blue) calculated from the time derivatives shown in Figure 4.53. 
Table 4.1: Normalized RMS error difference $d a_{i} / d t$ and $d \hat{a}_{i} / d t$ according to the number modes $N_{r}$ used in the LODS.

\begin{tabular}{|c|c|c|c|c|c|c|c|c|c|c|c|c|c|c|}
\hline & Mode 1 & 2 & 3 & 4 & 5 & 6 & 7 & 8 & 9 & 10 & 11 & 12 & 13 & 14 \\
\hline$N_{r}=3$ & 72.8 & 45.8 & 49.3 & - & - & - & - & - & - & - & - & - & - & - \\
\hline 4 & 70.9 & 42.8 & 50.1 & 74.2 & - & - & - & - & - & - & - & - & - & - \\
\hline 5 & 26.8 & 42.6 & 23.3 & 25.6 & 27.1 & - & - & - & - & - & - & - & - & - \\
\hline 6 & 27.0 & 31.5 & 23.1 & 14.9 & 26.4 & 48.9 & - & - & - & - & - & - & - & - \\
\hline 7 & 27.1 & 28.3 & 26.6 & 13.5 & 24.5 & 46.0 & 82.1 & - & - & - & - & - & - & - \\
\hline 8 & 9.8 & 8.0 & 11.1 & 15.6 & 22.5 & 34.9 & 81.3 & 65.0 & - & - & - & - & - & - \\
\hline 9 & 4.2 & 1.7 & 9.0 & 14.0 & 8.9 & 31.6 & 36.3 & 50.1 & 26.2 & - & - & - & - & - \\
\hline 10 & 4.1 & 2.3 & 9.9 & 12.6 & 9.0 & 30.2 & 15.0 & 37.2 & 23.4 & 24.6 & - & - & - & - \\
\hline 11 & 4.3 & 3.0 & 8.0 & 8.4 & 8.8 & 1.0 & 14.8 & 39.5 & 23.9 & 17.2 & 52.0 & - & - & - \\
\hline 12 & 0.8 & 3.3 & 12.9 & 8.9 & 0.3 & 0.0 & 14.9 & 23.8 & 19.4 & 8.0 & 21.2 & 27.5 & - & - \\
\hline 13 & 0.7 & 1.2 & 12.8 & 9.1 & 1.5 & 2.5 & 13.8 & 25.8 & 15.7 & 12.1 & 22.3 & 22.4 & 52.1 & - \\
\hline 14 & 0.7 & 3.3 & 12.0 & 8.8 & 1.0 & 2.3 & 13.7 & 24.8 & 11.2 & 11.9 & 14.0 & 11.4 & 46.4 & 51.1 \\
\hline
\end{tabular}

make difference calculations, the predicted coefficients $\hat{a}_{i}(\hat{t})$ are interpolated to the original time input $t$. Interpolation of the derivatives is not necessary as they are calculated to fit the input time by definition. Reflecting the trends seen in Figures 4.52(A) and 4.53, the error associated with the derivative of any particular mode coefficient time derivative decreases as a function of the number of modes used in the truncated POD basis $N_{r}$. It is not always the case, however, that the normalized RMS difference increases with $\mathrm{n}$ for a fixed basis $N_{r}$; there many cases in which a higher ranking mode exhibits less error than a low rank mode within a given basis.

RMS difference between the coefficients and their LODS predictions is less organized than for the derivatives. In a general sense, one can say that the coefficients predicted by the LODS will decrease with $N_{r}$, indeed the limit of the RMS error must be zero as $N_{r} \rightarrow N$, but the decrease in error does not appear to be monotonic. For example, the minimum normalized RMS difference between $a_{1}$ and $\hat{a}_{1}$ occurs for $N_{r}=9$. These trends reflect that the mode interactions described by the LODS parameters do not follow a monotonic increase or decrease, and certain mode interactions may lead to a decrease in LODS accuracy. RMS errors for $\hat{a}_{i}$ and $d \hat{a}_{i} / d t$ are shown in Figure 4.55 
Table 4.2: Normalized RMS difference between $a_{i}$ and $\hat{a}_{i}$ according to the number modes $N_{r}$ used in the LODS.

\begin{tabular}{c|cccccccccccccc} 
& Mode 1 & 2 & 3 & 4 & 5 & 6 & 7 & 8 & 9 & 10 & 11 & 12 & 13 & 14 \\
\hline$N_{r}=3$ & 69.4 & 68.9 & 70.1 & - & - & - & - & - & - & - & - & - & - & - \\
4 & 69.9 & 57.5 & 61.8 & 19.3 & - & - & - & - & - & - & - & - & - & - \\
5 & 36.0 & 70.6 & 54.3 & 20.6 & 48.1 & - & - & - & - & - & - & - & - & - \\
6 & 16.1 & 21.6 & 31.6 & 3.6 & 24.5 & 34.4 & - & - & - & - & - & - & - & - \\
7 & 19.2 & 33.8 & 18.6 & 13.5 & 24.1 & 36.5 & 55.8 & - & - & - & - & - & - & - \\
8 & 13.6 & 22.2 & 39.4 & 5.3 & 10.9 & 37.9 & 34.8 & 49.8 & - & - & - & - & - & - \\
9 & 9.5 & 17.5 & 28.1 & 4.9 & 24.0 & 41.7 & 32.5 & 53.2 & 25.5 & - & - & - & - & - \\
10 & 23.2 & 19.1 & 30.6 & 2.3 & 21.1 & 36.6 & 44.3 & 54.2 & 43.7 & 49.1 & - & - & - & - \\
11 & 13.1 & 36.3 & 34.3 & 13.6 & 20.6 & 39.3 & 42.4 & 68.8 & 41.0 & 68.7 & 24.8 & - & - & - \\
12 & 14.6 & 27.1 & 22.4 & 5.0 & 15.0 & 27.6 & 23.5 & 47.7 & 25.6 & 31.9 & 23.9 & 29.9 & - & - \\
13 & 19.5 & 46.4 & 46.1 & 7.2 & 26.1 & 22.7 & 50.3 & 34.3 & 48.4 & 58.4 & 17.1 & 44.3 & 27.6 & - \\
14 & 20.5 & 12.7 & 35.7 & 13.0 & 22.2 & 28.6 & 36.8 & 35.5 & 36.8 & 54.1 & 22.7 & 41.2 & 42.7 & 55.1
\end{tabular}

for the 14-mode LODS.

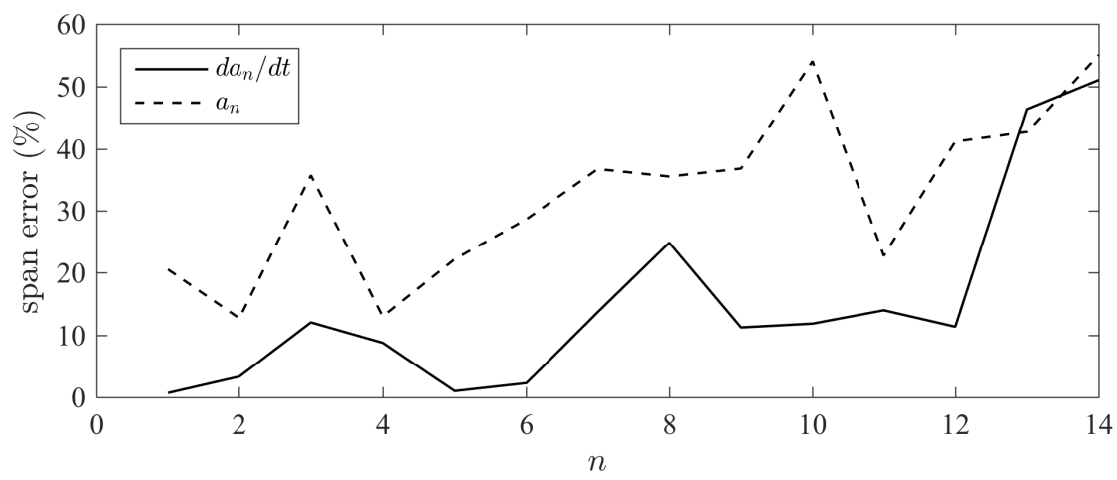

Figure 4.55: RMS difference of mode coefficients and their time derivatives (normalized by span) between the POD and the LODS. Error shown corresponds with the $N_{r}=14$ rows of tables 4.1 and 4.2 .

\subsubsection{Periodic LODS recalibration}

Ideally, the LODS is able to make predictions of the velocity field at arbitrarily times in the future. However, in practice the numerical solutions presented above propagate error through to $\hat{a}_{i}$ that compiles and, at long solution times, causes the system to grow unstable and diverge. The dynamics determined by the parameters $D_{i}, L_{i j}, Q_{i j k}$, and $C_{i j k l}$, specify mode interaction; the trajectory of the system also requires initial 
conditions, above taken as $a_{i}(t=0)$. Using different initial conditions produces distinct coefficient evolutions that obey the dynamics specified by the LODS parameters. The sensitivity of the LODS to supplied initial conditions is used as a means to periodically recalibrate the solution, resulting in a system capable of making predictions at arbitrarily long times.

Recalibration is undertaken by matching the value of a single LODS coefficient at the end of the integration time $\hat{a}_{\text {rand }}\left(t=t_{f}\right)$ to its respective POD coefficient. The coefficient to be matched is selected randomly to guarantee that the LODS does not fall into a loop, which occurs when any single mode is used for recalibration. The time at which the discrepancy $\left|a_{\text {rand }}\left(t_{m}\right)-\hat{a}_{\text {rand }}\left(t_{f}\right)\right|$ is minimized is denoted as the 'matching time' $t_{m}$ and is taken to be within the input time range $0 \leq t_{m} \leq t_{f}$. The initial conditions are refreshed using the POD coefficients at the matching time $a_{i}\left(t_{m}\right)$, and a new solution to equation (2.40) is calculated. The resulting time span for $M$ periods of LODS recalibration is $0 \leq t \leq M \times t_{f}$. Recalibration of the LODS limits the error propagation in the coefficients at the cost of introducing discontinuities in $\hat{a}_{i}$.

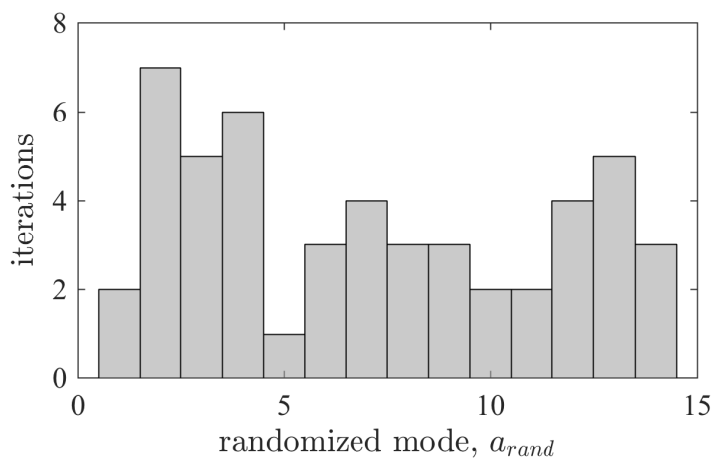

(A)

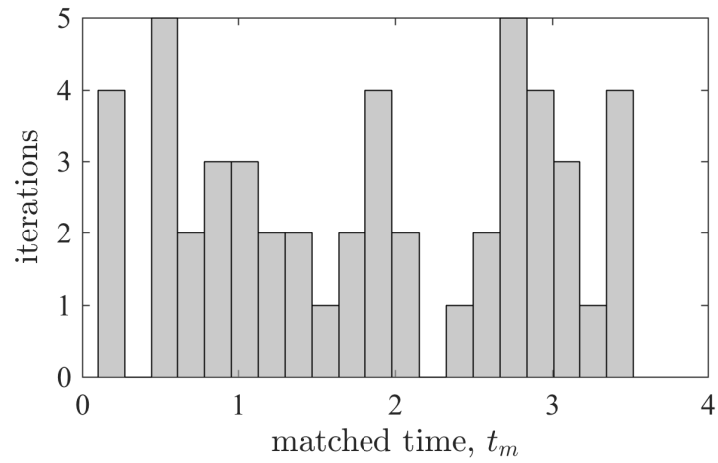

(B)

Figure 4.56: Histograms depicting 4.56(A) mode selected in periodic recalibration and 4.56 (B) index selected by matching coefficients. 
The distribution of the mode coefficient $a_{\text {rand }}$ and the matched time $t_{m}$ are seen in Figures 4.56(A) and 4.56(B). Because the mode number is selected randomly, the distribution of $a_{\text {rand }}$ tends toward uniformity as the number of recalibrations $M$ increases. The matching time $t_{m}$ is a function of the specific value of $a_{\text {rand }}$, and the distribution in Figure 4.56(B) is less intuitive. LODS coefficients with $M=50$ periodic recalibrations are seen in Figure 4.57, demonstrating that $\hat{a}_{i}$ remains bounded in the neighborhood of $a_{i}$. The predicted coefficients fill a vector space defined by the truncated POD basis to a greater degree than record of their respective POD coefficients, suggesting that the LODS is capable of accounting for dynamics outside of the input snapshot basis.

Figure 4.58 compares the streamwise normal stress calculated from the input snapshot basis, the reduced order description from the truncated POD basis, and the periodically recalibrated LODS, respectively from left. Only the streamwise normal stress is visualized as it is the dominant contributor to the turbulence kinetic energy. As anticipated, the POD estimate of $\overline{u u}$ (Figure 4.58(B)) picks out the large scale and energetic features of the statistically derived Reynolds stress shown in Figure 4.58(A). Large structures below $y / H=-0.5$ are captured successfully by the POD, including features very near the wall shown at the bottom of each subfigure. Features captured by the LODS in Figure 4.58(C) are similar to the POD estimates. Differences between the POD and LODS estimates are seen most clearly in the near-wall region, where the LODS produces streaks more closely matching the original statistics.

Comparing the streamwise/wall-normal Reynolds shear stress $\overline{u v}$ as in Figure 4.59, it is evident that the LODS makes predictions not seen by the POD estimate. Figure 4.59(C) predicts a second structure of high magnitude on the left side of the shown volume. This structure is not present in either the POD or the sampled statistics, show- 

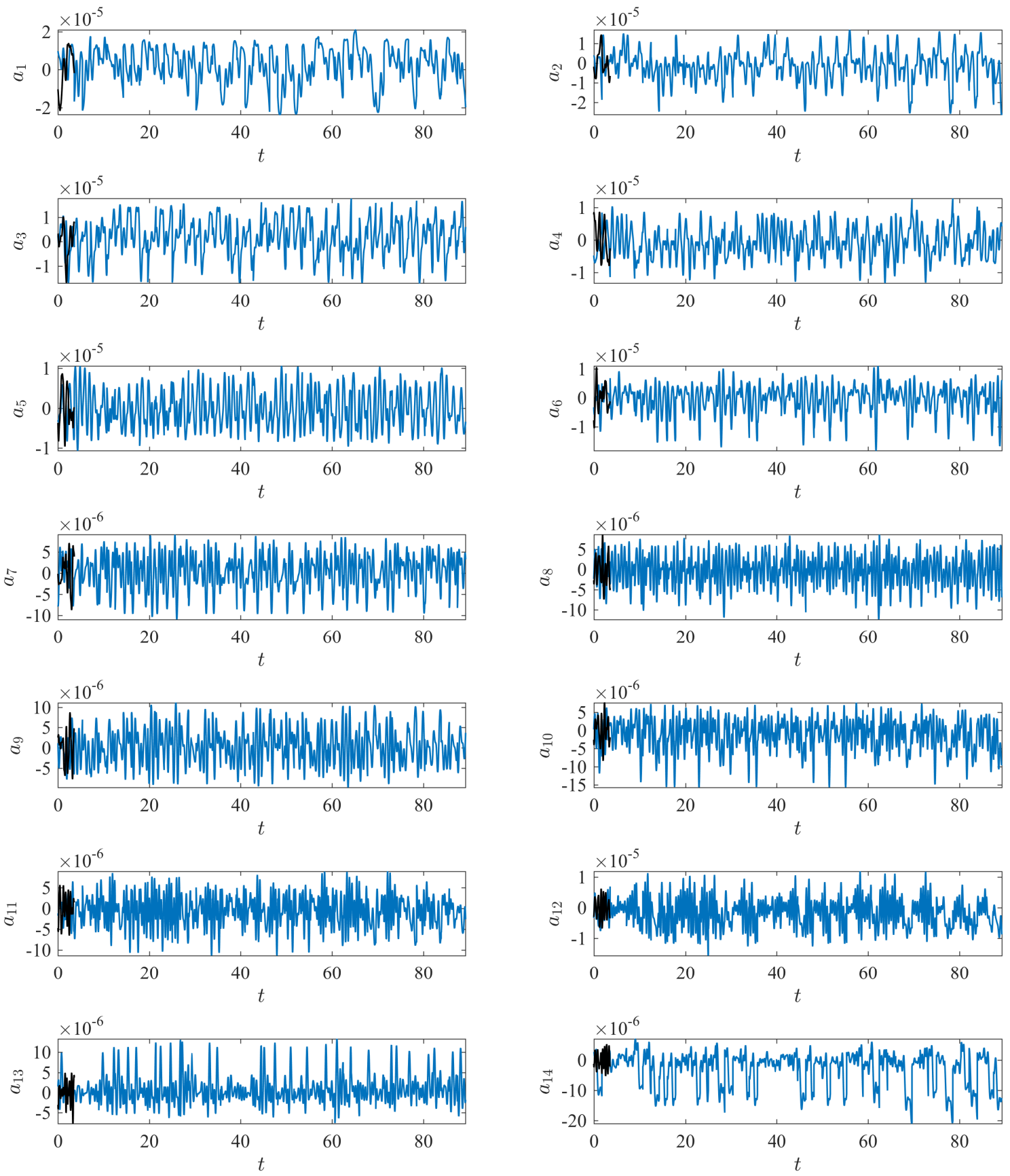

Figure 4.57: POD mode coefficients (black) and their respective LODS approximations (blue) calculated from the time derivatives shown in Figure 4.53. 


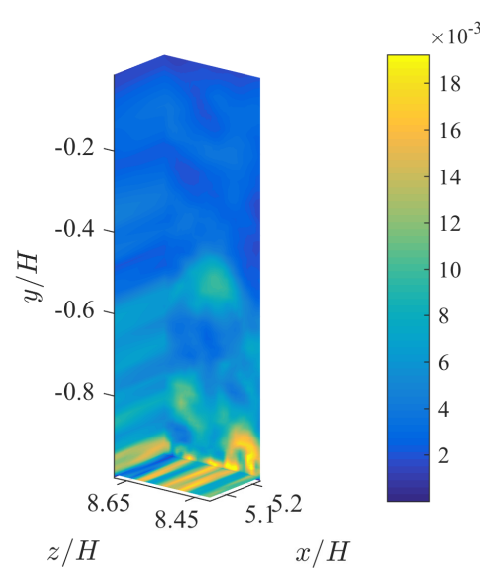

(A) $\overline{u u}$

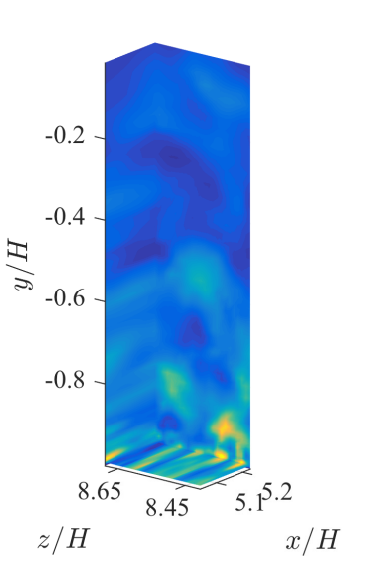

(B) POD estimate of $\overline{u u}$.

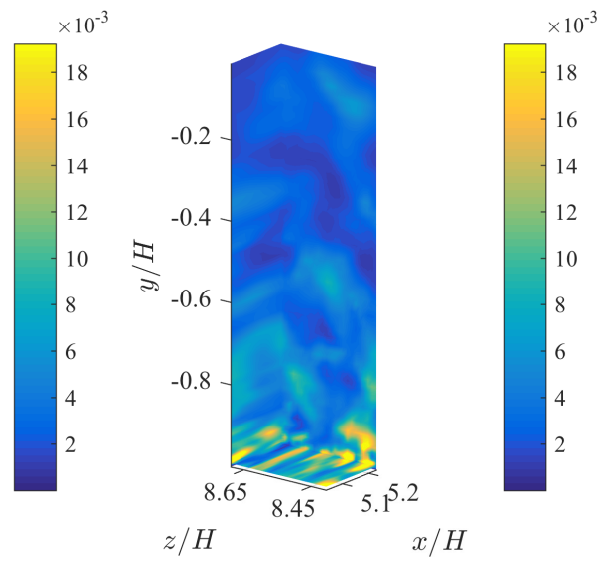

(C) LODS estimate of $\overline{u u}$.

Figure 4.58: Comparison of streamwise normal Reynolds stress from sample statistics, POD reconstruction, and LODS estimate, from left.

ing that the model is predicting outside of the input dynamics. Both the POD and the LODS exaggerate regions where $\overline{u v} \leq 0$ for $y / H \geq-0.6$; the POD and the LODS have a difficult time reproducing the tendency toward null values far from the wall. The superposition (and interaction, in the case of the LODS) of a large basis of modes is necessary to converge toward null values of the stresses far from the wall.

In order to benchmark the performance of the LODS, profiles of spatially averaged quantities are compared in Figure 4.60. In each subfigure, a single component of the Reynolds stress tensor is averaged in the streamwise and spanwise directions, denoted with angle brackets $\langle\cdot\rangle$. Subfigures compare the spatially averaged stress for the full channel flow DNS as a dashed black line, reproduced from Graham et al. (2013), with the profiled from the sampled statistics (black lines), the truncated POD basis (orange), and the LODS (blue). From the figure, it is clear that the LODS makes estimates of the Reynolds stresses in the channel that are closer to the sample statistics (and the full DNS) than the POD. The wider range of prediction in the LODS is a product of the 


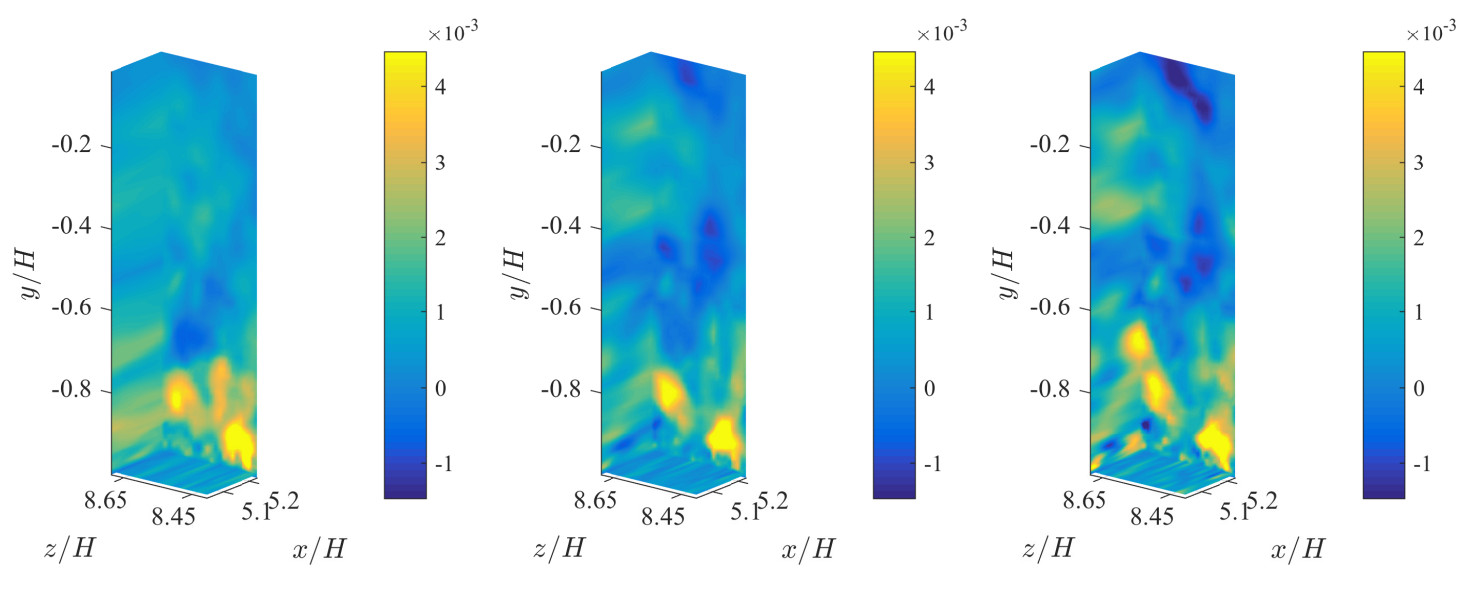

(A) $\overline{u v}$

(B) POD estimate of $\overline{u v}$.

(C) LODS estimate of $\overline{u v}$.

Figure 4.59: Comparison of streamwise/wall-normal Reynolds shear stress from sample statistics, POD reconstruction, and LODS estimate, from left.

longer time record from periodic recalibration and the filling out of the state space of the coefficients according to the relationships defined by the LODS parameters. Increased performance is clear in the near-wall values of Reynolds normal stresses in Figure 4.60(A) through Figure 4.60(C).

The performance of the LODS is not as easily distinguished in terms of the turbulence shear stresses. For example, the LODS profile of $\langle\overline{u v}\rangle$ makes a better estimate near the wall and toward the center channel than the sampled statistics do and performs approximately as well as the statistics in the range of $-0.8 \leq y / H \leq-0.4$. Spatially averaged shear stresses $\langle\overline{u w}\rangle$ and $\langle\overline{v w}\rangle$ tend toward zero with perfect statistical convergence and are typically excluded in channel flow discussions as spanwise fluctuations are less important than those in the streamwise and wall-normal directions. The dashed lines in Figures 4.60(E) and 4.60(F) are set to identically zero, and do not come from the DNS documentation. Profiles of the LODS and POD are consistent with the sampled statistics near the wall but deviate in the range of $-0.8 \leq y / H \leq-0.4$, es- 


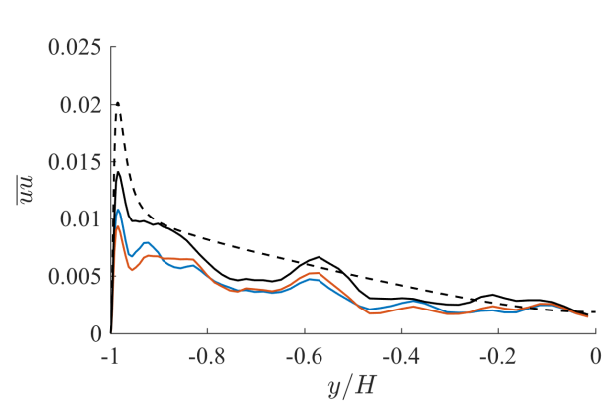

(A)

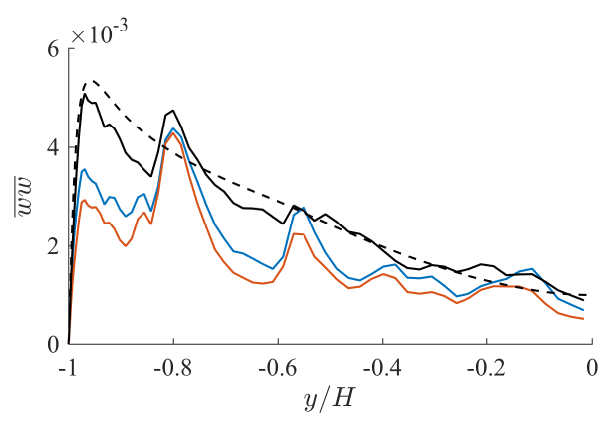

(C)

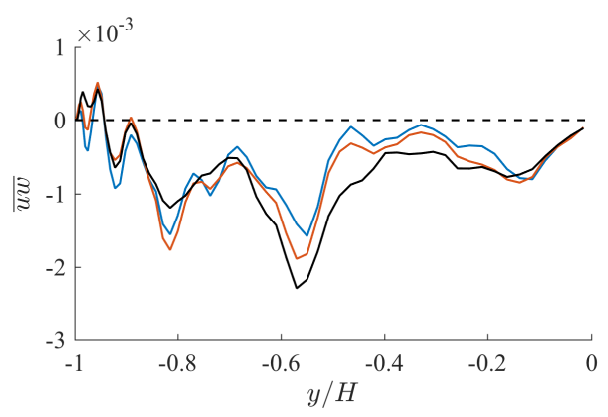

(E)

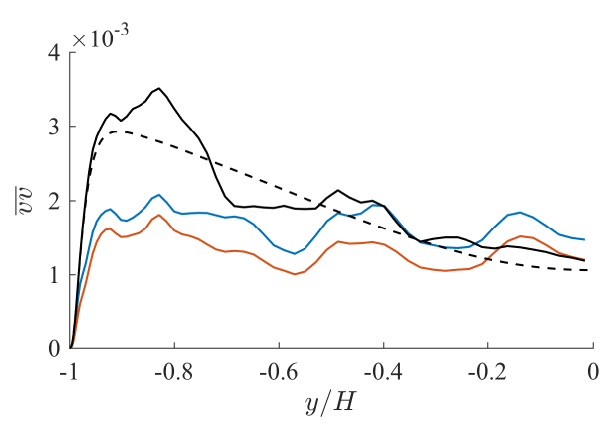

(B)

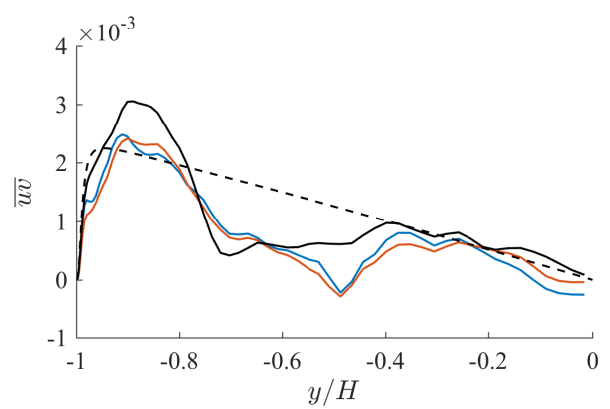

(D)

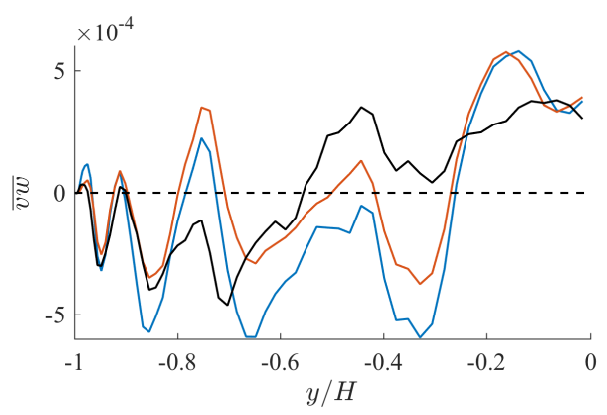

(F)

Figure 4.60: Wall-normal profiles of Reynolds stresses comparing statistics of sampled data (black), POD reconstructions (orange), and LODS with periodic recalibration (blue), and the spatially averaged profile from the full DNS (black,dashed).

pecially noticeable in $\langle\overline{v w}\rangle$, where the LODS is significantly farther from the zeros line than the statistics or the POD.

Normalized RMS span errors according to equation (4.18) are calculated for the 
Table 4.3: Span error (\%) between $\overline{u_{i} u_{j}}$ between the sampled turbulence field and approximations from the POD or the LODS with periodic recalibration.

\begin{tabular}{c|cccccc} 
& $\overline{u u}$ & $\overline{v v}$ & $\overline{w w}$ & $\overline{u v}$ & $\overline{u w}$ & $\overline{v w}$ \\
\hline POD & 33.8 & 48.5 & 15.9 & 11.8 & 11.4 & 13.2 \\
LODS & 15.5 & 34.4 & 15.3 & 12.2 & 10.7 & 67.1
\end{tabular}

spatially averaged profiles shown in Figure 4.60, detailed in Table 4.3. Quantifying the deviation between the spatially averaged profiles of $\overline{u_{i} u_{j}}$ and the estimates from the reduced order descriptions indicates that the LODS represents error reduction up to $18 \%$ over the POD. The greatest reduction in the span error is seen for $\overline{u u}$. Error shown for the shear stresses $\overline{u v}$ and $\overline{u w}$ are very similar to those of the POD. However, looking at $\overline{v w}$, the error increases moving from POD description to LODS estimate, reflecting the trend seen in Figure 4.60(F). The LODS describes the evolution of the 14 most energetic POD modes, including interactions not seen in the time series of the coefficients $a_{n}$. Because the spanwise/wall-normal stress is the smallest in magnitude of the stress tensor, it is most sensitive to deviations from the POD orbit.

Dynamic characteristics of the sampled channel flow turbulence and the low-order approximations are shown through energy spectra in Figures 4.61 and 4.62 for two wall-normal locations, $y^{+}=29.89$ and $y^{+}=371.6$, respectively. The constrained sample domain prohibits the calculation of wavenumber spectra without significant convolution error. However, as the sampled data LODS (shown with and without periodic recalibration) have sufficient time resolution and relatively long records, spectra as functions of frequency are within reach. Profiles shown have been averaged over the 24 points sampled along the spanwise coordinate. In the case of periodic recalibration, the velocity signals are significantly longer in time than the sample from the channel flow DNS, allowing for lower frequencies to be resolved and increasing the 
resolution in $f$. Recalibrating the LODS as undertaken above matches a single mode coefficient at a prescribed time interval. There, the matched mode coefficient is continuous in time, but the other coefficients experience a discontinuity. Abrupt changes in the LODS coefficients $\hat{a}_{i}$ propagate through to the velocity fields and into the spectra causing scatter, particularly significant at frequencies $f \geq 50 \mathrm{~Hz}$. Noise introduced to the spectra by discontinuities in the velocity signals are overcome by breaking the long velocity signals into blocks increasing the number of realizations over which averaging is performed.

The POD organizes contributions to the basis according to energy, and individual modes may account for energy from a range of frequencies as seen in the spectra. Spectra shown for the LODS in Figures 4.61(B) and 4.61(B) reflect the filtering effects of truncating the POD basis to 14 out of 550 modes. Magnitudes of the LODS spectra match those of the sample data, but the form is significantly altered, particularly for high frequencies. To some degree, the filtering effect is mitigated by periodic recalibration; the flattened range seen in Figures 4.61(C) and 4.62(C) is shorter than for the dynamical system without recalibration. The streamwise energy spectrum for $y^{+}=371.6$ show more energy in the lowest frequencies than the original data, indicating that the LODS selectively increases fluctuations in the streamwise velocity in center of the channel. The large bump seen in all spectra around $f \approx 20 \mathrm{~Hz}$ likely arises from the nonlinear filtering of the POD. Spectra from the LODS with periodic recalibration are clearly quite different than the sample statistics but represent an improvement over the estimate of the system without recalibration. 


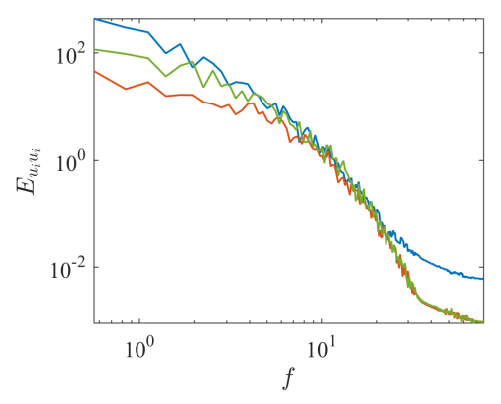

(A) Sample statistics

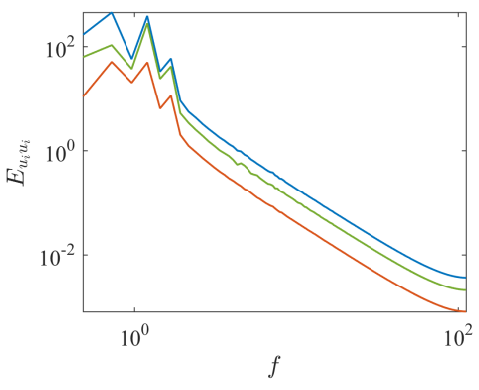

(B) LODS

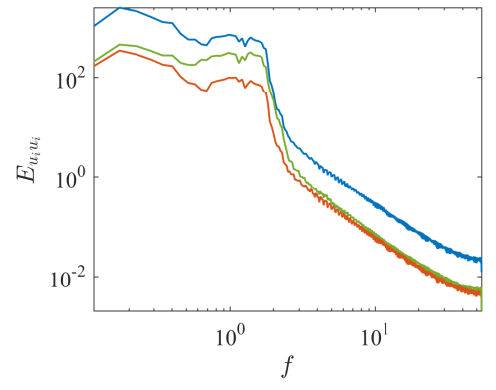

(C) LODS with periodic recalibration

Figure 4.61: Frequency spectra of the fluctuating velocity field at $y^{+}=29.89$. In each subfigure are spectra of streamwise velocity $\left(E_{u u}\right.$, blue), wall-normal velocity ( $E_{v v}$, orange), and spanwise velocity ( $E_{w w}$, green).

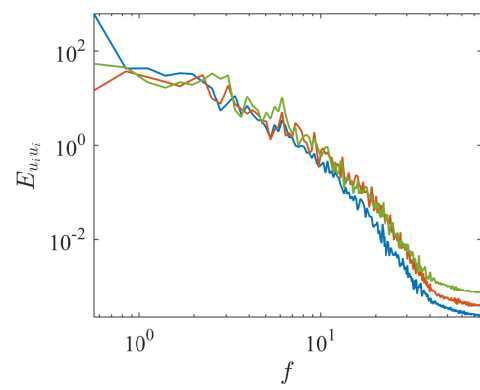

(A) Sample statistics

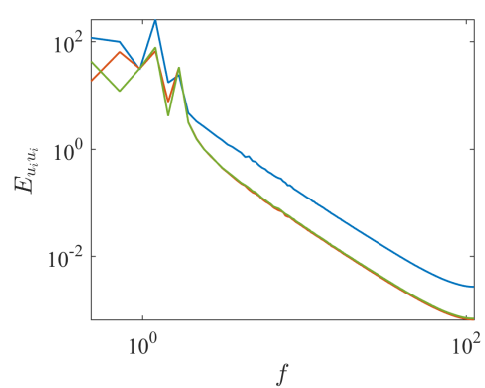

(B) LODS

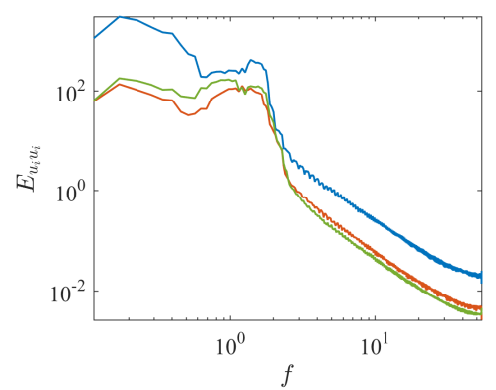

(C) LODS with periodic recalibration

Figure 4.62: Frequency spectra of the fluctuating velocity field at $y^{+}=371.6$. In each subfigure are spectra of streamwise velocity $\left(E_{u u}\right.$, blue), wall-normal velocity ( $E_{v \nu}$, orange), and spanwise velocity ( $E_{w w}$, green).

\subsubsection{Conclusions}

Reduced order dynamical systems are formulated for the fully-developed turbulent channel flow DNS hosted at Johns Hopkins University. Results indicate that the Galerkin projection in its given formulation is not capable of capturing the dynamics of the channel flow in a stable system without considerable tuning. Previous work in similar conditions (Aubry et al., 1988) showed Galerkin systems that were signifi- 
cantly more able to describe the flow only by limiting the complexity of the flow by constraining it to transitional turbulence.

Fully-developed turbulence at high Reynolds number has proved to be resistant to the Galerkin projection in almost every case (see e.g. 2.6). However, other forms of dynamical systems have proven to be considerably more functional, including deriving the system of coupled ODEs through the least-squares fit method. The LODS demonstrated above combines the time-dependent POD mode coefficients directly in formulating a dynamical system, in contrast with the Galerkin projection, which instead seeks a dynamical system through the inner product of the Navier-Stokes equations with the proper orthogonal modes. Parameters resulting from the LODS are analogous to those from the GS; in addition to the linear and quadratic terms in the Galerkin system, there are constant and cubic terms, which add both complexity and stability to the dynamics.

The LODS composed here for the channel flow does not arise from a mathematical projection onto the governing behavior law of the flow. While this may appear to limit the physical significance of the dynamical system, it also adds generality. By removing the physical meaning associated with each set of parameters, they become more flexible, in that they may take on a range of values that might appear inconsistent with the behavior laws governing the flow. At the same time, extending an analogy to the linear and quadratic parameters provides an estimate of the influence of viscous or convective terms in the LODS.

Deviation between the LODS and DNS statistics is expected; spatially averaged profiles of the Reynolds stresses demonstrate that the low-order dynamical system can reproduce the turbulence fairly accurately. However, the dynamics included in the 
system are necessarily confined to those outlined by the POD, and ultimately the dynamics included in the sampled basis of velocity snapshots. The sample used to compose the LODS represents a small slice of the full simulation; the full range of dynamics and turbulence structures was not included in the kernel of the POD and cannot be reproduced by the LODS. By increasing the dynamics accounted for in the kernel of the POD, the reduced order model above is more able to match statistics drawn from the full simulation. With greater representation of the dynamics from the DNS, the spectra of the velocities produced by the dynamical systems would better represent the full flow but would still be subject to filtering from truncating the POD mode basis. A limitation to the LODS developed here is the need for simultaneous realizations of both the mode coefficients and their respective time derivatives, which is difficult to achieve with many experimental techniques. Time-resolved PIV and a dual-time PIV are methods that have been employed in the past (Perret et al., 2006b; D'adamo et al., 2007) to accurately develop time derivatives of the POD mode coefficients.

The low-dimensional model explored here is capable of predicting velocity snapshots that are only slightly outside the range of the dynamics input in the POD mode coefficients. Broadening the sample data will lead to better statistical convergence of the input modes and, provided that accurate estimates of $d a_{i} / d t$ are obtained, will certainly lead to better approximation of the full DNS statistics. A thorough analysis of the system response to perturbations and input functions would advance the control capabilities of low-dimensional models like those composed here. Correlating an upstream signal with the POD coefficients would provide a more intuitive recalibration method, leading to accurate reflections of inflow disturbances. Control models of these sorts would be attractive for a variety of aerodynamics and flow control applica- 
tions. 


\subsection{Reduced-order model of a wind turbine wake}

The mean flow field is shown in Figure 4.63, where the momentum deficit area of the wake is visualized as the blue areas in $U$ (Figure 4.63(A)). The data in Figure 4.63 are ensemble averaged over the full range of the LES, resulting in proper statistical convergence of mean velocities. Accordingly, mean velocities change minimally outside of the region of the wake. In all subfigures, transparency has been employed to aid in visualization. In figures demonstrating wake statistics, either mean velocities or Reynolds stresses, the transparency has been adjusted to emphasize the streamwise evolution of the given quantity.

Wall-normal and spanwise mean velocities ( $V$ and $W$, respectively) show activity only very close to the modeled turbine rotor, consistent with the experiments in Section 4.1 detailing phase dependence of turbulence structures very near the wind turbine rotor. Tangential forcing is applied at the rotor in order to include rotational effects seen in experimental data. Both the spanwise and wall-normal mean velocities are approximately symmetrically distributed in the near wake and range between $\pm 1.75 \mathrm{~m} / \mathrm{s}$. Magnitudes of $V$ and $W$ are quite small except very near the wind turbine rotor.

Second order wake statistics characterizing the turbulence field are shown in Figures 4.64 and 4.65. Reynolds normal stresses are positive by definition, and the transparency of the figures has been adjusted to focus on areas where magnitudes are high. The streamwise normal stress $\overline{u u}$ shows peak values of approximately $5.75 \mathrm{~m}^{2} / \mathrm{s}^{2}$ trailing the rotor at the height of the top tip of the disk $(z / D \approx 1.5)$. Wake statistics for a single wind turbine in isolation evolve more slowly in the streamwise direction than they would for a wind turbine in a large array. Here the peak values of $\overline{u u}$ develop at 


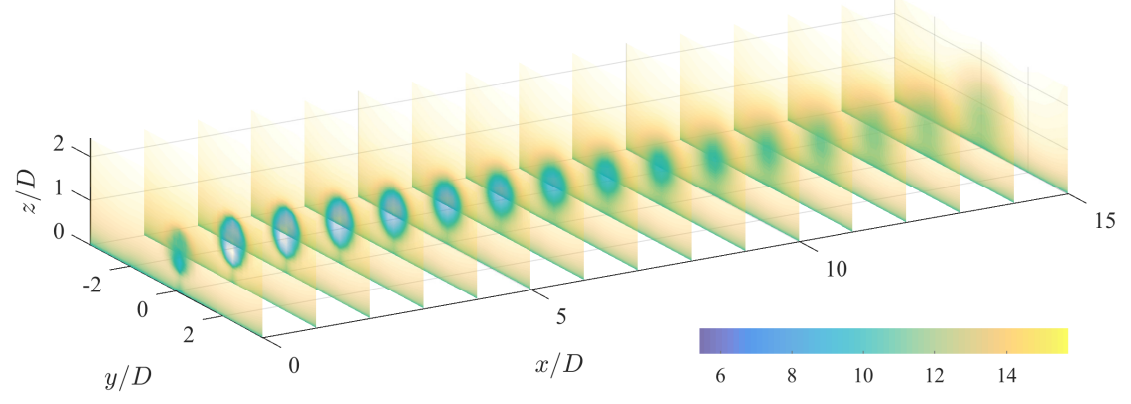

(A) $U$

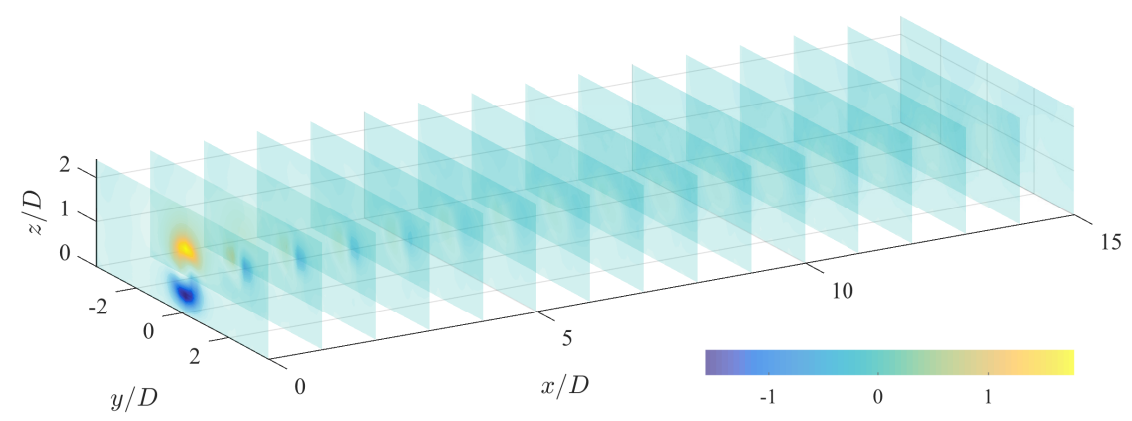

(B) $\mathrm{V}$

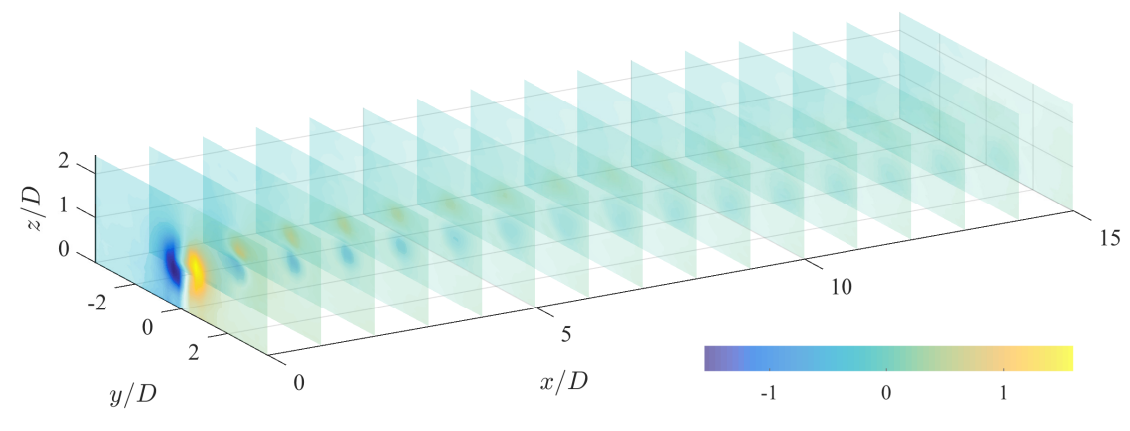

(C) $W$

Figure 4.63: Mean velocity in the wake of an isolated wind turbine.

$x / D \approx 6$. Although slower to develop along $x / D$, the turbulence field shares features with the growing body of work for wind turbine arrays. The streamwise normal stress is nearly null following the nacelle of the turbine and below hub height. As the field evolves downstream, the contour plots shown $\overline{u u}$ growing trailing the swept area of the edge of the rotor disk, mainly above hub height. 
Fluctuations in the streamwise are larger than those in the spanwise and wallnormal directions, leading to higher values of $\overline{u u}$ as compared to $\overline{v v}$ and $\overline{w w}$, which both remain below $1.5 \mathrm{~m}^{2} / \mathrm{s}^{2}$. The spanwise normal stress $\overline{v v}$ shows two regions of large magnitude, immediately above and below hub height. Wall-normal Reynolds stresses are characterized by as single region of large magnitude that develops centered on the hub of the rotor disk. As with the streamwise stress, $\overline{v v}$ and $\bar{w}$ reach peak values deep into the wake, in the range of 7 and 10 rotor diameters for the spanwise and wall-normal Reynolds stresses respectively.

Turbulence shear stresses developing in the wake show more interesting, if expected, trends in the wind turbine wake. In the case of a single wind turbine in isolation, inertial terms from the mean kinetic energy equation describing the wake flow are expected to dominate in the wake. Accordingly, shear terms are smaller in magnitude than the Reynolds normal stresses. Flux of mean flow kinetic energy into the momentum deficit area of the wake is assisted by the shear stresses but, unlike the wake in a large array, turbulent flux is not expected to be a first order term in the wake. Rather, shear stresses are expected to be most significant in higher-order wake statistics found in the turbulence kinetic energy transport equation, for example. Figure 4.65(B) shows the Reynolds shear stress $\overline{u w}$, corresponding to the covariance of the streamwise and spanwise velocity fluctuations. This term of the Reynolds stress tensor contributes to later fluxes of mean kinetic energy and is equal in peak magnitudes to the spanwise and wall-normal shear stresses. Similarly, the shear term $\overline{u v}$ assists in vertical transport of mean flow kinetic energy. Positive values of $\overline{u v}$ occur below hub height and correspond to upward flux; negative values occur above hub height and correspond to flux downward from above the wake. Curiously, Figure 4.65(A) indicates that there is a 


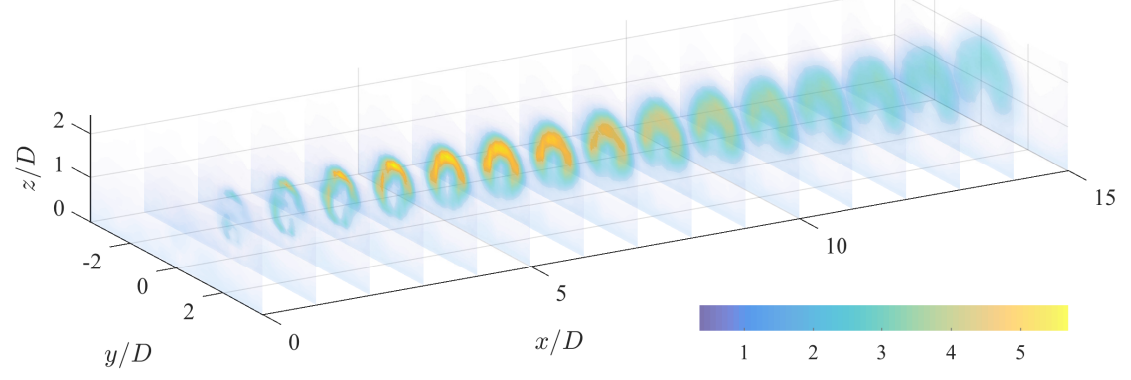

(A) $\overline{u u}$

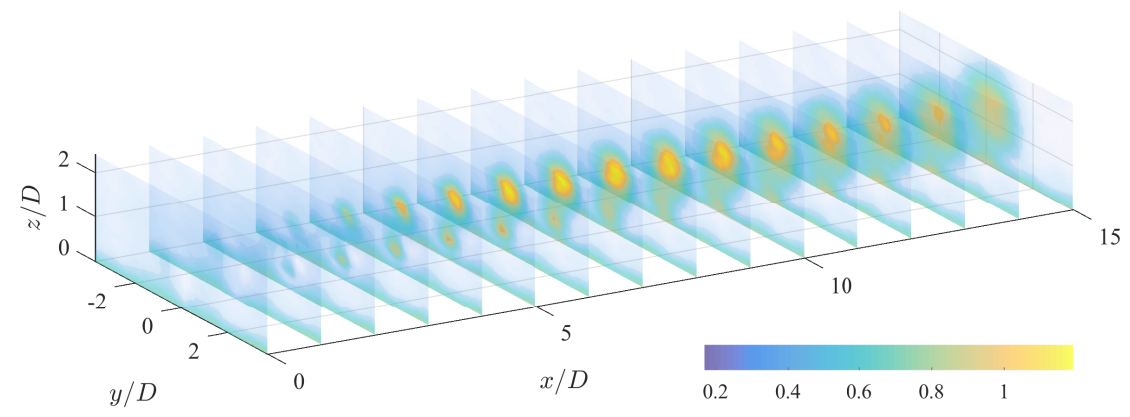

(B) $\overline{v v}$

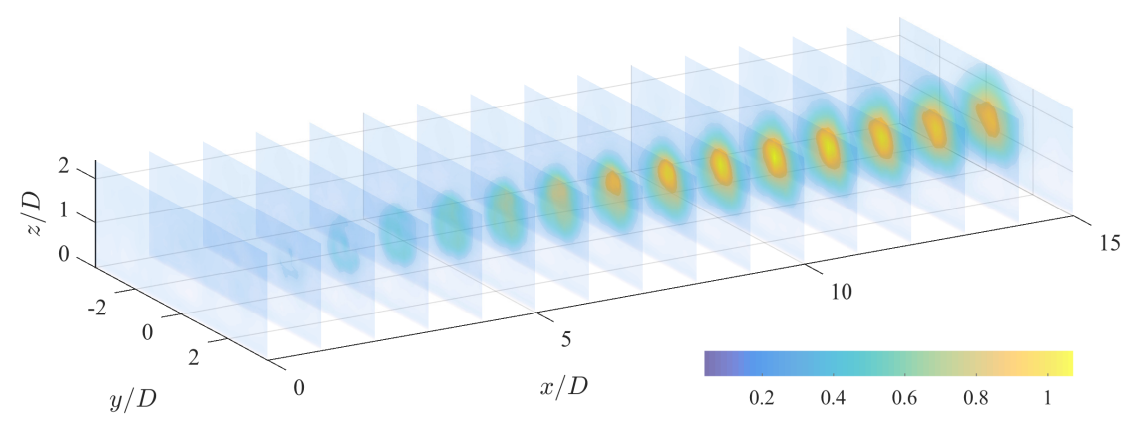

(C) $\overline{w w}$

Figure 4.64: Reynolds normal stresses.

lag between the peak upward and downward fluxes of mean flow energy into the wake. The spanwise/wall-normal stress is an order of magnitude smaller than the other components, resulting from the near axisymmetry seen in the turbulence field.

Proper orthogonal decomposition is applied to the LES wake data taking each snapshot as a full volume of the fluctuating velocity field. The two-point correlation 


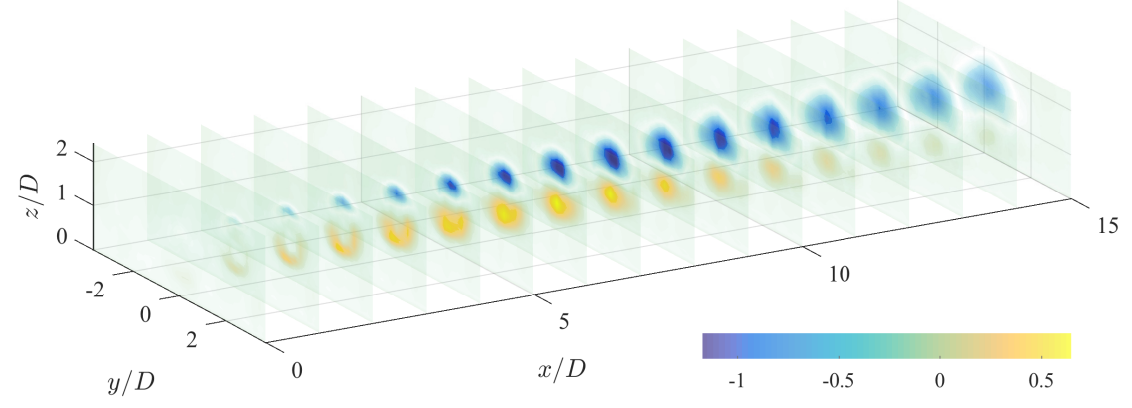

(A) $\overline{u v}$

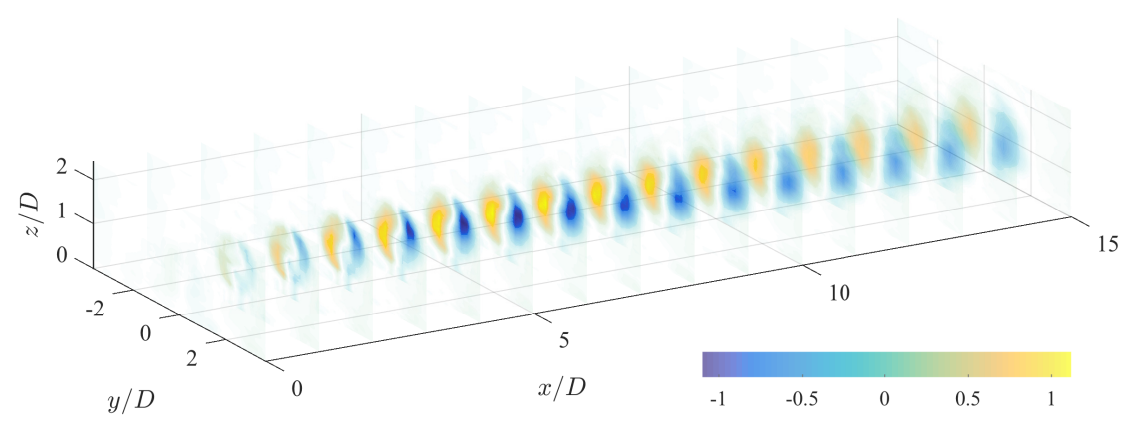

(B) $\overline{u w}$

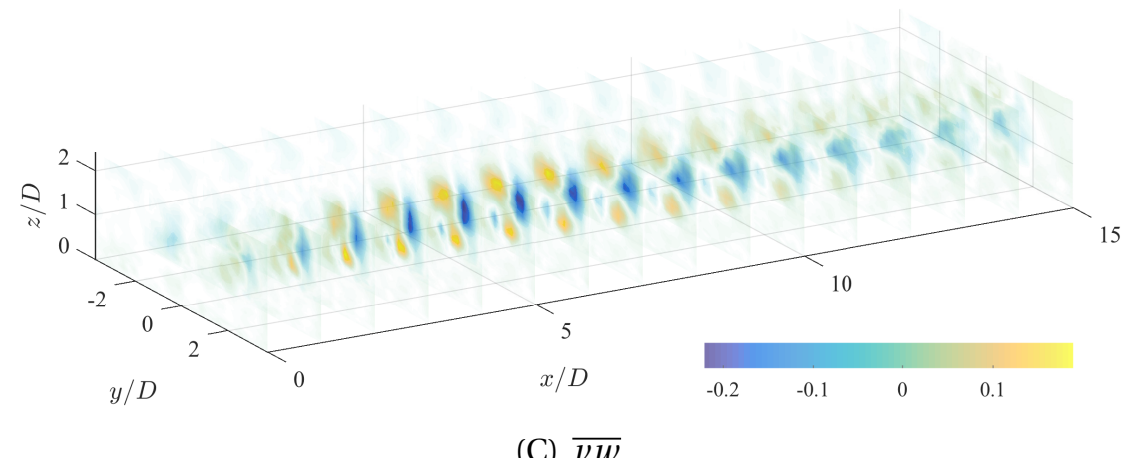

Figure 4.65: Reynolds shear stresses.

tensor forming the kernel of the decomposition effectively accounts for the turbulence field characterized by the Reynolds stresses seen in Figures 4.64 and 4.65. The eigenvalues of the POD from Equation (2.16) are shown in Figure 4.66(A), normalized by the integrated turbulence kinetic energy of the volume. Application of the POD to LES data shows slow accumulation of energy by mode due to periodicity in the simulation 
space. In the case where the kernel of the POD is homogeneous in space, POD modes reduce to Fourier modes, which is less efficient in accounting for energy in the system. The nature of the subgrid filtering and periodic boundary conditions in the LES introduce homogeneity that slow the rate of convergence of $\lambda_{n}$. Cumulative summation of eigenvalues $\lambda_{n}$, shown in Figure 4.66(B), are used to establish the portion of energy represented by a truncated basis of POD modes. Energy thresholds selected for basis truncation is arbitrary but needs to include enough modes to capture the prevailing dynamics of the turbulence. The particular number of modes used in low-dimensional reconstruction of turbulence stress fields is undertaken as,

$$
\overline{u_{i}(\boldsymbol{x}) u_{j}(\boldsymbol{x})}=\sum_{n=1}^{N_{r}} \lambda^{(n)} \phi_{i}^{n}(\boldsymbol{x}) \phi_{j}^{n}(\boldsymbol{x}),
$$

where $N_{r}$ is the number of modes retained in the truncated basis. The cumulative summation of eigenvalues in Figure 4.66(B) indicates the percentage of the total energy represented by a given number of modes. The full mode basis (equal to the number of input snapshots $N=2000$ ) is required to represent $100 \%$ of the turbulence kinetic energy.

Figure 4.67 demonstrates example modes from the POD basis. Only the streamwise component is shown and only for the first three modes. The domain in each subfigure has been rotated from the demonstrations of the other statistics to better illustrate the spatial complexity of each mode. Color scale information is excluded as the modes do not carry physical units. The streamwise component of modes 2 and 3 show features of apparently similar scale translated (or perhaps more appropriately, phase shifted) in the streamwise direction. Similarity in structures communicated by the modes arise from two sources: snapshots are not statistically uncorrelated in time and periodicity 


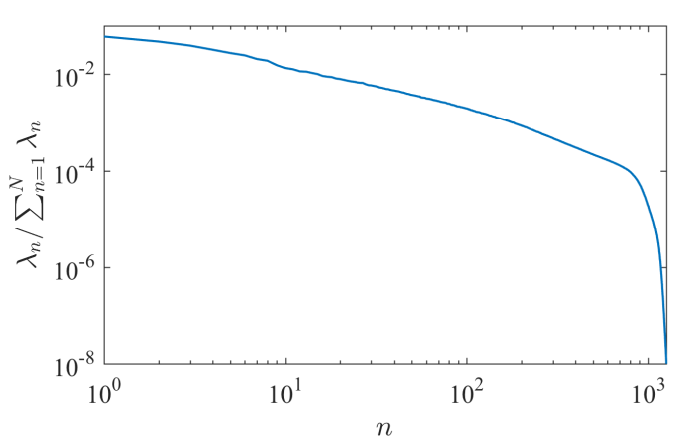

(A)

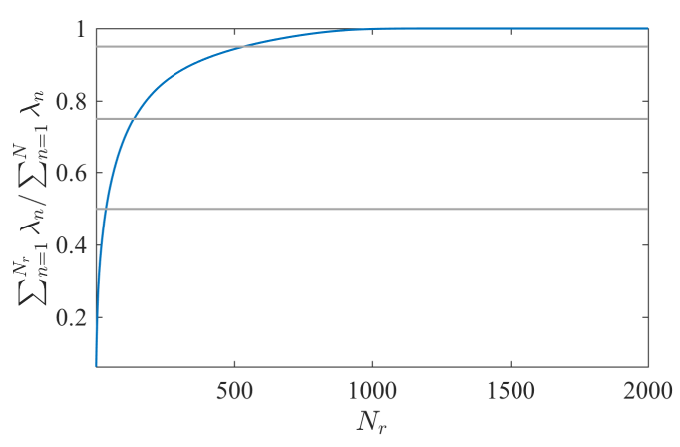

(B)

Figure 4.66: Distribution of energy in the POD mode basis according to eigenvalues $\lambda_{n}$. Normalized values appear in (a); the normalized cumulative summation of eigenvalues is shown in (b) with thresholds corresponding to 50, 75 and $95 \%$ of the integrated turbulence kinetic energy in the domain.

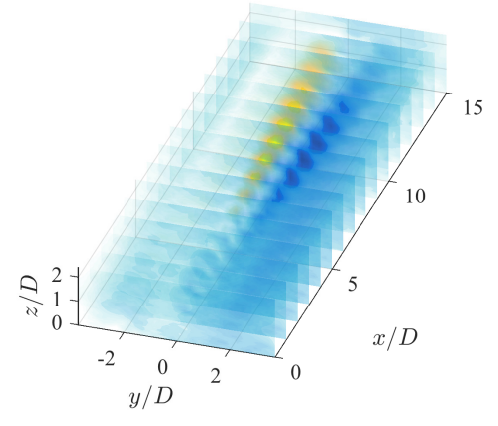

(A) $\phi_{u}^{(1)}$

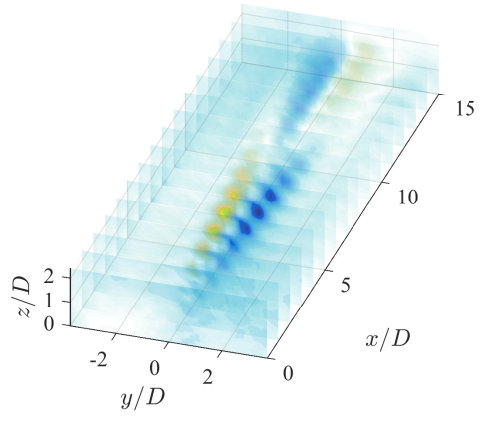

(B) $\phi_{u}^{(2)}$

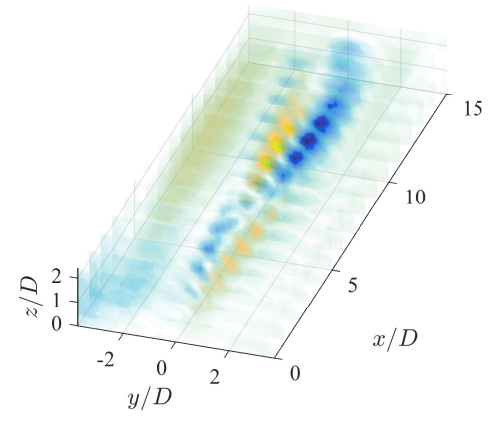

(C) $\phi_{u}^{(3)}$

Figure 4.67: Example POD modes from wind turbine wake used in low-order model.

inherent to LES data. These effects cause some structures in the input snapshots to be overrepresented by the POD and appear in more than one mode. Mode pairing (referred to as sister modes) are commonly seen in POD applied to time resolved data and do not affect the methods ability to represent the turbulence field, but certainly slow the convergence of energy contained in the eigenvalues.

Back projecting the modes onto the snapshot basis and integrating over the do- 


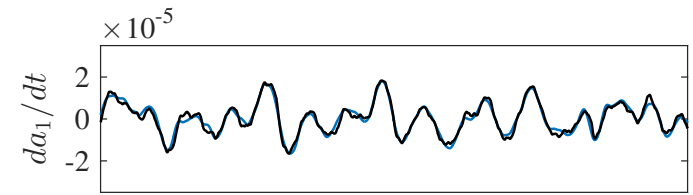

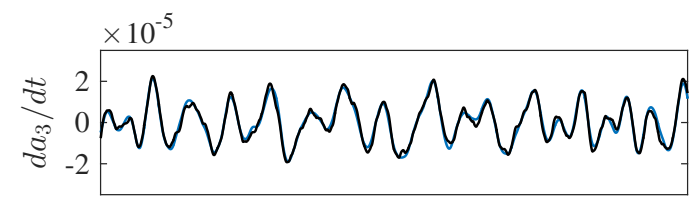

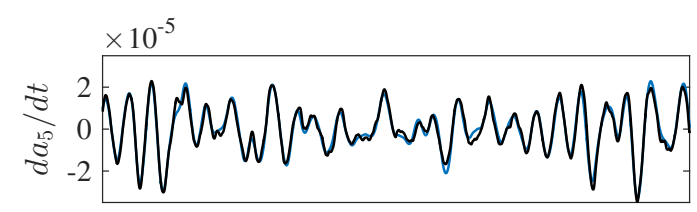

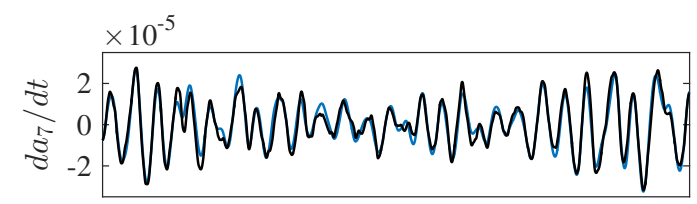

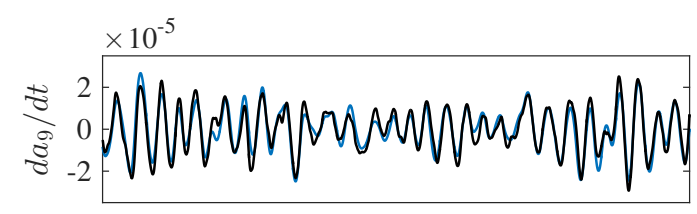

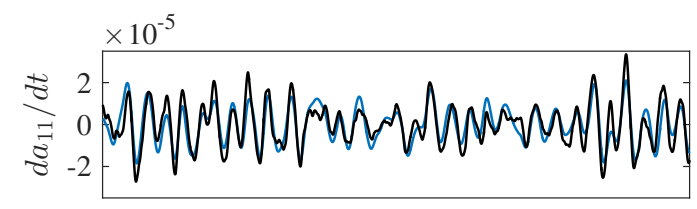

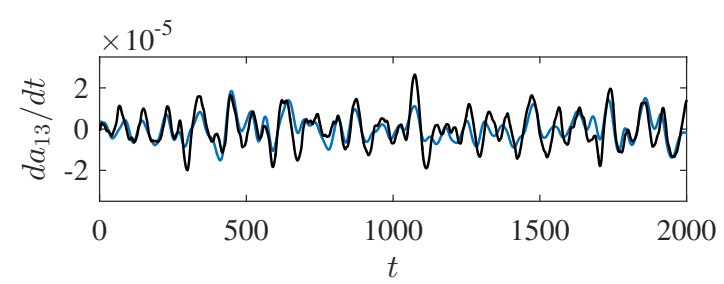

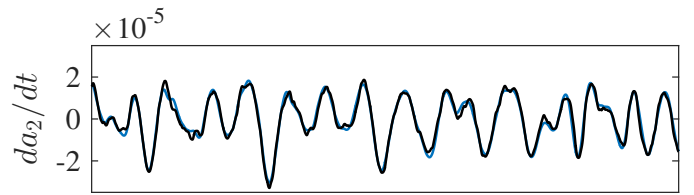

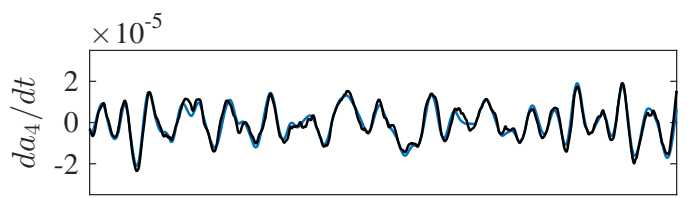

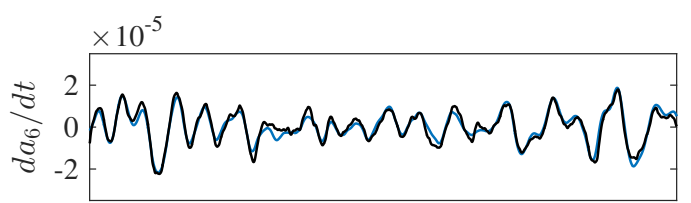

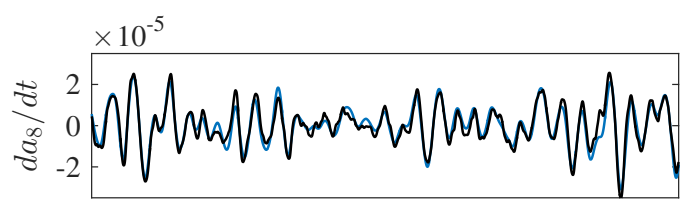

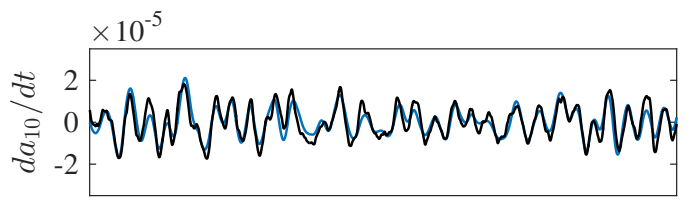

5.

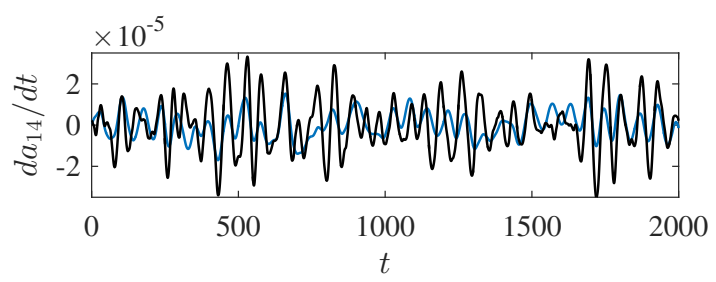

Figure 4.68: Time evolution of POD coefficients (black) and least-squares fit (blue).

main yields mode coefficients according to Equation (2.20), which are then combined to determine the time evolution of the low-order dynamical system presented in Sec- 
tion 2.7. In order to pursue the formulation above, the snapshots, or more specifically, the mode coefficients must be sufficiently resolved in time to make appropriate estimates of time derivatives. The time constraint makes acquisition of data through experimental methods especially difficult, although techniques including time-resolved or dual-time PIV have been successfully employed in the past (Perret et al., 2006a).

Figure 4.68 shows the time derivative of select POD mode coefficients as time series in black. The coefficients shown are those used to compose the LODS below; the first 14 of 2000 POD modes account for approximately $34.5 \%$ of the integrated turbulence kinetic energy in the domain. It is clear from the figure that the dynamics of each mode vary according to mode number; $d a_{i} / d t$ shows larger, low-frequency features when $i$ is low and much higher frequency oscillations with increasing mode number. The blue line in each subfigure is the result of the least-squares fit detailed in Equation (2.42), wherein the coefficients are interrelated by a system of parameters of increasing polynomial order up to cubic terms. Agreeing with previous applications of the technique (Perret et al., 2006b; Hamilton et al., submitted for publication May, 2016), the fit time derivatives of coefficients $d \hat{a}_{i} / d t$ (denoted with a caret) agree quite well when the mode number is low, and low-frequency dynamics are well characterized by the LODS. In the current application, the match between $d \hat{a}_{i} / d t$ and $d a_{i} / d t$ is nearly without error until the highest included mode, where the match shows reduced amplitudes and effects akin to low-pass filtering.

The fit of $d \hat{a}_{i} / d t$ requires that each of the modes interact with the others in the truncated POD basis, often at several polynomial orders. Figure 4.69 show the parameters coupling the dynamic evolution of mode coefficients for numerical solution according to equation (2.40). As expected, the linear term is dominant in magnitude. 


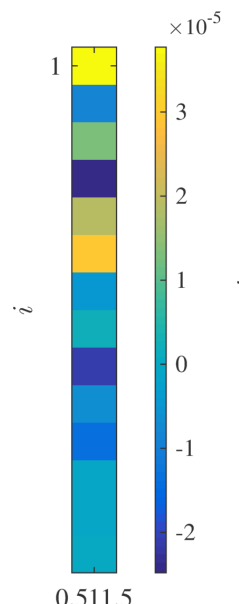

(A) Constant parameter

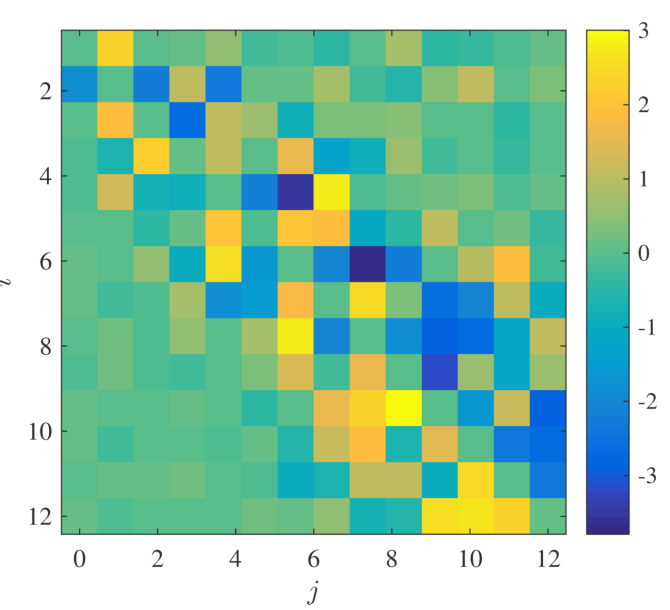

(B) Linear parameter

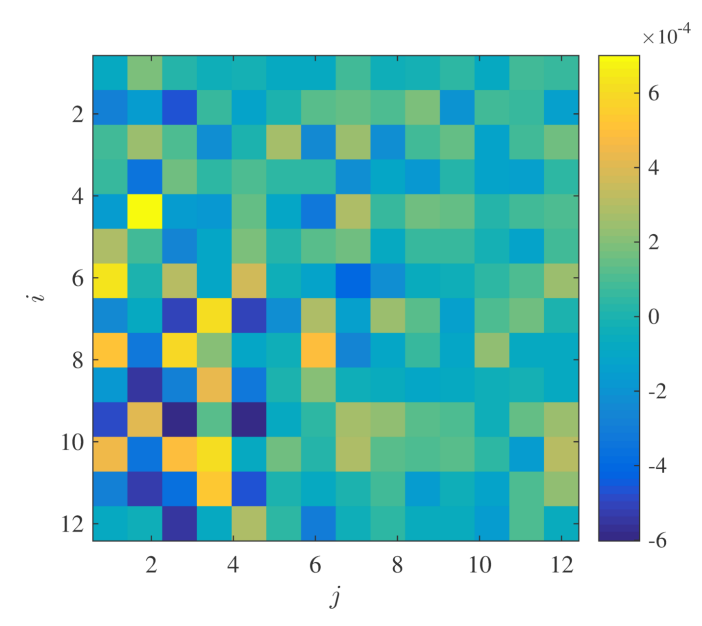

(C) Quadratic parameter with $k=1$

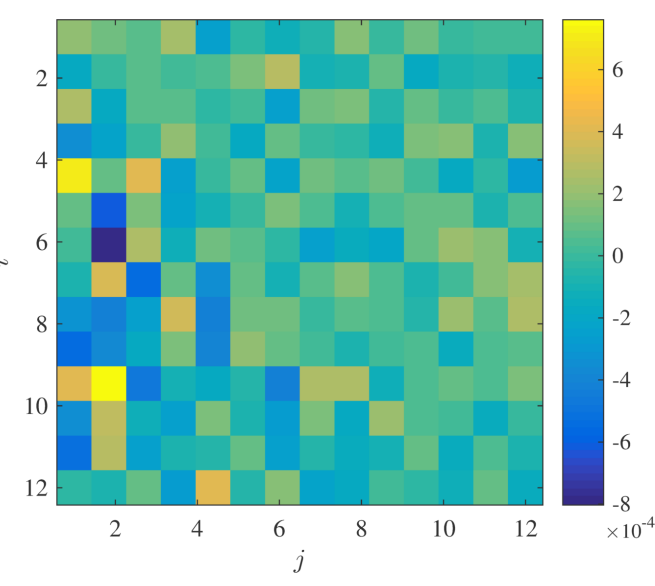

(D) Quadratic parameter with $k=2$

Figure 4.69: Constant 4.69(A), linear 4.69(B), and quadratic parameters $4.69(\mathrm{C})$ and $4.69(\mathrm{D})$ coupling the mode coefficients in the LODS.

The constant term is quite small $\left(\mathscr{O} \sim 10^{-5}\right)$ as each mode is zero-centered and shows no overarching trend in time. Quadratic terms relate the convection in the traditional Galerkin projection. Here, they are derived through a least-squares fit rather than projection onto the Navier-Stokes equations. They have small magnitudes $\left(\mathscr{O} \sim 10^{-4}\right)$, but dominate the constant terms and the cubic terms $\left(\mathscr{O} \sim 10^{-7}\right)$, not shown for brevity. 
Physical significance of the parameters is taken here to be analogous to triadic interactions discussed in wavenumber scale interaction in classical turbulence spectra. Similar parameters arise from mode projection in the Galerkin projection and are explored at length by Noack et al. (2003). There, the parameters relating dynamic evolution of the modes are classified in terms of their contributions to components of the momentum balance suggested in the Navier-Stokes equations, including inter-mode energy exchanges. While exploration of the parameters is beyond the scope of the current development, it is evident that each order of parameter shows behavior distinct from the others. The linear term in Figure 4.69(A) shows its most extreme values at low mode number. This is taken to indicate that low-frequency dynamics associated with the first few modes are most likely to need a correction centering the mean about zero, although they remain small in magnitude. The linear parameter $L_{i j}$ shown in Figure 4.69(B) shows trends where large magnitudes are near to, but off the diagonal. When the indices are equal $i=j$, magnitudes are identically 0 . Mode interactions appear to tend toward null values matching dissimilar indices (e.g. $i=1, j=12$ ). The linear parameter is nearly antisymmetric; for the near diagonal mode interactions, there is also a tendency for $L_{i j}>0$ for $i>j$ and vice versa. Behavior of the quadratic parameter is less easily diagnosed, although it appears that extreme values of $Q_{i j k}$ occur when $i>j$, in Figure $4.69(\mathrm{C})$. This trend is easily observed for low $k$ but becomes more difficult to asses as $k$ increases, as iFigure 4.69 (D).

The dynamic evolution of each mode coefficient is determined by solving the ODEs from equation (2.40). The behavior of each coefficient is coupled to the others through the LODS parameters $D_{i}, L_{i j}, Q_{i j k}$, and $C_{i j k l}$. Solving the system of equations results in the modeled coefficients shown as the blue time series in Figure 4.70. From the 

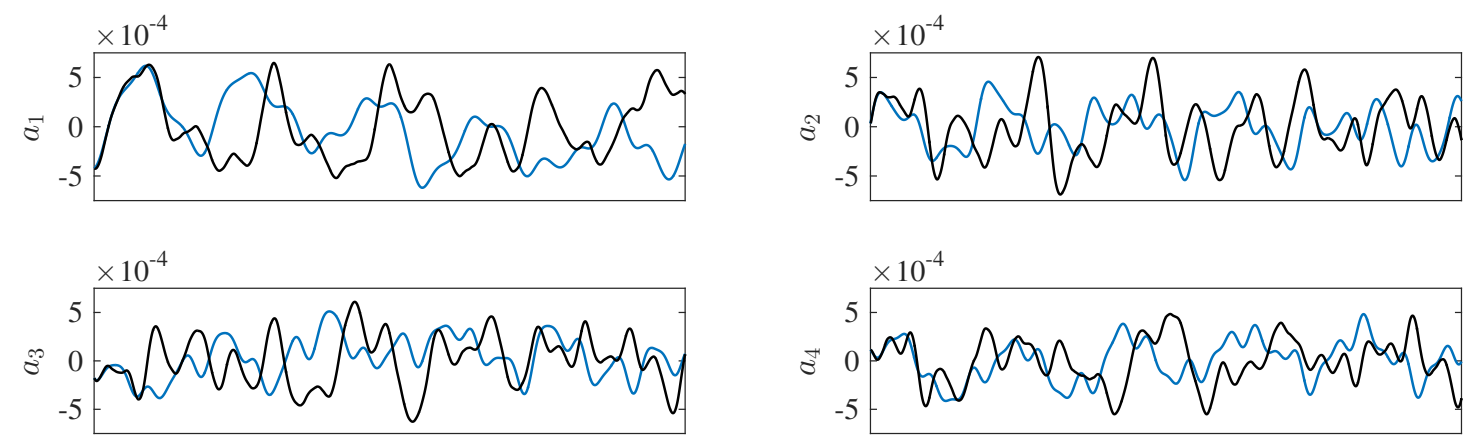

$\therefore 10$

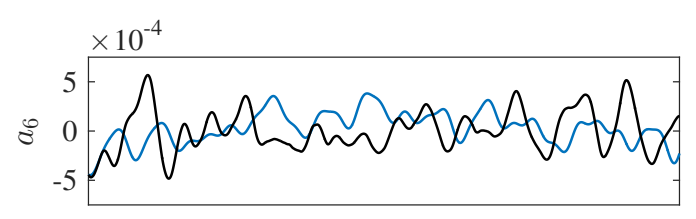

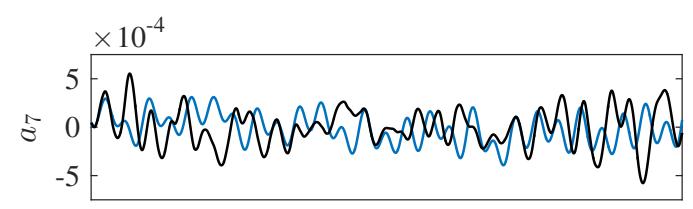
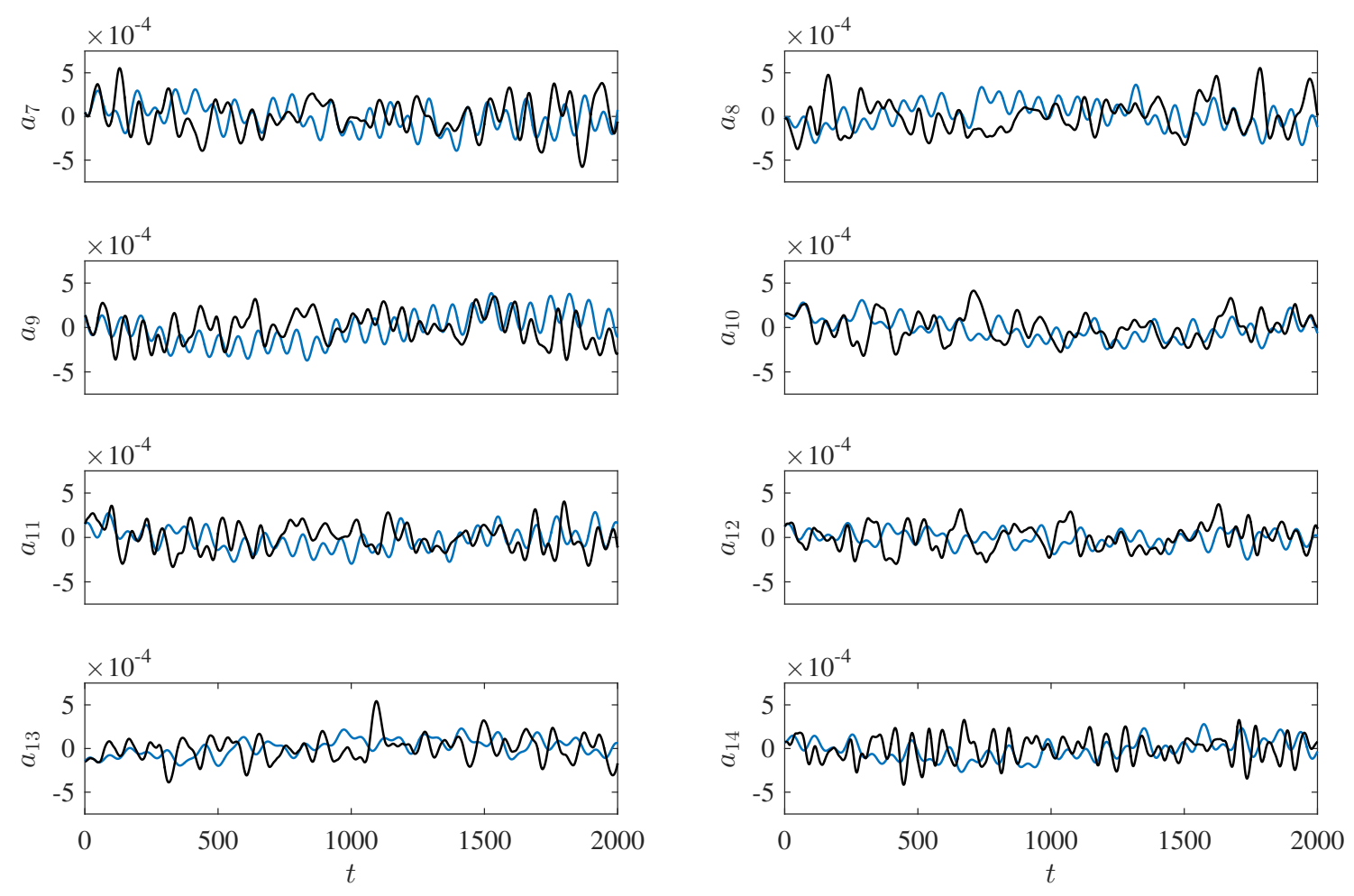

Figure 4.70: POD coefficients (black) and predictions from the coupled ODEs in the low-order dynamical system (blue).

figure it is clear that the estimated coefficients $\hat{a}_{i}$ diverge from their respective values produced through back-projecting the POD modes onto the snapshot basis, shown in 
black. The modeled coefficients are sought in a prescribed time interval, set here to match the span of the original snapshots, and require initial conditions, taken here as the first values of the POD coefficients $a_{i}(t=0)$. Modeled coefficients match quite well for a short range beyond their initial conditions but take on different trajectories as error accumulates from numerical solution of the ODEs and in the least-squares fits of their respective time derivatives.

While the trajectories of $\hat{a}_{i}$ are certainly different from $a_{i}$, Figure 4.70 demonstrates that each modeled coefficient exhibits dynamics of similar amplitude and frequency range as their respective match from the POD. Resolution in time of the modeled coefficients is determined by the error tolerances allowed in numerical integration of Equation (2.40). Including a greater number of modes in the LODS increases their ability to match the trajectories of $a_{i}$ but require significantly more computing power and time to resolve. Regardless of the number of modes used in the LODS, the system of $\hat{a}_{i}$ will eventually diverge and grow unstable given a sufficiently long prediction horizon.

Long time instability of the LODS is a detriment to making predictions of turbulent dynamics in the wind turbine wake. Additionally, the modeled mode coefficients take on trajectories that are sensitive to their initial conditions and may not reflect influence of the incoming atmospheric flow field. To keep the LODS coefficients from growing unstable, a means of recalibration is needed. Any means of recalibration or tuning of the dynamical system must respect incoming dynamics if the low-order model is to be used in a predictive sense. To these ends, a series of transfer functions that relate an input signal to the modeled coefficients is sought. The input signal should characterize the flow encountered by the wind turbine rotor if proposed model is to reflect the physical conditions experienced by a wind turbine. 
For the purposes of informing the dynamical system, the input signal is taken as the effective velocity seen by the rotor disk, defined by Equation 3.3. The rotor area is shown in the schematic depicting the simulation domain as a light gray circle circumscribing the swept area of the rotor. The input signal is used to periodically predict refreshed initial conditions for the LODS of the wind turbine wake, shown in the flowchart in Figure 4.71. Process blocks are shown as diamonds and include decomposition into modes, tuning initial conditions from transfer functions, calibration of the low-order dynamical system, and reconstruction of the turbulence field in the wake. Inputs are shown as rectangles and include as statistical record of the wake, taken here as the LES data described above, the effective inflow velocity used as the 'upstream sensor,' and the output is the modeled wake flow $\hat{u}_{\text {wake }}$ Intermediate variables are shown in squares with rounded corners and include the POD modes $\left(\phi^{(i)}\right)$ and coefficients $\left(a_{i}\right)$, the renewed initial conditions $\left(a_{i, 0}^{\prime}\right)$, as well as the modeled coefficients $\left(\hat{a}_{i}\right)$

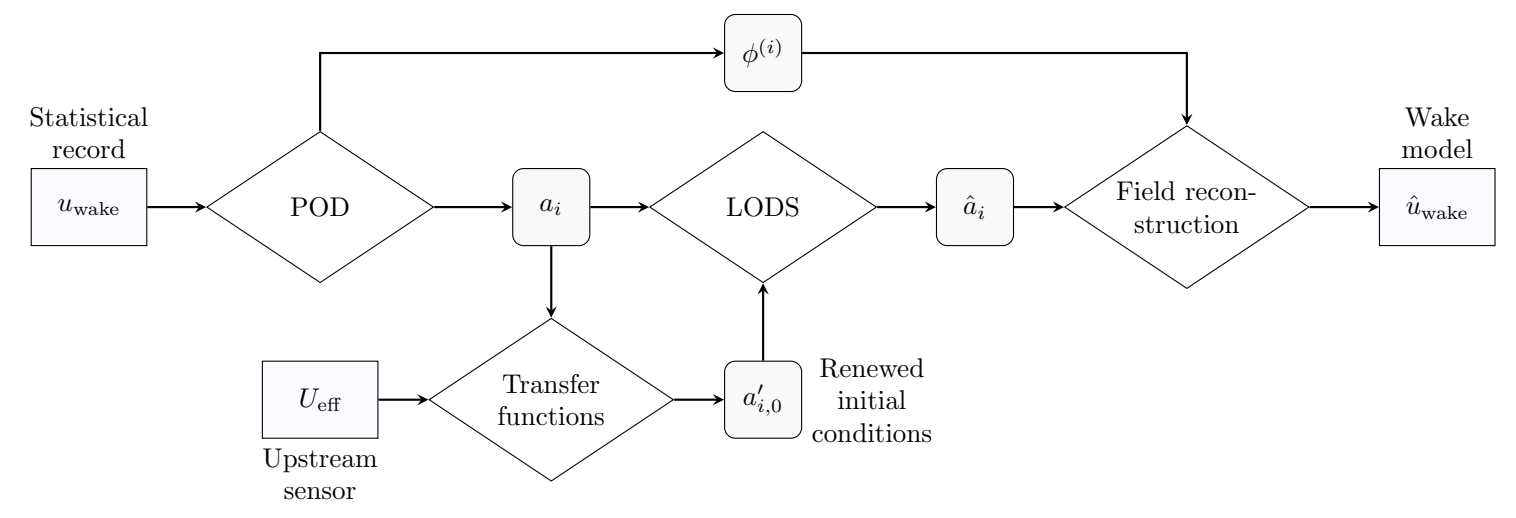

Figure 4.71: Work flow demonstrating the input/output map of the dynamical system with periodic recalibration from the effective inflow velocity.

Figure 4.72 shows $U_{\text {eff }}(t)$ and is separated into two ranges. The portion of the signal shown in blue indicates the range over which $U_{\text {eff }}$ was compared to $a_{i}$ to define trans- 


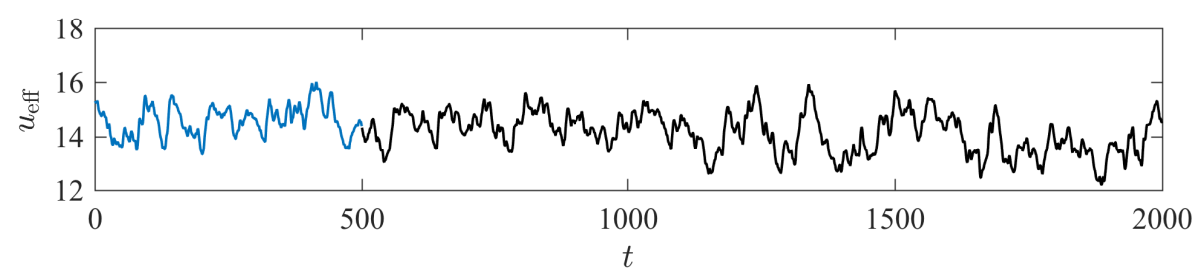

Figure 4.72: Effective incoming velocity. Time used to define transfer functions shown in blue. Validation time shown in black.

fer functions. The portion of the signal shown in black is used as a validation range below. This signal $U_{\text {eff }}$ is commonly used in the calculation of the fluid power available to the turbine. For calculation of the transfer functions, $U_{\text {eff }}$ is normalized to vary over the interval $[0,1]$. Normalization maintains the variation of the signal that characterizes the incoming turbulence and correlates with fluctuations in power production by the device. The effective inflow velocity signal is compared to POD mode coefficients over the same time interval that have been similarly normalized. Transfer functions are calculated relating the inflow velocity signal to the POD coefficients in the wake optimizing the number and magnitudes of poles and zeros for best fit between $U_{\text {eff }}$ and $a_{i}$

Open loop transfer functions are employed to estimate new initial conditions for the dynamical system of the wake corresponding to the atmospheric flow interacting with the wind turbine rotor. Optimized transfer functions exhibit as many as 10 poles and 9 zero locations, and the fit ranges between 40 and $80 \%$. Table 4.4 shows details of the transfer functions employed in the recalibration process.

Periodically refreshing the system with new initial conditions forces the solution to reflect the influence of incoming velocity within the error bounds of the transfer functions and dynamical system. However, once recalibrated, the dynamical model is again free to take on its own trajectory that may deviate from that of the physical 
Table 4.4: Number of poles, zeros, and the goodness of fit for transfer functions relating $U_{\text {eff }}$ to each mode coefficient.

\begin{tabular}{c|rrrrrrr} 
Mode & 1 & 2 & 3 & 4 & 5 & 6 & 7 \\
Poles & 7 & 10 & 10 & 8 & 10 & 10 & 10 \\
Zeros & 2 & 5 & 4 & 7 & 8 & 9 & 8 \\
Fit (\%) & 78.37 & 81.43 & 65.18 & 64.24 & 63.48 & 71.53 & 56.70 \\
& & & & & & & \\
Mode & 8 & 9 & 10 & 11 & 12 & 13 & 14 \\
Poles & 10 & 9 & 9 & 10 & 10 & 9 & 10 \\
Zeros & 6 & 7 & 8 & 7 & 7 & 8 & 9 \\
Fit (\%) & 60.16 & 50.42 & 54.90 & 50.37 & 54.83 & 50.67 & 41.42
\end{tabular}

system. Thus the degree of fit between the model and the true coefficient space is necessarily a function of the time interval between periodical recalibrations. Large time intervals between the resupply of fresh initial conditions allows the modeled system to deviate further from the validation data, taken as the POD coefficients. Figure 4.73 compares several coefficients predicted by the dynamical model $\hat{a}_{i}$ to their respective validation data for a range of prediction periods. Error is dramatically reduced in the resulting system by shortening the period over which new estimates of the initial conditions are generated. Longer periods between recalibration (top of each subfigure) allow $\hat{a}_{i}$ to deviate far enough from $a_{i}$ that the transfer function is unable to find correct initial conditions.

The goodness of fit between $\hat{a}_{i}$ and $a_{i}$ is calculated using the normalized rootmean-square deviation between the two signals,

$$
\text { fit }(\%)=\left(1-\frac{\left\|a_{i}(t)-\hat{a}_{i}(t)\right\|}{\left\|a_{i}(t)-\bar{a}_{i}\right\|}\right) \times 100
$$

where the $\|\cdot\|$ operator indicates the 2 -norm included argument and the overline $\bar{a}_{i}$ 

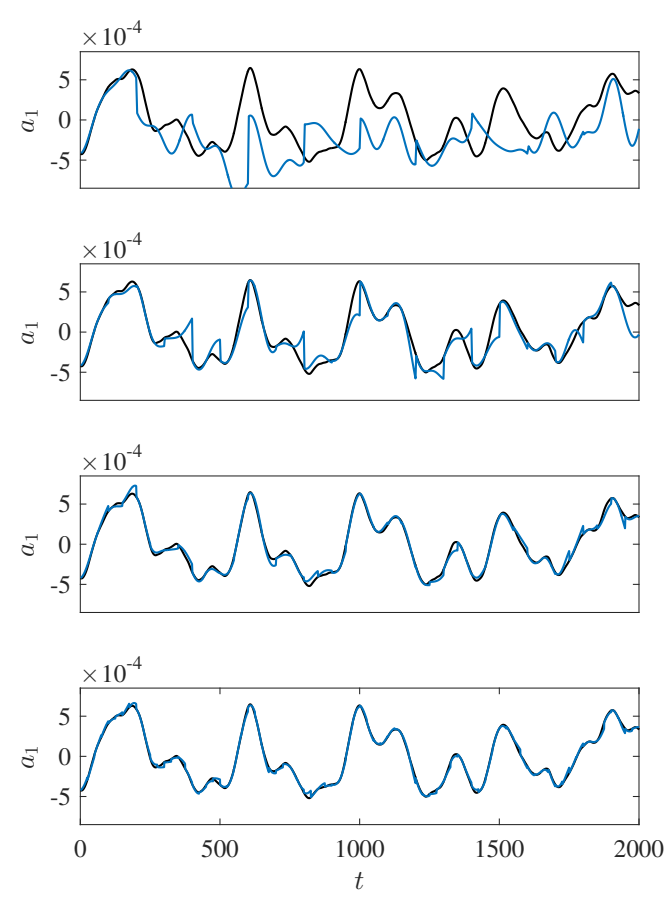

(A) Coefficient prediction for mode 1 .
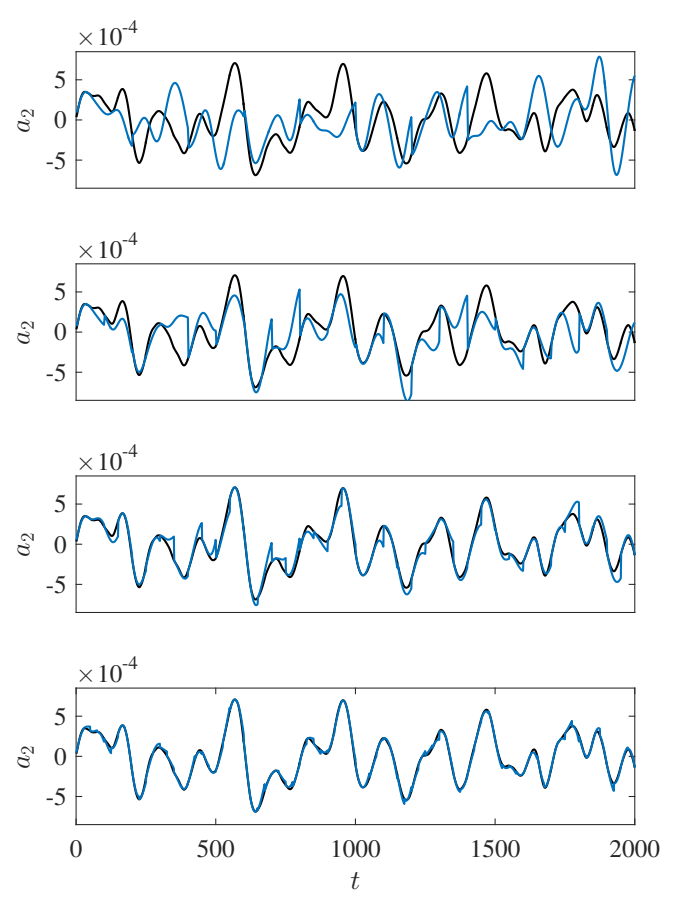

(B) Coefficient prediction for mode 2 .

Figure 4.73: Comparison of LODS coefficient predictions with recalibration at a variety of periods. In each subfigure from top, $T_{p} \in[200,100,50,25]$ seconds.

implies the ensemble average of each coefficient. A range of time intervals were tested for each mode coefficient, shown in Figure 4.74. The trends in the figures suggest that as the period between renewed initial conditions $T_{p}$ is reduced, the fit tends toward $100 \%$. On the contrary, large time periods between recalibration allow the system to deviate far enough that the transfer functions are not able to supply the appropriate corrections. In the case where $T_{p}=200$ seconds, the fit is negative, implying that the modeled coefficients fall out of phase with their POD equivalents.

As recommended by the fit analysis, a short time period between recalibration is selected for the model. Figure 4.75 compares the modeled coefficients to the validation data for the range of modes used in the LODS. When contrasted against the trends 


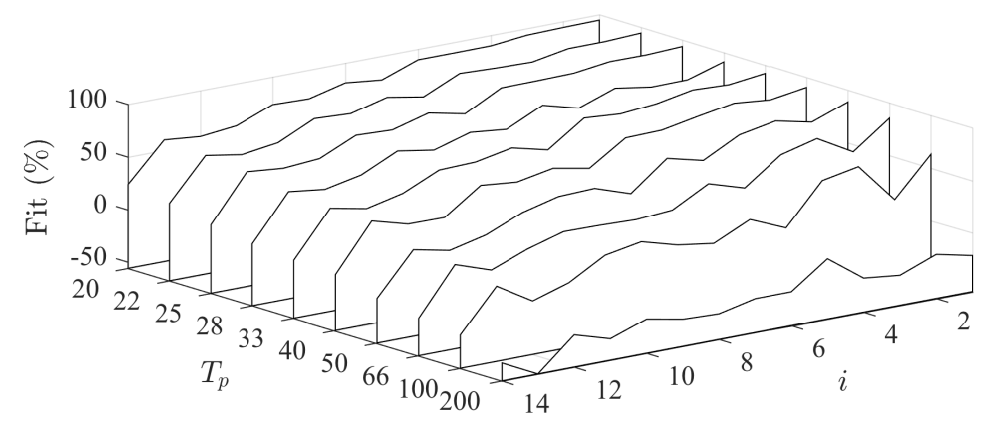

Figure 4.74: Fit between coefficients predicted by dynamical model and their respective values from POD. Coefficients for each mode $i$ are tested for a range of time periods $T_{p}$ (in seconds).

seen in Figure 4.70, transfer functions indeed supply the necessary information to the LODS to inform the model of the inflow. Modeled coefficients $\hat{a}_{i}$ exhibit discontinuities that arise from the abrupt recalibration of the dynamical system. Discontinuities are difficult to detect for low mode numbers but become more obvious at higher $i$. With modeled mode coefficients, fluctuating velocity snapshot for the wake are calculated by superposition of the POD modes and the LODS output coefficients as,

$$
\hat{\boldsymbol{u}}_{\text {wake }}=\sum_{i=1}^{N_{r}} \hat{a}_{i} \Phi^{(i)},
$$

where $N_{r}$ is the number of modes used in the reduced order model. Shocks seen in modeled mode coefficients propagate through to fluctuating velocity snapshots in the wake as abrupt shifts in the velocity fields in time. The time series of velocity snapshots output by LODS for the wake by Equation 4.21 are compared in Figure 4.76. Trends show the filtering effects of the POD in time. Velocity signals produced by the LODS (blue) exhibit strong low-pass filtering as compared to the LES data, taken as validation. Additionally, discontinuities present in the predicted coefficients are seen in the velocity signals as well. Low-pass filtering is mitigated by adjusting the point of trun- 

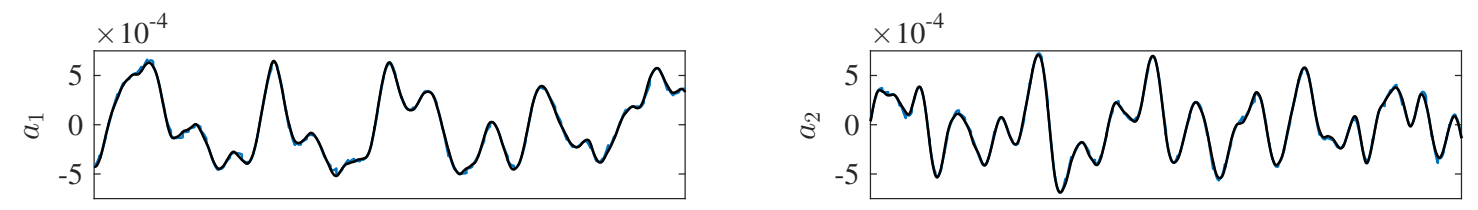

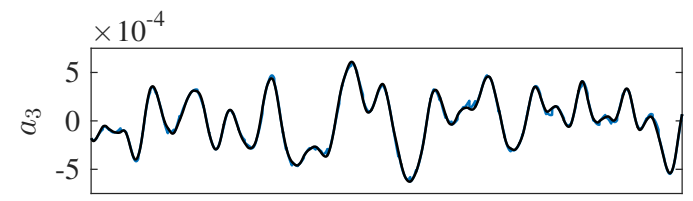

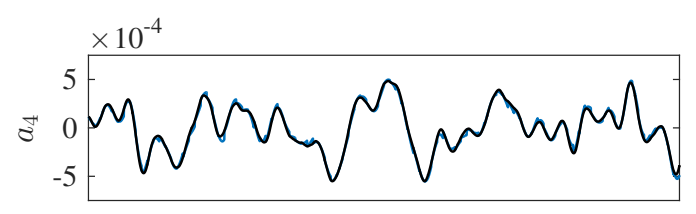

0

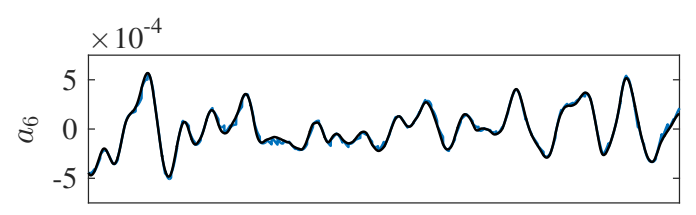

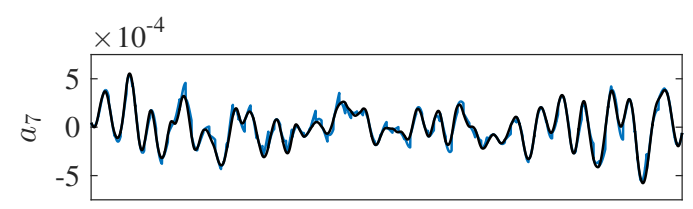
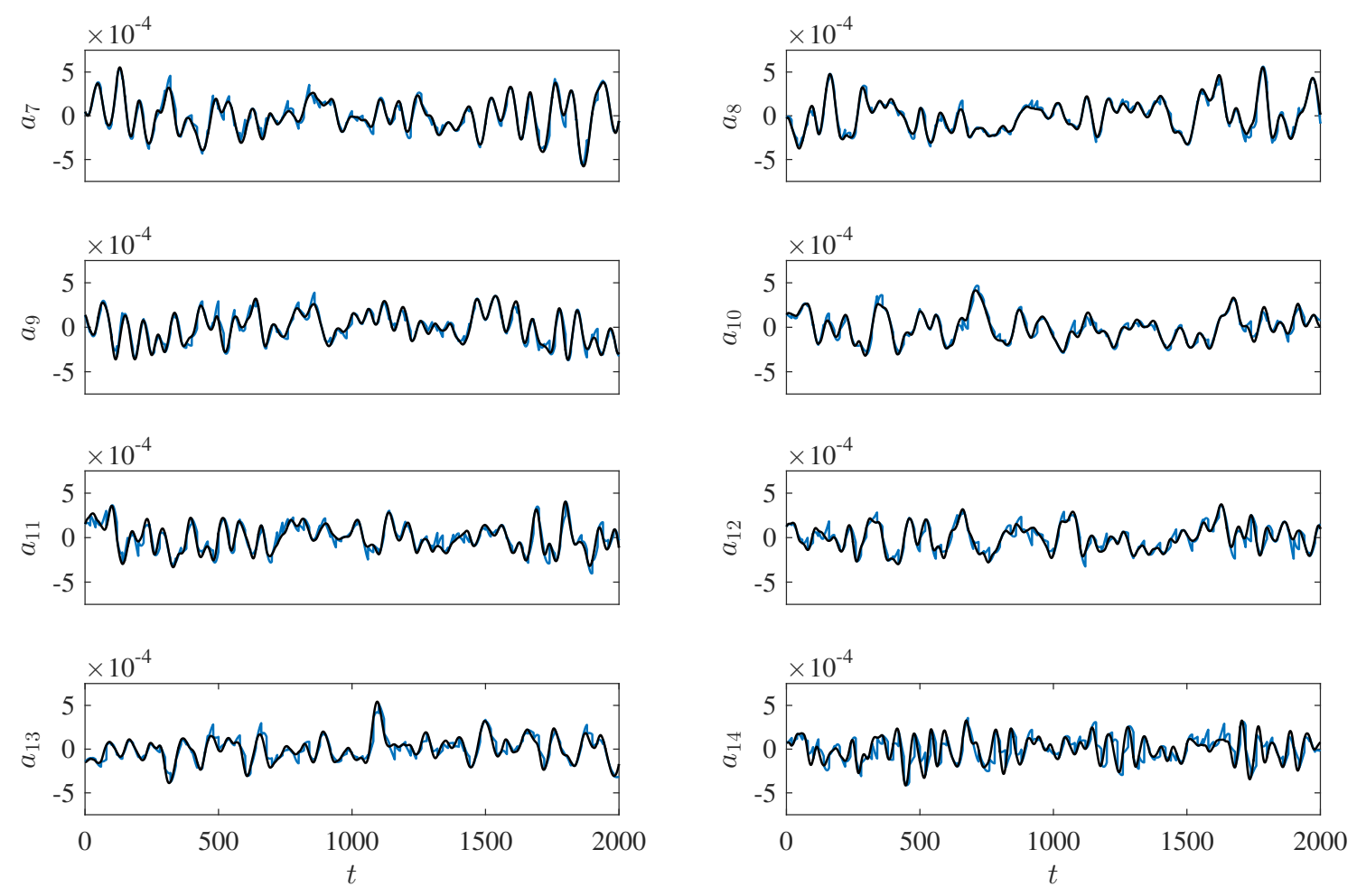

Figure 4.75: LODS coefficient predictions periodically recalibrated with initial conditions via effective inflow velocity and transfer functions.

cation of the POD mode basis accounting for both spatial filtering through the modes $\left(\phi^{(i)}\right)$ and temporal filtering through the associated coefficients $\left(a_{i}\right)$. For comparison, 
Figure 4.76 shows the time series predicted by the POD in green. The nearly complete overlap of the LODS and the POD coefficients confirms that the dynamical system introduces very little filtering of the predicted velocity fields beyond that of the POD.
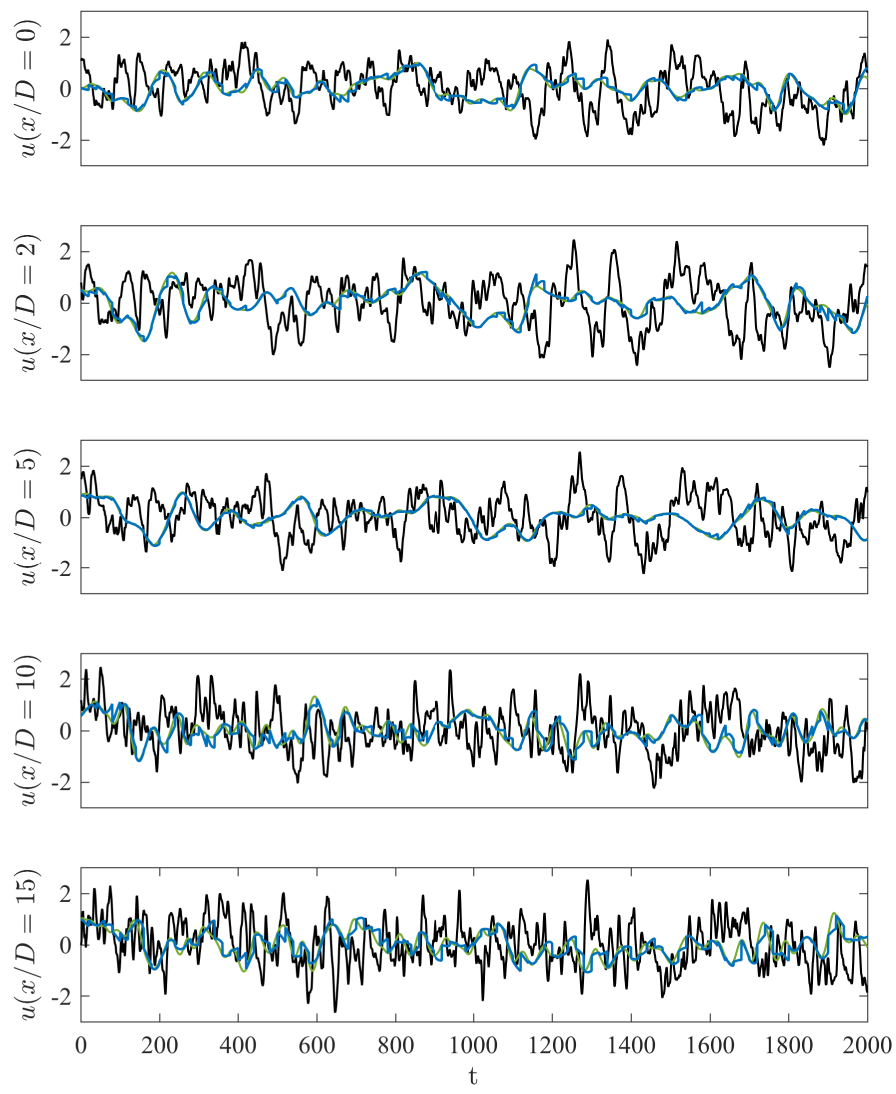

Figure 4.76: Comparison of velocity signals at locations downstream of the wind turbine. Blue lines are $\hat{u}_{\text {wake }}$ integrated over the swept area of the rotor, black lines are validation data from the LES, green lines are produced with POD.

In ensemble, discontinuities are washed out and the turbulence statistics in the modeled wake more closesly reflect expected features. Terms from the modeled turbulent stress tensor are shown in Figures 4.77 and 4.78. The color scale of each subfigure is fixed to that of the original statistical values of each stress for ease of comparison.

The modeled streamwise normal stress $\hat{u u}$ demonstrates the same characteristic 


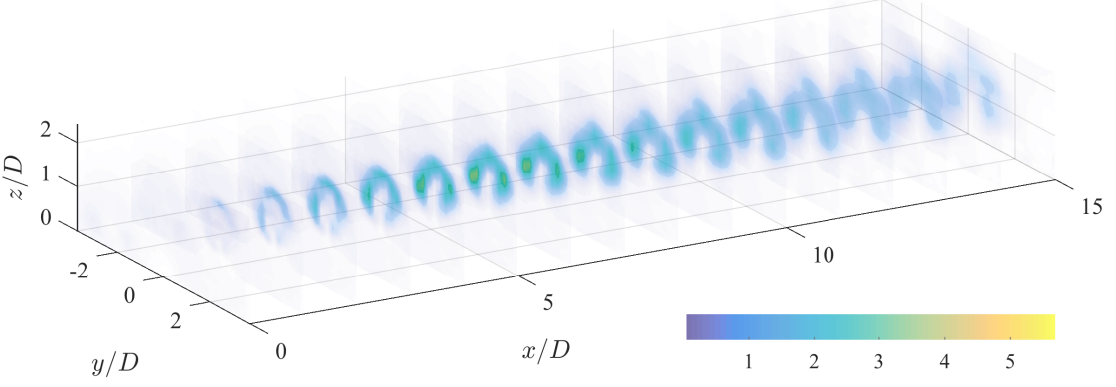

(A) $\hat{u u}$

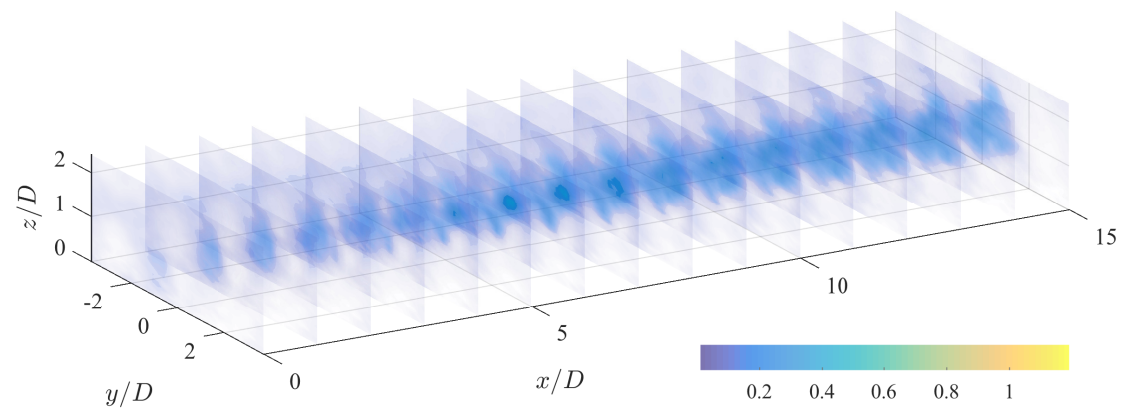

(B) $\hat{v v}$

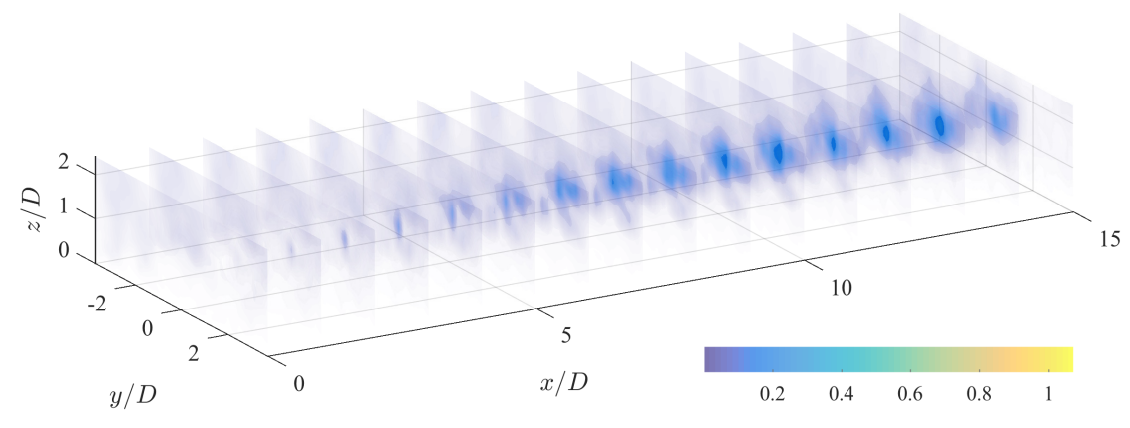

(C) $\hat{w w}$

Figure 4.77: Wake model Reynolds normal stresses

feature evolving with $x / D$ trailing the top tip of the rotor disk. It is expected that the modeled stresses will demonstrate reduced magnitudes compared to those arising from the original statistics. The difference in magnitudes is attributed to truncating the basis of modes to $N_{r}$ in the model and reconstruction. Here only 14 of 2000 modes are used and account for $34.5 \%$ of the total turbulence kinetic energy of the wake. Ex- 


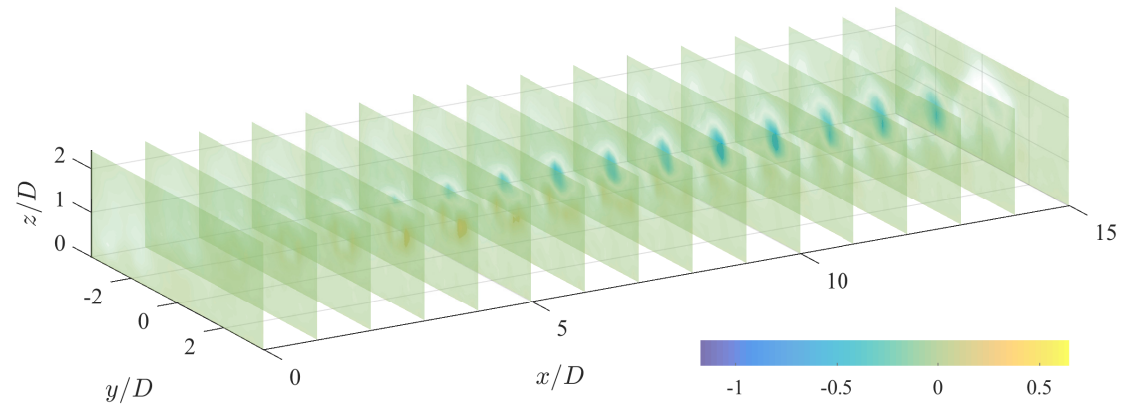

(A) $\hat{u v}$

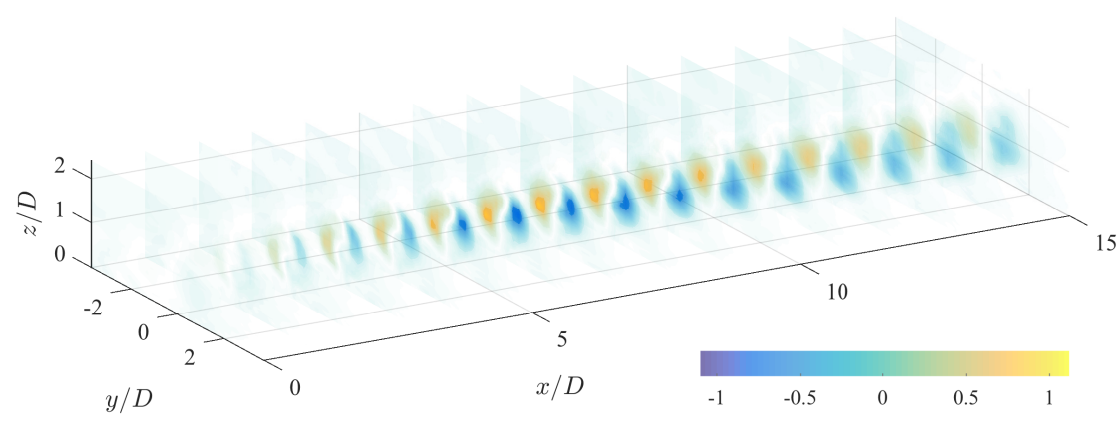

(B) $\hat{u w}$

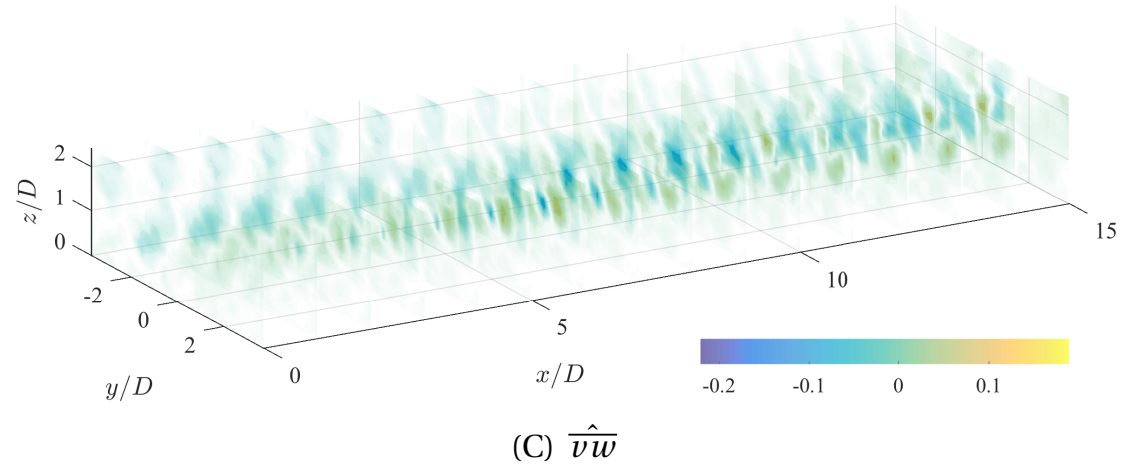

Figure 4.78: Wake model Reynolds shear stresses

pected structures in the spanwise and wall-normal Reynolds normal stresses are also well represented with the LODS, seen in Figures 4.77(B) and 4.77(C). The structures are shown in blue rather than yellow as was the case above due to the exclusion of energy from the model.

Shear terms from the modeled Reynolds stress tensor are easier to see than the nor- 
mal components. It is well established that the shear terms are more easily represented by the POD, as the dynamics exhibit smaller magnitudes and the quantities are more or less symmetrically distributed about zero. Figure 4.78 indicates that the shear terms $\hat{u v}$ and $\hat{v w}$ are nearly identical to their original statistical values. The same trend is true for the streamwise/wall-normal stress, although it is moe difficult to visualize as the distribution of $\hat{u v}$ is skewed toward negative values.

Previous exploration of error propagation through the POD (Hamilton et al., (submitted for publication March, 2016) demonstrated that much of the energy excluded in the basis truncation can safely be considered as homogeneously distributed isotropic turbulence. It was there established that a tensor of constant coefficients could be defined to correct the magnitudes of each stress to account for energy excluded in the reduced order descriptions of the flow. Correction arises from the observation that isotropic contributions to the full turbulence fields are both small and fairly homogeneous, indicating that the energy excluded from the flow using $\frac{\circ}{u_{i} u_{j}}$ may be considered as nearly constant background energy. Recent extensions of the double POD (Hamilton et al., 2016) corrected estimates of the Reynolds stresses by way of a constant coefficients used to push the magnitudes of each component toward values seen in the full statistics,

$$
\overline{u_{i} u_{j}}=C_{i j} \hat{u_{i} u_{j}}
$$

The correction coefficient $C_{i j}$ is found through a minimization of the root-meansquare error between the statistical stress field and the corrected reduced order model. Here, effects of model correction are shown by way of the goodness of fit calculation from Equation (4.20). The fit of the model before and after correction with $C_{i j}$ are listed in Table 4.5, showing increases in the goodness of fit of the model up to $36 \%$. 
Table 4.5: Comparison of goodness of fit values with and without application of constant correction.

\begin{tabular}{cccc} 
Reynolds stress & Raw fit (\%) & Correction factor & Corrected fit (\%) \\
\hline$\hat{u} u$ & 22.49 & 2.20 & 58.29 \\
$\hat{v v}$ & 64.49 & 4.04 & 81.70 \\
$\hat{w w}$ & 75.12 & 6.92 & 89.11 \\
$\hat{\overrightarrow{u v}}$ & 90.75 & 1.57 & 93.69 \\
$\hat{u w}$ & 85.23 & 2.60 & 91.21 \\
$\hat{v w}$ & 97.16 & 1.18 & 97.18
\end{tabular}

Correction coefficients reported in the table differ slightly from previously reported values in Hamilton et al. (2016). Differences seen in $C_{i j}$ here arise from a variety of causes; most significantly, the data analyzed here issues from the large eddy simulation of a single wind turbine in isolation rather than experimental data of the wake of a wind turbine in a fully-developed array, where dynamics are expected to be significantly different. The spatial range of the LES data taken as input for the LODS is also significantly larger here than in previous experimental work. Correction coefficients and gains in goodness of fit presented in Table 4.5 are similar in magnitude and distribution to previous work. Correction coefficients are expected to vary depending on the specific data in question. As explored in Section 4.3, correction coefficients also decrease nonlinearly as functions of the point of truncation of the POD basis.

\subsubsection{Conclusions}

Predictive models of wind turbine wakes are necessary for optimal design and operation of large wind turbine arrays, although the complex turbulent wake flows remain a challenge. Large eddy simulations such as the one analyzed herein provide increas- 
ingly real physics with the necessary temporal and spatial resolution to determine loading conditions and dynamic evolution of the wake. However, the computational cost of LES prohibits the method from being applicable for continuous-time modeling or monitoring of wake flows. In many wind farms, monitoring of wind turbine wakes and operating conditions is difficult, if not prohibitively expensive. Modeling and prediction as in the scheme ad undertaken here present a possible path forward for diagnosing control schemes and monitoring techniques easily and uniquely adapted to wind turbines in the field.

Using high resolution data from LES simulations, the dominant features of the turbulence flow field are characterized using the proper orthogonal decomposition, optimal for describing the energy of the flow with the fewest modes. Low-dimensional models are generated combining the time series of mode coefficients through a leastsquares error minimization of their time derivatives. The dynamic evolution of the mode coefficients are coupled into ordinary differential equations by way of polynomial coefficients up to cubic order. Solving the coupled set of ODEs results in predictions of mode coefficients that can then be used to reconstruct fluctuating velocity fields in the wind turbine wake. Predicted trajectories of the mode coefficients are sensitive to initial conditions, diverging from validation trajectories and ultimately becoming unstable. Residual error of the least-squares fit defining the coupling parameters of the LODS propagate through to the ODEs and accumulate in their solution over time.

The dynamical wake model is trained to reflect the influence of atmospheric flow incident to the wind turbine rotor. A control scheme is employed using open-loop transfer functions that periodically supply refreshed initial conditions to the system, 
characterizing the effective inflow velocity. Validation data of the mode coefficients are compared to predicted values employing recalibration a variety of time intervals, showing that a nearly perfect fit is possible with sufficiently short prediction horizons. Transfer functions are optimized for their ability to match the input time series of the POD coefficients, constrained only by a maximum of 10 poles. A comprehensive description of the transfer functions, their estimation, and behavior is provided in E. The recalibration method applied here is equally feasible for any dynamical system, provided an input signal is readily available to inform the time evolution of the mode coefficients.

Fluctuating velocity snapshots are reconstructed through superposition of the products of the truncated POD mode basis and the respective coefficients predicted by the low-order dynamical system tuned to reflect the incoming flow. Time series of velocities in the wake show effects of low-pass filtering resulting from truncation of the POD basis. Discontinuities in the mode coefficients result from periodically halting the model and applying new initial conditions. Abrupt changes in the mode coefficients propagate though to velocity signals, although their influence is washed out in ensemble averaged wake statistics. Predictions of the Reynolds stress tensor in the wind turbine wake are corrected following the method put forth in Hamilton et al. (2016), yielding improvements of estimated wake statistics up to $36 \%$. 


\subsection{Reduced-order wind turbine array model}

The open-loop transfer function control scheme pursued above is intended ultimately to act as a means of connecting wake models to one another in series. In this way, a wind turbine array may be modeled given a sufficiently robust statistical record and minimal continuous-time monitoring. As for an isolated wind turbine, a wake in an array is the object of focus for the reduced order model pursued here. Data for the model generation comes from a large eddy simulation with parameters closely tuned to match the simulation above. This ensures that results may be compared directly in future analyses. The relative placement of wind turbines in the simulation space are shown in the schematic in Figure 4.79.

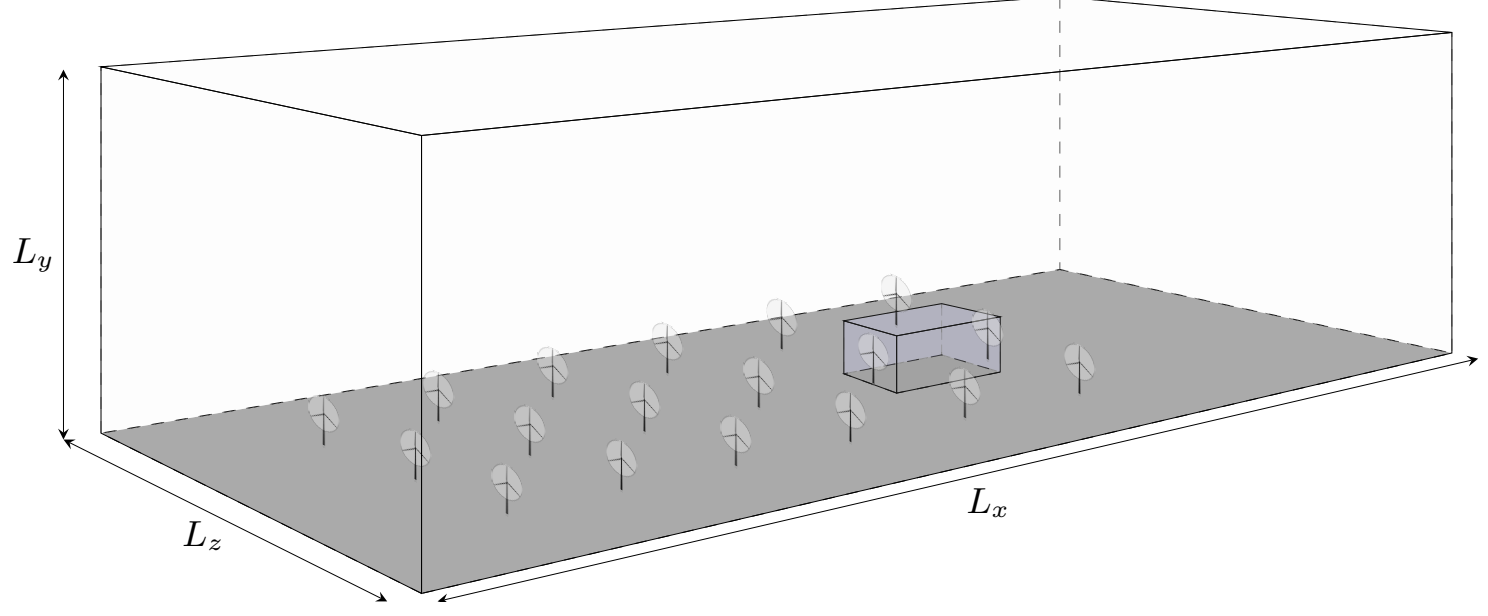

Figure 4.79: Schematic of wind turbine array LES indicating the area of focus for reduced order modeling.

In developing a model for a series of wakes, one can consider that each wind turbine in the simulation has a characteristic space, according to the spacing of devices in the array. Wake data analyzed for the model generation purposes is extracted from the full simulation space and may be considered as discrete and periodic wake elements 
that together account for the flow physics of the array. A detail view of a typical wake block extracted from the simulation is shown in Figure 4.80. Note that several such wake blocks are discussed in the current results. To distinguish easily between them, a subscript numeral IV will be used to identify results pertaining to the wake of a fourthrow wind turbine. Analogously, a subscript numeral V will be used to identify results pertaining to the wake of a fifth-row wind turbine.

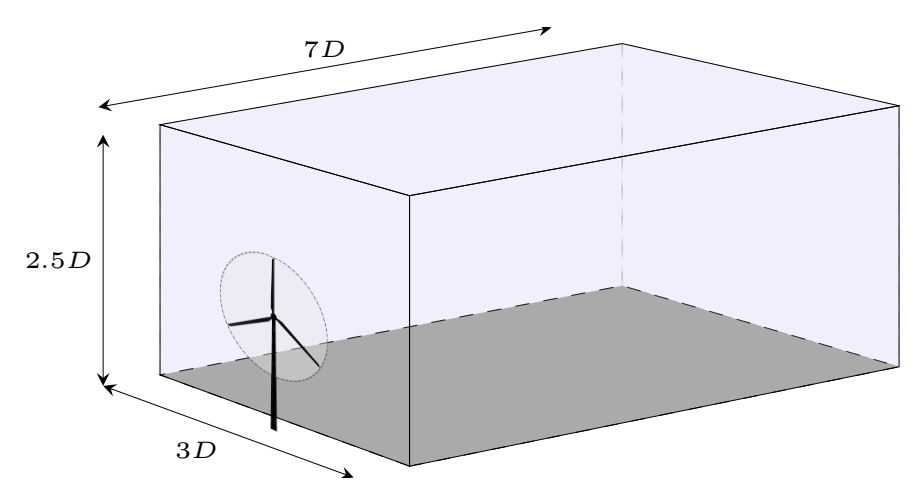

Figure 4.80: Detail view of a single wake block within the simulated array. Effective inflow velocity is to be calculated with the transfer-function calibrated LODS.

For brevity, statistics are shown only for wake IV. Statistical fields offered in Figure 4.81 serve as a comparison against the isolated wake above. Note that the streamwise coordinate is limited now to 8 rotor diameters according to the spacing in the simulated WTA. Cursory inspection shows similar qualities of each flow statistic in the figure, although they deviate quantitatively. The momentum deficit is shown as a blue region in Figure 4.81(A). The characteristic forms of wind turbine wake turbulence are shown in Figures 4.81(B) and 4.81(C), following the rotor area.

Results from the POD are not shown for wakes in the LES array. Eigenvalues of the decomposition show faster convergence of energy as compared to the isolated wake; eigenvalues associated with high mode numbers have moderate values, $\sim 10^{-6}$ rather 


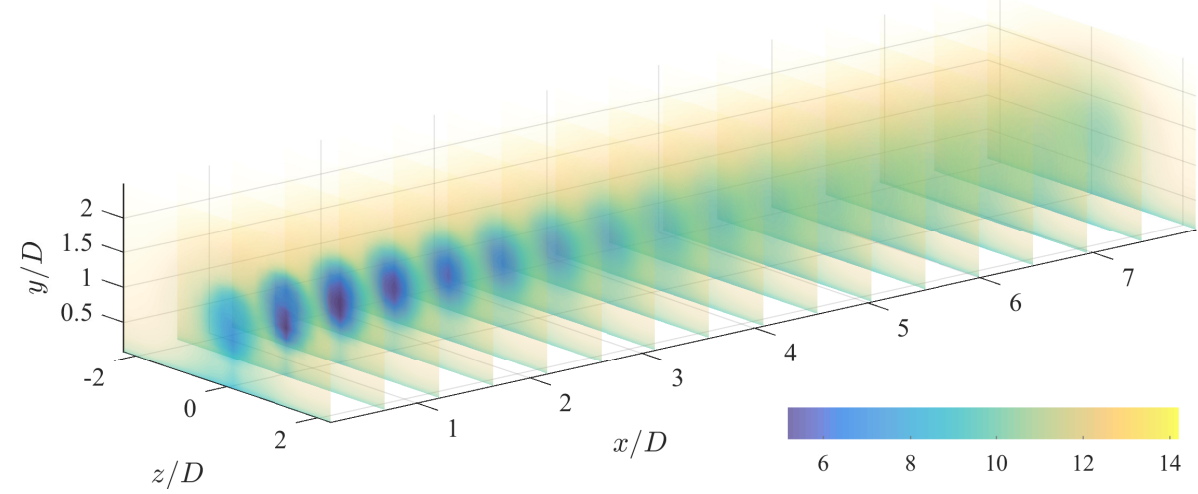

(A) $U_{\text {IV }}$

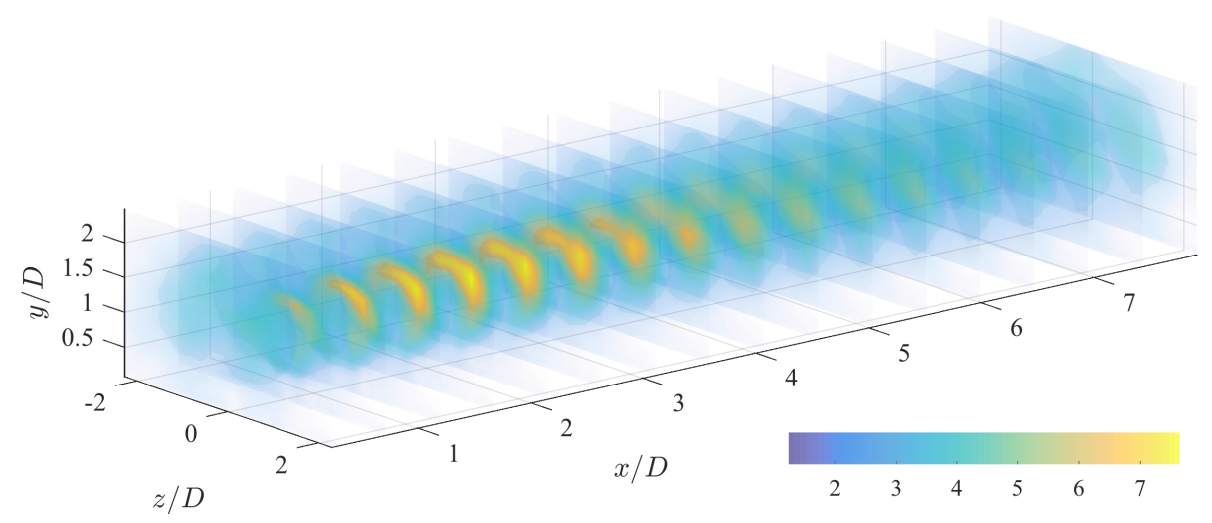

(B) $\overline{u u}_{\mathrm{IV}}$

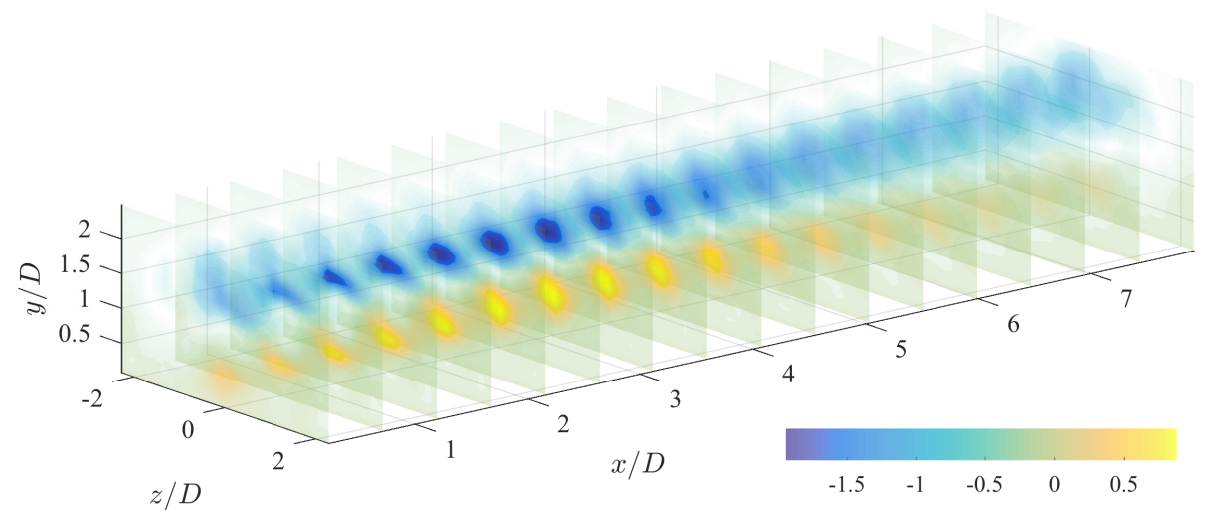

(C) $\overline{u v}_{\mathrm{IV}}$

Figure 4.81: Mean velocity (A), streamwise normal stress (B), and primary shear stress (C) in wake IV. 
than $\sim 10^{-9}$. The POD modes themselves are less like Fourier modes than those of an isolated wake, due to the inclusion of additional turbine rotors and a smaller interrogation area. Low-order dynamical systems are identified in the same manner as for both the channel flow DNS and for the isolated wind turbine wake LES above. Parameters coupling the mode coefficients are shown in Figure 4.82 for wake IV. The basis was truncated to 12 modes in wake IV, accounting for approximately $46 \%$ of the turbulence kinetic energy.

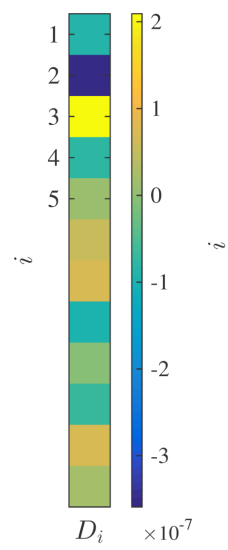

(A) $D_{i, I V}$

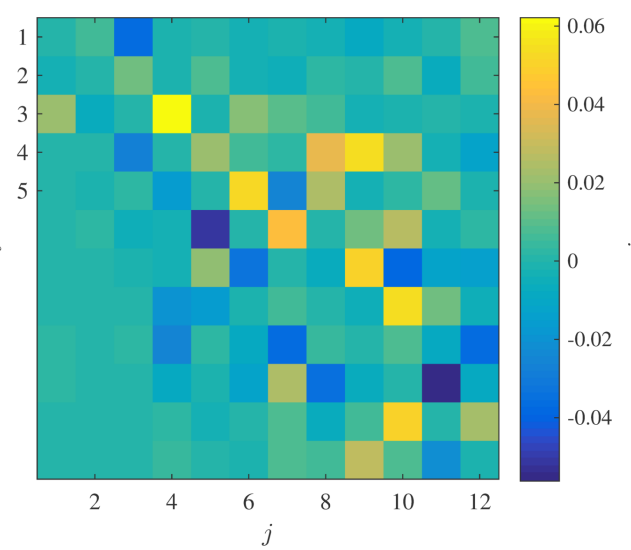

(B) $L_{i j, I V}$

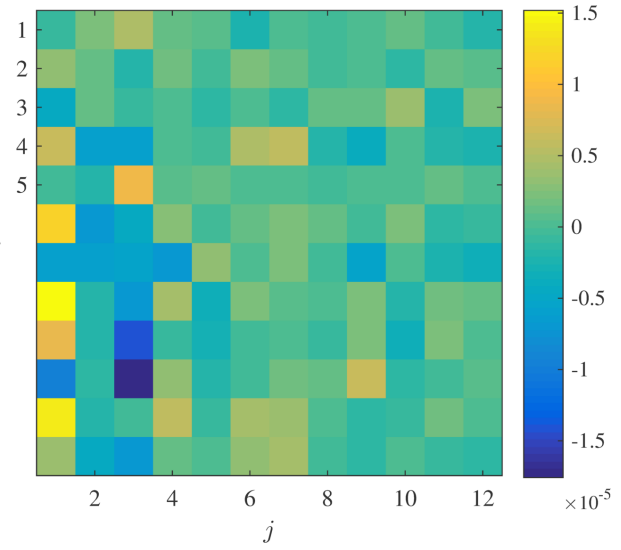

(C) $\mathrm{s} Q_{i j 1, I V}$

Figure 4.82: Parameters coupling dynamic modes of wake IV.

Results showing the time derivatives of the POD coefficients and their respective least-squares fits have been omitted from the current manuscript. Solving the system of 12 coupled ordinary differential equations results in the LODS predictions of mode coefficients $\hat{a}_{i}$, shown in Figure 4.85. The modal basis employed in the LODS here accounts for a greater portion of the relative kinetic energy than that of the isolated wind turbine, although it does so with fewer modes. Additionally, the turbulence intensity is much higher in the wake within a large array than in isolation. The resulting dynamical system shows greater disparity between calculated and modeled coefficients. 

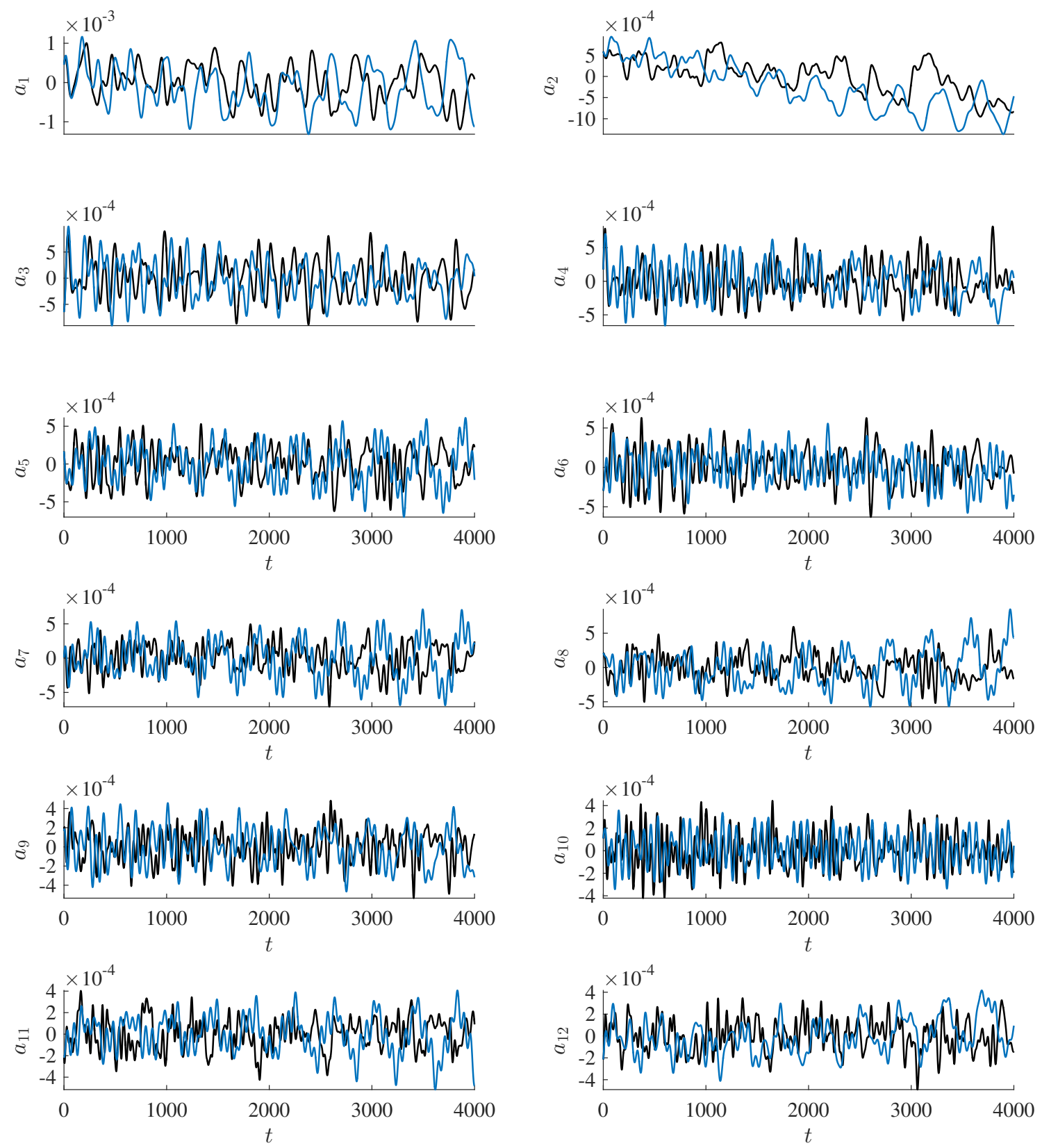

Figure 4.83: POD (black) and LODS (blue) coefficients corresponding for the 12mode system in wake IV.

Open-loop transfer functions are formulated as above, scanning a range of poles and zeros to maximize the goodness of fit between calculated mode coefficients and 

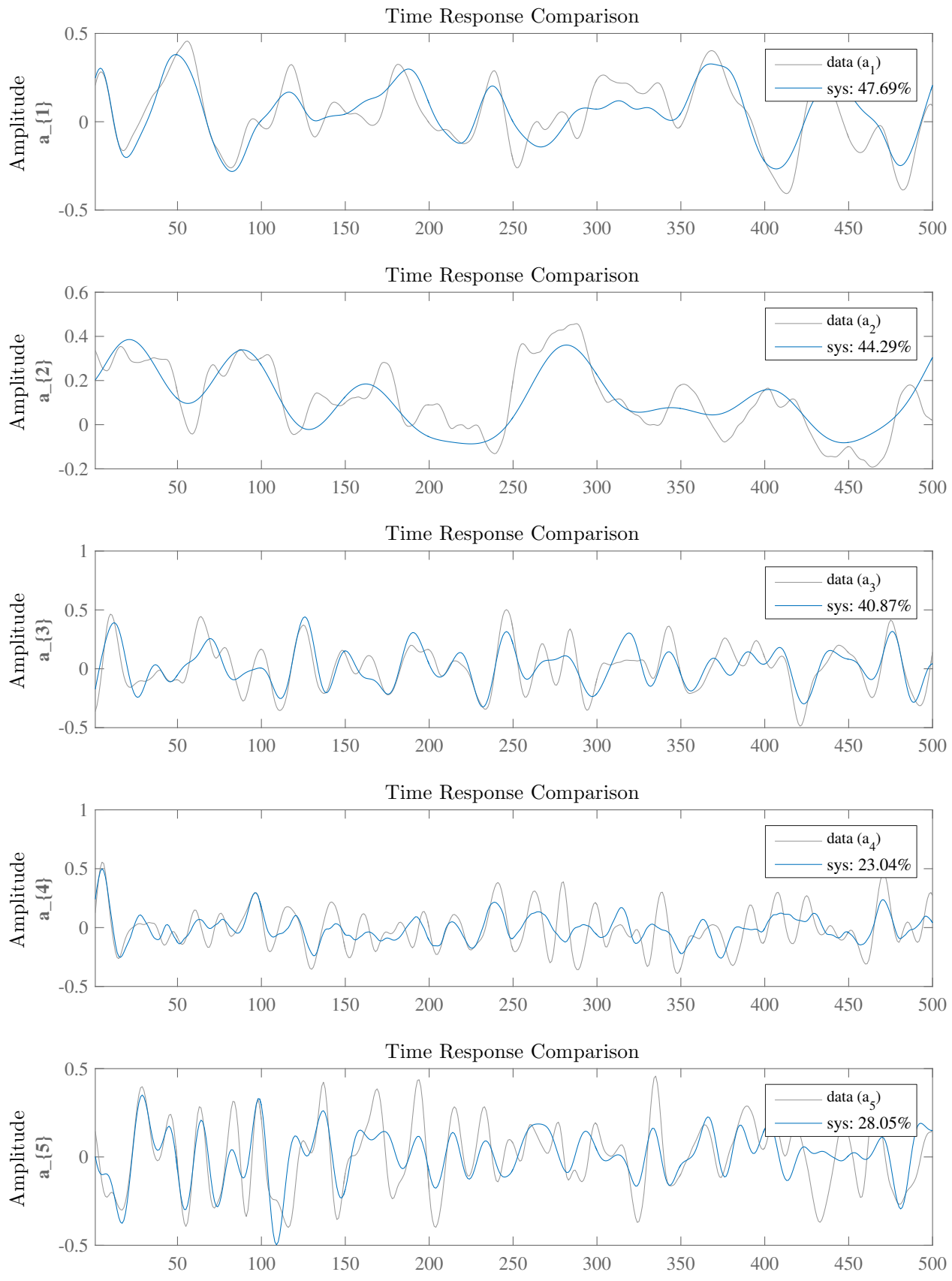

Figure 4.84: Comparison of mode coefficient from POD and that produced from the open-loop transfer functions. Only coefficients corresponding to modes 1-5 are shown. 
Table 4.6: Details for wake IV transfer functions including number of poles, zeros, and the goodness of fit relating $U_{\text {eff, } 4}$ to each mode coefficient.

\begin{tabular}{c|rrrrrr} 
Mode & 1 & 2 & 3 & 4 & 5 & 6 \\
Poles & 10 & 9 & 10 & 10 & 9 & 10 \\
Zeros & 7 & 5 & 7 & 9 & 8 & 8 \\
Fit (\%) & 47.69 & 44.29 & 40.87 & 23.04 & 28.05 & 24.26 \\
Mode & 7 & 8 & 9 & 10 & 11 & 12 \\
Poles & 10 & 10 & 10 & 8 & 10 & 9 \\
Zeros & 8 & 9 & 6 & 7 & 8 & 7 \\
Fit (\%) & 26.13 & 32.96 & 22.41 & 12.13 & 30.05 & 26.45
\end{tabular}

those related to the input velocity. Shown by the comparisons in Figure 4.84, the predictions via transfer functions do not match as well as for the isolated wind turbine. Decrease fit quality is presumed to arise from the nature of the effective inflow velocity. For a wind turbine in an array, the inflow is the far wake of a preceding device. While turbulence in the far wake is considered homogenous, at least compared to the near wake, it is still a significantly more complex flow than the unperturbed ABL. Details regarding wake IV transfer functions are described in Table 4.6

Transfer functions are used to generate initial conditions to recalibrate the LODS with a prediction horizon of 20 seconds. Corrected mode coefficients demonstrate considerably less deviation between the calculated and modeled mode coefficients as long as an input signal is available. Time-series of mode coefficients corrected with open-loop transfer functions are shown in Figure 4.85. The agreement seen between the calculated and modeled coefficients is encouraging and provides a path along which the next wake in the array may be modeled. A velocity signal is calculated combining the POD modes and the modeled coefficients according to Equation (2.19). The 

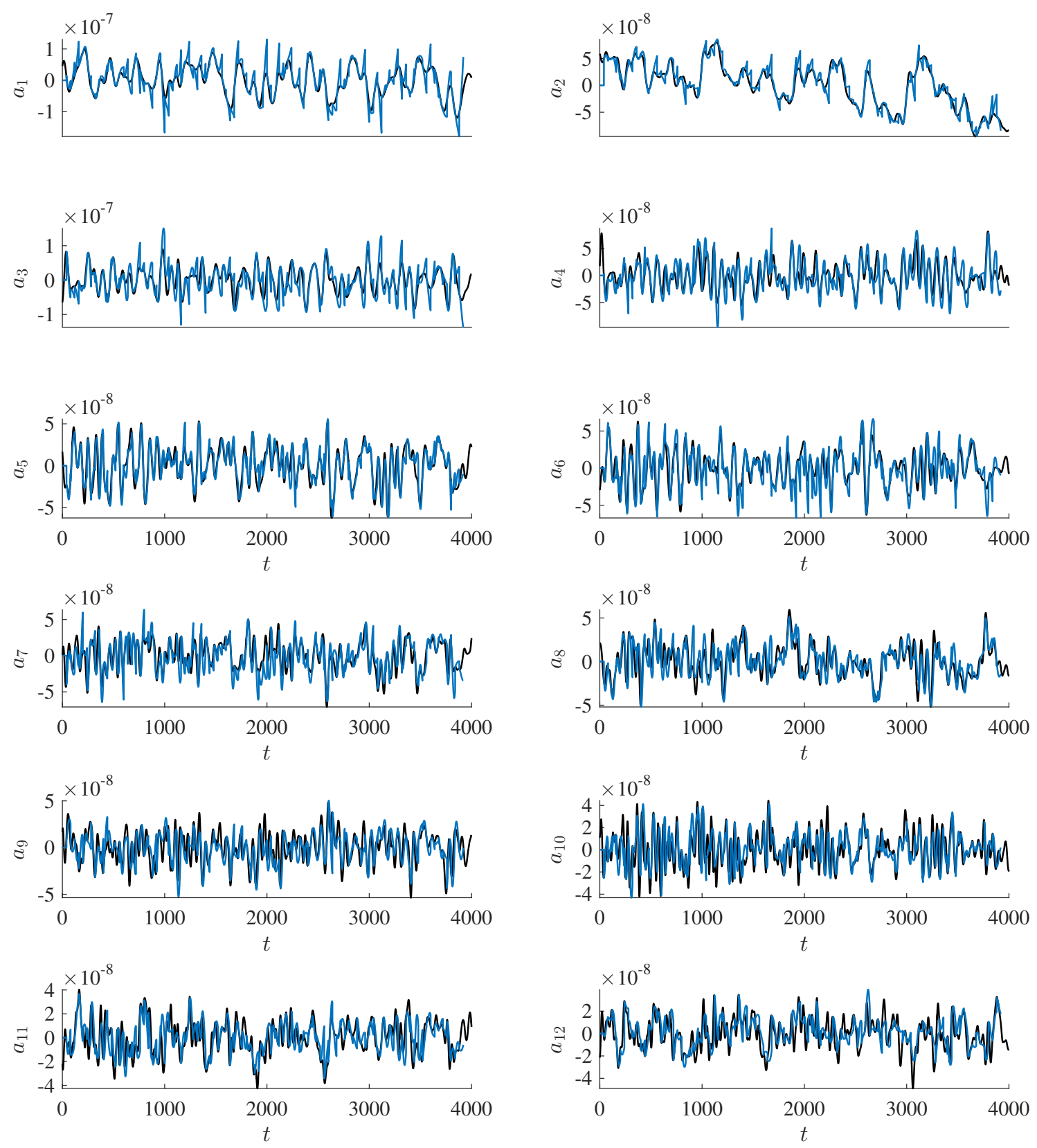

Figure 4.85: Time-series comparison of calculated (black) and modeled (blue) coefficients after transfer function recalibration.

resulting signal is then used as the input for wake V. Figure 4.86 compares the velocity signals used to calibrate wake V. In the figure, the black profile represents the inflow 
signal computed from the LES velocity fields directly. The blue line shows the result of the LODS model in wake IV. As seen for the wake of the isolated wind turbine, the model here shows the filtering effect from truncating the mode basis. Large features of the signal are captured by the model although there is a slight time delay in the predictions.

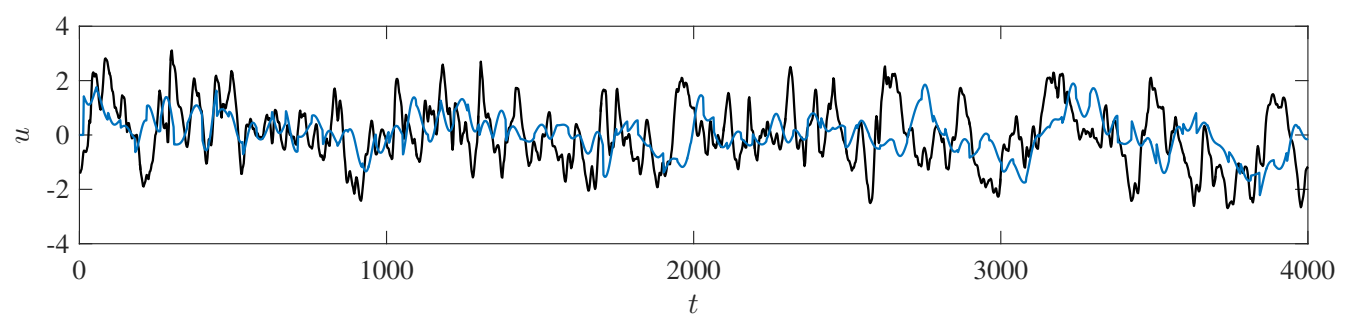

Figure 4.86: Effective inflow velocity signals provided to a wind turbine in the fifth row. The trend in black is calculated from the LES directly, the trend in blue results from the LODS model for wake IV.

Signals from Figure 4.86 are related to the mode coefficient time-series for wake $\mathrm{V}$ by a new series of open-loop transfer functions tuned in the same manner as before. Transfer functions and fit criteria are shown in Table 4.7. The specifications of each transfer function vary according to the input signal matched to the coefficients; for a given mode, the number of poles and zeros, and their respective values are distinct between the LES inflow signal and the model signal resulting from the wake IV LODS. The fit quality between the LES transfer functions varies between $17-62 \%$. For the transfer functions relating the wake IV LODS to the wake V POD coefficients, the range of the fit is approximately the same, although slight improvements are seen for particular modes. Increased values of the fit likely result from matching a filtered velocity signal to the coefficients.

Finally, predictions of wake V mode coefficients are shown in Figure 4.87. Predic- 
Table 4.7: Transfer function parameters for wake V. The first set of poles, zeros, and fit quality refer to the LES inflow signal. The second set refer to the LODS model from wake IV.

\begin{tabular}{c|rrr|rrr} 
Mode & poles & zeros & fit (\%) & poles & zeros & fit (\%) \\
\hline 1 & 9 & 8 & 47.31 & 10 & 3 & 47.23 \\
2 & 10 & 2 & 62.47 & 10 & 1 & 63.48 \\
3 & 9 & 8 & 30.13 & 10 & 7 & 26.13 \\
4 & 10 & 9 & 29.27 & 10 & 7 & 32.65 \\
5 & 10 & 9 & 35.21 & 10 & 9 & 26.02 \\
6 & 10 & 9 & 31.95 & 10 & 5 & 23.12 \\
7 & 10 & 7 & 29.55 & 10 & 3 & 18.26 \\
8 & 8 & 6 & 28.14 & 9 & 8 & 22.28 \\
9 & 10 & 9 & 25.97 & 10 & 7 & 25.69 \\
10 & 9 & 8 & 17.08 & 10 & 9 & 15.60 \\
11 & 10 & 8 & 41.70 & 10 & 9 & 42.44 \\
12 & 10 & 7 & 20.04 & 10 & 9 & 27.09
\end{tabular}

tions of the mode coefficients from the LODS deviate from their calculated values very quickly, suggesting that recycling information from one dynamical system to the next introduces instability. Sufficiently short prediction horizons, in this case 20 seconds, allow the transfer functions to return the modeled coefficients back to their validation trajectories but introduce noise. The figure shows that large features of $a_{1}$ and $a_{2}$ are reproduced well by the LODS. Higher order modes exhibit greater degrees of noise but remain centered on the calculated trends. Curiously, predictions of the highest included modes in the system $a_{11}$ and $a_{12}$ are on the same order of accuracy as the lowest ranking modes. 

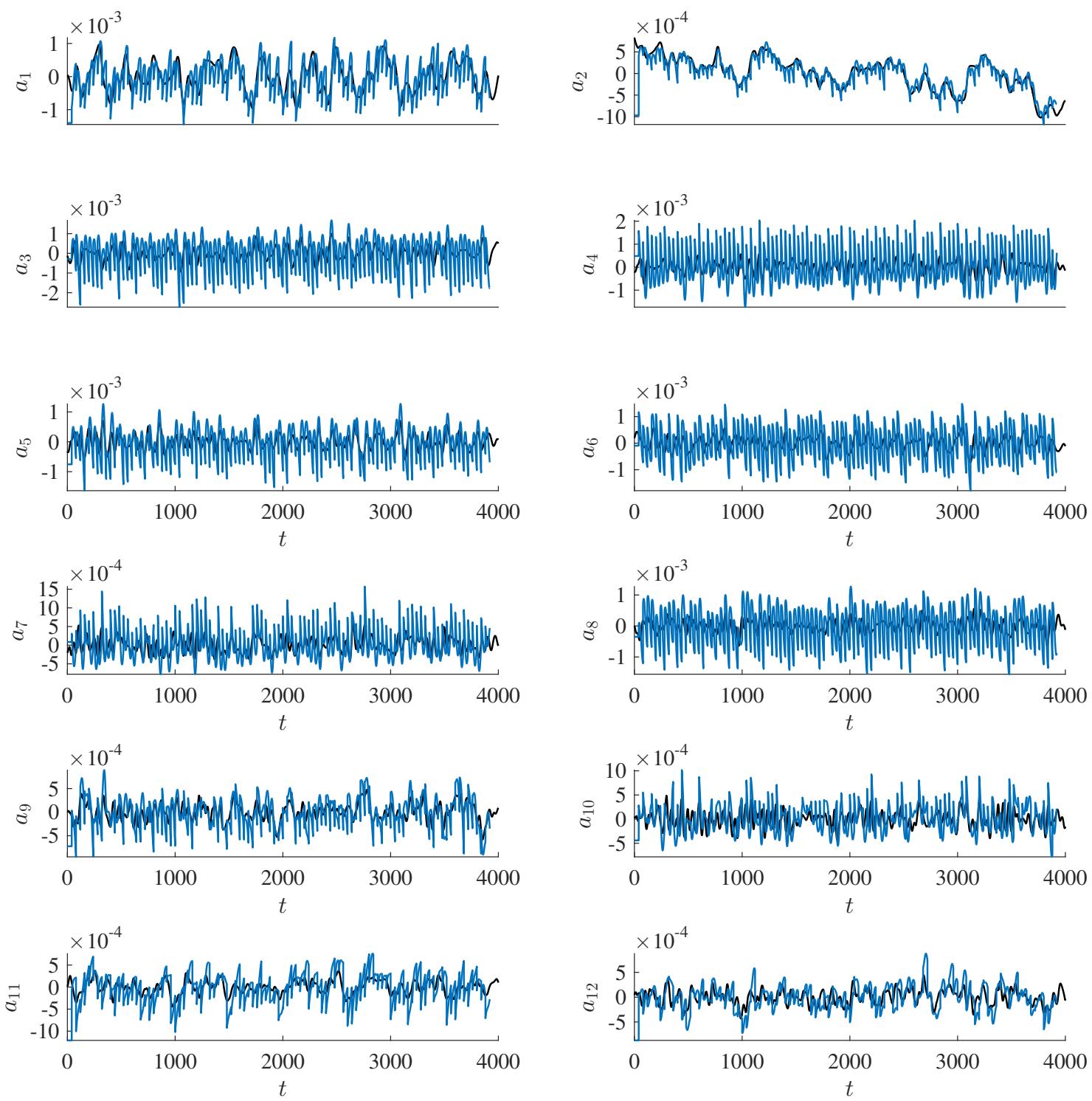

Figure 4.87: Mode coefficients for wake V. Series derived from the POD are shown in black, series from the wake V LODS are shown in blue.

\subsubsection{Conclusion}

Large eddy simulation of a wind turbine array is approached for analysis and the development of low-order dynamical systems with the intent to formulate a continuous time modeling and control scheme for wind turbines. Data for two wakes are extracted 
from the full simulation. The methodology developed in preceding sections is applied to the upstream wake, here following a turbine in the fourth row of the array. POD modes are used to define a LODS using the least-squares fit method.

Transfer functions relate the effective inflow velocity to the mode coefficients associated in the LODS. Each transfer function is defined to maximize the quality of fit between the modeled and calculated coefficients. The resulting dynamical system is composed of 12 modes and accounts for $46 \%$ of the turbulence kinetic energy in the LES of wake IV. Modes are combined with their respective coefficient predictions to reconstruct a velocity field in the wake. Dynamics predicted by the LODS are informed of the inflow field by resupplying the ODES with new initial conditions every 20 seconds.

Wake $\mathrm{V}$ is analyzed with an identical approach, with the exception that two input signals are tested; one calculated directly from the LES data and the other from the LODS prediction of wake IV. Transfer functions are calculated for each signal and exhibit differences in the number of poles and zeros of the system, as well as the fit between actual and predicted values. Recalibration with the transfer functions is undertaken at a prediction horizon of 20 seconds. This horizon is sufficient to return the predicted coefficient trajectory to their validation values calculated directly in the POD. Predictions beyond the input are not shown. Without a signal to offer the dynamical system, predicted modes are not expected to reflect incoming flow phenomena and rapidly become unstable.

The current modeling approach requires a statistical record for each wake. This requirement may be side-stepped for a fully-developed wind turbine array, where wake statistics are assumed to be periodic from row to row. When this is the case, POD modes may be recycled with each row and a significantly smaller statistical record is 
necessary. Additionally, adding modes to the statistical record that account for mismatch between the incoming flow and yaw orientation of the turbine would open a variable well suited for feedback control. Previous research (Chamorro et al., 2012) suggests that the fully developed region is reached beyond the fourth row of turbines in a large array, although validation of those results is not undertaken here, and POD modes are calculated for each wake individually.

Dynamical systems derived and tested above demonstrate that the current method is a viable path for continuous time control of a wind turbine array. The recalibration process requires little in the way of computational power and is able to provide renewed initial conditions to the system as fast as needed. Tuning and optimization of the transfer functions remains to be addressed. It is expected that optimization will lead to decreased instability in the coefficients and allow for extended prediction horizons. With a tuned dynamical system, the above method demonstrates the capability to daisy-chain wakes together and provides a means of predicting wake flows in a very large array with few monitoring signals. 


\section{Chapter 5}

\section{Conclusions}

\subsection{Review of findings}

Turbulent fluid dynamics in the wake of a wind turbine are analyzed with a range of techniques revealing an array of compelling physical insights and options for detailed development of accessible dynamical models. Structures evolving very near to the wind turbine rotor have been reputed to exhibit dependence of the phase orientation of the rotor blades, although these effects are greatly obscured when the flow incoming to a given wind turbine deviate from ideal conditions. Formulation of higher order statistics, termed tertiary stresses, exhibit structures relating to the passage of the rotor blades. The tensor of tertiary stresses represents the root-mean-square deviation of phase averaged turbulence statistics from their average-passage counterparts. Diagonal components of the tensor are interpreted as the deterministic analog to turbulence intensity.

Interestingly, the deterministic stresses themselves are not significant in the wind turbine wake, as they are in other forms of turbomachinery. In the case of gas turbines, where deterministic stresses are of the same order of magnitude as the turbulent con-

tributions, the flow field is excited by a fixed geometry and set of operating conditions 
that constantly add energy to a particular distribution of scales. In contrast, wind turbine wakes represent a sink of energy in the governing equations, and the resulting turbulence seeks to redistribute energy into momentum deficit regions of the flow. This fundamental difference in behavior is the hypothesized cause for the dissimilarity in significance of turbulent, deterministic, and, presumably, tertiary contributions to the energy balance.

Snapshot proper orthogonal decomposition is coupled with anisotropy tensor invariant analysis illustrating the ability of reduced-order descriptions to reproduce complexity in turbulent flows. An extensive error analysis was conducted regarding the anisotropy of POD-filtered turbulence for a wind turbine wake and a fully-developed channel flow. Lumley triangles mapping the anisotropy tensor invariant space composed with the low-rank POD modes exhibit exaggerated values of $\eta$ and $\xi$. Contrarily, the intermediate- and high-rank POD modes contribute much more to small-scale and homogeneous turbulence, and result in smaller magnitudes of $\eta$ and $\xi$, confirming that they express mainly isotropic turbulence structures. The propagation of root-meansquare error between reduced-order descriptions and the full statistics demonstrates that a turbulence field represented by highly anisotropic turbulence is accurately represented with very few modes.

Such descriptions exclude homogeneously distributed, isotropic turbulence that is easily accounted for with a correction constant. Residual error between the corrected POD approximations and the original turbulence statistics is between $25 \%$ and $40 \%$ lower than the uncorrected low-order description. Perhaps more interesting theoretically, correction of the reduced-order description effectively rebalances the magnitudes of the Reynolds stresses and demonstrates anisotropy tensor invariants much 
closer to those derived from the full statistics than uncorrected flow descriptions. In the cases of severe basis truncation, the POD descriptions are able to account for only one- or two-dimensional turbulence. Correction with constant coefficients enables the representation of three-dimensional turbulence even for severe basis truncations.

The snapshot POD is a well-tested analysis tool that is regularly employed to assess the spatial distribution of turbulence structures important to a flow field. A new modification of the method is developed here that allows spatial evolution of POD modes to be characterized for further model reduction and flow description. The nested application of the POD undertaken in the analysis of a wind turbine wake reconciles POD bases composed of snapshot sets at successive downstream locations in the wake. The proposed formulation relies on the assumption that for POD bases of the same size and issuing from the same flow, modes at identical rank must evolve spatially to accommodate evolution of the turbulence field they represent. The relationship of the POD modes is difficult to assess with traditional statistical means as the modes are agnostic to algebraic sign, relying on the associated mode coefficients for sign information. However, the secondary eigenvalue decomposition of the double POD seeks common projections of the proper orthogonal modes and ignores their algebraic sign altogether. The sub-modal bases in the wind turbine wake demonstrate the anticipated organization and provide a means of filtering the POD modes to a base structure and successively more nuanced corrections whose importance in the wake is communicated through a spatially explicit series of coefficients.

Beyond acting as a filter flow turbulent flow description, the POD represents and attractive pathway to modal forms of low-order modeling. The favored approach historically has been through the Galerkin projection wherein the governing behavior law 
is projected onto a truncated set of basis modes. The optimality of the POD in describing the energy of the flow means that POD modes are well-suited to forming dynamical models. However, Galerkin projection tends toward model instability for systems with high variability, as is the case in the turbulent flows tested here. In an effort to simultaneously add stability and complexity to the dynamical model posed for the fully-developed channel flow by the Galerkin projection, the particular basis selected for projection was varied to include intermediate and high rank POD modes. Resulting dynamical systems show a variety of different behaviors, ranging from increased complexity in some cases to behavior similar to critical damping of dynamical components in others.

Alternate formulations of low-order dynamical systems have been proposed that are able to handle systems with high turbulence intensity such as the channel flow and wind turbine wakes. A least-squares polynomial fit method is distinguished from the Galerkin Projection in that it derives the dynamics of the system through the POD coefficients rather than the modes. The method was extensively explored in the current work as it applies to a fully-developed channel flow, testing its ability to make accurate representations of the flow physics at arbitrarily long time intervals. In defining polynomial coefficients that couple the dynamic evolution of POD mode coefficients, the method is able to resolve times series that closely match the coefficients time derivatives. The resulting system of ordinary differential equations are solved numerically, eventually deviate from the trajectories outlined by the POD, and ultimately grow unstable. Deviation from the original trajectories is inevitable but may be mitigated by increasing the size of the input mode basis or by tightening numerical tolerances, at the cost of computational efficiency. 
Through a recalibration process that matches mode coefficients after specified time intervals, the system is able to make predictions at times far beyond the original input. Randomization of the mode coefficient matched in the recalibration process prevents the system from falling into a closed orbit, where the predicted coefficients eventually repeat, and limit the system to a subspace of the full dynamics. The modeled mode coefficients are distinct from, but exhibit the dynamics within the bounds of their respective POD equivalents. A result of the abrupt recalibration process, discontinuities are introduced to the modeled velocity time series, that eventually appear in energy spectra as high frequency noise. The long time records produced in the loworder dynamical system provide a larger set of samples over which statistics may be calculated. Spectra and spatially-averaged profiles resulting from the dynamical system with recalibration more closely match the full channel flow DNS than the reducedorder description from the POD.

Low-order modeling is particularly attractive for wind turbine wakes, which relate directly to the power production of the turbines and exhibit a large range of scales. The complexity of wind turbine wakes has made them notoriously difficult to fully characterize with computationally efficient Reynolds-averaged Navier-Stokes models. Instead, more complex simulation schemes such as large eddy simulations are favored for exploration of wake physics. While able to better represent the dynamics of wind turbine wakes, large eddy simulations are too expensive and take too long to be used in wind farm design or control. The least-squares method employed for the channel flow is extended to LES data for a wind turbine wake in isolation, demonstrating that the complex physics of the wake are within reach of such low-dimensional schemes.

The low-order dynamical system for the wind turbine wake offers an efficient 
means of modeling that includes the large-scale turbulence attributed to rotor blade fatigue and fluctuations in power production. A different approach to recalibration of the dynamical system was taken for the wake than for the channel flow, with the goal of tuning the wake model to reflect incoming flow events. In order to provide the wake model with information about upstream activity, a virtual sensor was employed, wherein the effective inflow velocity to the rotor was calculated as a time series that extended beyond the data used to define the constituent modes. The input signal was correlated to the mode coefficients of the LODS using a series of open-loop transfer functions. Transfer functions were trained for one quarter of the total simulation time available, reserving the remainder to be employed as validation data. The low-order system was supplied with refreshed initial conditions, and is able to match the validation data with nearly zero error, provided that the period between recalibration is sufficiently small.

\subsection{Outlook}

Given the rate at which wind energy is being adopted domestically and internationally, the average underperformance of wind farms poses a significant problem. Maximizing the output of every installed wind turbine will be necessary to meet the ambitious energy goals in a meaningful way. Beyond the obvious benefit of increasing the portion of our energy budget gained from low-carbon and low-water methods, improving the monitoring and control methods used to operate wind farms will lead to decreased wear on mechanical systems, extending the life cycles of turbines and making wind energy more economical. The scale at which wind energy is being adopted means that single digit improvements of the capacity factor of wind energy, equate to generous 
fiscal margins that will not be long overlooked. Dynamical systems modeling of wind turbine wakes undertaken above represent one possible path toward closing the gap between installed and actual wind energy production.

The above development aims the analysis of wind turbine wakes toward the frequently cited goals of optimal design and control of large arrays. Efficient lowdimensional models are among the tools that will be required to meet current and future energy goals while meeting environmental and economic constraints. Tertiary stresses defined above offer a novel view of the variability of the turbulent wakes that compliment other conditional statistics such as quadrant or octant analysis. Energy transport mechanisms in the near wake differ from the wake as a whole and are expected to show more influence of structures including tip vortices that might be characterized with the tertiary stress tensor. Kinetic energy flux via tertiary stresses may also be related to vibrational modes and flexure of rotor blades, attributed to fatigue and failure of wind turbines in large arrays.

Low-dimensional models like those proposed herein represent a possible path for continuous monitoring of wind turbines and wakes. The ability to tune the model to an incoming velocity signal means that the model could be iteratively linked in a wind turbine array setting, shown in Figure 5.1. In the modified flowchart, the complete modeling procedure is contained in the blue box, excluding only the input velocity signal and the output wake prediction. When the wake flow output by the reducedorder model is used to make predictions of following wind turbines, power predictions for a full array become available. Additionally, feedback may then be added to the system, such as the yaw angle of the wind turbine $(\theta)$ and the blade pitch $(\beta)$ in order to maintain optimal operating conditions for all turbines in the array. 


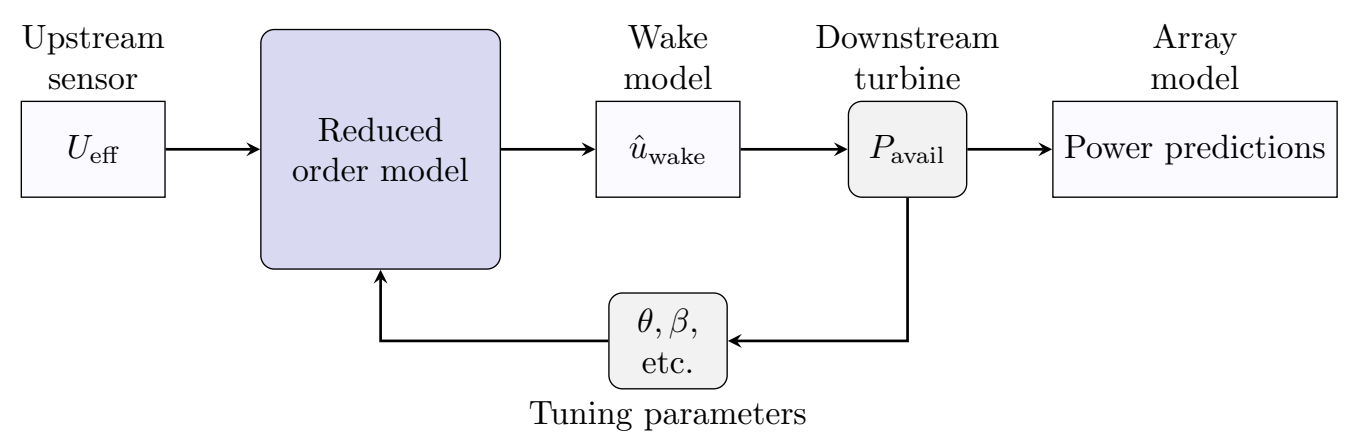

Figure 5.1: Example control scheme for a wind turbine in a large array. This scheme could be nested and account for many rows of wind turbines to provide global array control with very few inputs.

Modeling and control using low-order dynamical systems as proposed here will require further development of the transfer functions used to supply refreshed initial conditions to the reduced-order model. The system would greatly benefit from testing a range of possible inputs that a turbine may experience in real operating conditions, including strong gusts or high inflow turbulence intensity. The control parameters will also need to be redefined for wind turbine wakes in large arrays, where wake dynamics, and thus the evolution of modes and coefficients will differ from those presented above. Expanding the POD to include a range of operating conditions including yaw misalignment or effects of blade pitch on the wake would further benefit a control scenario like the one presented in the figure.

Beyond detailing wind turbine wakes, the POD has received a fair amount of development in the current work. The relationship between reduced-order descriptions from truncated mode bases and the anisotropy tensor invariants will be important in theoretical considerations moving forward. Many forms of model correction could be derived through the anisotropy tensor invariants and POD. The non-linear relationship between the Reynolds stress tensor and the anisotropy tensor invariants mean there is 
no intuitive combination of invariants that recovers the original data. However, there may be a path to describing the turbulence field through inverse eigenvalue decomposition methods, from which there are many to choose, each with particular benefits and drawbacks in terms of ease of calculation and accuracy of results.

Either by way of inverse decomposition or other means, a more general approach to defining correction terms would be of great benefit to the POD. Considering the gains detailed above, use of simple correction factors to rebalance the Reynolds stress tensor or to account for energy excluded in the truncation process is of obvious benefit. In cases of severe model truncation, the correction goes so far as to reintroduce three-dimensionality to the modeled turbulence field. With insight as to the expected balance of energy in the Reynolds stress tensor, it may be possible to find a universal approach to defining corrections in an a priori sense rather than an ad hoc one.

The double POD combines spatial and temporal eigenvalue decompositions, and in the process is able to offer a full order of magnitude further model reduction over the snapshot POD. That it can reconcile modes from distinct locations in space provides a means of defining models dedicated to mode evolution, that could effectively operate within the scope of dynamical systems. A cursory attempt to apply the least-squares polynomial fit methods used above show that the DPOD is indeed a possible path forward in spatial modeling, beyond simply filtering noise out of the POD modes. The bridge from spatial modeling analogous to the LODS above to simultaneous dynamical evolution requires further development before the DPOD reaches its full potential.

Analysis methods developed in the present work are applicable to an enormous body of turbulent flow scenarios. Tertiary stresses will be of benefit to any flow exhibiting periodicity; they are clearly important in turbomachinery and turbulent en- 
ergy fluxes. Tertiary stress analysis may provide a new means of characterizing vortex shedding or flow instability as well. The DPOD is well-suited to application of any flow expected to evolve spatially, such as jets, shearing flows, or developing boundary layers. Nested decomposition may lead to representations of spatially evolving flows analogous to similarity solutions and is expected to reveal intrinsic physical mechanisms that are otherwise difficult to identify. Low-order models derived from the DPOD will open new channels to turbulence modeling. Simultaneous spatial and dynamical modeling with DPOD modes will provide data to desired resolution with an extremely small modal basis. 


\section{Bibliography}

J. J. Adamczyk. Aerodynamic analysis of multistage turbomachinery flows in support of aerodynamic design. Journal of Turbomachinery, 122(2):189-217, 2000.

J. F. Ainslie. Calculating the flowfield in the wake of wind turbines. Journal of Wind Engineering and Industrial Aerodynamics, 27:213-224, 1988.

J. D. Albertson and M. B. Parlange. Natural integration of scalar fluxes from complex terrain. Advances in Water Resources, 23(3):239-252, 1999.

N. Aubry, P. Holmes, J. L. Lumley, and E. Stone. The dynamics of coherent structures in the wall region of a turbulent boundary layer. Journal of Fluid Mechanics, 192: 115-173, 1988.

N. Aubry, R. Guyonnet, and R. Lima. Spatiotemporal analysis of complex signals: theory and applications. Journal of Statistical Physics, 64(3-4):683-739, 1991.

R. Barthelmie, O. Rathmann, S. Frandsen, K. Hansen, E. Politis, J. Prospathopoulos, K. Rados, D. Cabezon, W. Schlez, J. Phillips, A. Neubert, J. G. Schepers, and S. P. van der Pijl. Modelling and measurements of wakes in large wind farms. Journal of Physics: Conference series, 75, 2007.

R. J. Barthelmie, K. Hansen, S. T. Frandsen, O. Rathmann, J. Schepers, W. Schlez, J. Phillips, K. Rados, A. Zervos, E. Politis, et al. Modelling and measuring flow and wind turbine wakes in large wind farms offshore. Wind Energy, 12(5):431-444, 2009.

D. Bastine, B. Witha, M. Wächter, and J. Peinke. Towards a simplified dynamicwake model using pod analysis. Energies, 8(2):895-920, 2015.

R. A. Białecki, A. J. Kassab, and A. Fic. Proper orthogonal decomposition and modal analysis for acceleration of transient fem thermal analysis. International journal for numerical methods in engineering, 62(6):774-797, 2005.

L. Biferale and I. Procaccia. Anisotropy in turbulent flows and in turbulent transport. Physics reports, 414(2):43-164, 2005. 
E. Bou-Zeid, C. Meneveau, and M. Parlange. A scale-dependent lagrangian dynamic model for large eddy simulation of complex turbulent flows. Physics of Fluids (1994present), 17(2):025105, 2005.

C. Braud, D. Heitz, G. Arroyo, L. Perret, J. Delville, and J.-P. Bonnet. Low-dimensional analysis, using pod, for two mixing layer-wake interactions. International journal of heat and fluid flow, 25(3):351-363, 2004.

S. L. Brunton and B. R. Noack. Closed-loop turbulence control: Progress and challenges. Applied Mechanics Reviews, 67(5):050801, 2015.

R. B. Cal, J. Lebrón, L. Castillo, H. S. Kang, and C. Meneveau. Experimental study of the horizontally averaged flow structure in a model wind-turbine array boundary layer. Journal of Renewable and Sustainable Energy, 2:013106, 2010.

M. Calaf, C. Meneveau, and J. Meyers. Large eddy simulation study of fully developed wind-turbine array boundary layers. Physics of Fluids, 22:015110, 2010.

L. P. Chamorro and F. Porté-Agel. A wind-tunnel investigation of wind-turbine wakes: Boundary-layer turbulence effects. Boundary Layer Metrology, 132:129-149, 2009.

L. P. Chamorro and F. Porté-Agel. Turbulent flow inside and above a wind farm: A windtunnel study. Energies, 4(11):1916-1936, 2011 a.

L. P. Chamorro and F. Porté-Agel. Turbulent flow inside and above a wind farm: A wind-tunnel study. Energies, 4(11):1916-1936, 2011b. ISSN 1996-1073. doi: 10.3390/ en4111916. URL http: //www.mdpi . com/1996-1073/4/11/1916.

L. P. Chamorro, R. Arndt, and F. Sotiropoulos. Turbulent flow properties around a staggered wind farm. Boundary-Layer Meteorology, 141(3):349-367, 2011. ISSN 0006-8314. doi: 10.1007/s10546-011-9649-6. URL http://dx.doi.org/10.1007/ s10546-011-9649-6.

L. P. Chamorro, R. Arndt, and F. Sotiropoulos. Reynolds number dependence of turbulence statistics in the wake of wind turbines. Wind Energy, 15(5):733-742, 2012. ISSN 1099-1824. doi: 10.1002/we.501. URL http://dx . doi .org/10.1002/we. 501.

K.-S. Choi and J. L. Lumley. The return to isotropy of homogeneous turbulence. Journal of Fluid Mechanics, 436(1):59-84, 2001.

J. H. Citriniti and W. K. George. Reconstruction of the global velocity field in the axisymmetric mixing layer utilizing the proper orthogonal decomposition. Journal of Fluid Mechanics, 418:137-166, 2000. 
A. Crespo, J. Hernández, and S. Frandsen. Survey of modelling methods for wind turbine wakes and wind farms. Wind Energy, 2:1-24, 1999.

J. D’adamo, N. Papadakis, E. Memin, and G. Artana. Variational assimilation of pod low-order dynamical systems. Journal of Turbulence, (8):N9, 2007.

S. Emeis and S. Frandsen. Reduction of horizontal wind speed in a boundary layer with obstacles. Boundary-Layer Meteorology, 64(3):297-305, 1993.

J. Favier, L. Cordier, and A. Kourta. Accurate pod reduced-order models of separated flows. Under consideration for publication in Physics of Fluids, 2008.

J. A. Fike. Construction of reduced order models for the non-linear navier-stokes equations using the proper orthogonal decomposition (pod)/galerkin method. Sandia National Laboratories Report, SAND, (2013-7051), 2013.

S. Frandsen, R. Barthelmie, S. Pryor, O. Rathmann, S. Larsen, J. Højstrup, and M. Thøgersen. Analytical modelling of wind speed deficit in large offshore wind farms. Wind energy, 9(1-2):39-53, 2006.

L. Fried, S. Sawyer, S. Shukla, and L. Qiao. Global wind report annual market update 2013'. gwec, 2015.

W. K. George. Lectures in turbulence for the 21st century. Chalmers University of Technology, 2009.

Global Wind Energy Council. Global wind report-annual market update 2014, 2014.

R. Gómez-Elvira, A. Crespo, E. Migoya, F. Manuel, and J. Hernández. Anisotropy of turbulence in wind turbine wakes. Journal of wind engineering and industrial aerodynamics, 93(10):797-814, 2005.

J. Graham, M. Lee, N. Malaya, R. Moser, G. Eyink, C. Meneveau, K. K. R. Burns, and A. Szalay. Turbulent channel flow data set. Johns Hopkins University, 2013.

W. Graham, J. Peraire, and K. Tang. Optimal control of vortex shedding using low-order models. part i-open-loop model development. International Journal for Numerical Methods in Engineering, 44(7):945-972, 1999.

N. Hamilton and R. B. Cal. Anisotropy of the reynolds stress tensor in the wakes of wind turbine arrays in cartesian arrangements with counter-rotating rotors. Physics of Fluids (1994-present), 27(1):015102, 2015. 
N. Hamilton, M. Tutkun, and R. B. Cal. Anisotropy invariant analysis and the proper orthogonal decomposition of turbulent flows. Physical Review: Fluids, (submitted for publication March, 2016).

N. Hamilton, H.-S. Kang, C. Meneveau, and R. B. Cal. Statistical analysis of kinetic energy entrainment in a model wind turbine array boundary layer. Journal of Renewable and Sustainable Energy, 4(6):063105-063105, 2012. doi: http://dx.doi.org/ $10.1063 / 1.4761921$.

N. Hamilton, M. Melius, and R. B. Cal. Wind turbine boundary layer arrays for cartesian and staggered configurations-part i, flow field and power measurements. Wind Energy, 18(2):277-295, 2015a.

N. Hamilton, M. Tutkun, and R. B. Cal. Wind turbine boundary layer arrays for cartesian and staggered configurations-part II, low-dimensional representations via the proper orthogonal decomposition. Wind Energy, 18(2):297-315, 2015b.

N. Hamilton, M. Tutkun, and R. B. Cal. Low-order representations of the canonical wind turbine array boundary layer via double proper orthogonal decomposition. Physics of Fluids (1994-present), 28(2):025103, 2016.

N. Hamilton, M. Tutkun, and R. B. Cal. Low-order dynamical systems modeling of fullydeveloped turbulent channel flow. Physica D: Nonlinear Phenomena, submitted for publication May, 2016.

K. Hasselmann. Pips and pops: The reduction of complex dynamical systems using principal interaction and oscillation patterns. Journal of Geophysical Research: Atmospheres (1984-2012), 93(D9):11015-11021, 1988.

H. Hu, Z. Yang, and P. Sarkar. Dynamic wind loads and wake characteristics of a wind turbine model in an atmospheric boundary layer wind. Experiments in fluids, 52(5): 1277-1294, 2012.

Wake stabilization using POD Galerkin models with interpolated modes, 2005. IEEE.

M. Ilak and C. W. Rowley. Modeling of transitional channel flow using balanced proper orthogonal decomposition. Physics of Fluids (1994-present), 20(3):034103, 2008.

A. Jimenez, A. Crespo, E. Migoya, and J. Garcia. Advances in large-eddy simulation of a wind turbine wake. In Journal of Physics: Conference Series, volume 75, page 012041. IOP Publishing, 2007.

J. Kim, P. Moin, and R. Moser. Turbulence statistics in fully developed channel flow at low reynolds number. Journal of fluid mechanics, 177:133-166, 1987. 
J. Kostas, J. Soria, and M. Chong. A comparison between snapshot pod analysis of piv velocity and vorticity data. Experiments in Fluids, 38(2):146-160, 2005.

K. Kunisch and S. Volkwein. Galerkin proper orthogonal decomposition methods for a general equation in fluid dynamics. SIAM Journal on Numerical analysis, 40(2): 492-515, 2002.

M. P. Laan, N. N. Sørensen, P.-E. Réthoré, J. Mann, M. C. Kelly, N. Troldborg, J. G. Schepers, and E. Machefaux. An improved $k \epsilon$ model applied to a wind turbine wake in atmospheric turbulence. Wind Energy, 18(5):889-907, 2015.

M. Lee, N. Malaya, and R. D. Moser. Petascale direct numerical simulation of turbulent channel flow on up to 786k cores. In Proceedings of the International Conference on High Performance Computing, Networking, Storage and Analysis, page 61. ACM, 2013.

S. Leonardi, P. Orlandi, L. Djenidi, and R. A. Antonia. Structure of turbulent channel flow with square bars on one wall. International journal of heat and fluid flow, 25 (3):384-392, 2004.

Y. Li, E. Perlman, M. Wan, Y. Yang, C. Meneveau, R. Burns, S. Chen, A. Szalay, and G. Eyink. A public turbulence database cluster and applications to study lagrangian evolution of velocity increments in turbulence. Journal of Turbulence, (9):N31, 2008.

L. Lignarolo, D. Ragni, C. Krishnaswami, Q. Chen, C. Simão Ferreira, and G. Van Bussel. Experimental analysis of the wake of a horizontal-axis wind-turbine model. Renewable Energy, 2014.

L. Lignarolo, D. Ragni, C. S. Ferreira, and G. van Bussel. Turbulent mixing in wind turbine and actuator disc wakes: experiments and POD analysis. American Institute of Aeronautics and Astronautics, 2015/05/21 2015. doi: doi:10.2514/6.2015-0223. URL http: //dx.doi .org/10.2514/6.2015-0223.

S. Lindenberg, B. Smith, K. O’Dell, et al. 20\% wind energy by 2030. National Renewable Energy Laboratory (NREL), US Department of Energy, Renewable Energy consulting Services, Energetics Incorporated, 2008.

J. L. Lumley. The structure of inhomogeneous turbulent flows. Atmospheric turbulence and radio wave propagation, pages 166-178, 1967.

F. R. Menter, A. V. Garbaruk, and Y. Egorov. Explicit algebraic reynolds stress models for anisotropic wall-bounded flows. In Progress in Flight Physics, volume 3, pages 89-104. EDP Sciences, 2012. 
P. Mestayer. Local isotropy and anisotropy in a high-reynolds-number turbulent boundary layer. Journal of Fluid Mechanics, 125:475-503, 1982.

J. Meyers and C. Meneveau. Optimal turbine spacing in fully developed wind-farm boundary layers. Wind Energy, 15:305-317, 2012.

J. Meyers and C. Meneveau. Flow visualization using momentum and energy transport tubes and applications to turbulent flow in wind farms. Journal of Fluid Mechanics, 715:335-358, 2013.

D. Milborrow and J. Ainslie. Intensified study of wake effects behind single turbines and in wind turbine wakes. Final Report of IEA, Annex IX, 1992.

C.-H. Moeng. A large-eddy-simulation model for the study of planetary boundary-layer turbulence. Journal of the Atmospheric Sciences, 41(13):2052-2062, 1984.

N. G. Mortensen and E. L. Petersen. Influence of topographical input data on the accuracy of wind flow modelling in complex terrain. In EWEC-CONFERENCE-, pages 317-320. BOOKSHOP FOR SCIENTIFIC PUBLICATIONS, 1997.

B. R. Noack, K. Afanasiev, M. Morzynski, G. Tadmor, and F. Thiele. A hierarchy of lowdimensional models for the transient and post-transient cylinder wake. Journal of Fluid Mechanics, 497:335-363, 2003.

B. R. Noack, P. Papas, and P. A. Monkewitz. The need for a pressure-term representation in empirical galerkin models of incompressible shear flows. Journal of Fluid Mechanics, 523:339-365, 2005.

S. A. Orszag and Y.-H. Pao. Numerical computation of turbulent shear flows. In Turbulent Diffusion in Environmental Pollution, volume 1, pages 225-236, 1974.

E. Perlman, R. Burns, Y. Li, and C. Meneveau. Data exploration of turbulence simulations using a database cluster. In Proceedings of the 2007 ACM/IEEE conference on Supercomputing, page 23. ACM, 2007.

L. Perret, P. Braud, C. Fourment, L. David, and J. Delville. 3-component acceleration field measurement by dual-time stereoscopic particle image velocimetry. Experiments in Fluids, 40(5):813-824, $2006 \mathrm{a}$.

L. Perret, E. Collin, and J. Delville. Polynomial identification of pod based low-order dynamical system. Journal of Turbulence, (7):N17, $2006 \mathrm{~b}$.

S. Pope. Turbulent flows. Cambridge university press, 2000. 
M.-L. Rapún and J. M. Vega. Reduced order models based on local pod plus galerkin projection. Journal of Computational Physics, 229(8):3046-3063, 2010.

O. Rathmann, S. Frandsen, and R. Barthelmie. Wake modeling for intermediate and large wind farms. 2007.

D. Rempfer. On low-dimensional galerkin models for fluid flow. Theoretical and Computational Fluid Dynamics, 14(2):75-88, 2000.

W. Reynolds and A. Hussain. The mechanics of an organized wave in turbulent shear flow. part 3. theoretical models and comparisons with experiments. Journal of Fluid Mechanics, 54(02):263-288, 1972.

W. Rodi and G. Bergeles. Improved algebraic reynolds stress model for engineering. Engineering Turbulence Modelling and Experiments-3, page 121, 2012.

R. S. Rogallo and P. Moin. Numerical simulation of turbulent flows. Annual Review of Fluid Mechanics, 16(1):99-137, 1984.

J. C. Rotta. Statistische theorie nichthomogener turbulenz. Zeitschrift für Physik, 129 (6):547-572, 1951.

S. G. Saddoughi and S. V. Veeravalli. Local isotropy in turbulent boundary layers at high reynolds number. Journal of Fluid Mechanics, 268:333-372, 1994.

V. Sharma, M. Calaf, M. Lehning, and M. Parlange. Time-adaptive wind turbine model for an les framework. Wind Energy, 2015.

S. Siegel, K. Cohen, J. Seidel, and T. McLaughlin. Short time proper orthogonal decomposition for state estimation of transient flow fields. AIAA paper, 296:2005, 2005.

S. Siegel, K. Cohen, J. Seidel, and T. McLaughlin. State estimation of transient flow fields using double proper orthogonal decomposition (dpod). In Active Flow Control, pages 105-118. Springer, 2007.

S. G. Siegel, J. Seidel, C. Fagley, D. Luchtenburg, K. Cohen, and T. McLaughlin. Lowdimensional modelling of a transient cylinder wake using double proper orthogonal decomposition. Journal of Fluid Mechanics, 610:1-42, 2008.

L. Sirovich. Turbulence and the dynamics of coherent structures. Quarterly of applied mathematics, 45:561-571, 1987.

R. Smalley, S. Leonardi, R. Antonia, L. Djenidi, and P. Orlandi. Reynolds stress anisotropy of turbulent rough wall layers. Experiments in fluids, 33(1):31-37, 2002. 
A. Smith, T. Stehly, and W. Musial. 2014-2015 offshore wind technologies market report. Technical report, NREL (National Renewable Energy Laboratory (NREL), 2015.

W. D. Smyth and J. N. Moum. Anisotropy of turbulence in stably stratified mixing layers. Physics of Fluids, 12:1343, 2000.

H. Snel. Review of aerodynamics for wind turbines. Wind Energy, 6(3):203-211, 2003. ISSN 1099-1824. doi: 10.1002/we.97. URL http://dx.doi .org/10.1002/we.97.

J. Sørensen, W. Shen, and X. Munduate. Analysis of wake states by a full-field actuator disc model. Wind Energy, 1(2):73-88, 1998.

J. N. Sørensen, R. Mikkelsen, D. S. Henningson, S. Ivanell, S. Sarmast, and S. J. Andersen. Simulation of wind turbine wakes using the actuator line technique. Philosophical Transactions of the Royal Society of London A: Mathematical, Physical and Engineering Sciences, 373(2035):20140071, 2015.

G. Tadmor, O. Lehmann, B. R. Noack, L. Cordier, J. Delville, J.-P. Bonnet, and M. Morzyński. Reduced-order models for closed-loop wake control. Philosophical Transactions of the Royal Society of London A: Mathematical, Physical and Engineering Sciences, 369(1940):1513-1524, 2011.

A. A. Townnsend. Local isotropy in the turbulent wake of a cylinder. Australian Journal of Chemistry, 1(2):161-174, 1948.

F. Tröltzsch and S. Volkwein. Pod a-posteriori error estimates for linear-quadratic optimal control problems. Computational Optimization and Applications, 44(1):83-115, 2009.

M. Tutkun, P. B. V. Johansson, and W. K. George. Three-component vectorial proper orthogonal decomposition of axisymmetric wake behind a disk. AIAA Journal, 46(5): 1118-1134, 2013/06/19 2008. doi: 10.2514/1.31074. URL http://arc.aiaa.org/ doi/abs/10.2514/1.31074.

O. Uzol, Y.-C. Chow, J. Katz, and C. Meneveau. Average passage flow field and deterministic stresses in the tip and hub regions of a multi-stage turbomachine. In ASME Turbo Expo 2003, collocated with the 2003 International Joint Power Generation Conference, pages 975-988. American Society of Mechanical Engineers, 2003.

F. B. Van Cleve and A. E. Copping. Offshore Wind Energy Permitting: A Survey of US Project Developers. Pacific Northwest National Laboratory, 2010.

C. VerHulst and C. Meneveau. Temporal characteristics of pod modes from wind farm les. Bulletin of the American Physical Society, 59, 2014. 
L. J. Vermeer, J. N. Sorensen, and A. Crespo. Wind turbine wake aerodynamics. Progress in Aerospace Sciences, 39:457-510, 2003.

W. Vision. A new era for wind power in the united states. Technical report, Technical report, US Department of Energy, Washington, DC, 2015.

J. Whale, K. Papadopoulos, C. G. Anderson, C. G. Helmis, and D. J. Skyner. A study of the near wake structure of a wind turbine comparing measurements from laboratory and full-scale experiments. Solar Energy, 56(6):621-633, 1996.

S. Wharton and J. K. Lundquist. Atmospheric stability affects wind turbine power collection. Environmental Research Letters, 7(1):014005, 2012.

Y.-T. Wu and F. Porté-Agel. Large-eddy simulation of wind-turbine wakes: evaluation of turbine parametrisations. Boundary-layer meteorology, 138(3):345-366, 2011. 


\section{Appendix A}

\section{Anisotropy of severely truncated bases}

The following development details the relationship of the POD mode basis to the invariants of the normalized Reynolds stress anisotropy tensor. According to the theory for the proper orthogonal decomposition, a low-order representation of the Reynolds stress tensor may be composed as the linear combination of POD modes and their respective eigenvalues,

$$
\begin{aligned}
\frac{\circ}{u_{i} u_{j}} N_{r} & =\sum_{n=1}^{N_{r}} \lambda^{(n)} \phi_{u_{i}}^{(n)} \phi_{u_{j}}^{(n)} \\
& =\left[\begin{array}{lll}
\sum_{n=1}^{N_{r}} \lambda^{(n)} \phi_{u}^{(n)} \phi_{u}^{(n)} & \sum_{n=1}^{N_{r}} \lambda^{(n)} \phi_{u}^{(n)} \phi_{v}^{(n)} & \sum_{n=1}^{N_{r}} \lambda^{(n)} \phi_{u}^{(n)} \phi_{w}^{(n)} \\
\sum_{n=1}^{N_{r}} \lambda^{(n)} \phi_{u}^{(n)} \phi_{u}^{(n)} & \sum_{n=1}^{N_{r}} \lambda^{(n)} \phi_{v}^{(n)} \phi_{v}^{(n)} & \sum_{n=1}^{N_{r}} \lambda^{(n)} \phi_{v}^{(n)} \phi_{w}^{(n)} \\
\sum_{n=1}^{N_{r}} \lambda^{(n)} \phi_{u}^{(n)} \phi_{w}^{(n)} & \sum_{n=1}^{N_{r}} \lambda^{(n)} \phi_{v}^{(n)} \phi_{w}^{(n)} & \sum_{n=1}^{N_{r}} \lambda^{(n)} \phi_{w}^{(n)} \phi_{w}^{(n)}
\end{array}\right] .
\end{aligned}
$$

The POD lends energy to the reconstructed stresses equally, rather than distributing energy following the balance of terms in the original stress tensor. This leads ultimately to an alteration of the anisotropic state of the turbulence as shown in the invariants of $\left.\stackrel{\circ}{b}_{i j}\right|_{N_{r}}$. Division by the turbulence kinetic energy is required to reach the normalized Reynolds stress anisotropy tensor. Following the low-order description above, the low-order tke is,

$$
\begin{aligned}
{\stackrel{\circ}{k_{N}}}_{N_{r}} & =\frac{1}{2} \operatorname{tr}\left({\frac{\circ}{u_{i} u_{j}}}_{N_{r}}\right) \\
& =\frac{1}{2} \sum_{n=1}^{N_{r}} \lambda^{(n)} \phi_{i}^{(n) 2} .
\end{aligned}
$$

\section{A.1 $N_{r}=1$}

Limiting the POD basis to a single mode results in the simple description of the turbulence kinetic energy as, 


$$
\stackrel{\circ}{k}_{1}=\frac{1}{2}\left(\lambda^{(1)} \phi_{u}^{(1) 2}+\lambda^{(1)} \phi_{v}^{(1) 2}+\lambda^{(1)} \phi_{w}^{(1) 2}\right) .
$$

The turbulence kinetic energy is used to normalize the low-order description of the Reynolds stress tensor.

$$
\stackrel{\circ}{b}_{i j, 1}=\left[\begin{array}{ccc}
\frac{\lambda^{(1)} \phi_{u}^{(1) 2}}{2 \grave{k}_{1}}-\frac{1}{3} & \frac{\lambda^{(1)} \phi_{u}^{(1)} \phi_{\nu}^{(1)}}{2 \grave{k}_{1}} & \frac{\lambda^{(1)} \phi_{u}^{(1)} \phi_{w}^{(1)}}{2 \grave{k}_{1}} \\
\frac{\lambda^{(1)} \phi_{u}^{(1)} \phi_{v}^{(1)}}{2 k_{1}} & \frac{\lambda^{(1)} \phi_{\nu}^{(1) 2}}{2 \dot{k}_{1}}-\frac{1}{3} & \frac{\lambda^{(1)} \phi_{v}^{(1)} \phi_{w}^{(1)}}{2 \dot{k}_{1}} \\
\frac{\lambda^{(1)} \phi_{u}^{(1)} \phi_{w}^{(1)}}{2 \grave{k}_{1}} & \frac{\lambda^{(1)} \phi_{v}^{(1)} \phi_{w}^{(1)}}{2 \grave{k}_{1}} & \frac{\lambda^{(1)} \phi_{w}^{(1)}}{2 \grave{k}_{1}}-\frac{1}{3}
\end{array}\right]
$$

Considering that only the first POD mode was used to formulate the above quantities, and that the large structures are organized to the beginning of the basis, $b_{i j, 1}$ is taken to represent only the most anisotropic turbulence.

As a consequence of normalization of the first invariant of $b_{i j}$, defined as the trace of the tensor is zero.

$$
\operatorname{tr}\left(\stackrel{\circ}{b}_{i j, 1}\right)=\frac{\lambda^{(1)}\left(\phi_{u}^{(1) 2}+\phi_{v}^{(1) 2}+\phi_{w}^{(1) 2}\right)-2 \stackrel{\circ}{k}_{1}}{2 \stackrel{\circ}{k}_{1}}=0
$$

However, the second and third invariants are non-zero quantities. In the low-order reconstructions shown here, the higher invariants take on special values not typically seen in real turbulence. The degree of anisotropy of the flow is well described by the second invariant of $b_{i j}$. For the low-order description using a single POD mode, the second invariant $\eta$ is equal to the trace of the square of the normalized Reynolds stress anisotropy tensor. With a single POD mode, $\eta$ is written,

$$
\stackrel{\circ}{\eta}_{1}=\frac{1}{6}\left(\frac{4 \stackrel{\circ}{k}_{1}^{2}-4 \stackrel{\circ}{k}_{1} \lambda^{(1)}\left(\phi_{u}^{(1) 2}+\phi_{v}^{(1) 2}+\phi_{w}^{(1) 2}\right)+3 \lambda^{(1) 2}\left(\phi_{u}^{(1) 2}+\phi_{v}^{(1) 2}+\phi_{w}^{(1) 2}\right)^{2}}{2 \stackrel{\circ}{k}_{1}^{2}}\right)^{1 / 2}
$$

Similarly, the third invariant of the normalized Reynolds stress anisotropy tensor, which describes the characteristic shape of the turbulence, is equated to the trace of the cube of normalized Reynolds stress anisotropy tensor. Using a single mode to describe the turbulence field, $\xi$ evolves as, 


$$
\begin{array}{r}
\stackrel{\circ}{\xi}_{1}=\frac{1}{6 \stackrel{\circ}{k}_{1}}\left(-4 \stackrel{\circ}{1}_{1}^{3}+6 \stackrel{\circ}{k}_{1}^{2} \lambda^{(1)}\left(\phi_{u}^{(1) 2}+\phi_{\nu}^{(1) 2}+\phi_{w}^{(1) 2}\right)-9 \stackrel{\circ}{1}_{1} \lambda^{(1) 2}\left(\phi_{u}^{(1) 2}+\phi_{v}^{(1) 2}+\phi_{w}^{(1) 2}\right)^{2}+\right. \\
\left.\frac{9}{2} \lambda^{(1) 3}\left(\phi_{u}^{(1) 2}+\phi_{\nu}^{(1) 2}+\phi_{w}^{(1) 2}\right)^{3}\right)^{1 / 3} .
\end{array}
$$

In the above definitions for $\stackrel{\circ}{1}_{1}$ and $\stackrel{\circ}{\xi}_{1}$, the expressions may be simplified by substitution of the reduced order turbulence kinetic energy $\stackrel{\circ}{k}_{1}$. In doing so, both invariants collapse identically to $\frac{1}{3}$ for the entire measurement domain. According to the Lumley triangle, $\eta=\xi=\frac{1}{3}$ corresponds to 1-component turbulence. Thus reduction of the POD mode basis to a single degree of freedom can represent only a single component of turbulence. The resultant turbulence need not be fixed to any coordinate system and in fact changes direction relative to the original measurements; its alignment in space is expressed by the corresponding eigenvectors of $b_{i j, 1}$.

\section{A.2 $N_{r}=2$}

Increasing the mode basis used in the low-order descriptions of the turbulence field to $N_{r}=2$ results in a similar but distinct development of the invariants of $b_{i j}$. With a basis of two POD modes, the low-order turbulence kinetic energy is written,

$$
\stackrel{\circ}{k}_{2}=\frac{1}{2}\left(\lambda^{(1)}\left(\phi_{u}^{(1) 2}+\phi_{v}^{(1) 2}+\phi_{w}^{(1) 2}\right)+\lambda^{(2)}\left(\phi_{u}^{(2) 2}+\phi_{v}^{(2) 2}+\phi_{w}^{(2) 2}\right)\right) .
$$

The two terms in equation (A.8) represent the respective contributions to the loworder tke by the first and second POD modes. Note that each normal stress is multiplied by the respective eigenvalue, indicating that energy is distributed evenly to the $u, v$, and $w$ components. The difference seen in the stresses is ultimately arbitrated by the POD modes rather than the eigenvalues. Using a basis of two mode, the normalized Reynolds anisotropy tensor is written,

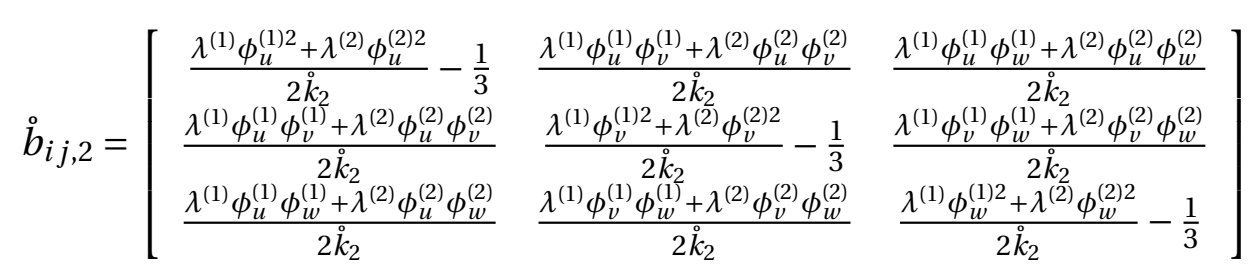

Normalization of $\left.\frac{0}{u_{i} u_{j}}\right|_{2}$ to arrive at $\stackrel{\circ}{b j, 2}_{i j}$ above is accomplished identically for that of $\left.\frac{0}{u_{i} u_{j}}\right|_{1}$. Consequently, the first invariant remains zero by definition. 


$$
\operatorname{tr}\left(\stackrel{\circ}{b}_{i j, 2}\right)=\frac{\lambda^{(1)}\left(\phi_{u}^{(1) 2}+\phi_{\nu}^{(1) 2}+\phi_{w}^{(1) 2}\right)+\lambda^{(2)}\left(\phi_{u}^{(2) 2}+\phi_{v}^{(2) 2}+\phi_{w}^{(2) 2}\right)-2 \stackrel{\circ}{k}_{2}}{2 \stackrel{\circ}{k}_{2}}=0 .
$$

Higher invariants quickly become quite complicated to write in full. The second and third invariants now include terms involving the squares of POD mode components $\left(\phi_{i}^{(n) 2}\right)$ as well as cross-rank mode products $\left(\phi_{i}^{(1)} \phi_{i}^{(2)}\right)$ and products of eigenvalues $\left(\lambda^{(1)} \lambda^{(2)}\right)$. The two-mode definition of $\eta$ is,

$$
\begin{aligned}
& \stackrel{\circ}{\eta}_{2}=\frac{1}{6 \dot{k}_{2}}\left(4 \stackrel{\circ}{k}_{2}^{2}-6 \stackrel{\circ}{k}_{2} \lambda^{(2)}\left(\phi_{v}^{(2) 2}+\phi_{w}^{(2) 2}\right)+3 \lambda^{(2) 2}\left(\phi_{v}^{(2) 2}+\phi_{w}^{(2) 2}\right)\left(\phi_{u}^{(2) 2}+\phi_{v}^{(2) 2}+\phi_{w}^{(2) 2}\right)+\right. \\
& 3 \lambda^{(1)} \lambda^{(2)}\left(-\phi_{u}^{(2) 2}\left(\phi_{v}^{(1) 2}+\phi_{w}^{(1) 2}\right)+2 \phi_{u}^{(1)} \phi_{u}^{(2)}\left(\phi_{v}^{(1)} \phi_{v}^{(2)}+\phi_{w}^{(1)} \phi_{w}^{(2)}\right)\right)+ \\
& \left.3 \lambda^{(1)} \lambda^{(2)}\left(\left(\phi_{v}^{(1)} \phi_{v}^{(2)}+\phi_{w}^{(1)} \phi_{w}^{(2)}\right)^{2}\right)\right)^{1 / 2} \text {. }
\end{aligned}
$$

Similarly, the two-mode definition of $\xi$ is,

$$
\begin{aligned}
& \stackrel{\circ}{\xi}_{2}=\frac{1}{6 \dot{k}_{2}^{2 / 3}}\left(8 \dot{k}_{2}^{2}-18 \stackrel{\circ}{k}_{2} \lambda^{(2)}\left(\phi_{v}^{(2) 2}+\phi_{w}^{(2) 2}\right)+9 \lambda^{(2) 2}\left(\phi_{v}^{(2) 2}+\phi_{w}^{(2) 2}\right)\left(\phi_{u}^{(2) 2}+\phi_{v}^{(2) 2}+\phi_{w}^{(2) 2}\right)\right. \\
& +9 \lambda^{(1)} \lambda^{(2)}\left(-\phi_{u}^{(2) 2}\left(\phi_{v}^{(1) 2}+\phi_{w}^{(1) 2}\right)+2 \phi_{u}^{(1)} \phi_{u}^{(2)}\left(\phi_{v}^{(1)} \phi_{v}^{(2)}+\phi_{w}^{(1)} \phi_{w}^{(2)}\right)\right) \\
& \left.+9 \lambda^{(1)} \lambda^{(2)}\left(\phi_{v}^{(1)} \phi_{v}^{(2)}+\phi_{w}^{(1)} \phi_{w}^{(2)}\right)^{2}\right)^{1 / 3} \text {. }
\end{aligned}
$$

While complicated in full, the two invariants can be simplified using the definition of the two-mode tke as done above for the single-mode approximation. Further, the invariants are related through an expression familiar to the analysis of turbulence anisotropy,

$$
\stackrel{\circ}{\eta}_{2}=\left(\frac{1}{27}+2 \stackrel{\circ}{\xi}_{2}^{3}\right)^{1 / 2}
$$

The relationship posed in equation (A.13) defines the upper boundary of the Lumley triangle and describes two-component turbulence. As for the expansion of the invariants with a single POD mode, the orientation of the two resultant components is well described by the eigenvectors of $\stackrel{\circ}{b}_{i j, 2}$.

The above development indicates that in order to reproduce three-dimensional turbulence, a minimum of three POD modes are required to formulate the truncated 
basis. Using fewer modes results in either one- or two-component turbulence fields. This is an intuitive result if one considers the POD modes to be degrees of freedom of a dynamical system. Although each POD mode vectorial in nature containing three distinct components, they represent a single projection of the fluctuating velocity fields and thus a single degree of freedom. In the development including three or more POD modes, the definitions of $\eta$ and $\xi$ become arduously long and have not been included here. 


\section{Appendix B}

\section{Galerkin projection of channel flow plane data}

Results in the following appendix pertain to the channel flow DNS performed at JHU (JHUCF). Sampled data is aligned with the mean flow direction as shown in Figure B.0.1. Data points are uniformly spaced in both the streamwise and wall-normal directions to ease numerical quadrature requirements. Regularly spaced data effects the ability to resolve near-wall turbulence, but it is not expected to effect the ability of the Galerkin system to simulate the time dependence of the modes. Parameters of the sampled data appear in Table B.1.

Table B.1: Details of $x y$-plane data sampled from channel flow DNS.

$\begin{array}{lcc}\text { spacing in } x \text {-direction } & \Delta x & 0.0079 \\ \text { spacing in } y \text {-direction } & \Delta y & 0.0079 \\ \text { time resolution between snapshots } & \Delta t & 0.0013 \\ \text { measurement points } & n_{x} \times n_{y} & 64 \times 64 \\ \text { number of snapshots } & n_{t} & 2048\end{array}$

A large number of snapshots were sampled from the total data in order to ensure statistical convergence of the turbulence field and the associated POD modes. The mean flow field for the sampled $x y$-plane is shown in Figure B.0.2. Turbulence as seen through the Reynolds stress tensor compared to the reconstructions resulting from POD and the dynamical system approximations below.

POD is applied to the channel flow data with a larger basis of snapshots $(N=2048)$ in order to guarantee convergence of POD modes. There are two distinct regions shown by the POD eigenvalues; a region of sharp decay for $n=1 . .250$, and a region where the eigenvalue spectrum is significantly more uniform. Example POD modes used in calculating the GS and following LODS are shown in Figure B.0.4.

Figures B.0.6 and B.0.7 demonstrate the parameters resulting from projection of the governing behavior law onto the POD basis truncated to 6 out of 2048 modes, representing approximately $70 \%$ of the turbulence kinetic energy. The quadratic parameter accounting for the projection of the POD basis onto the pressure term of the NS equations has been omitted as it is three orders of magnitude smaller than the convection 


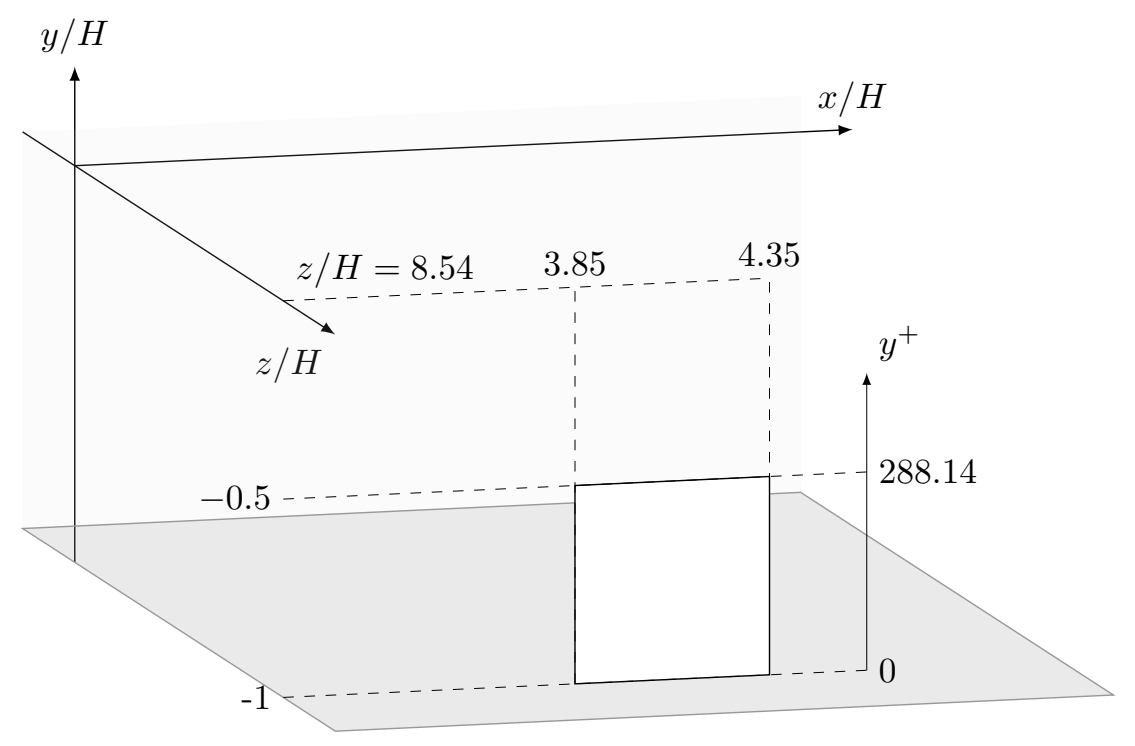

Figure B.0.1: Schematic of the sampled channel flow in an $x y$-plane for the development of a Galerkin and low-order dynamical systems.
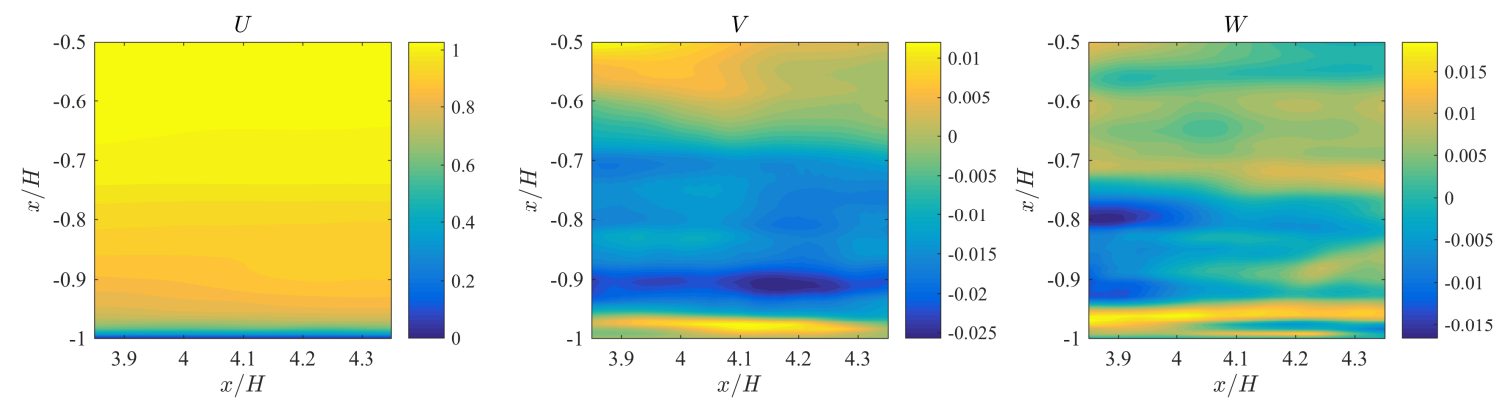

Figure B.0.2: Mean velocities in the streamwise, wall-normal, and spanwise (out of plane) directions. Mean velocity field are taken as the 'zeroth' POD mode in the GS, $\Phi^{(0)}$.

parameter. Comparing the magnitudes of the viscous and convection parameters in Figures B.0.6, B.7(A), and B.7(B), one notes that the viscous term appears to be 500 - 1000 times larger. This difference is accounted for in product with the dimensionless viscosity $v=5 \times 10^{-3}=1 / \mathrm{Re}$. The linear parameter of the GS exaggerates selfinteraction of the mode basis, shown in the figure along the diagonal.

The quadratic parameter, here accounting for convection (Figure B.7(A)) and production (Figure B.7(B)), excludes the pressure contribution discussed above. The three-dimensional matrix of parameters is roughly symmetric about $i=j$ and anti- 


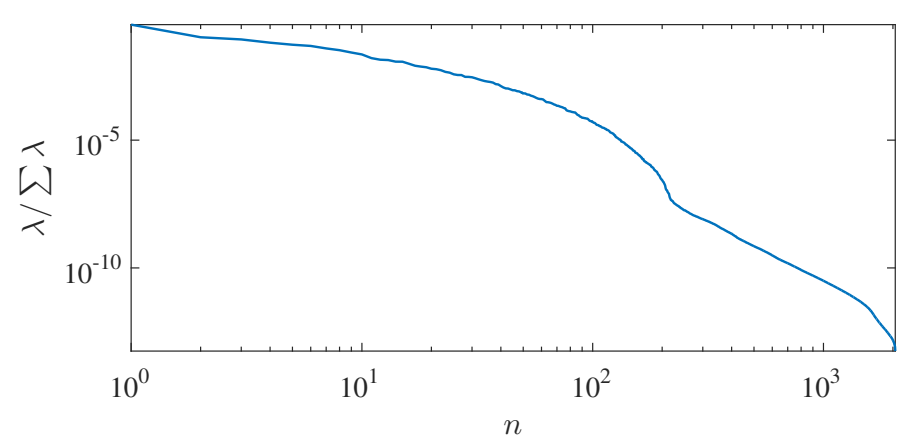

Figure B.0.3: POD eigenvalues for plane data.

symmetric about $i=k$. The resulting dynamic system is quite sensitive to the numerical values of the parameters. The leftmost column of each of the parameters in Figure B.0.6 and B.0.7 represent the interaction of the mean flow ('zeroth mode') with the turbulence field. In all shown cases, the mean flow/turbulence interactions designated by $L_{i 0}, q_{i 01}$, and $q_{i 10}$ are nearly null, which signifies that the stationary mean flow is not necessary to the dynamics of the GS.

Figure B.0.8 compares the coefficients resulting from projecting the modes onto the basis of snapshots ( $a_{i}$, solid lines) to those resulting from projecting the modes onto the governing equations ( $\hat{a}_{i}$, dashed lines). It is evident from the figure that the Galerkin system does not sufficiently account for the variation seen in the mode coefficients derived directly from POD even within the original input time range. The limited variability of the coefficients arises from the definitions of the parameters $l_{i j}$ and $q_{i j k}$. Effects of numerical dissipation are increased, seen as damping in the coefficients of the Galerkin system seen in Figure B.0.8.

Artificial damping of the system can be mitigated by introducing intermediate modes to the GS parameters, although the particular effects are necessarily a function of the particular selection of modes introduced to the system. To illustrate the difference in the behavior, intermediate modes are introduced and the GS parameters re-calculated. For consistency, a total of six modes are used in each of the POD mode bases used in the following modified Galerkin systems. Figure B.0.9 shows the evolution of the dynamical system using a variety of mode combinations. The particular modes used in each of the GS modifications were selected to show the range of effects associated with energetic, intermediate, and isotropic POD modes.

In each of the cases shown in Figure B.0.9 the long-term behavior of the GS is quite different and demonstrates the capability of the GS to make predictions of the channel flow velocity field. The original GS becomes absolutely stable beyond the input time due to numerical damping. This effect is an obvious artifact of the numerical process rather than a balance present in the system or the flow. The modified GS do not have 

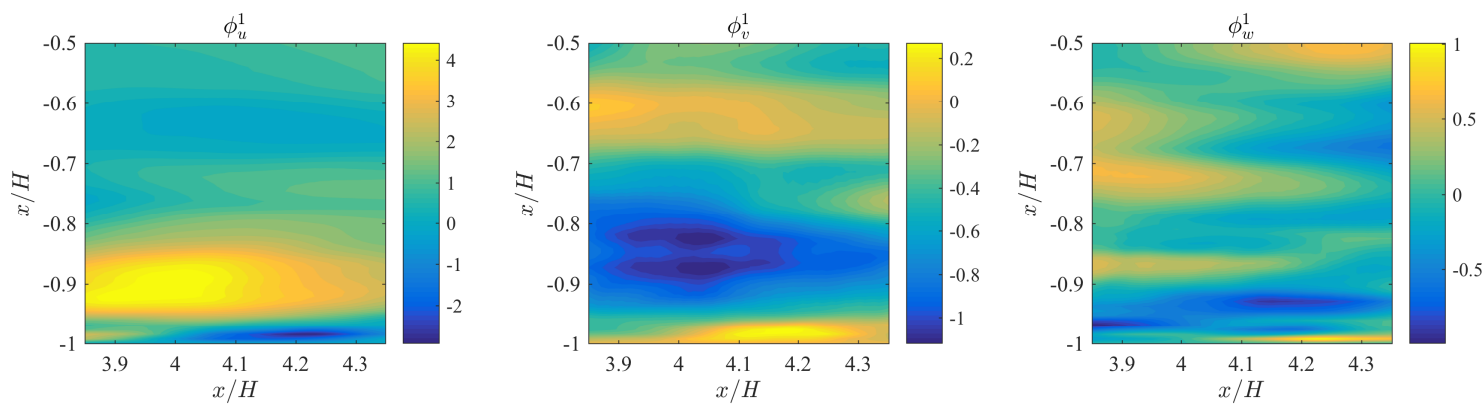

(A) $\Phi^{(1)}$
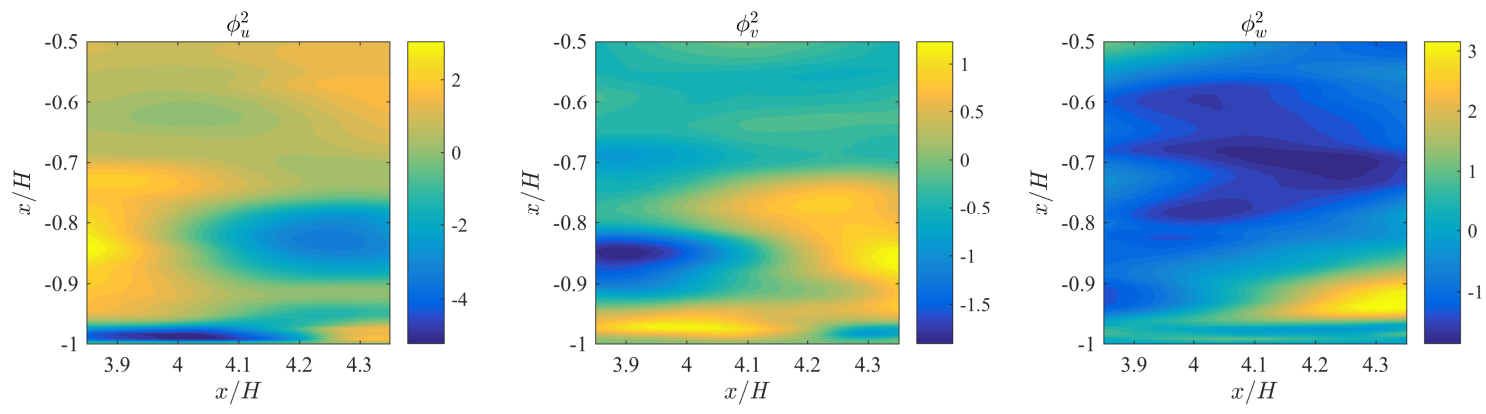

(B) $\boldsymbol{\Phi}^{(2)}$
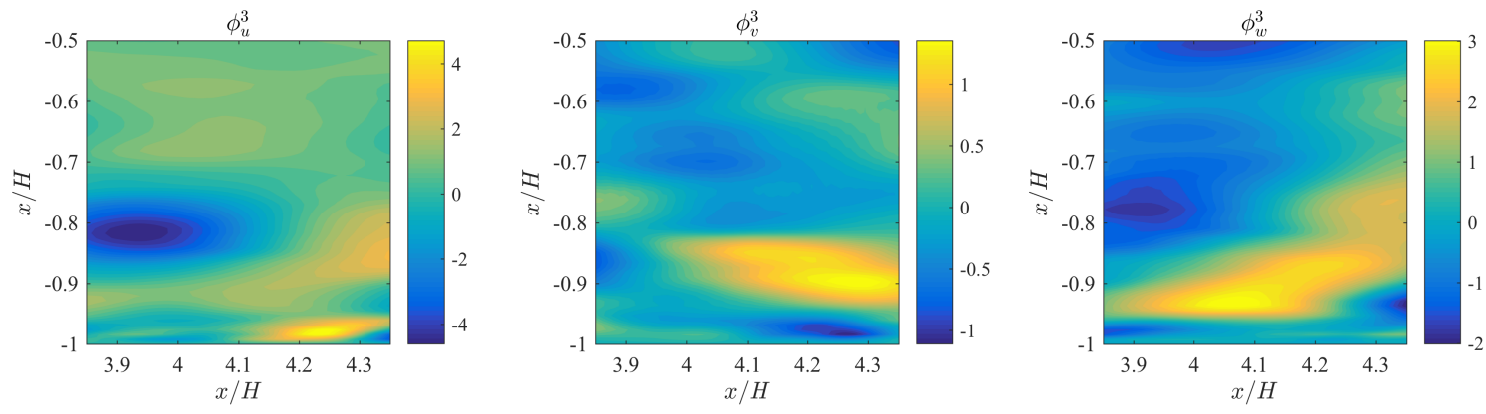

(C) $\boldsymbol{\Phi}^{(3)}$

Figure B.0.4: POD modes 1 - 3. From left are the streamwise $\left(\phi_{u}^{(i)}\right)$, wall-normal $\left(\phi_{v}^{(i)}\right)$, and spanwise $\left(\phi_{w}^{(i)}\right)$ components of each mode.

long-term behavior that can be predicted $a$ priori. As an illustration, the second modification of the GS (Figure A.B.9(A)) is more stable than the original system despite using non-consecutive modes, including some that are in the intermediate range. Mode bases that include only $\boldsymbol{\Phi}^{(1)}$ and $\boldsymbol{\Phi}^{(2)}$ from the energetic mode range result in GS that show significantly more complex behavior. This is a possible path for further exploration, but there is not an obvious choice of the POD modes to include in the 'ideal' 

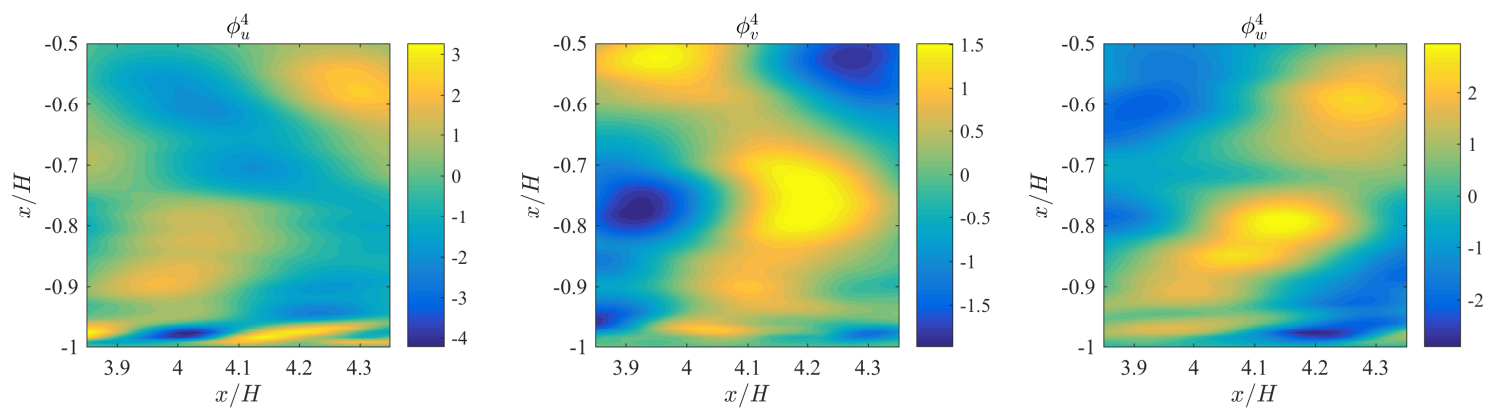

(A) $\boldsymbol{\Phi}^{(4)}$
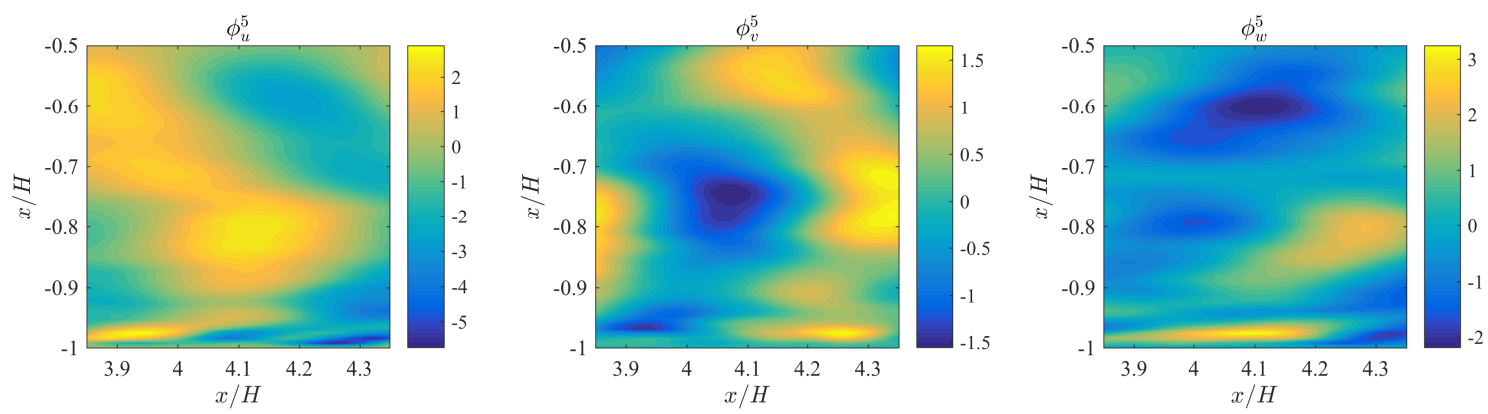

(B) $\boldsymbol{\Phi}^{(5)}$
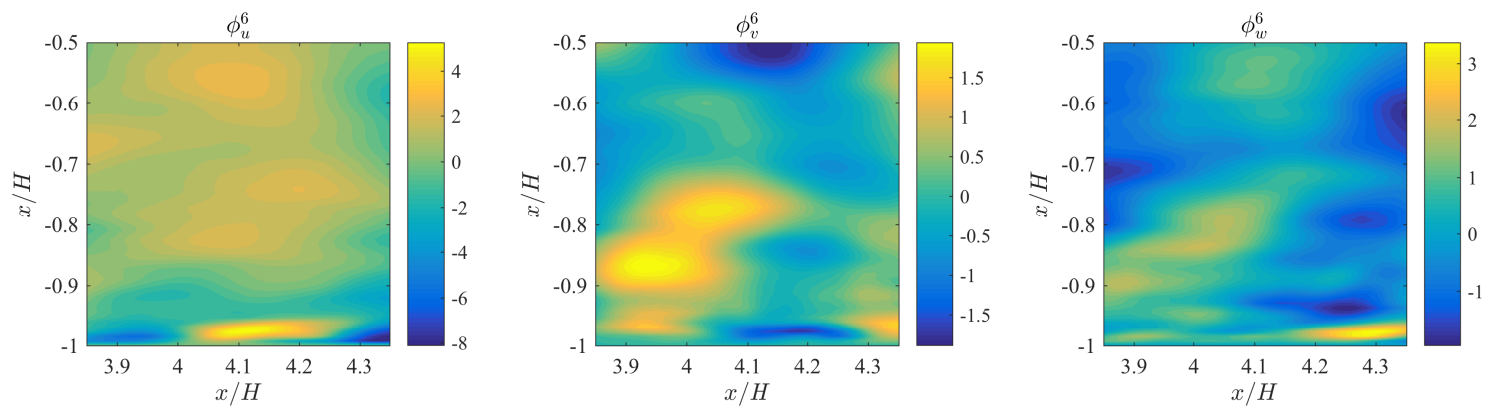

(C) $\boldsymbol{\Phi}^{(6)}$

Figure B.0.5: POD modes 4 - 6. From left are the streamwise $\left(\phi_{u}^{(i)}\right)$, wall-normal $\left(\phi_{v}^{(i)}\right)$, and spanwise $\left(\phi_{w}^{(i)}\right)$ components of each mode.

GS. 


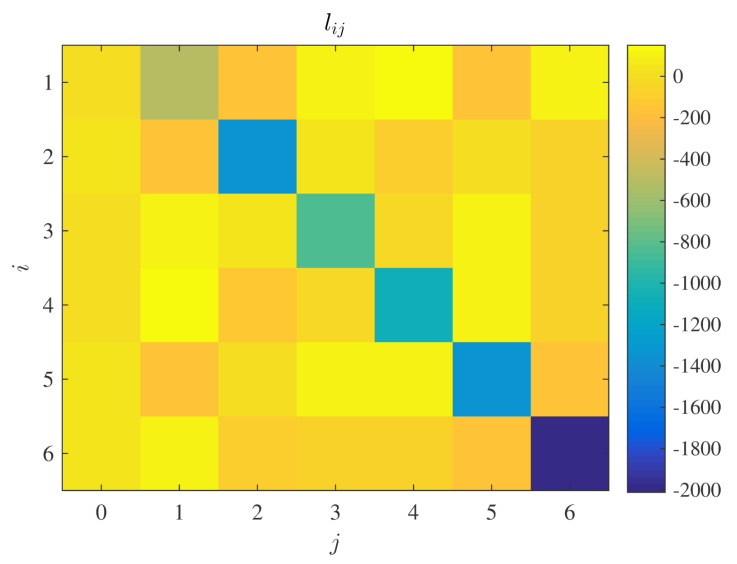

Figure B.0.6: Linear term from dynamical system. $l_{i j}$ results from projecting the POD basis onto the viscous dissipation term of the Navier-Stokes equations.

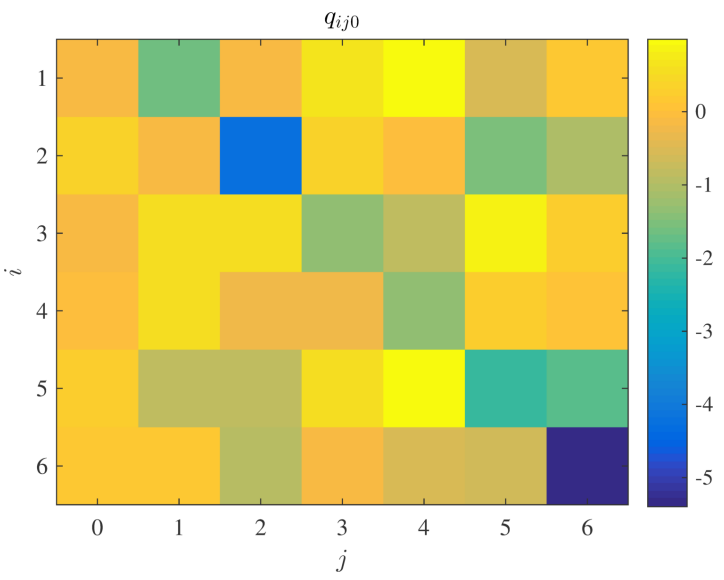

(A)

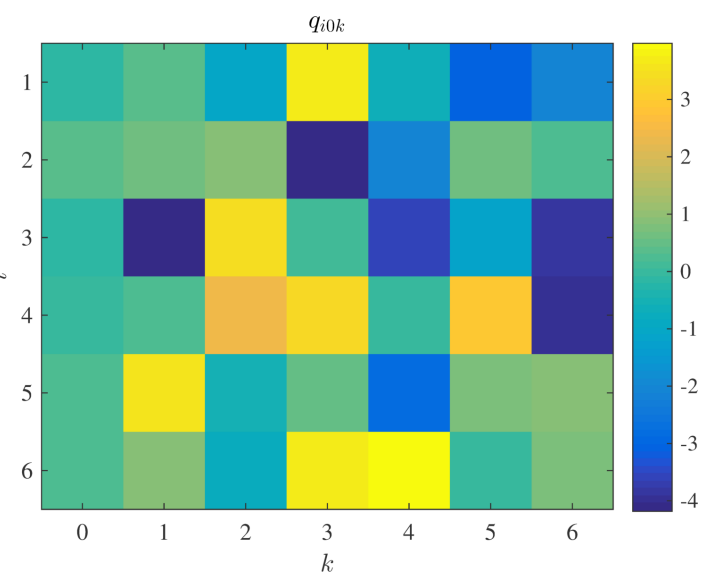

(B)

Figure B.0.7: Quadratic term from dynamical system. $q_{i j k}$ results from projecting the POD basis onto the convection term of the Navier-Stokes equations. Fixing index $k$ to 0 limits the parameter to the description of convection in the GS. Fixing index $j$ to 0 limits the parameter to the description of production in the GS. 


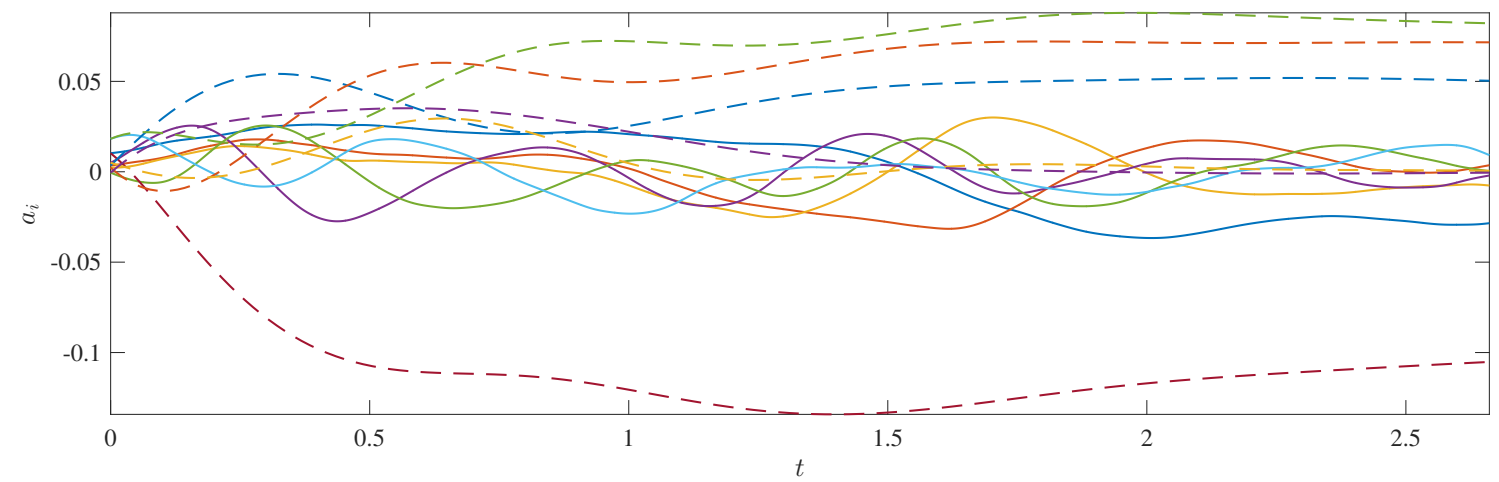

Figure B.0.8: POD mode coefficients (solid lines) compared to the coefficients arising from solution of the GS (dashed lines). 


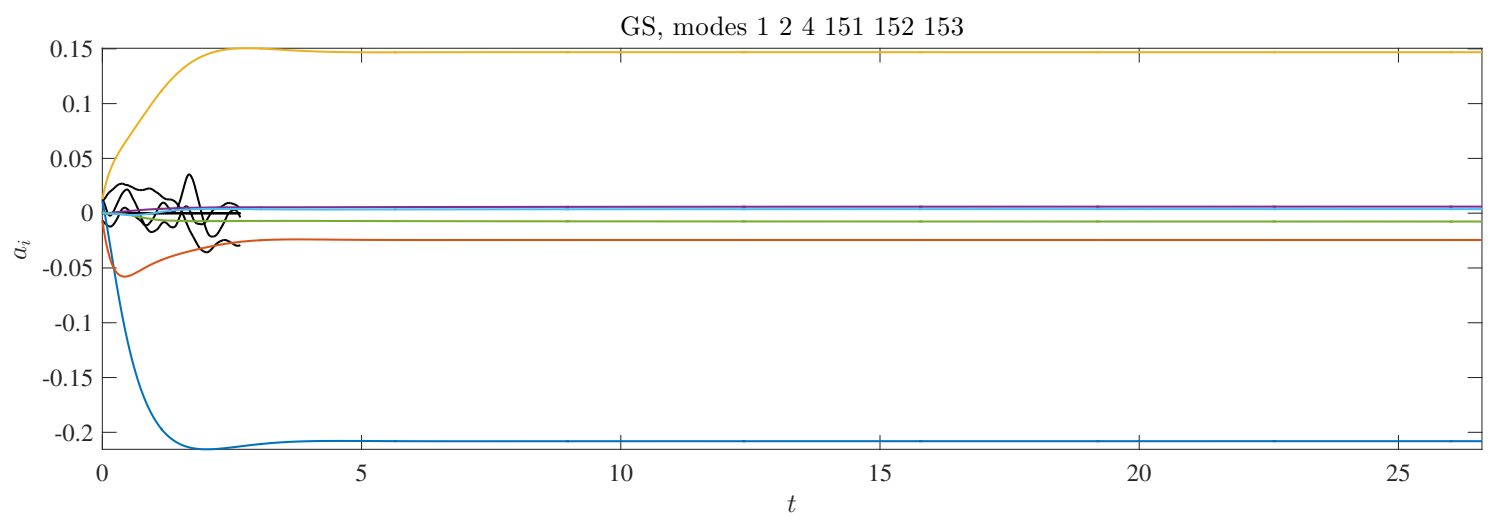

(A) $\operatorname{Mod} 2$

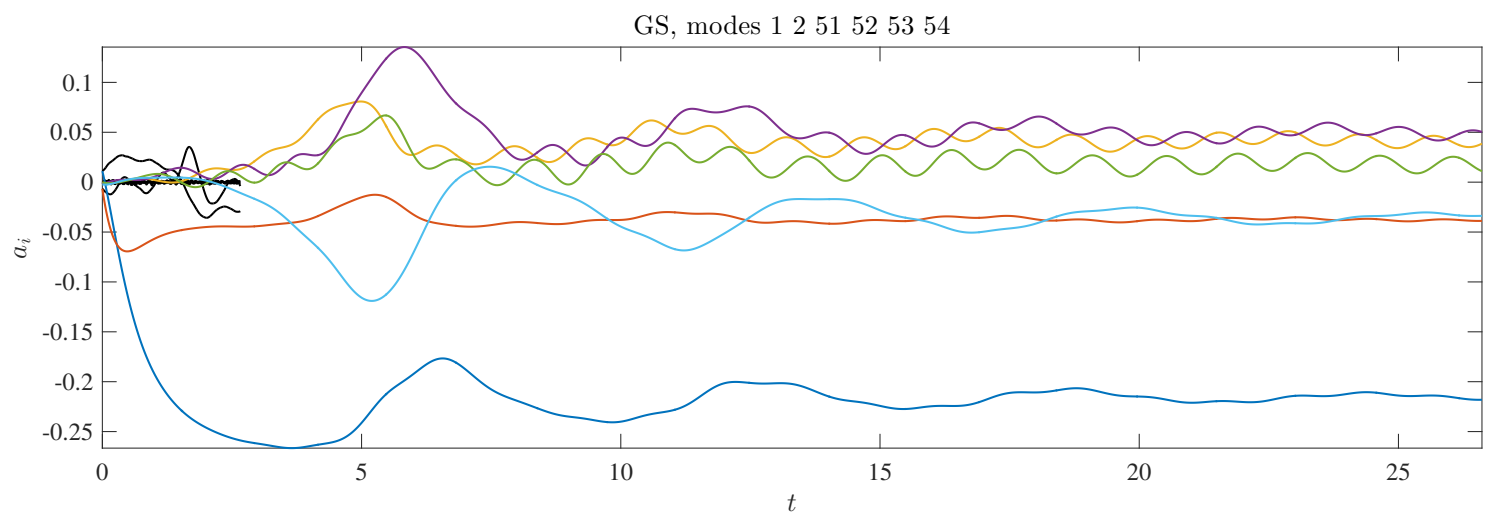

(B) $\operatorname{Mod} 3$

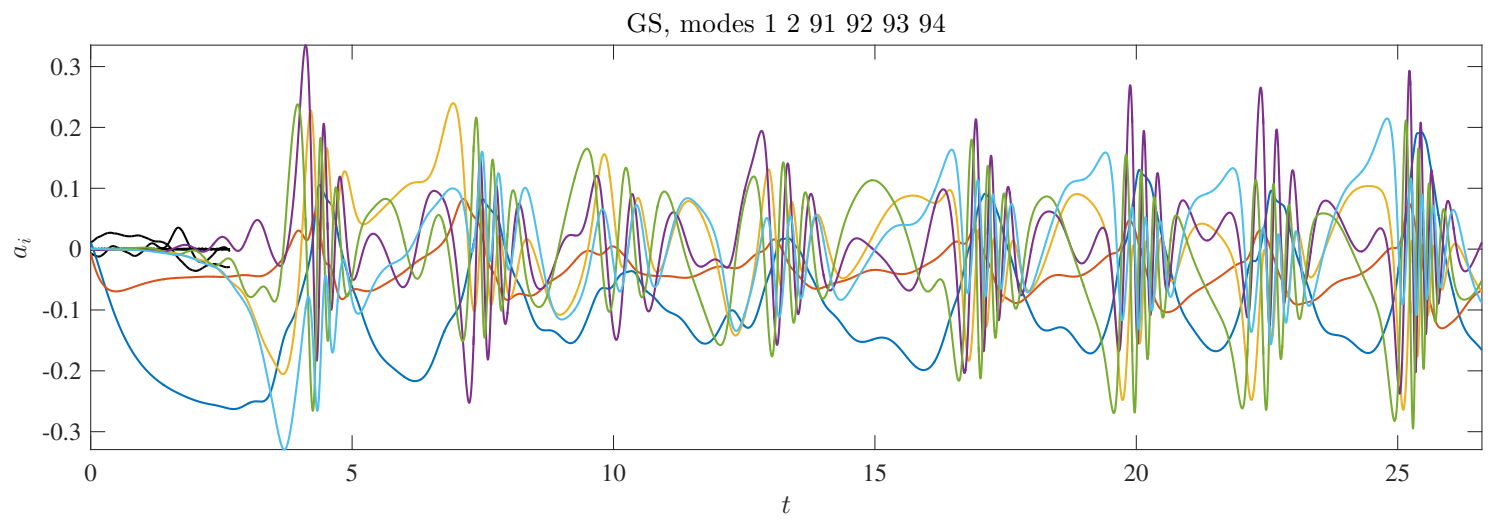

(C) $\operatorname{Mod} 4$

Figure B.0.9: GS modifications to illustrate long term behavior and sensitivity to input mode basis. 


\section{Appendix C}

\section{DPOD LODS}

In the present application, $x z$-planes of data are extracted from the volume discussed above. The POD is then applied iteratively to each plane, with increasing wall-normal coordinate $y / H$. The wall normal direction was selected as the decomposition dimension as the others are expected to exhibit homogenous behavior. The simulation focuses on a fully developed flow, meaning that any gradient in the streamwise direction is expected to be quite small compared to those in the wall normal direction. The imposed periodicity in the spanwise direction of the simulation results in homogeneous statistics along $z$. Applying the proper orthogonal decomposition to homogeneous data results in modes that closely (or exactly) resemble Fourier modes. When this is the case, the POD offers no improvement in the optimality of the basis to represent the input data, but does require additional computation. For these reasons, the only direction that remains feasible for application of the DPOD is the wall-normal coordinate. The output then describes the evolution of POD modes $\Phi^{(n)}(x, z)$ vertically, perpendicular to the channel wall.

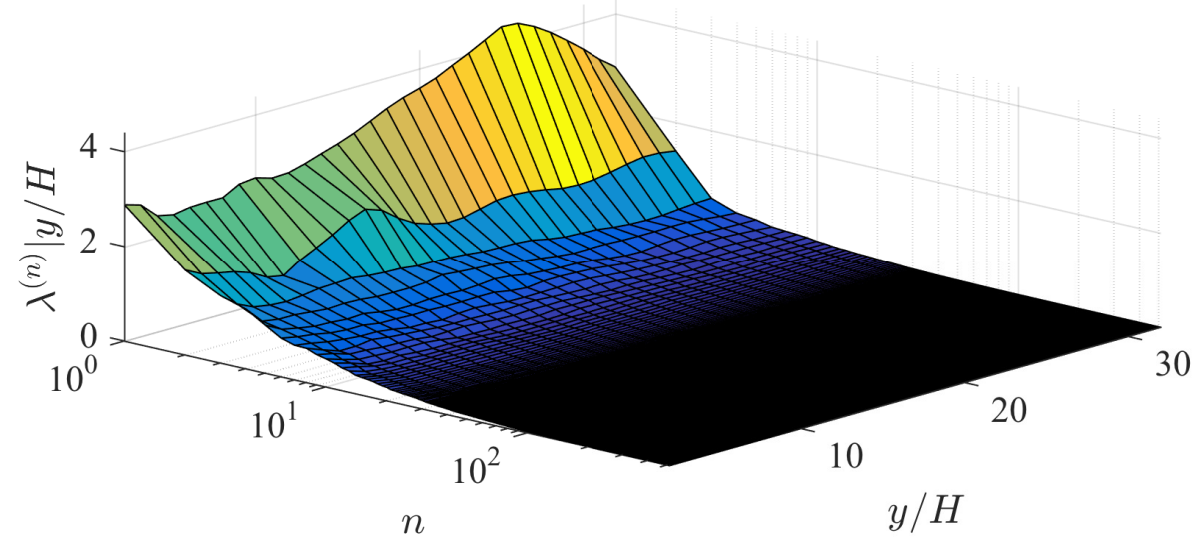

Figure C.0.1: POD eigenvalues by $x z$-plane. 


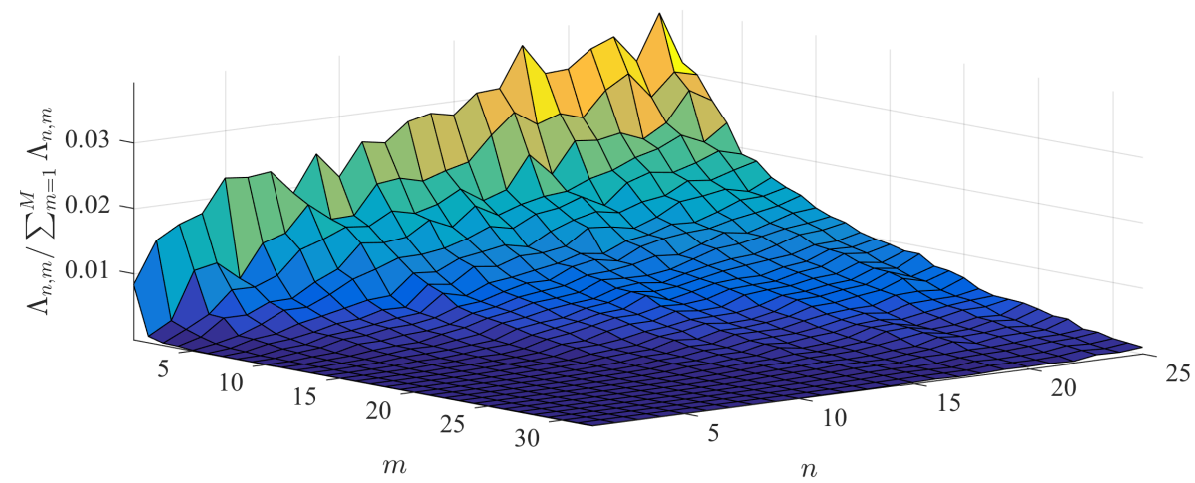

Figure C.0.2: DPOD eigenvalues $\Lambda_{n}, m$ normalized by the sum over sub-modes.

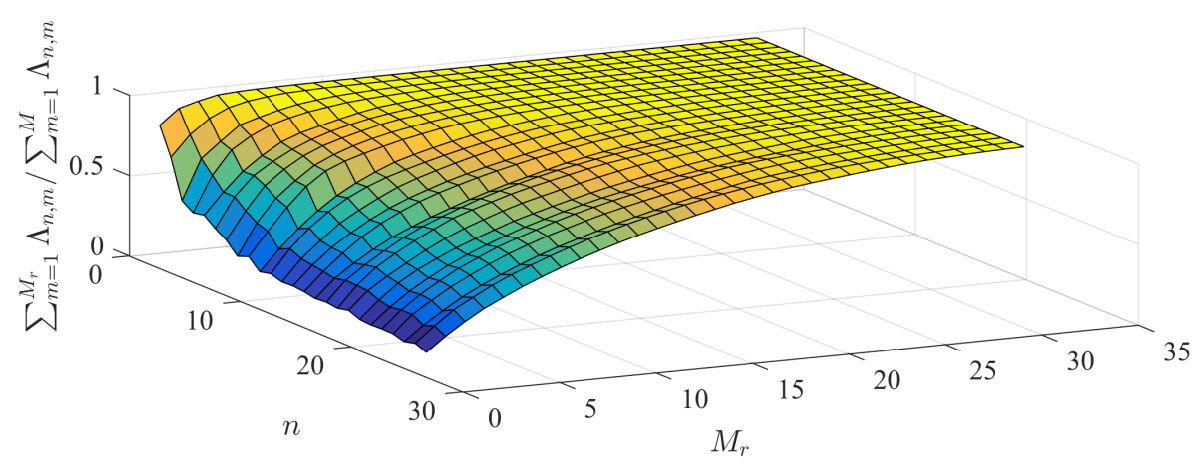

Figure C.0.3: Cumulative summation of $\Lambda_{n, m}$ by POD mode.

\section{C.1 Least squares polynomial fit}



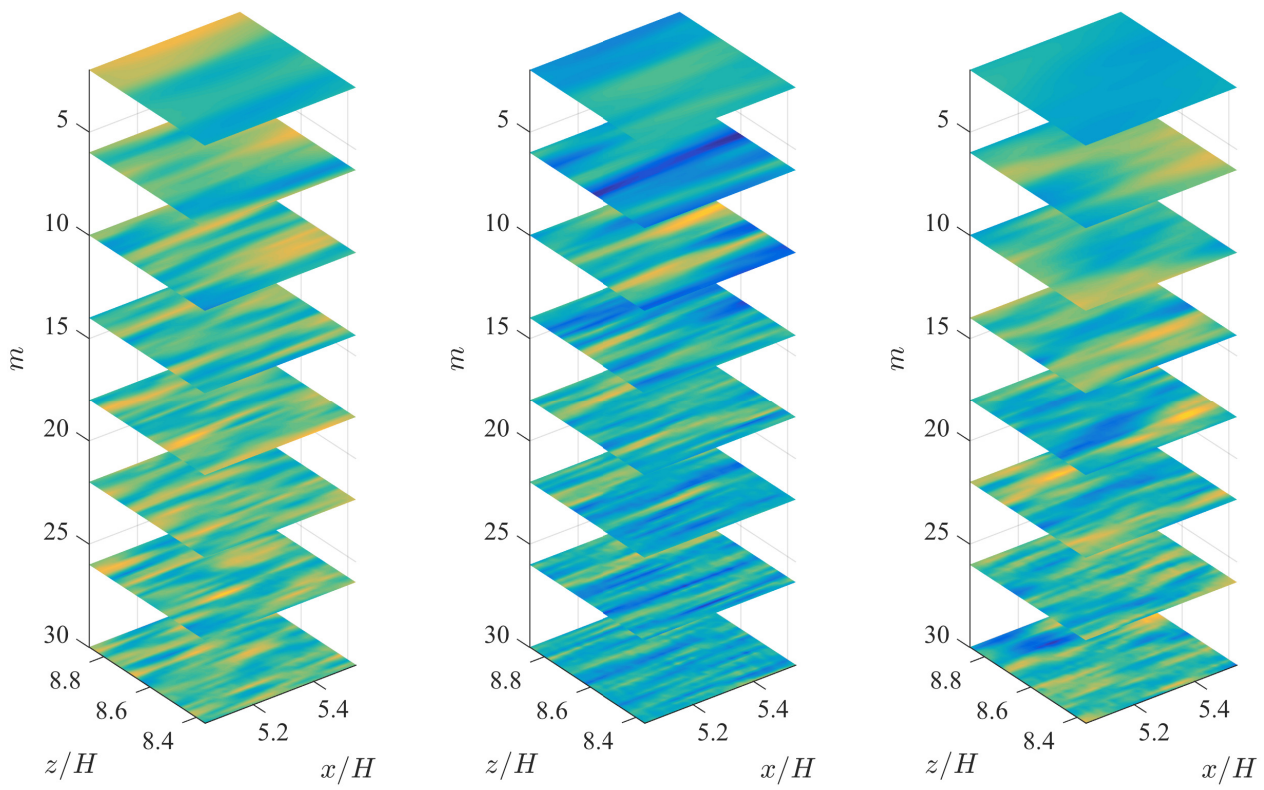

Figure C.0.4: $\Gamma^{(1, m)}$. Streamwise, wall-normal, and spanwise components from left. Every fourth submode shown for clarity.
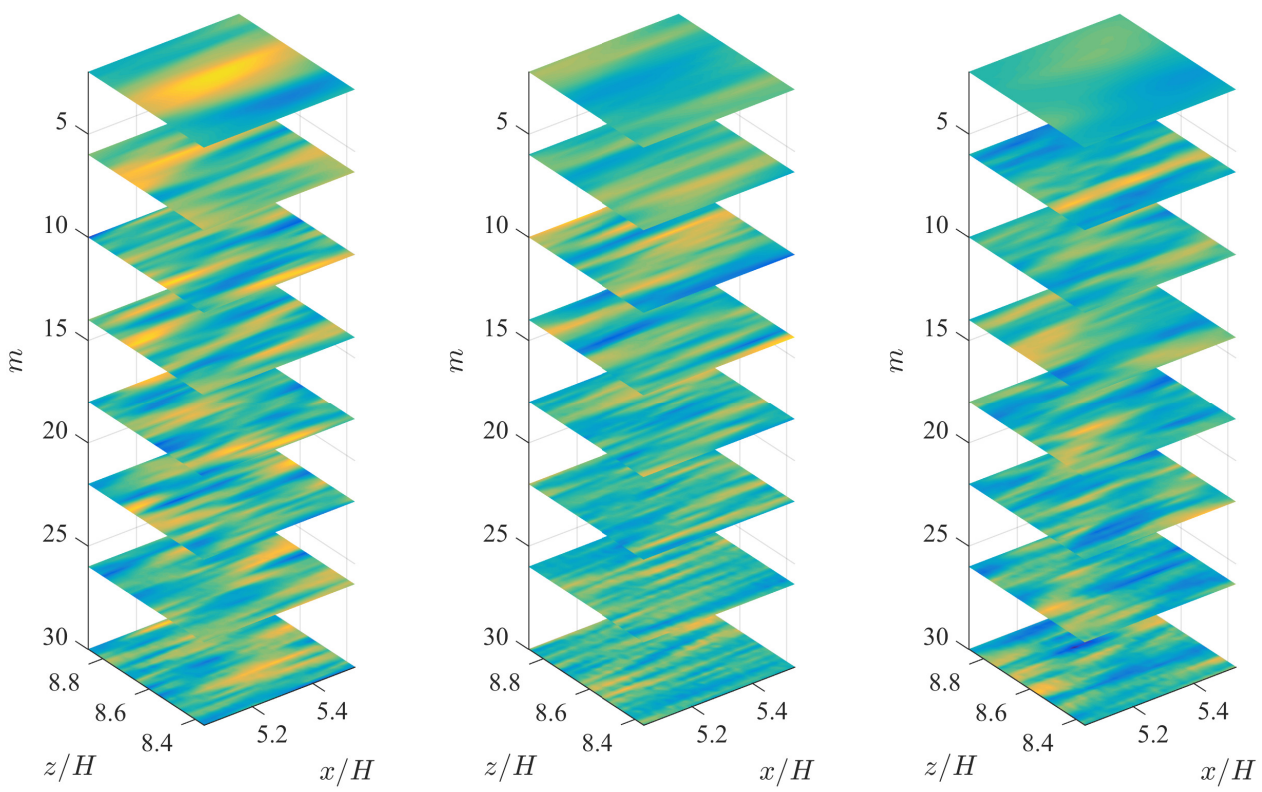

Figure C.0.5: $\Gamma^{(2, m)}$. Streamwise, wall-normal, and spanwise components from left. Every fourth submode shown for clarity. 

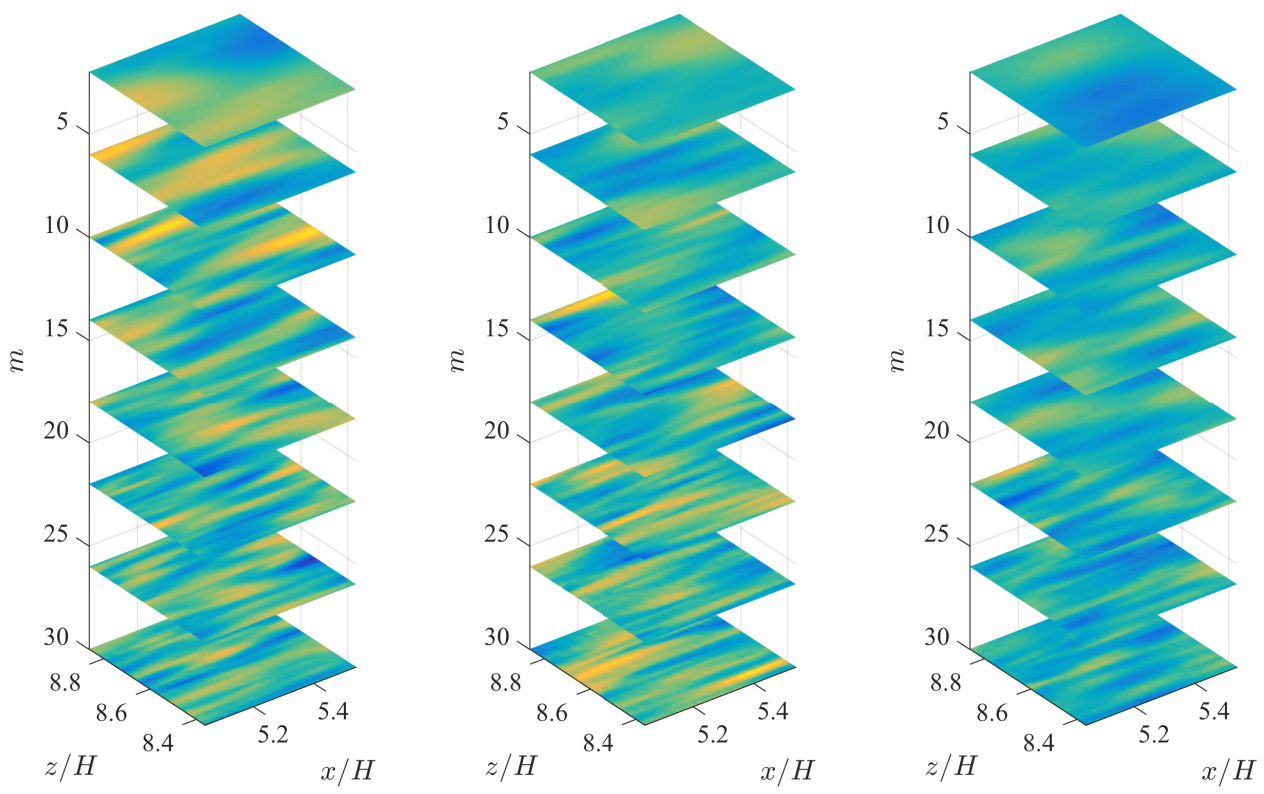

Figure C.0.6: $\Gamma^{(3, m)}$. Streamwise, wall-normal, and spanwise components from left. Every fourth submode shown for clarity.
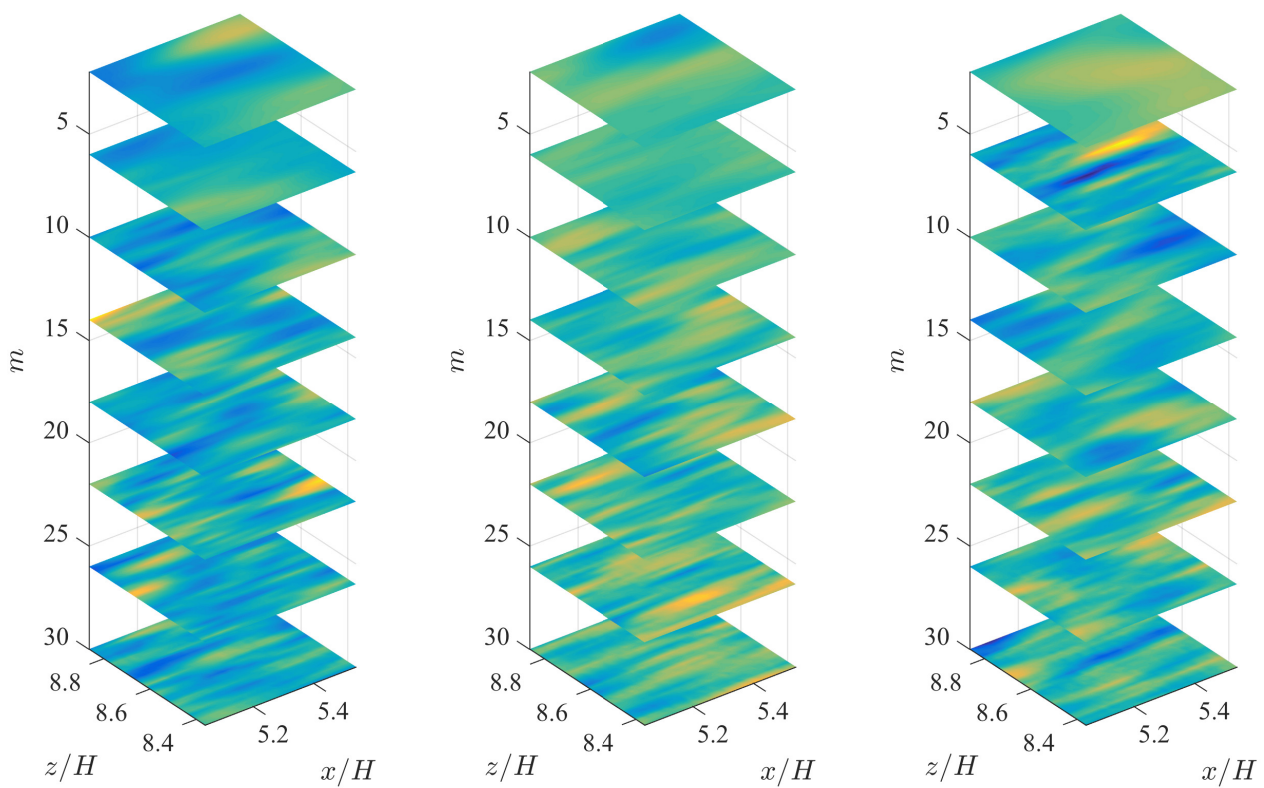

Figure C.0.7: $\Gamma^{(4, m)}$. Streamwise, wall-normal, and spanwise components from left. Every fourth submode shown for clarity. 


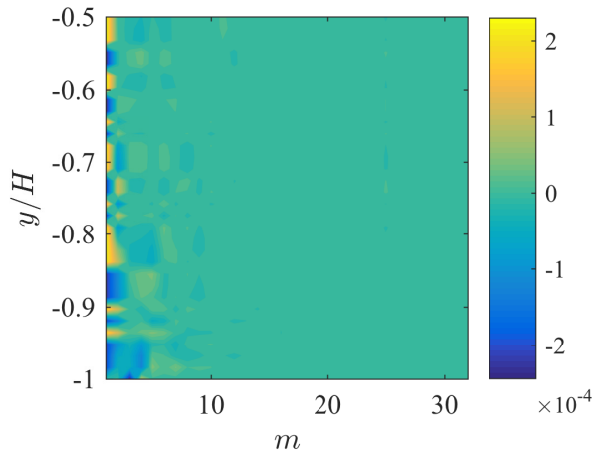

(A) DPOD coefficient $b_{1, m}(y / H)$.

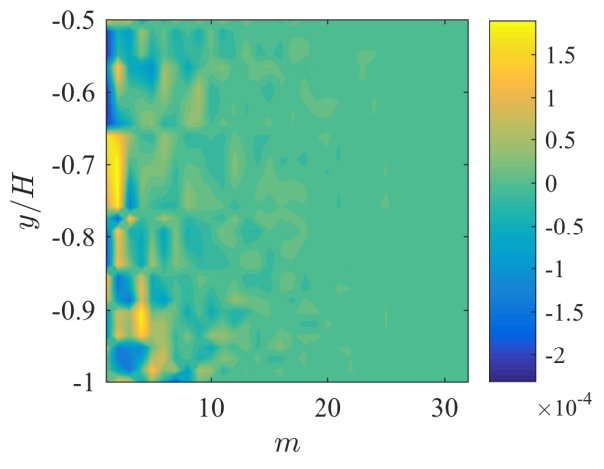

(C) DPOD coefficient $b_{3, m}(y / H)$.

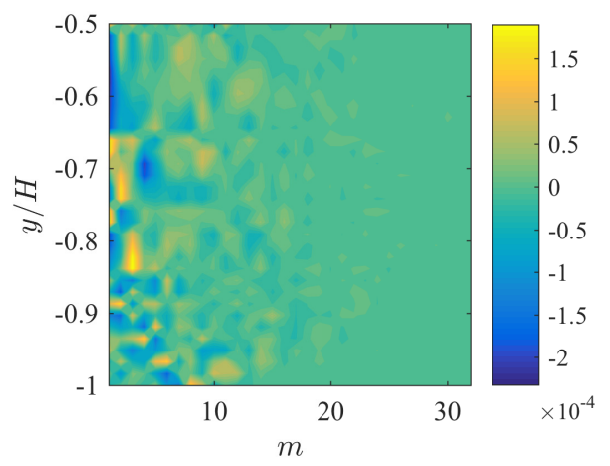

(E) DPOD coefficient $b_{5, m}(y / H)$.

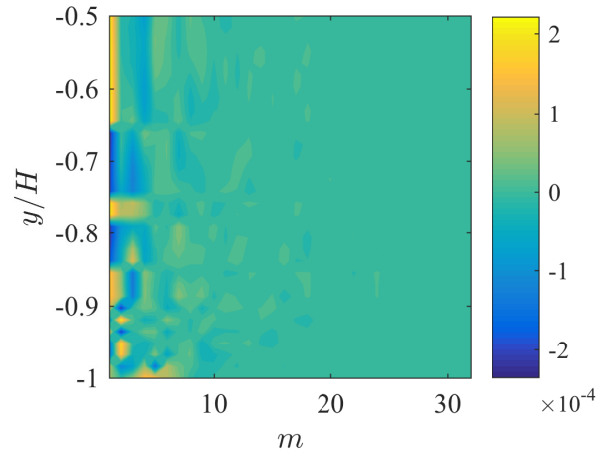

(B) DPOD coefficient $b_{2, m}(y / H)$.

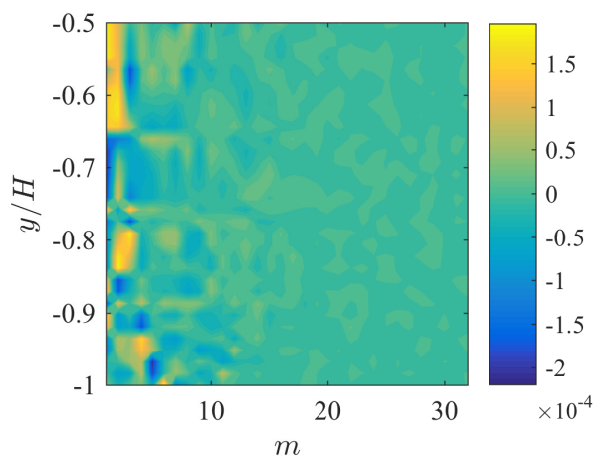

(D) DPOD coefficient $b_{4, m}(y / H)$.

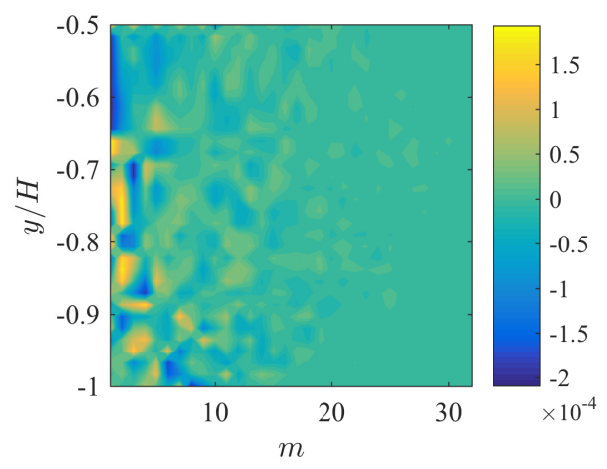

(F) DPOD coefficient $b_{6, m}(y / H)$.

Figure C.0.8: Submode coefficients for the first 5 POD mode bases in the channel flow. Each coefficient spans the submodal basis $(m)$ and the wall-normal coordinate according to the formulation $(y / H)$. 
The dynamical system is now formed by estimating the spatial evolution of the submodal coefficients $b_{n, m}$ according to the coordinate $y$. Note that each low-order dynamical system defined below (indexed with $n$ ) pertains to the wall-normal evolution of a single POD mode. In order to make predictions of a basis of POD modes $(\hat{\Phi})$, the LODS must be posed and solved iteratively.

$$
\frac{d b_{n, i}}{d t}=D_{i}+\sum_{j=1}^{M} L_{i j} b_{n, j}+\sum_{j, k=1}^{M} Q_{i j k} b_{n, j} b_{n, k}+\sum_{j, k, l=1}^{M} C_{i j k l} b_{n, j} b_{n, k} b_{n, l}
$$

In equation (C.1), the parameters no longer arise from projecting the governing behavior law onto the DPOD basis but rather from a least squares fit of monomial terms onto the coefficients directly. The parameters sought are $D_{i}, L_{i j}, Q_{i j k}$, and $C_{i j k l}$, standing for constant, linear, quadratic, and Cubic contributions, respectively. Parameters are calculated iteratively for each input mode $i$ and involve many terms. Each index $i, j, k, l$ spans the DPOD basis selected (i.e. $M_{r}$ out of $M$ sub-modes).

A more consolidated way to write the dynamical system is,

$$
\frac{d b_{n, i}}{d t}=\sum_{k=1}^{M} x_{k} b_{n, k}\left(b_{n, 1}, \ldots, b_{n, M}\right)
$$

where $x_{k}$ are the unknown parameters and $b_{n, k}$ are the monomial terms at most cubic. Parameters are found by minimizing the error function,

$$
\chi^{2}=\sum_{p=1}^{M}\left[\frac{d b_{n, i}}{d t}-\sum_{k=1}^{M} x_{k} b_{n, k}\left(b_{n, 1}\left(y_{p}\right), \ldots, b_{n, M}\left(y_{p}\right)\right)\right]^{2}
$$

e.g. $\chi^{2}=|A X-B|^{2}$ where $X$ is the vector of unknown coefficients, $B$ the vector containing the $M$ values of $\frac{d b_{n, i}}{d t}$ and $A$ the matrix of terms $b_{n, j}\left(t_{p}\right), b_{n, j}\left(t_{p}\right) b_{n, k}\left(t_{p}\right)$, and $b_{n, j}\left(t_{p}\right) b_{n, k}\left(t_{p}\right) b_{n, l}\left(t_{p}\right)$.

\section{C.2 reconstructions and error calcs}



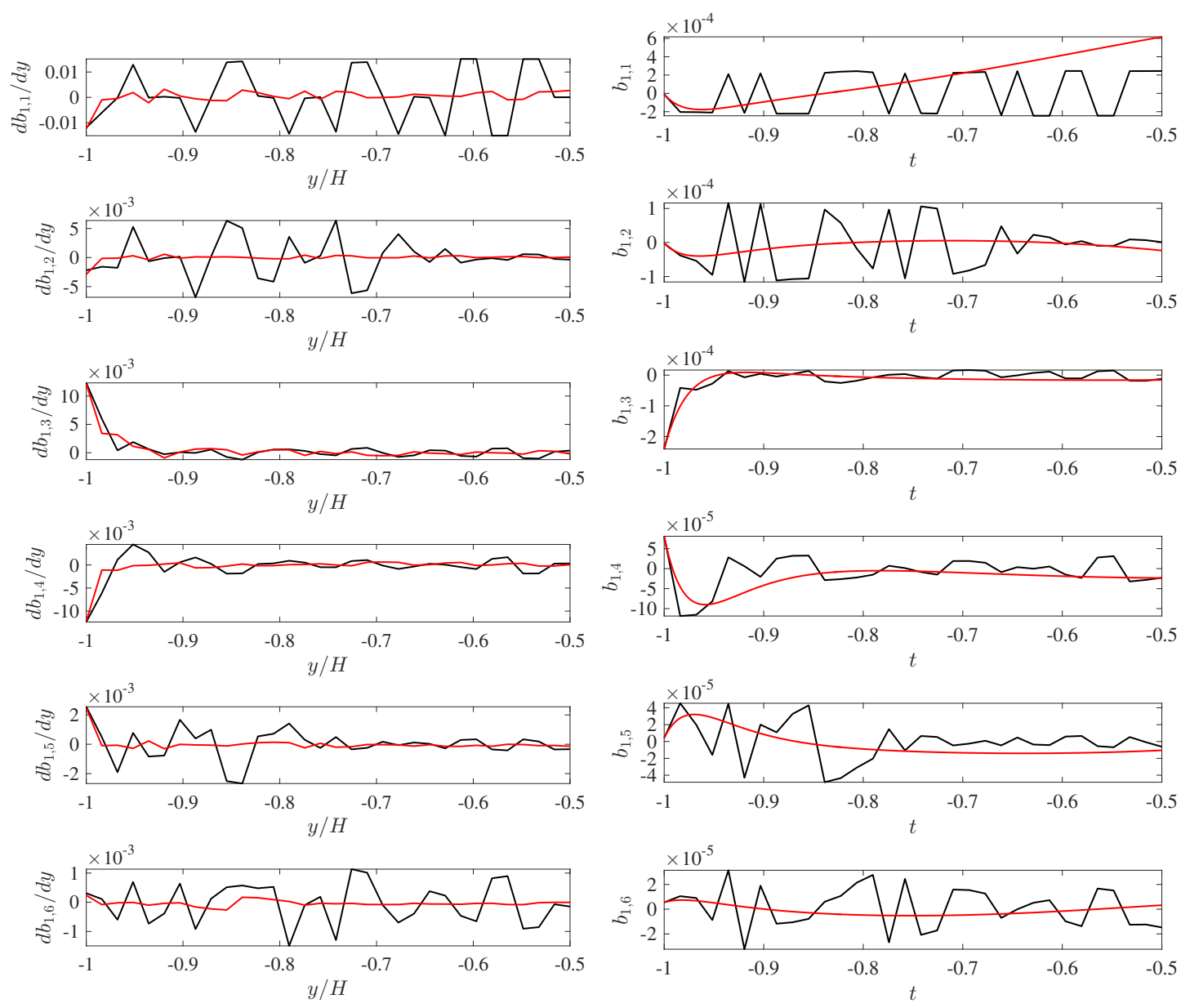

(A) $d \hat{b}_{1, m} / d y$

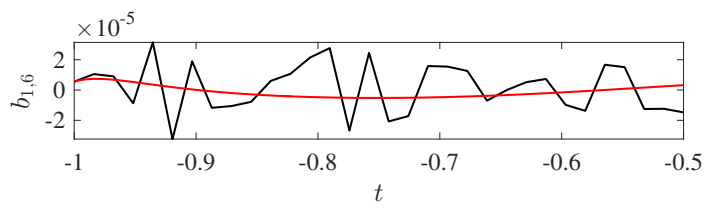

(B) $\hat{b}_{1, m}$

Figure C.1.9: LODS predictions for $\hat{b}_{1, m}$ (right), based on the parameter fit of $d \hat{b}_{1, m} / d y$ (left). 

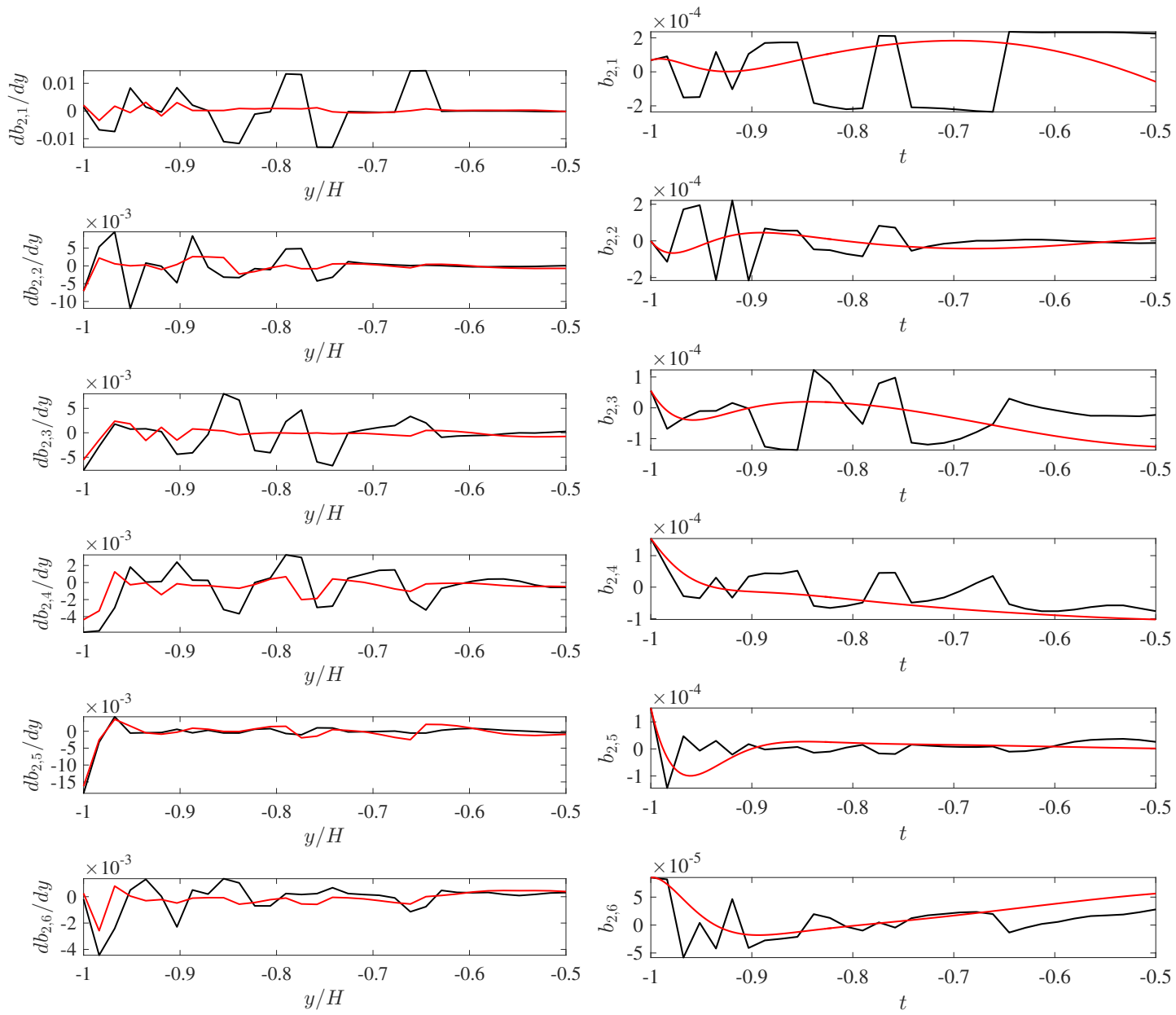

(A) $d \hat{b}_{2, m} / d y$

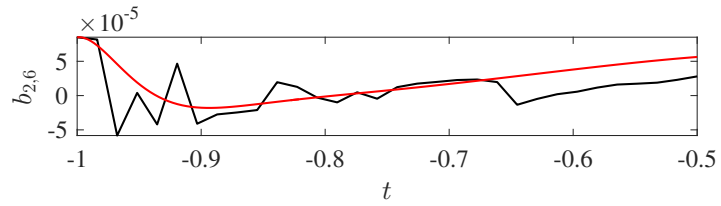

(B) $\hat{b}_{2, m}$

Figure C.1.10: LODS predictions for $\hat{b}_{2, m}$ (right), based on the parameter fit of $d \hat{b}_{2, m} / d y$ (left). 

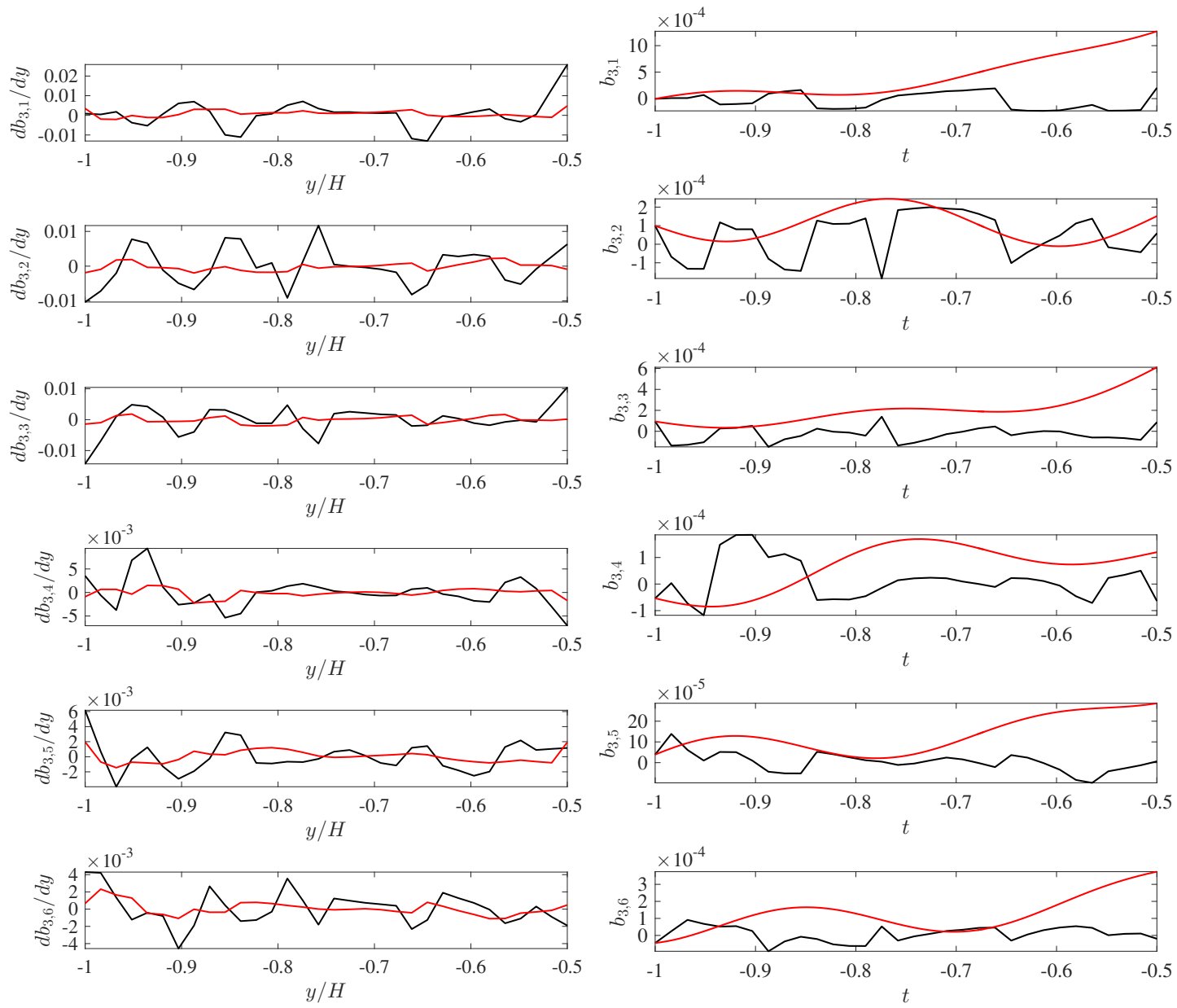

(A) $d \hat{b}_{3, m} / d y$

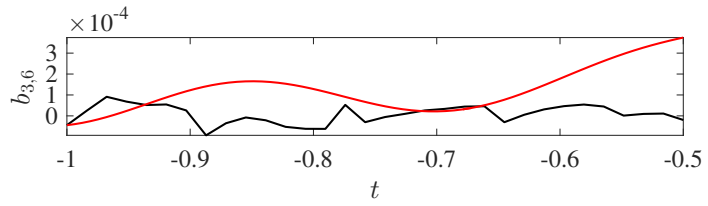

(B) $\hat{b}_{3, m}$

Figure C.1.11: LODS predictions for $\hat{b}_{3, m}$ (right), based on the parameter fit of $d \hat{b}_{3, m} / d y$ (left). 

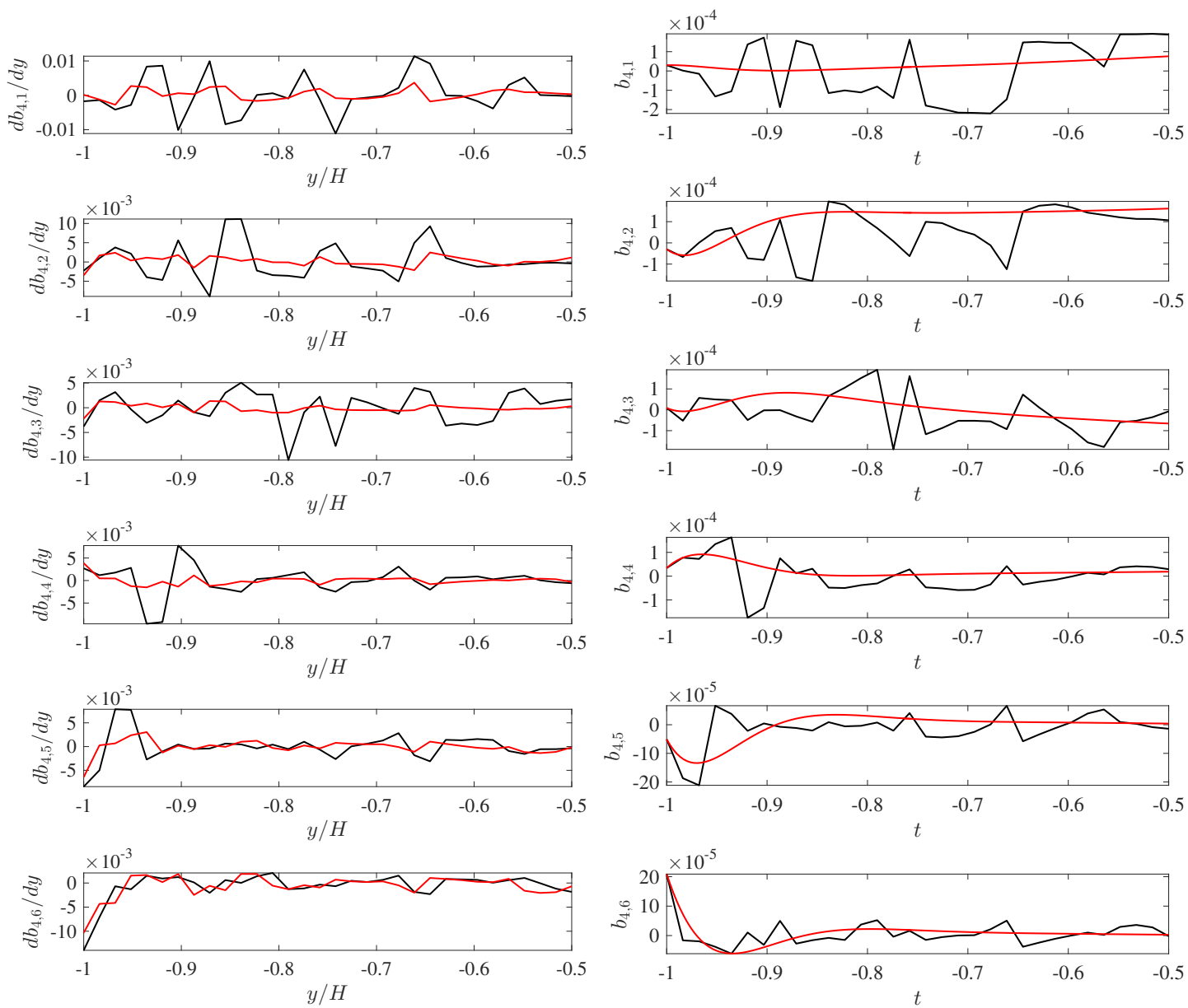

(A) $d \hat{b}_{4, m} / d y$

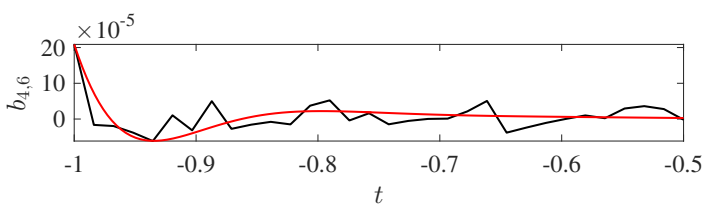

(B) $\hat{b}_{4, m}$

Figure C.1.12: LODS predictions for $\hat{b}_{4, m}$ (right), based on the parameter fit of $d \hat{b}_{4, m} / d y$ (left). 

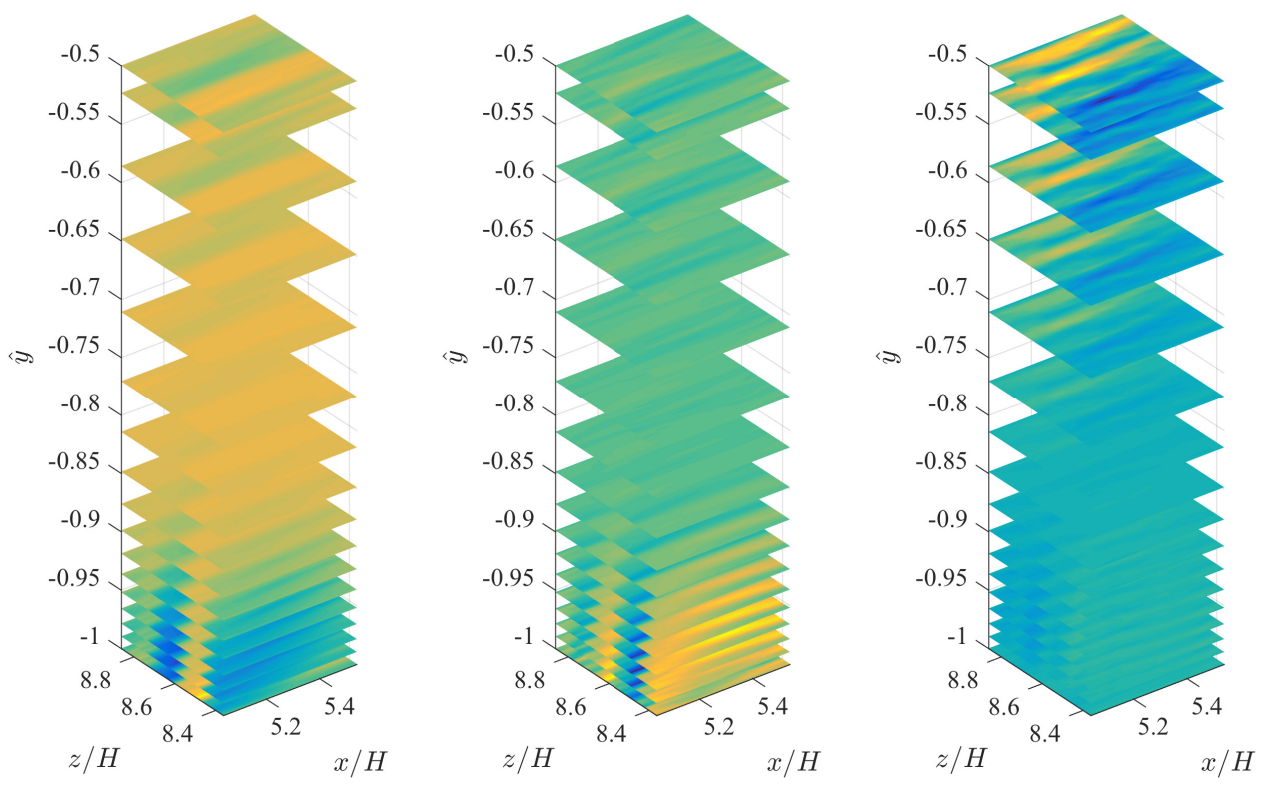

Figure C.2.13: DPOD LODS prediction of $\hat{\Phi}_{1}$ as a function of $\hat{y}$. From left are streamwise, wall-normal, and spanwise components.
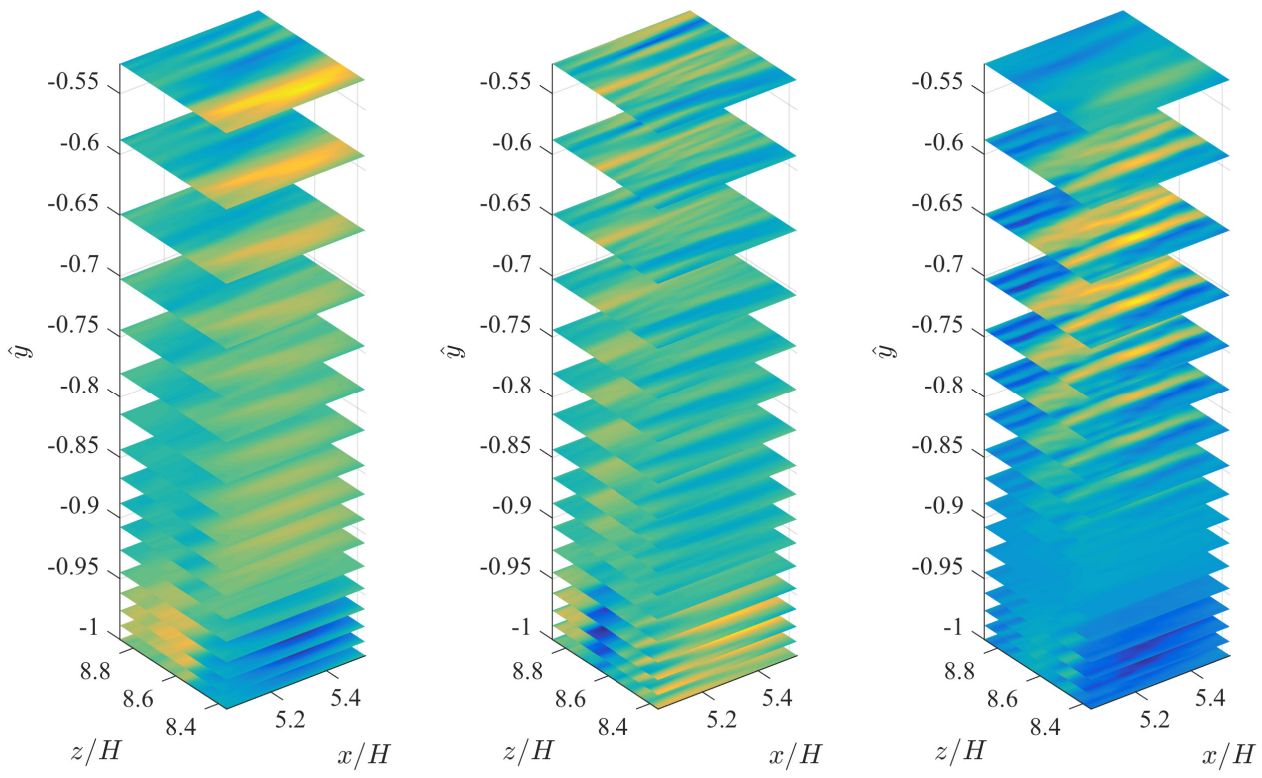

Figure C.2.14: DPOD LODS prediction of $\hat{\Phi}_{2}$ as a function of $\hat{y}$. From left are streamwise, wall-normal, and spanwise components. 

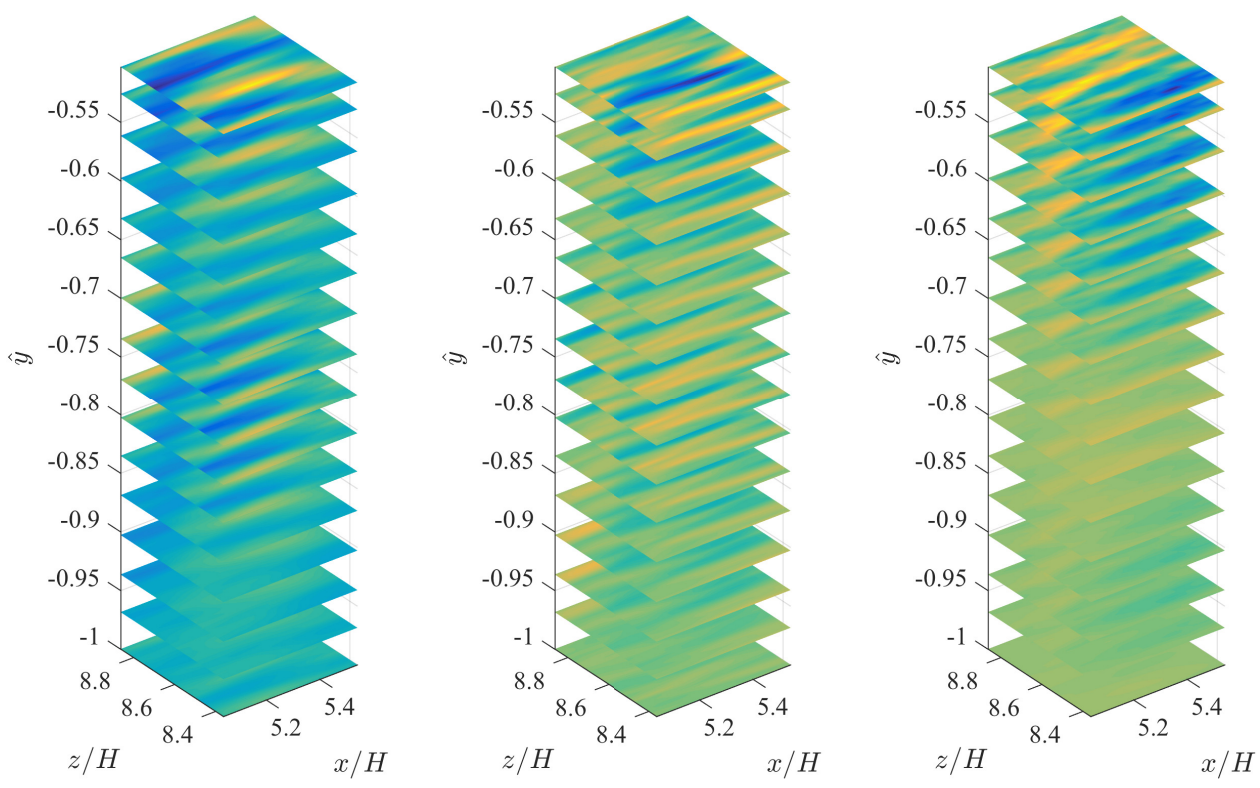

Figure C.2.15: DPOD LODS prediction of $\hat{\Phi}_{3}$ as a function of $\hat{y}$. From left are streamwise, wall-normal, and spanwise components.
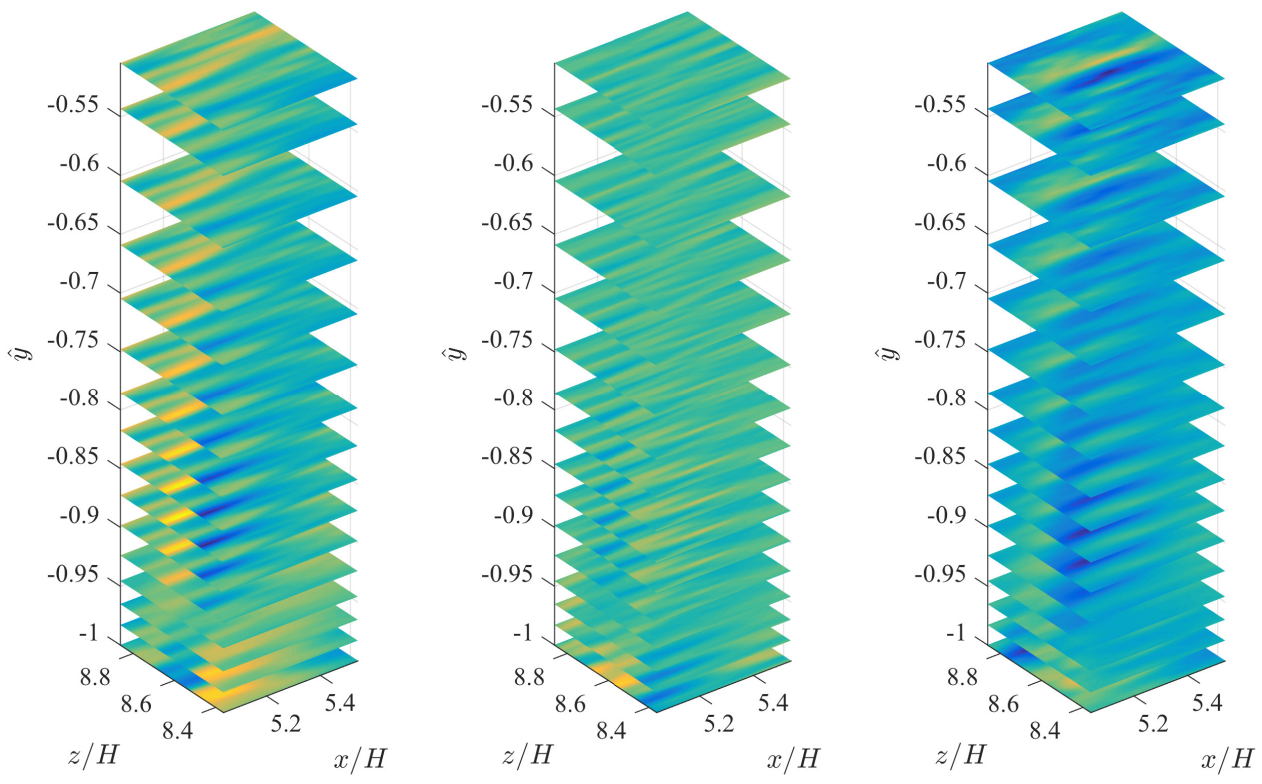

Figure C.2.16: DPOD LODS prediction of $\hat{\Phi}_{4}$ as a function of $\hat{y}$. From left are streamwise, wall-normal, and spanwise components. 

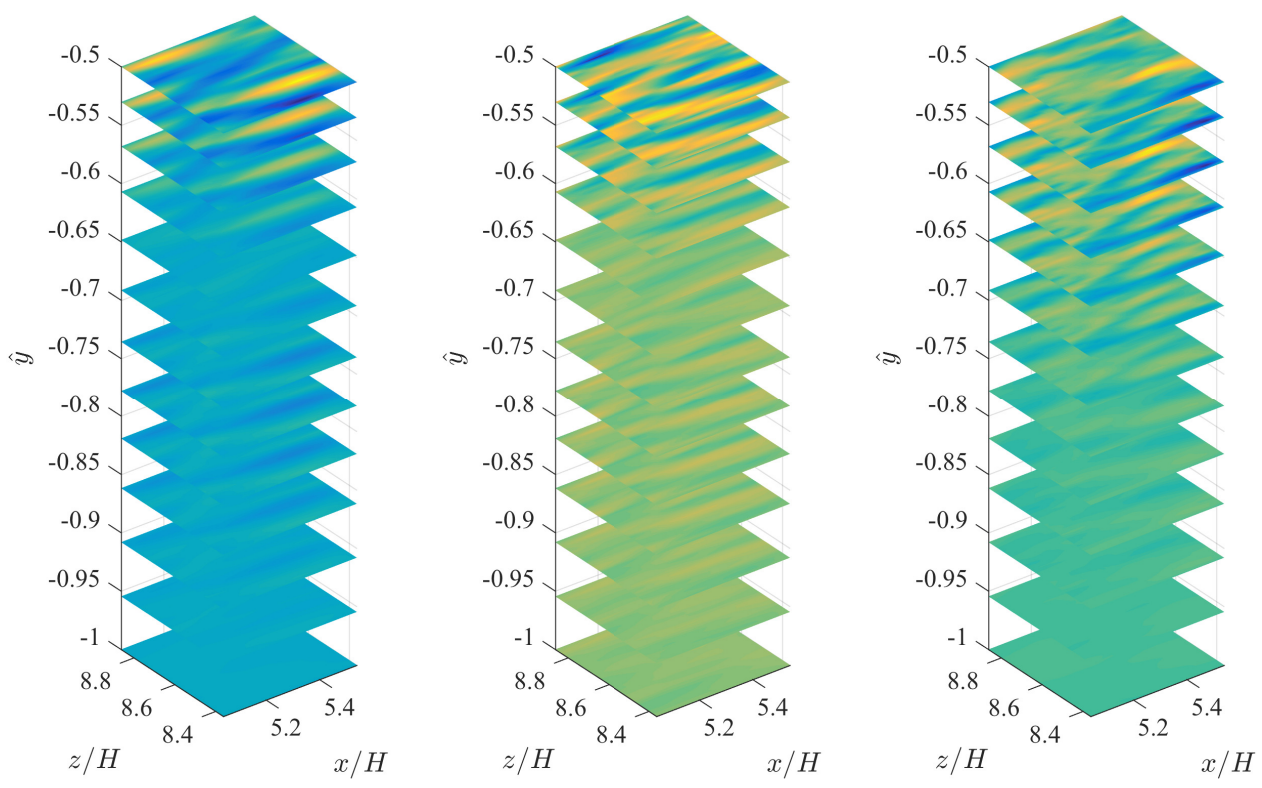

Figure C.2.17: DPOD LODS prediction of $\hat{\Phi}_{5}$ as a function of $\hat{y}$. From left are streamwise, wall-normal, and spanwise components.
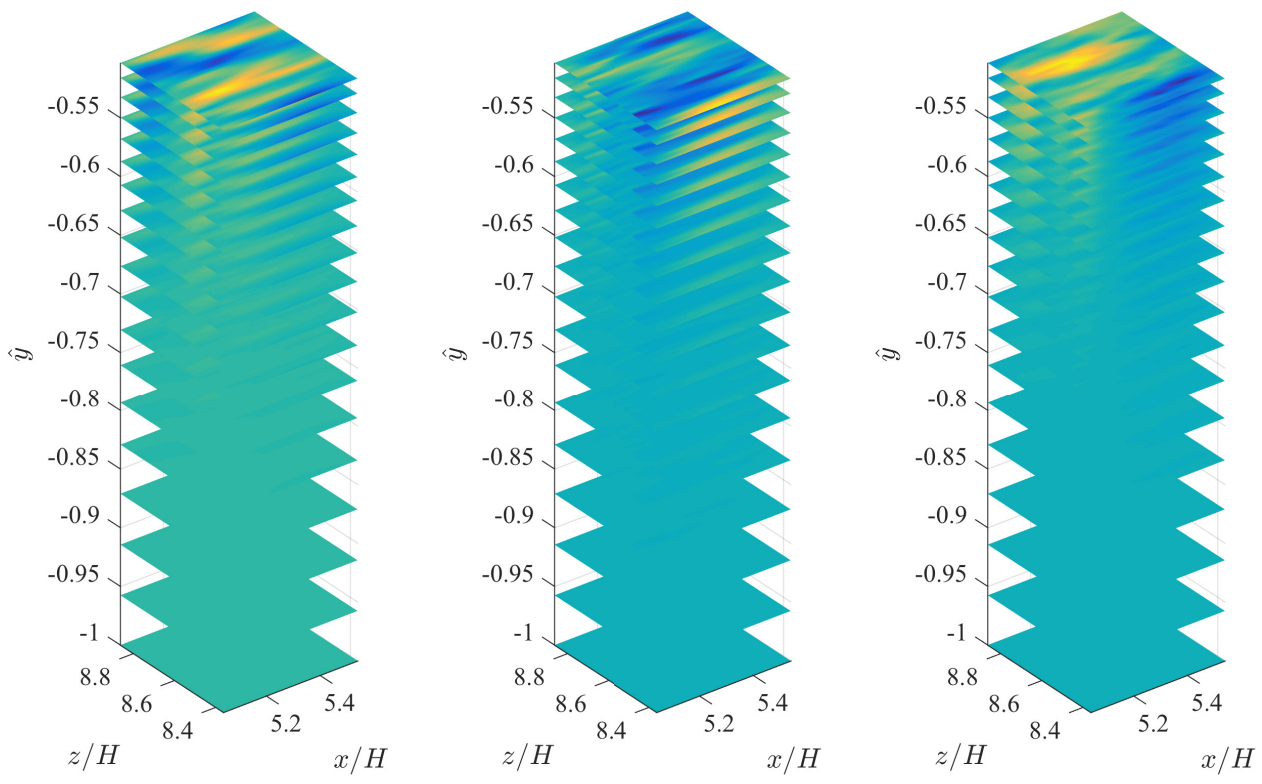

Figure C.2.18: DPOD LODS prediction of $\hat{\Phi}_{6}$ as a function of $\hat{y}$. From left are streamwise, wall-normal, and spanwise components. 


\section{Appendix D}

\section{Turbulent channel flow documentation}

The full description for the fully developed turbulent channel flow DNS is included below for reference. The included document is reproduced from literature at the Johns Hopkins turbulence database and may be sourced at http://turbulence.pha.jhu.edu/docs/README-CHANNEL.pdf. This document is referenced in the experiment section where the simulation is described \$3.2. Included are descriptions and figures of the flow more in accordance with traditional boundary layer and channel flow turbulence research. Because only small samples of the total data were taken for the current work, some of the data included in the documentation was out of reach, such as full profiles of the stress components and spectra. They are included here for completeness and to satisfy the curiosity of the reader. The author does not claim authorship of the documentation, and refers the reader to listed authors for any clarification. 


\title{
The JHU Turbulence Databases (JHTDB)
}

TURBULENT CHANNEL FLOW DATA SET

\author{
Data provenance: J. Graham ${ }^{1}$, M. Lee ${ }^{2}$, N. Malaya ${ }^{2}$, R.D. Moser $^{2}$, G. Eyink ${ }^{1} \&$ C. Meneveau ${ }^{1}$ \\ Database ingest and Web Services: K. Kanov ${ }^{1}$, R. Burns ${ }^{1}$, A. Szalay ${ }^{1}$ \& IDIES staff \\ ${ }^{1}$ Johns Hopkins University, Baltimore, MD 21218 \\ ${ }^{2}$ University of Texas, Austin, TX 78712
}

The turbulent channel flow database is produced from a direct numerical simulation (DNS) of wall bounded flow with periodic boundary conditions in the longitudinal and transverse directions, and no-slip conditions at the top and bottom walls. In the simulation, the Navier-Stokes equations are solved using a wall-normal, velocity-vorticity formulation [1]. Solutions to the governing equations are provided using a Fourier-Galerkin pseudo-spectral method for the longitudinal and transverse directions and seventh-order Basis-splines (B-splines) collocation method in the wall normal direction. Dealiasing is performed using the $3 / 2$-rule $[3]^{(1)}$. Temporal integration is performed using a low-storage, third-order Runge-Kutta method ${ }^{(2)}$. Initially, the flow is driven using a constant volume flux control (imposing a bulk channel mean velocity of $U=1$ ) until stationary conditions are reached. Then the control is changed to a constant applied mean pressure gradient forcing term equivalent to the shear stress resulting from the prior steps. Additional iterations are then performed to further achieve statistical stationarity before outputting fields.

The simulation is performed using the petascale DNS channel flow code (PoongBack) developed at the University of Texas at Austin by Prof. Robert Moser's research group [2]. In the wall-normal, velocity-vorticity formulation, the pressure is eliminated from the governing equations. In order to obtain the pressure field for the database, we subsequently implemented, in PoongBack, the pressure solver which solves the pressure Poisson equation given as

$$
\nabla^{2} p=-\nabla \cdot[\nabla \cdot(\boldsymbol{u} \otimes \boldsymbol{u})]
$$

where $p$ is the pressure divided by density, and $\boldsymbol{u}$ the velocity. The Neumann boundary condition, expressed as

$$
\frac{\partial p}{\partial y}=\nu \frac{\partial^{2} v}{\partial y^{2}}
$$

where $\nu$ is the molecular kinematic viscosity and $v$ the wall-normal velocity component, is used at the top and bottom walls. This calculation is performed independently from the velocity field solution only when outputting fields.

The simulation is performed for approximately a single flow through time. The 3 component velocity vector and pressure fields are stored every 5 time steps, resulting in 4000 frames of data. Information regarding the simulation setup and resulting statistical quantities are listed below.

Note that the averaging operation for mean and other statistical quantities is applied in time and over $x-z$ planes. 


\section{Simulation parameters}

- Domain Length: $L_{x} \times L_{y} \times L_{z}=8 \pi h \times 2 h \times 3 \pi h$ where $h$ is the half-channel height $(h=1$ in dimensionless units)

- Grid: $N_{x} \times N_{y} \times N_{z}=2048 \times 512 \times 1536$ (wavemodes); $3072 \times 512 \times 2304$ (collocation points); data is stored at the wavemode resolution, i.e. $N_{x} \times N_{y} \times N_{z}=2048 \times 512 \times 1536$ at grid point nodes in physical space.

- Viscosity: $\nu=5 \times 10^{-5}$ (non-dimensional)

- Mean pressure gradient: $d P / d x=0.0025$ (non-dimensional)

- DNS Time step: $\Delta t=0.0013$ (non-dimensional)

- Database time step: $\delta t=0.0065$ (non-dimensional)

- Time stored: $t=[0,25.9935]$

Flow statistics averaged over $t=[0,26]$

- Bulk velocity: $U_{b}=0.99994$

- Centerline velocity: $U_{c}=1.1312$

- Friction velocity: $u_{\tau}=4.9968 \times 10^{-2}$

- Viscous length scale: $\delta_{\nu}=\nu / u_{\tau}=1.0006 \times 10^{-3}$

- Reynolds number based on bulk velocity and full channel height: $R e_{b}=\frac{U_{b} 2 h}{\nu}=3.9998 \times 10^{4}$

- Centerline Reynolds number: $R e_{c}=U_{c} h / \nu=2.2625 \times 10^{4}$

- Friction velocity Reynolds number: $R e_{\tau}=u_{\tau} h / \nu=9.9935 \times 10^{2}$

\section{Grid spacing in viscous units}

- $x$ direction: $\Delta x^{+}=12.2639$

- $y$ direction at first point: $\Delta y_{1}^{+}=1.65199 \times 10^{-2}$

- $y$ direction at center: $\Delta y_{c}^{+}=6.15507$

- $z$ direction: $\Delta z^{+}=6.13196$

In the following figures several quantities from the simulation are show. Shown in Figure 1 is the computed friction Reynolds for the time interval in the database. In Figure 2 the mean velocity is show along with the standard $U^{+}$profiles in the viscous sublayer and log-layer. The viscous and turblent shear stresses, Reynolds normal stresses, mean pressure, pressure variance, and velocity-pressure covariances are shown in Figures 3-6. In the remaining plots, the power spectral densities of velocity and pressure are shown for various $y^{+}$locations. Streamwise spectra are shown in Figure 7, whereas span wise spectra are shown in Figure 8.

Acknowledgements: The JHU team acknowledges funding by the National Science Foundation, CMMI-0941530. The University of Texas team acknowledges funding from the National Science Foundation PetaApps Grant OCI-0749223 and PRAC Grant 0832634, which supported the development of the PoongBack code. 
(1) Note: The divergence-free condition in the simulation is enforced based on the spectral representation of the derivatives. The JHTDB analysis tools for gradients are based on finite differencing of various orders. Therefore, when evaluating the divergence using these spatially more localized derivative operators, a non-negligible error in the divergence is obtained, as expected.

(2) Note (for patch added June 1, 2014): The simulation was performed in a frame moving in the $x$-direction at a speed $U_{\text {frame }}=0.45$. The velocity values stored in the database are the velocities as seen in a frame attached to the stationary channel walls, i.e. we stored $(u, v, w)=\left(u_{\text {DNS }}+\right.$ $\left.0.45, v_{\mathrm{DNS}}, w_{\mathrm{DNS}}\right)$, where $\left(u_{\mathrm{DNS}}, v_{\mathrm{DNS}}, w_{\mathrm{DNS}}\right)$ are the velocity components computed in the DNS, and $(u, v, w)$ are the velocity components stored in the database. The spatial locations where the data are ingested into the database are the values at the (moving) grid locations ( $\left.x_{\mathrm{DNS}}, y_{\mathrm{DNS}}, z_{\mathrm{DNS}}\right)$. We apply the Galilean transformation $(x, y, z ; t)=\left(x_{\mathrm{DNS}}+0.45 t, y_{\mathrm{DNS}}, z_{\mathrm{DNS}} ; t_{\mathrm{DNS}}\right)$, where $(x, y, z ; t)$ is the position and time in the frame in which the wall is stationary, while $\left(x_{\mathrm{DNS}}, y_{\mathrm{DNS}}, z_{\mathrm{DNS}} ; t_{\mathrm{DNS}}\right)$ are the DNS (computational grid) coordinates and time. Users making a query for position $x$ at time $t$ will automatically receive the DNS value stored at $x_{\text {DNS }}=x-0.45 t$ for time $t$. For queries in which no spatial interpolation is specified, the query returns the nearest value on the DNS grid. For queries with spatial interpolation options, the requested interpolation is used based on the stored DNS values. (Before the corrective patch applied on June 1, 2014, the database returned the data at $x_{\mathrm{DNS}}=x$ instead of $\left.x_{\mathrm{DNS}}=x-0.45 t\right)$.

For the cutout service, the data are returned as stored on the nodes of the moving grid. That is to say, requests to grid a grid-point with index $i_{x}$ (where $i_{x}$ can take on values $(0,1,2,3, \ldots 2047)$ ) and at a time index $m_{t}$ (where $m_{t}$ can be $(0,1,2,3 . .1996)$ ) will correspond to a streamwise spatial location $x=i_{x} \Delta x+0.45\left(m_{t} \delta t\right)$. The other directions are unchanged, i.e. the $y$ location is $y=j_{y} \Delta y$ and $z=k_{z} \Delta z$; and time is given by $t=m_{t} \delta t$.

\section{References}

[1] J. Kim, P. Moin, and R. Moser. Turbulence statistics in fully developed channel flow at low Reynolds number. J. Fluid Mech., 177:133-166, 1987.

[2] M. Lee, N. Malaya, and R. D. Moser. Petascale direct numerical simulation of turbulent channel flow on up to 786k cores. Supercomputing (SC13), 2013.

[3] S. A. Orszag. On the elimination of aliasing in finite-difference schemes by filtering highwavenumber components. J. Atmos. Sci., 28:1074-1074, 1971. 


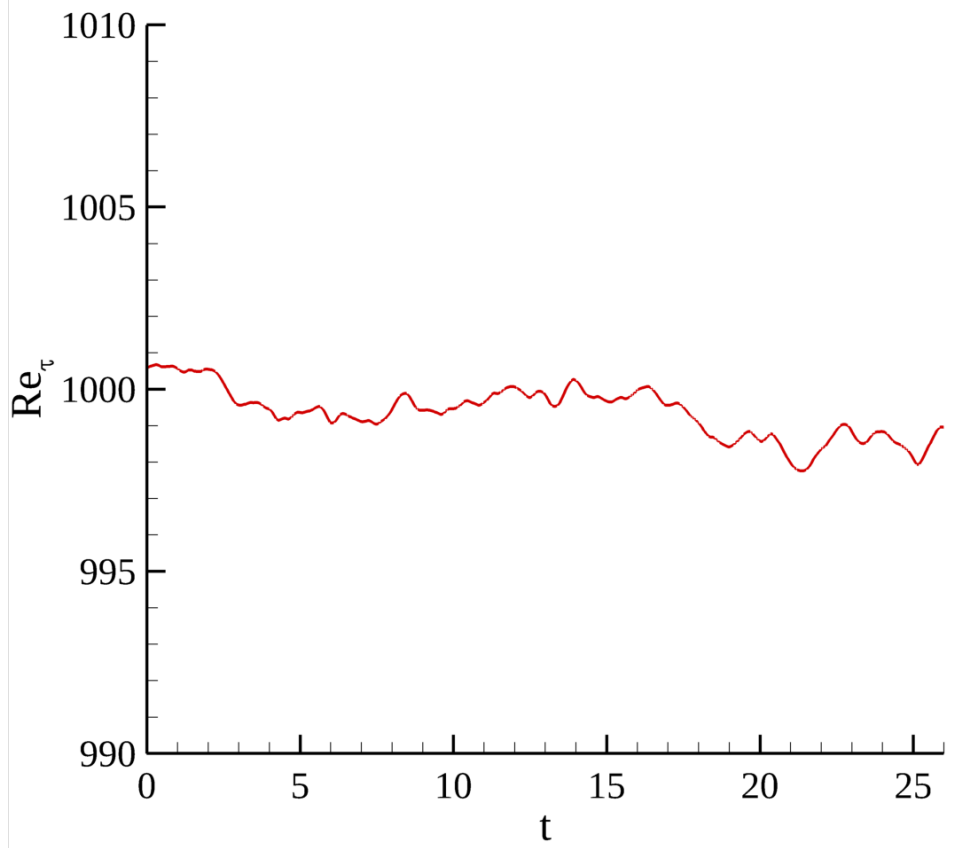

Figure 1: Friction velocity Reynolds number during the channel flow simulation during the database time interval 


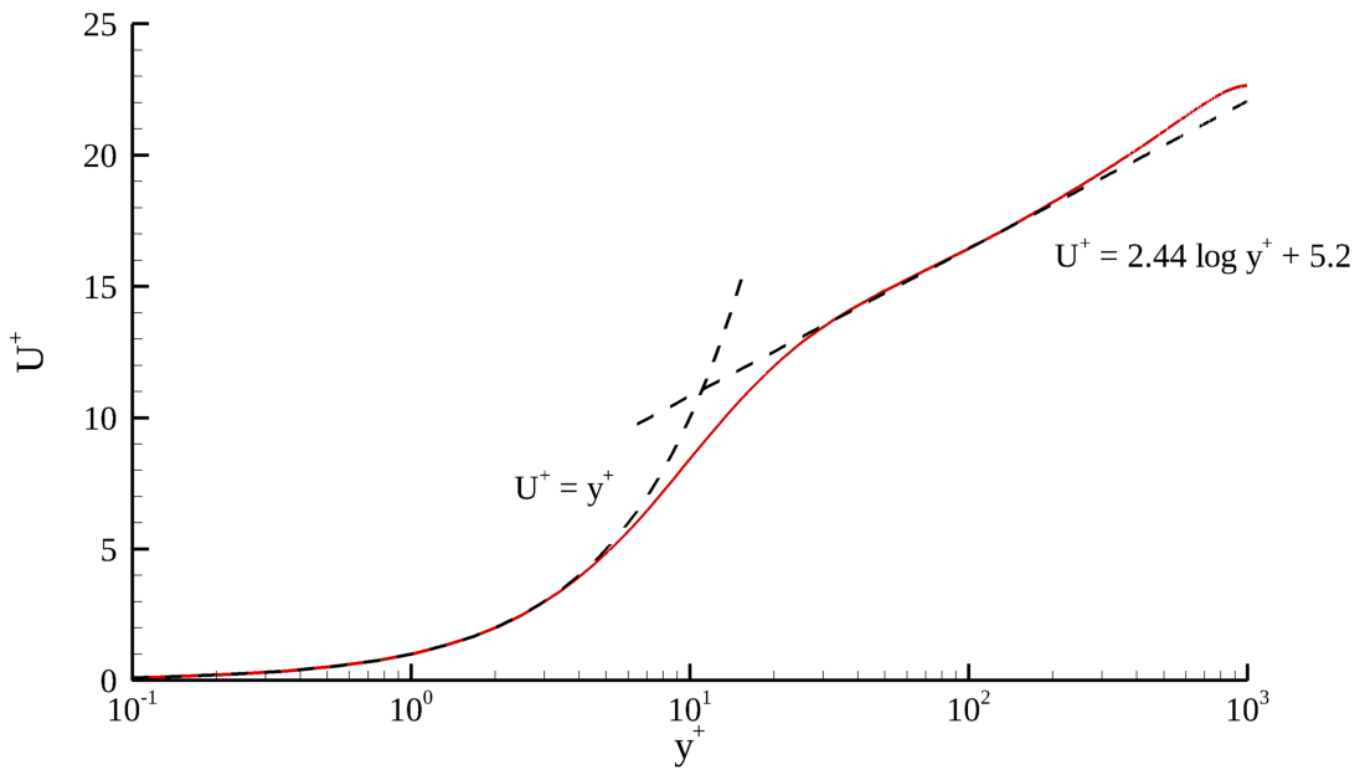

Figure 2: Mean velocity profile in viscous units. Standard values of $\kappa=0.41$ and $B=5.2$ are used in the log-law (dashed line) for reference. 


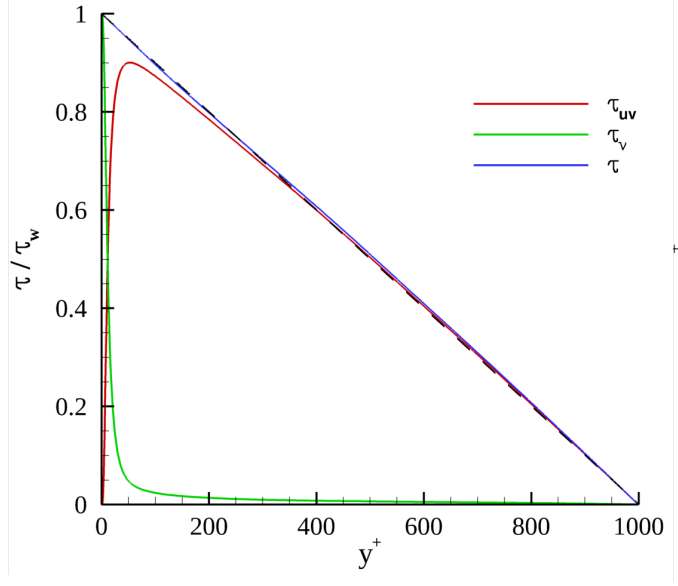

Figure 3: Mean viscous, turbulent, and total shear stress normalized by the wall stress

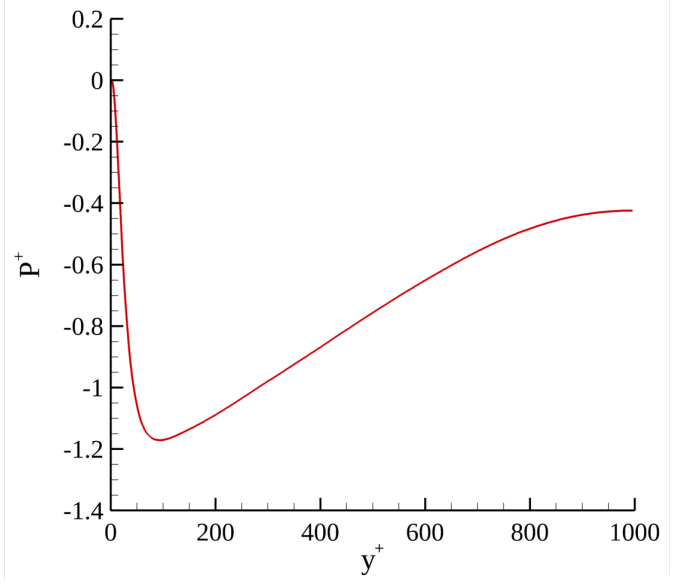

Figure 5: Mean pressure profile in viscous units

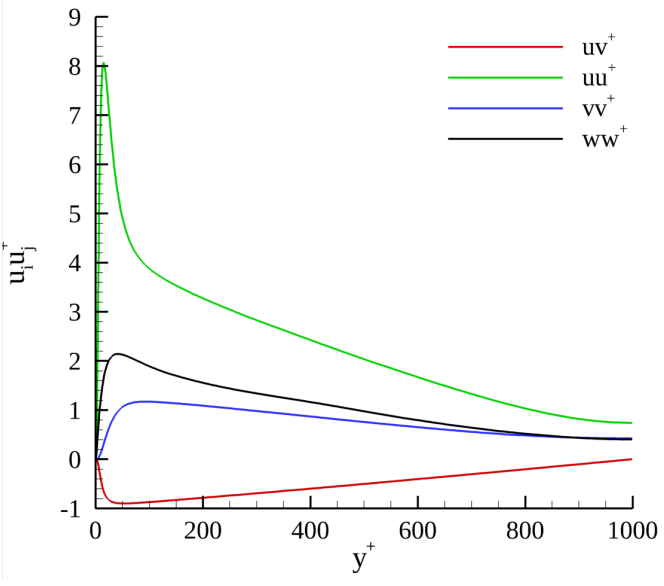

Figure 4: Velocity covariances in viscous units

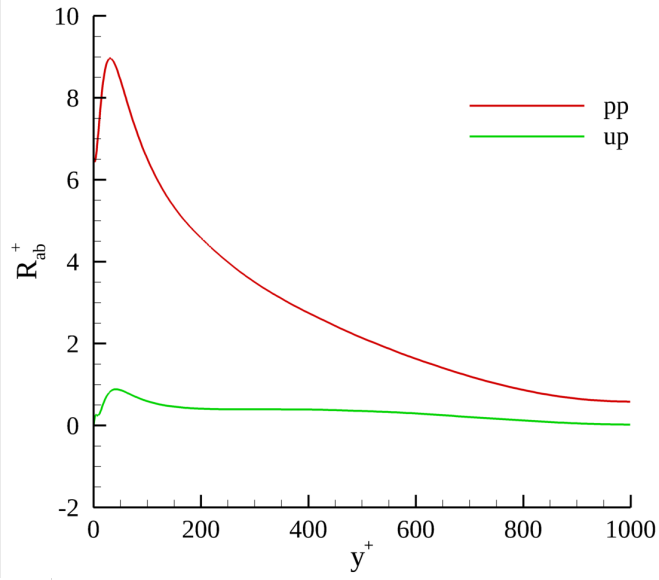

Figure 6: Pressure variance and pressurevelocity covariance in viscous units 


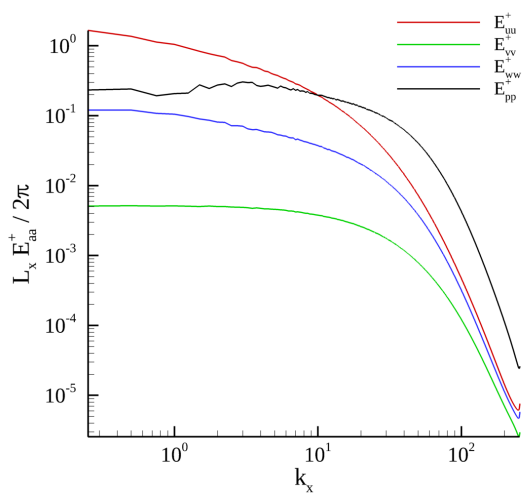

(a) $y^{+}=10.11$

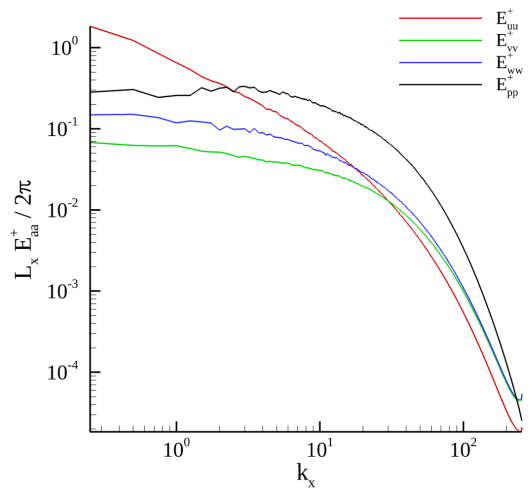

(c) $y^{+}=99.75$

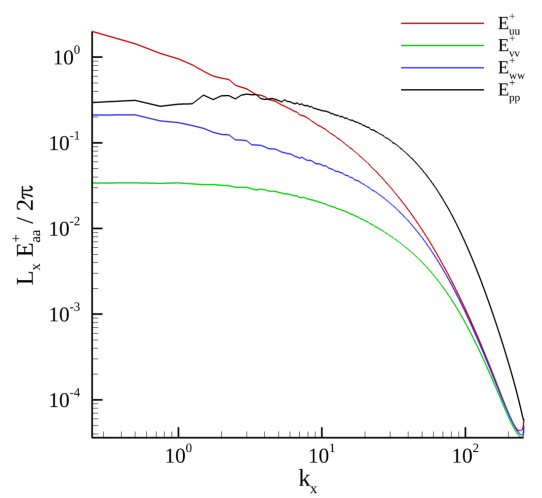

(b) $y^{+}=29.89$

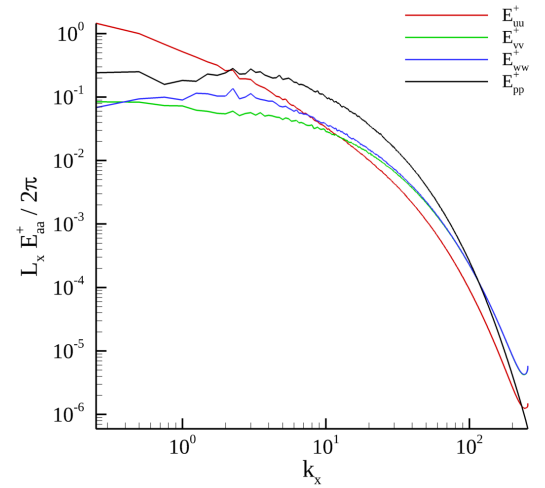

(d) $y^{+}=371.6$

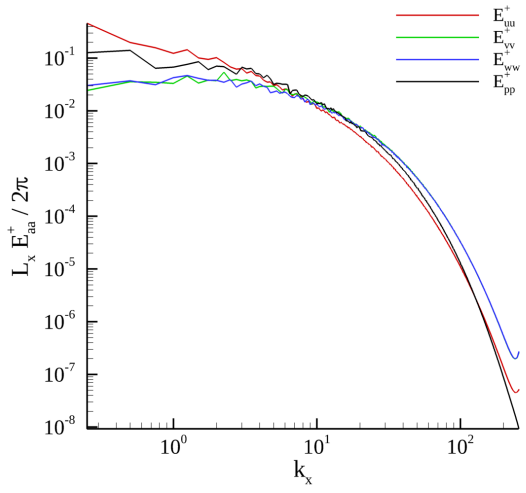

(e) $y^{+}=999.7$

Figure 7: Streamwise power spectral densities at various $y^{+}$locations as function of $k_{x}$ 


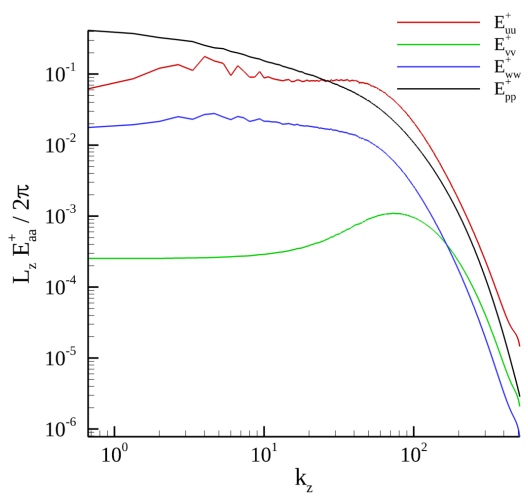

(a) $y^{+}=10.11$

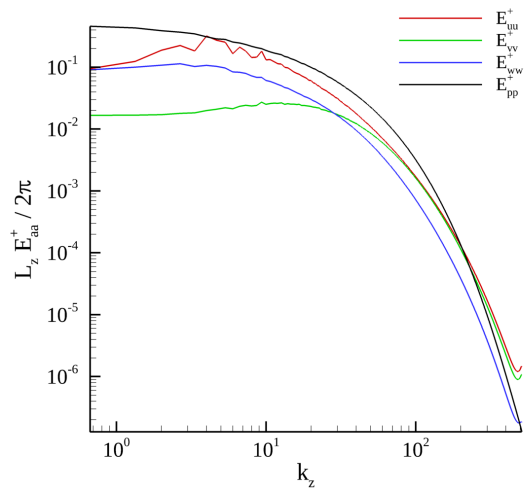

(c) $y^{+}=99.75$

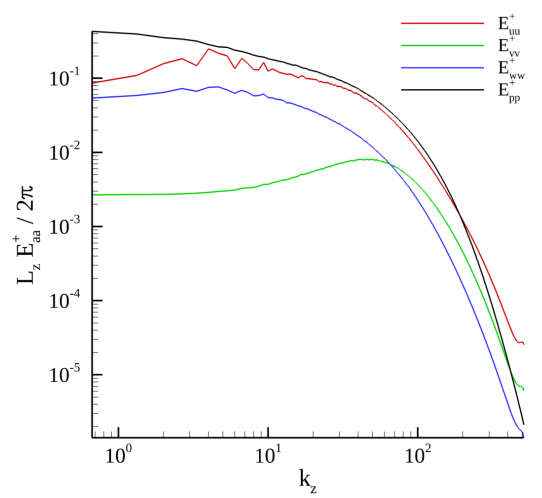

(b) $y^{+}=29.89$

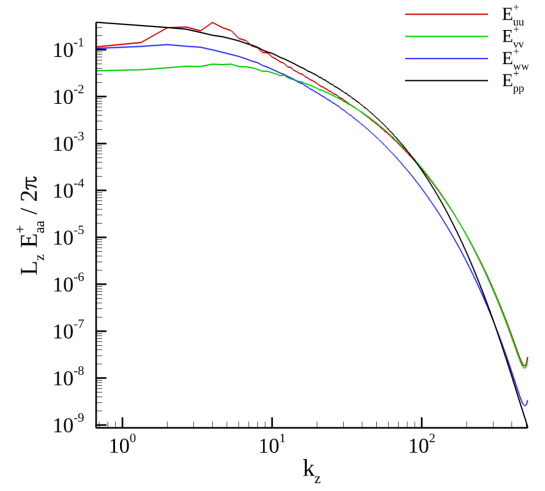

(d) $y^{+}=371.6$

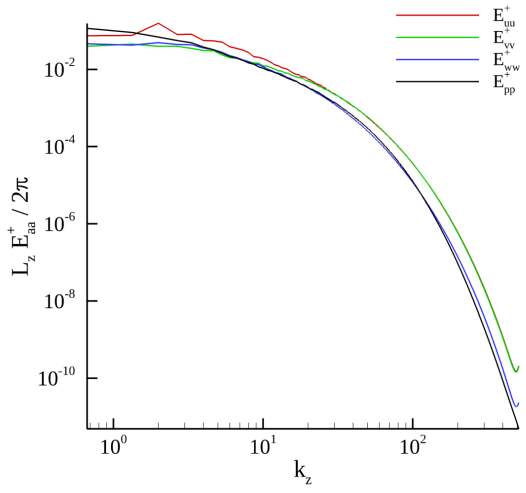

(e) $y^{+}=999.7$

Figure 8: Spanwise power spectral densities at various $y^{+}$locations as function of $k_{z}$ 


\section{Appendix E}

\section{Open-loop transfer functions}

In the system used to predict initial condition for the LODS of the wind turbine wake, open loop transfer functions were employed to correlate the dissimilar input and output signals. In each case, the System Identification Toolbox supplied with Matlab was used to identify Poles and zeros for each transfer function. For each mode coefficient $(i \in[1 \ldots 14])$, transfer functions were estimated for a range of poles and zeros. The number of poles was arbitrarily limited to a maximum of $n_{p}=10$, and the number of zeros was limited to $n_{z}=n_{p}-1=9$. Goodness of fit was calculated between predicted outcomes of each transfer function and validation data taken as the POD coefficients. The following figures shown the pole-zero maps of selected transfer functions as well as the Bode plots showing magnitude and phase of the frequency response of each transfer function. Optimized transfer functions exhibit as many as 10 poles and 9 zero locations, and the fit ranges between 40 and $80 \%$. Table E.1 shows details of the transfer functions employed in the recalibration process.

Table E.1: Number of poles, zeros, and the goodness of fit for transfer functions relating $U_{\text {eff }}$ to each mode coefficient.

\begin{tabular}{c|rrrrrrr} 
Mode & 1 & 2 & 3 & 4 & 5 & 6 & 7 \\
Poles & 7 & 10 & 10 & 8 & 10 & 10 & 10 \\
Zeros & 2 & 5 & 4 & 7 & 8 & 9 & 8 \\
Fit (\%) & 78.37 & 81.43 & 65.18 & 64.24 & 63.48 & 71.53 & 56.70 \\
& & & & & & & \\
Mode & 8 & 9 & 10 & 11 & 12 & 13 & 14 \\
Poles & 10 & 9 & 9 & 10 & 10 & 9 & 10 \\
Zeros & 6 & 7 & 8 & 7 & 7 & 8 & 9 \\
Fit (\%) & 60.16 & 50.42 & 54.90 & 50.37 & 54.83 & 50.67 & 41.42
\end{tabular}



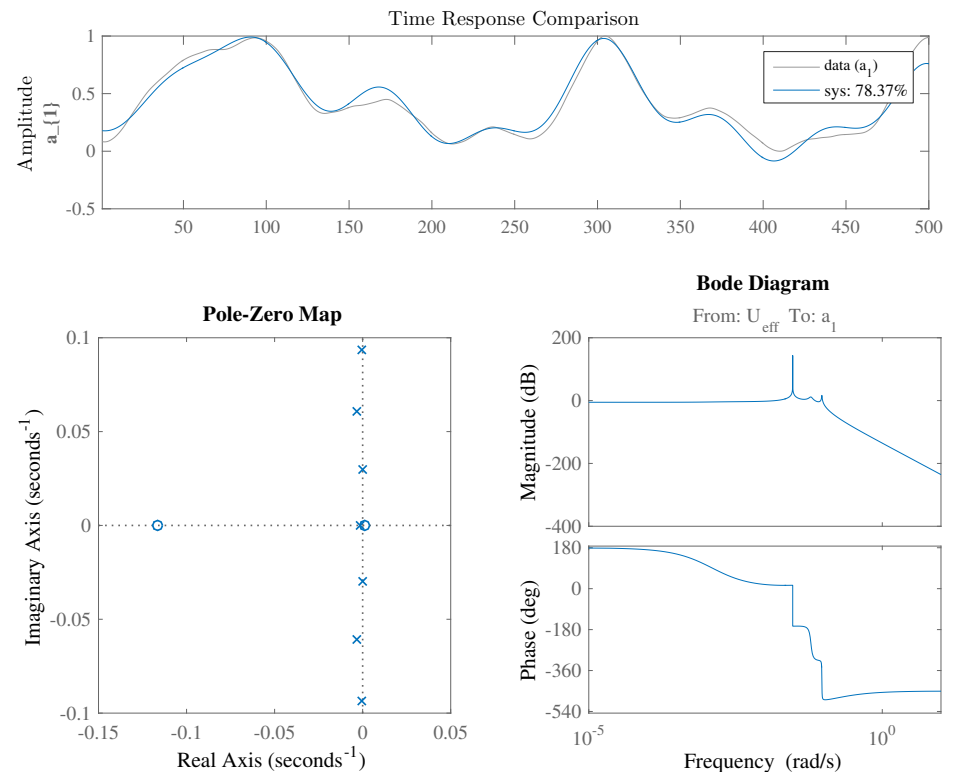

Figure E.0.1: Time response, pole-zero map, and Bode plot of the open-loop transfer function relating the effective inflow velocity $U_{\text {eff }}$ to the mode coefficient $a_{1}$.
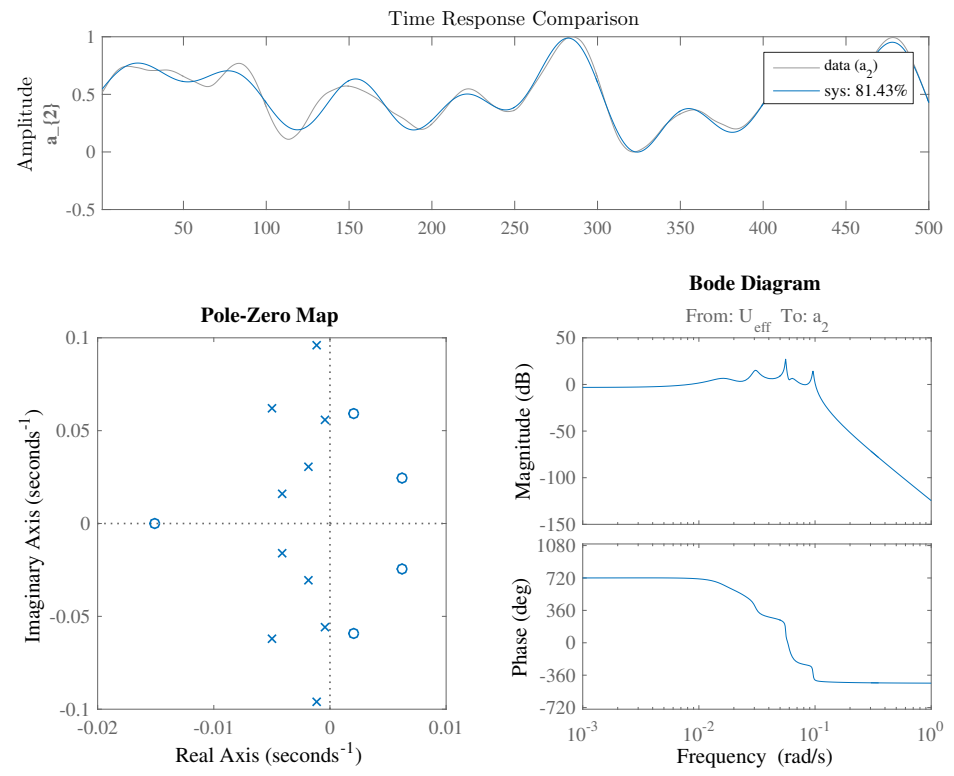

Figure E.0.2: Time response, pole-zero map, and Bode plot of the open-loop transfer function relating the effective inflow velocity $U_{\text {eff }}$ to the mode coefficient $a_{2}$. 

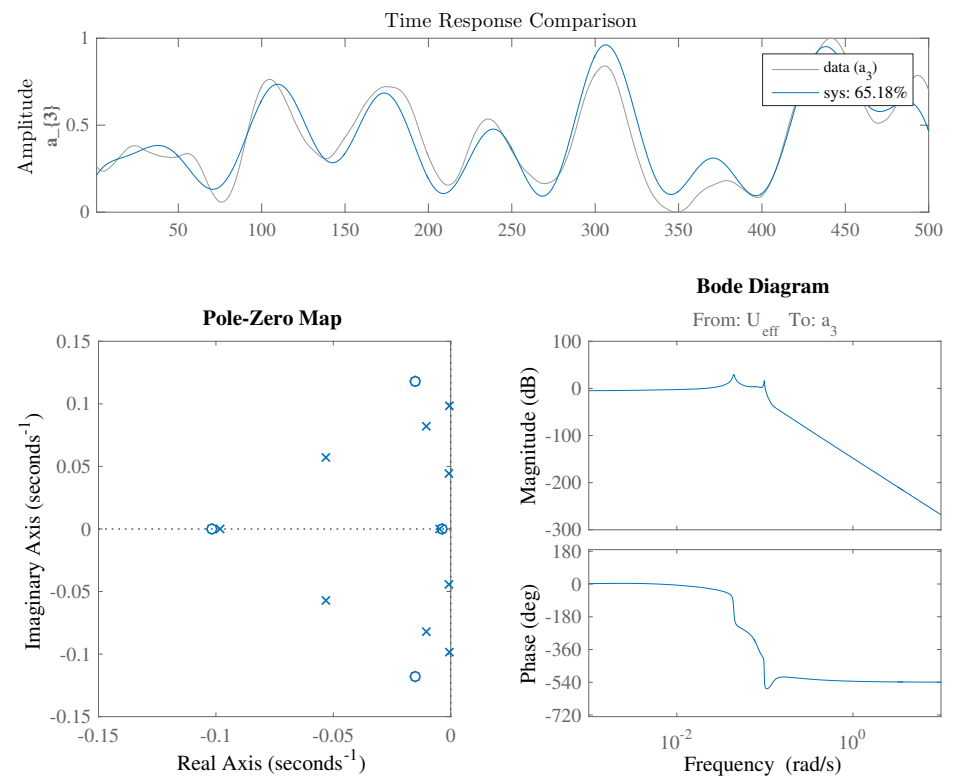

Figure E.0.3: Time response, pole-zero map, and Bode plot of the open-loop transfer function relating the effective inflow velocity $U_{\text {eff }}$ to the mode coefficient $a_{3}$.
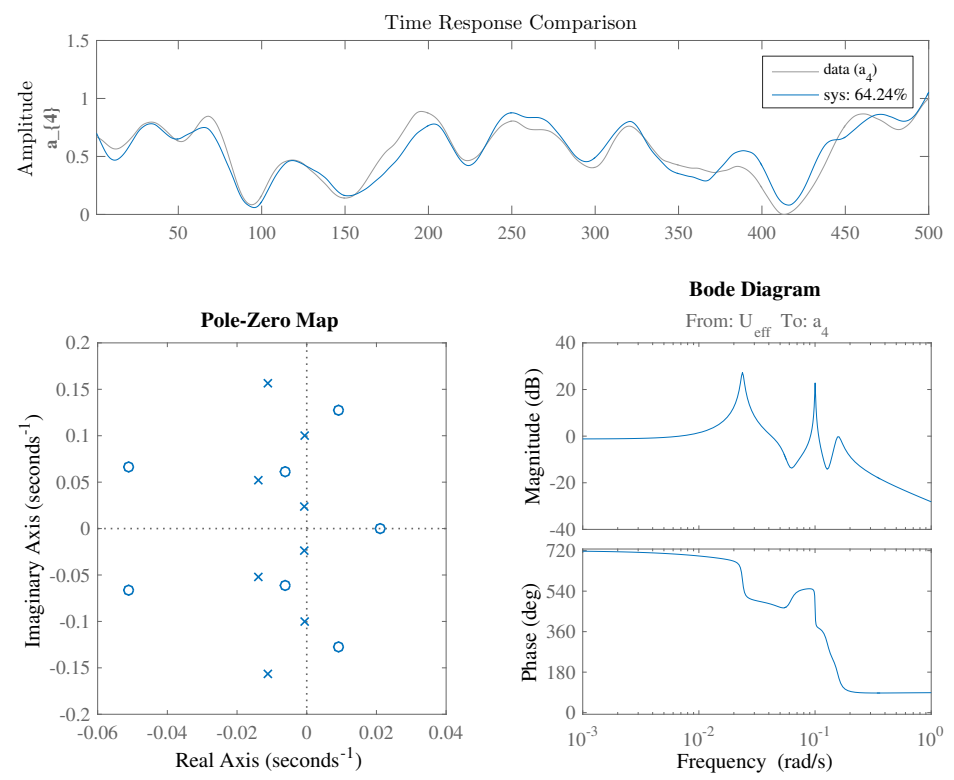

Figure E.0.4: Time response, pole-zero map, and Bode plot of the open-loop transfer function relating the effective inflow velocity $U_{\text {eff }}$ to the mode coefficient $a_{4}$. 

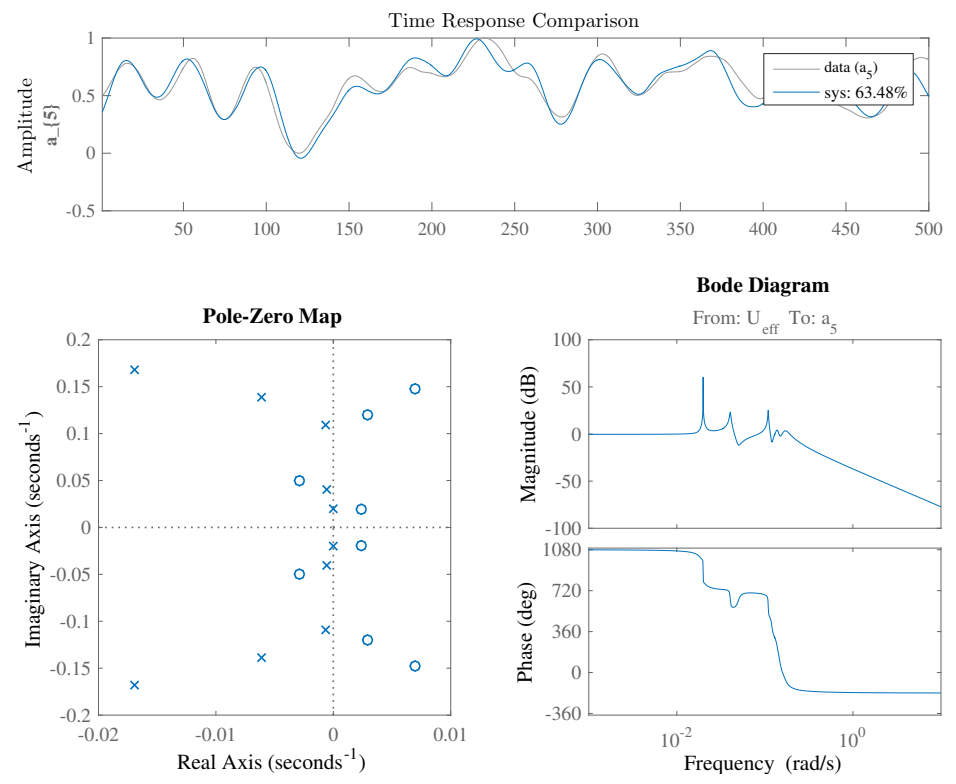

Figure E.0.5: Time response, pole-zero map, and Bode plot of the open-loop transfer function relating the effective inflow velocity $U_{\text {eff }}$ to the mode coefficient $a_{5}$.
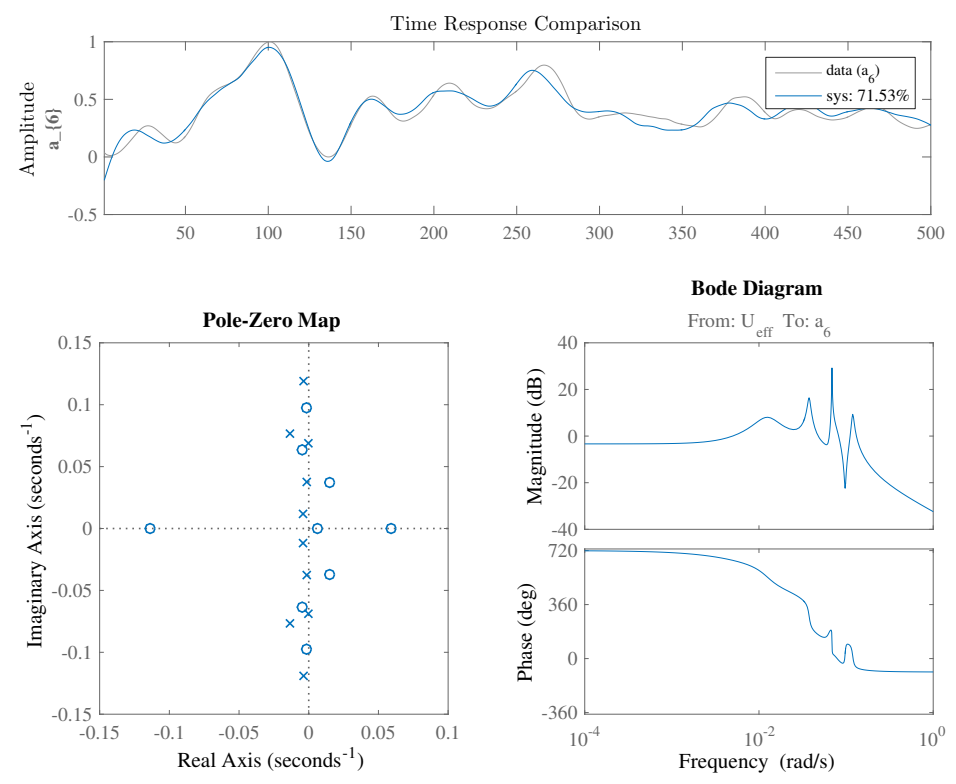

Figure E.0.6: Time response, pole-zero map, and Bode plot of the open-loop transfer function relating the effective inflow velocity $U_{\text {eff }}$ to the mode coefficient $a_{6}$. 

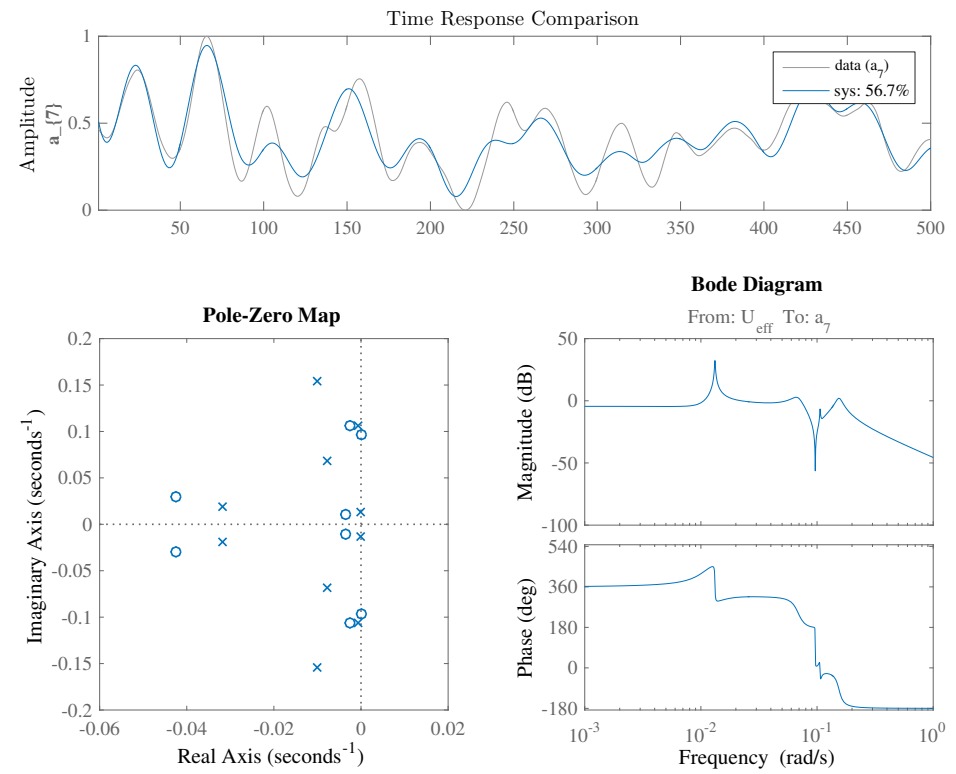

Figure E.0.7: Time response, pole-zero map, and Bode plot of the open-loop transfer function relating the effective inflow velocity $U_{\text {eff }}$ to the mode coefficient $a_{7}$.
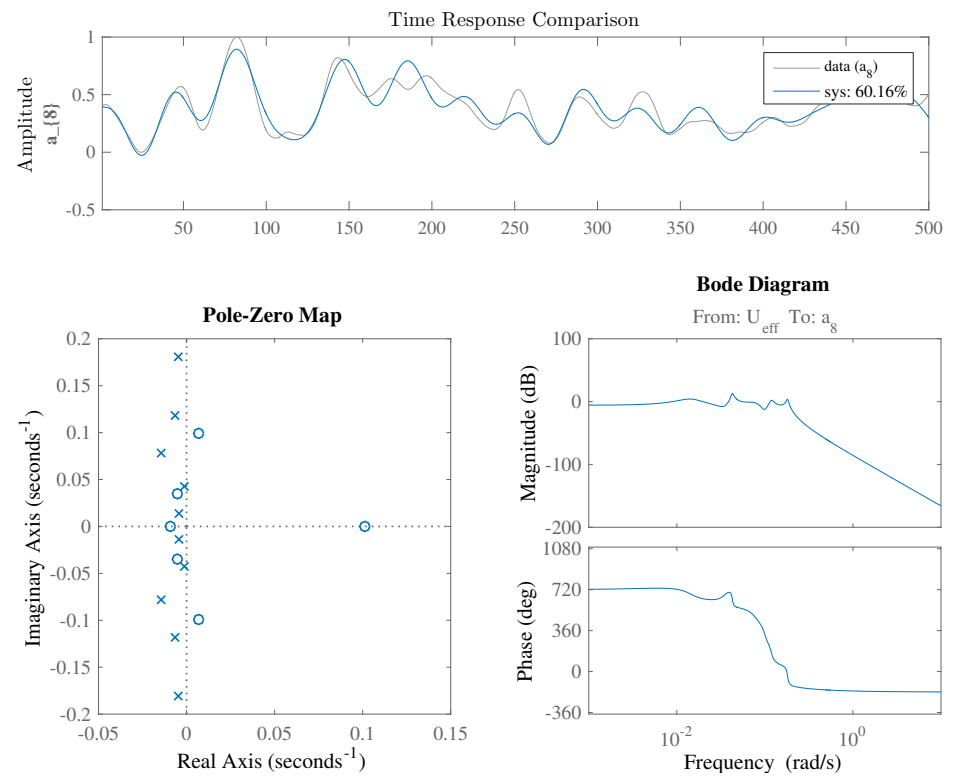

Figure E.0.8: Time response, pole-zero map, and Bode plot of the open-loop transfer function relating the effective inflow velocity $U_{\text {eff }}$ to the mode coefficient $a_{8}$. 

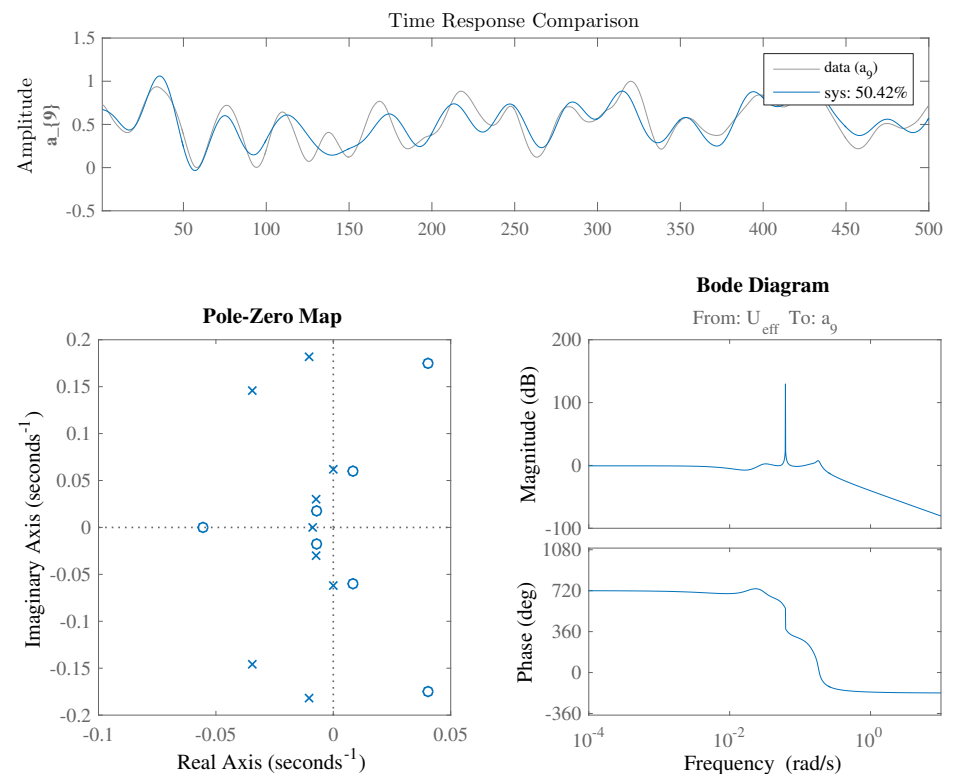

Figure E.0.9: Time response, pole-zero map, and Bode plot of the open-loop transfer function relating the effective inflow velocity $U_{\text {eff }}$ to the mode coefficient $a_{9}$.
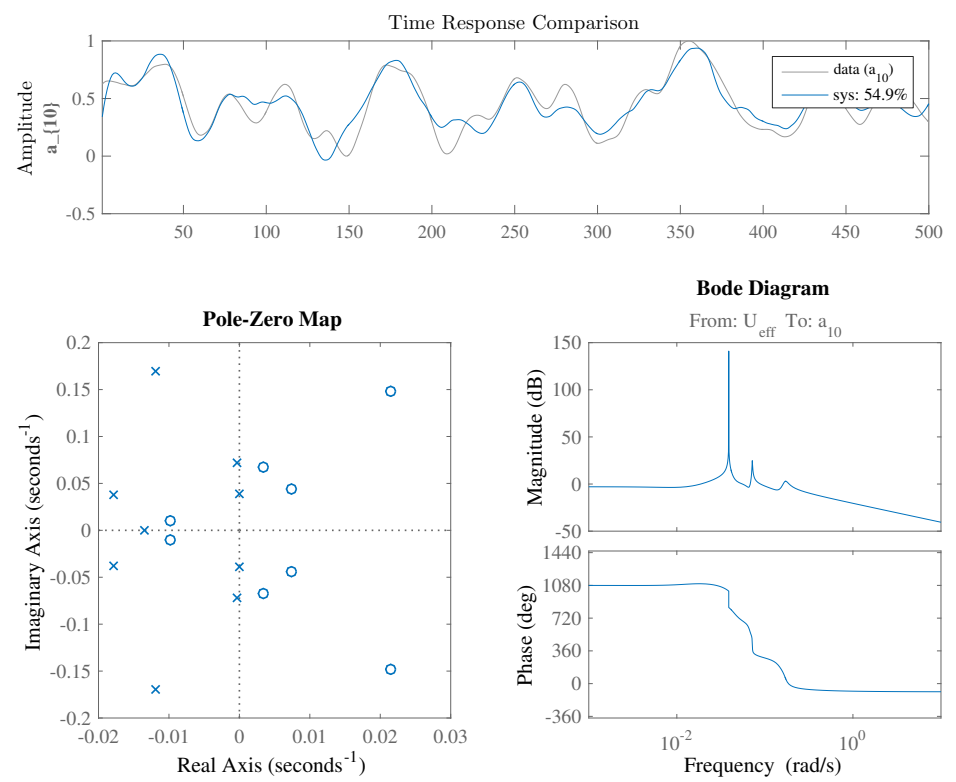

Figure E.0.10: Time response, pole-zero map, and Bode plot of the open-loop transfer function relating the effective inflow velocity $U_{\text {eff }}$ to the mode coefficient $a_{10}$. 

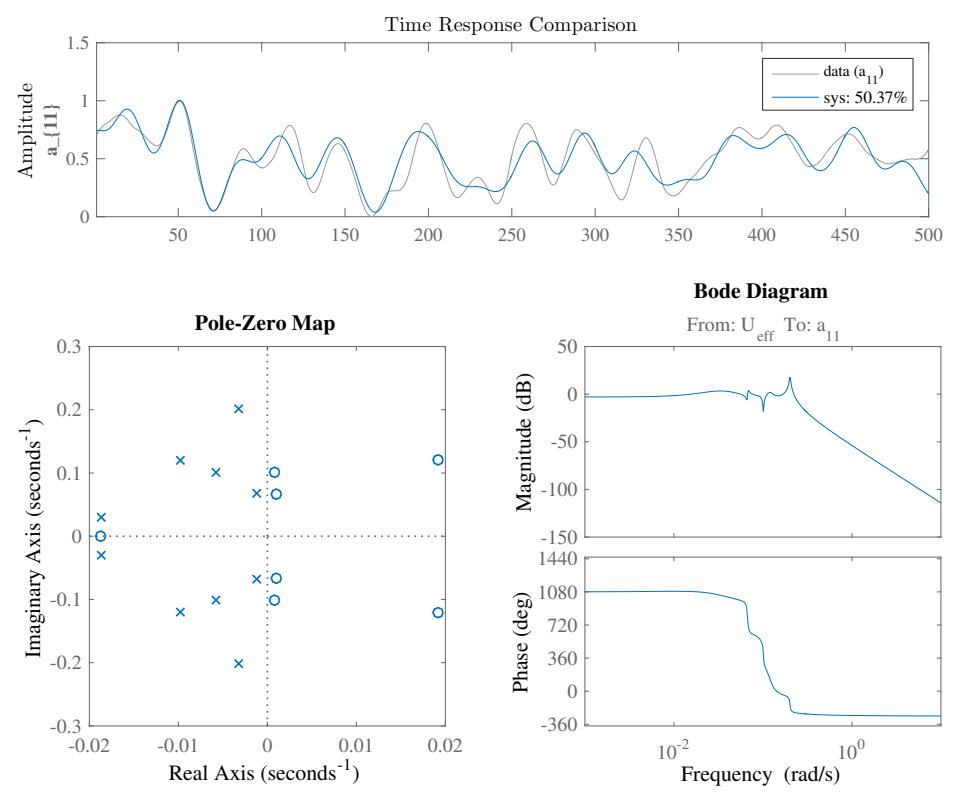

Figure E.0.11: Time response, pole-zero map, and Bode plot of the open-loop transfer function relating the effective inflow velocity $U_{\text {eff }}$ to the mode coefficient $a_{11}$.
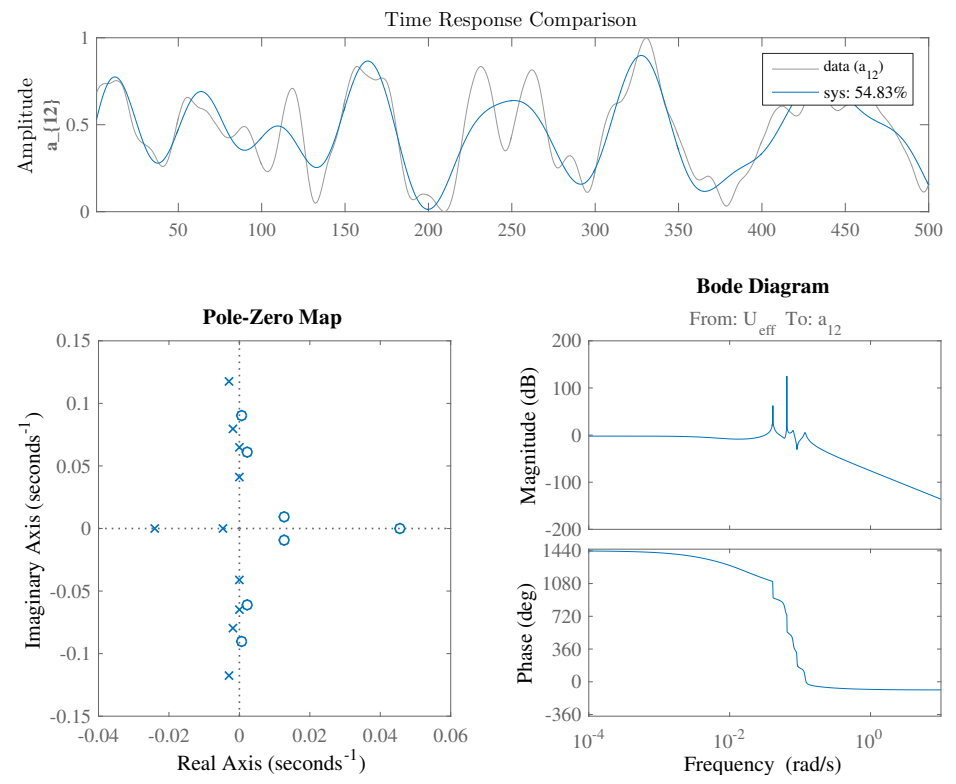

Figure E.0.12: Time response, pole-zero map, and Bode plot of the open-loop transfer function relating the effective inflow velocity $U_{\text {eff }}$ to the mode coefficient $a_{12}$. 

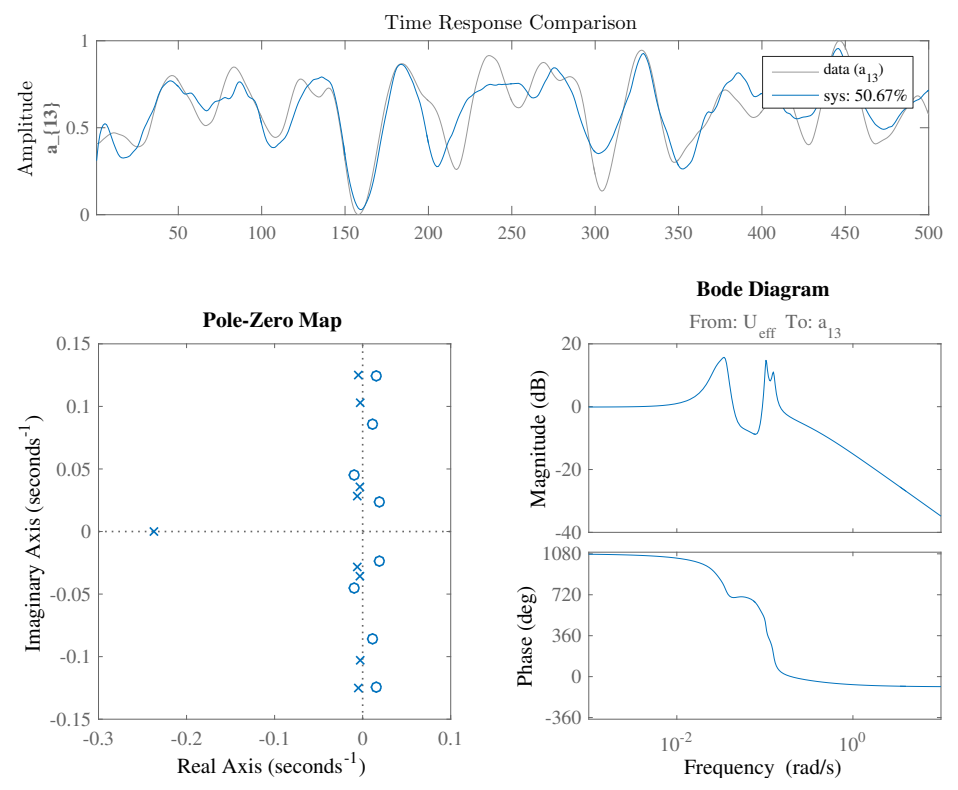

Figure E.0.13: Time response, pole-zero map, and Bode plot of the open-loop transfer function relating the effective inflow velocity $U_{\text {eff }}$ to the mode coefficient $a_{13}$.
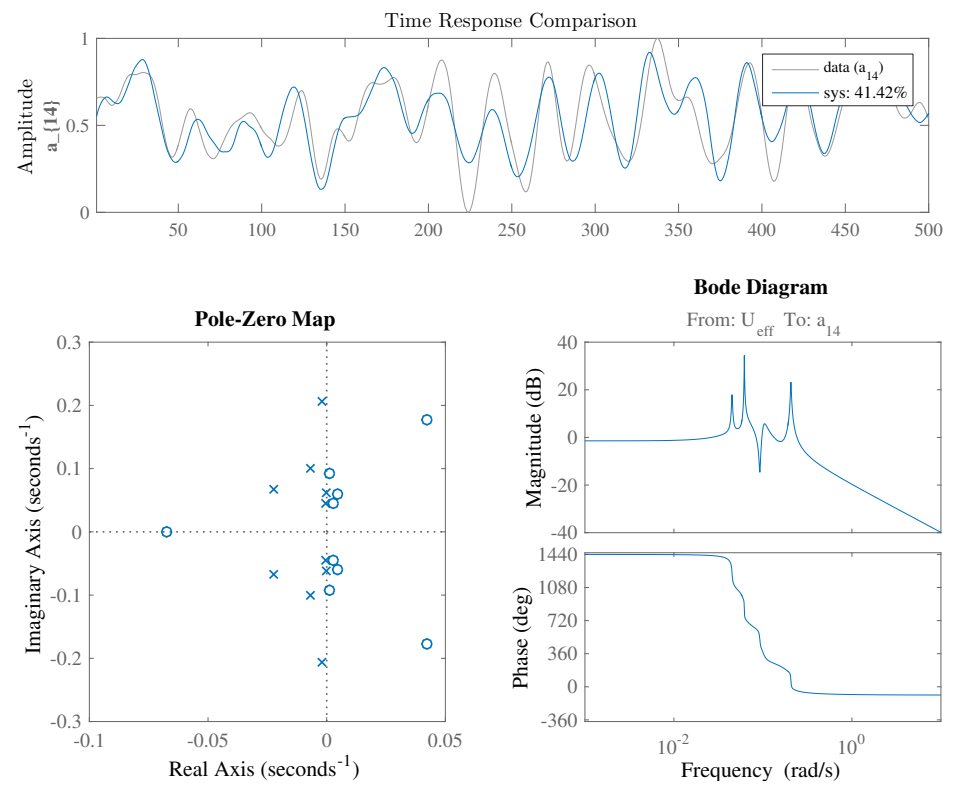

Figure E.0.14: Time response, pole-zero map, and Bode plot of the open-loop transfer function relating the effective inflow velocity $U_{\text {eff }}$ to the mode coefficient $a_{14}$. 